The role of strigolactones in resistance, tolerance and control of Striga infection in sorghum

Nasreldin Mohemed Ahmed 


\section{Thesis committee}

\section{Promotors}

Prof. dr Harro J. Bouwmeester

Professor of Plant Hormone Biology, Swammerdam Institute for Life Sciences, University of Amsterdam

Prof. dr Abdel Gabar Eltayeb Babiker

Enviroment, natural resources and desertification Research Institute. The National Research Center, Khartoum, Sudan.

\section{Other members}

Prof. dr ir PC Struik - Centre for Crop Systems Analysis, Wageningen University Prof. dr S Al-Babili - King Abdullah University of Science and Technology, Thuwal, Saudi Arabia

Dr ir L Bastiaans - Centre for Crop Systems Analysis, Wageningen University Dr W Kohlen - Laboratory of Molecular Biology, Wageningen University

This research was conducted under the auspices of the Graduate School of Experimental Plant Sciences (EPS) 


\title{
The role of strigolactones in resistance, tolerance and control of Striga infection in Sorghum
}

\section{Nasreldin Mohemed Ahmed}

\author{
Thesis \\ submitted in fulfillment of the requirements for the degree of doctor \\ at Wageningen University \\ by the authority of the Rector Magnificus, Prof. Dr. A.P.J. Mol, \\ in the presence of the
}

Thesis Committee appointed by the Academic Board to be defended in public on Friday 6 April 2018 at 11 a.m. in the Aula. 
Nasreldin Mohemed Ahmed

The role of strigolactones in resistance, tolerance and control of Striga infection in Sorghum 231pages.

$\mathrm{PhD}$ thesis, Wageningen University, Wageningen, NL (2018) with references, with summary in English.

ISBN: 978-94-6343-840-7

DIO: https://doi.org/10.18174/443824 


\section{Table of contents}

\section{Chapter1}

General Introduction

\section{Chapter 2}

Genetic variation in Sorghum bicolor: strigolactones and their role in resistance against Striga hermonthica.

\section{Chapter 3}

Genetic variation in tolerance to Striga infection in sorghum: underlying processes and the relationship with strigolactones.

\section{Chapter 4}

The role of strigolactones in Striga hermonthica control by non-host intercrops in sorghum.

\section{Chapter 5}

Evaluation of field resistance to Striga hermonthica [Del.] Benth. in Sorghum bicolor [L.] Moench. The relationship with strigolactones.

\section{Chapter 6}

\section{General Discussion}

Summary

Acknowledgements 


\section{Chapter 1}

\section{General Introduction}

\section{Sorghum importance and utilization worldwide}

The livelihood of more than $80 \%$ of the population in many African countries depends on agricultural production. In these countries poverty and malnutrition are increasingly affecting large sectors of the population. Improving agricultural output is vital to reduce poverty and improve food security (Rosegrant et al., 2001). Sorghum [Sorghum bicolor (L) Monech.], a drought tolerant Poaceous crop, is an important food source (grain) in the dryer parts of the world. It originates in eastern Africa, however, currently it is widely grown in Africa, India, China, USA and Mexico (Surwenshi et al., 2010) and is considered a major staple food for more than 500 million people in over 90 countries, where it is cultivated mostly for its edible grains, particularly in the developing world (ICRISAT. 2011). Sorghum, apart from being more heat and drought tolerant than maize (Zea mays L.), can also withstand periodic water logging without much damage (Taylor, 2003). The world's top producers include Sudan, Nigeria, India, USA, Mexico, China and Argentina (FAOSTAT, 2015). In the USA and some parts of the developing world, where sorghum is used as animal feed, and as feedstock for biofuel and the fiber industry, farmers use improved hybrids and advanced technologies. Whereas in Africa and parts of Asia subsistence farmers who have minimal access to production inputs such as fertilizer(s), pesticides, hybrid seeds, good soil, water and improved credit facilities are the main producers. Globally, sorghum is not only used for food, feed and beverage, but also as building material and in industry for production of starch and alcohol (Bantilan, et al., 2004). Sorghum grains, typically, have protein levels of around $9 \%$ and high levels of iron and zinc thus enabling humans to survive famine and escape malnutrition and associated diseases (Dicko et al., 2006). Because of climate change and water scarcity the crop is crucially important for food security in Africa, because unlike maize and rice it is drought resistant and can withstand periods of high temperature (Taylor, 2003). Nevertheless, abiotic stresses viz., drought, low soil fertility, salinity, soil acidity, cold and heat and biotic stresses encompassing diseases, insects, weeds, and birds are major production constraints. 


\section{The parasitic weed Striga}

In sub-Saharan Africa the root-parasitic weeds of the genus Striga are among the most serious pests attacking the main cereals including sorghum, maize (Zea mays [L.]), rice (Oryza L.), pearl millet [Pennisetum glaucum (L.) R. Br.] and sugarcane (Saccharum officinarum L.) (Parker and Rich 1993) (Fig. 1). Striga species present the largest challenge to food security in the region affecting the livelihood of over 300 million people in 25 countries (Kroschel, 1999; Ransom, 2000; Babiker, 2007; Ejeta, 2007a). In Africa Striga hermonthica [Del.] Benth. and Striga asiatica (L.) are the most devastating and widely spread parasitic weed species. The prevailing conditions such as drought spells and soil nutrient depletion favor the rapid expansion and proliferation of these parasites particularly in marginal areas of Africa (Ejeta, 2007a). Socioeconomic factors such as increased population pressure, limited education, monocropping, sub-optimal cultural practices, no or limited use of inputs and inflexibility to adopt new technologies have worsened the Striga problem especially for small subsistence farmers (Babiker, 2002). By now, over 21 million ha of arable land in Africa are infested by the worst species of the witchweeds, Striga hermonthica (Striga) resulting in a loss of 4.1 million tons of grain per year (Mboob, 1986) (Fig. 1). Currently the Striga problem in many African countries is pandemic and seems to be getting worse (Ejeta, 2007a).

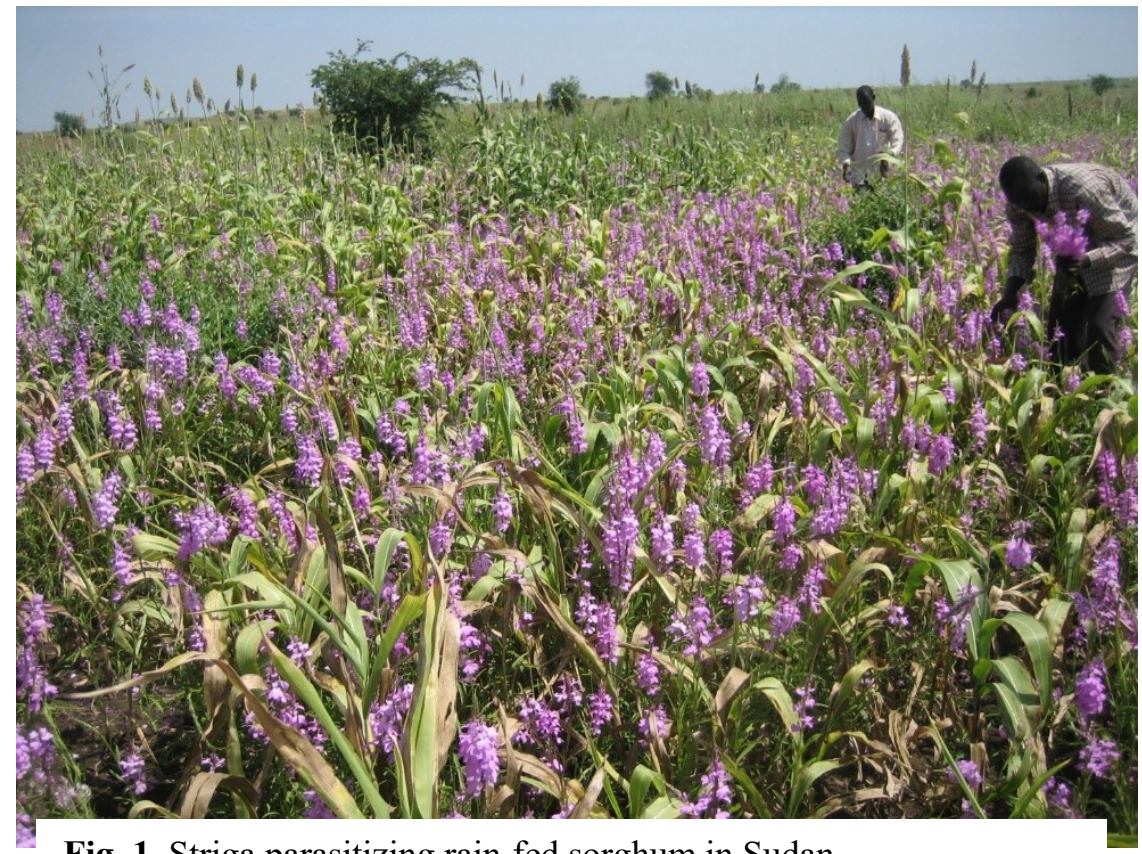

Fig. 1. Striga parasitizing rain-fed sorghum in Sudan. 


\section{The Striga life cycle}

The life cycle of Striga comprises a series of steps, such as stimulation of seed germination, initiation of an attachment organ (haustorium), penetration of the host root, connection to the host xylem and subsequent growth and development (Parker and Riches, 1993) (Fig. 2). To allow germination, Striga seeds need to loose dormancy under warm and moist conditions (a process called "pre-conditioning" in the lab) and subsequent exposure to specific exogenous signaling molecules exuded by the roots of their host (Bouwmeester et al., 2003). Roots of Striga hosts and some non-hosts may produce and release different germination stimulants (Sato et al., 2003). However, the strigolactones (strigolactones) are known to be the most potent stimulant, eliciting germination at nanomolar or even picomolar concentrations in seeds of many Striga species (Bouwmeester et al., 2003). Following germination, the Striga radicle grows (possibly chemotropically) towards the host root, where Striga stops growing upon perception of a second host-derived signal and forms a haustorium, the multicellular attachment and penetration organ (Fig. 2). The haustorium initiation represents the switch from the autotrophic to the parasitic mode of life (Babiker, 2007). The

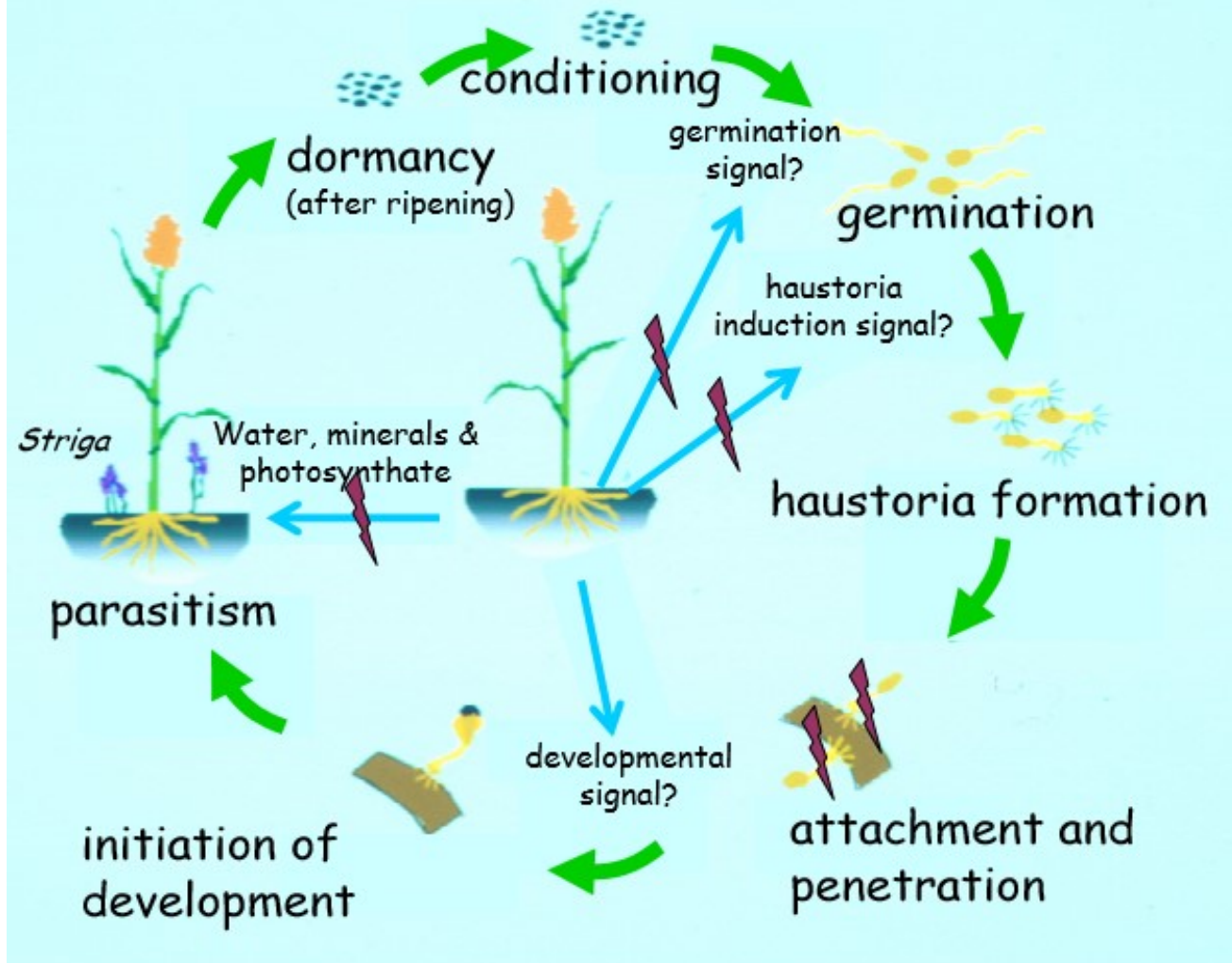

Fig. 2. Striga life cycle (from Ejeta et al., 1993). 
haustorium factor, 2,6-dimethoxy-p-benzoquinone (DMBQ), is a product of lignin oxidation and decarboxylation of phenolic acids found in plant cell walls described as one of the best exogenous haustorial inducing factors (Chang et al., 1986). After attachment the haustorium invades the host epidermal cells, penetrates into the root cortex and proceeds to form a xylem-to-xylem connection with the host and then starts to acquire the hosts nutrients and water. Subsequently adventitious roots are often produced, shoot development follows and Striga eventually emerges above ground and matures within a few weeks and sets seeds (Musselman, 1980) (Fig. 2).

\section{The impact of Striga on sorghum}

Heavy infestations by Striga often lead to complete crop failure (Ejeta et al. 1993; Hamdoun and Babiker 1988). Copious seed production and prolonged viability of the seeds result in a rapid buildup of huge seed banks in infested sorghum fields, which are eventually abandoned because the field becomes unsuitable for sorghum production (Ejeta et al. 1993; Hamdoun and Babiker 1988). Striga depends on its hosts for most of its needs viz., photo-assimilate, nitrogen assimilates, water, and amino acids (Pageau, et al., 2003; Cechin, 1993). High transpiration rates and permanent open stomata enable Striga to obtain most of its photo-assimilates from the host via the xylem-xylem connection (Musselman, 1980). Striga infected sorghum displays disease like symptoms including severe stunting, leaf chlorosis, necrosis and desiccation which lead ultimately to pre-mature wilting even under moist conditions (Stewart et al., 1991; Press et al., 1999). Such symptoms indicate a more complex interaction between the parasite and its host than a mere removal of nutrients (Berner et al., 1997). Striga infection on sorghum induces a remarkable reduction in photosynthesis (Gurney et al., 1995), which is considered to be the most important constraint to crop growth (Graves et al., 1989). The reduction in the rate of photosynthesis is predicted as a result of stomatal closure resulting from production and accumulation of abscisic acid (ABA) (Frost et al., 1997; Press et al., 1999). In addition, a decrease in stomatal conductance, transpiration rate, water use efficiency (WUE) and a decline in concentrations of growth regulators, cytokinin and giberellic acid (Gebremedhin et al., 2000; Drennan et al., 1979) were reported as consequence of infection. Recent studies indicated that Striga actively 
influences host transcription to foster parasitism by either up-regulating host genes associated with nutrient supply or by down-regulating defense-related genes (Spallek et al., 2013).

Crop germplasm may vary in their capacity to tolerate this negative impact of infection. Indeed, despite infection some germplasm displays less disease response to Striga parasitism and still produces a relatively acceptable yield (Van Ast, 2000). Breeding for both low infection level (resistance) and minimal Striga-induced host damage (tolerance) would be attractive but is a difficult challenge to conventional breeders (Kim, 1991; Kling, 1999). Tolerance to Striga is defined as the ability of genotypes to endure or minimize the consequences of infection (Kim et al., 1994; Rodenburg et al., 2006). It has been shown that Striga tolerant sorghum cultivars exhibit lower impairment of growth and grain yield compared with sensitive cultivars that are more strongly affected (poor growth and yield) despite a similar infection level (Rodenburg and Bastinaans, 2011; Rodenburg et al., 2005). The Sudanese sorghum cultivars Wad Ahmed and Mogud (Babiker, 2008; Wilson, 1953) and the Purdue lines P9405 and P9406, released in Tanzania under the names Hakaki and Wahi, respectively (Mbwaga et al, 2001) are examples of sorghum genotypes exhibiting noticeable field tolerance to Striga. However, it is quite difficult to distinguish resistance and tolerance and therefore also to select specifically for one of the two traits. For example, the sorghum genotypes CMDT39, Framida, IS9830 and SRN39 in many reports were described as tolerant and resistant (Rodenburg, 2005). However precise quantification of host tolerance (and resistance) is complicated by the non-linear relation between Striga infection level and yield loss (Gurney et al., 1999). Some studies have shown that changes in $\mathrm{CO}_{2}$-assimilation rates, $\mathrm{ABA}$ concentration, photosynthesis and chlorophyll fluorescence components were effected to a lesser extent by Striga infection in tolerant sorghum genotypes compared with sensitive ones and could thus be used as a marker for selection for tolerance (Rodenburg et al., 2008). The mechanisms that underlies tolerance to Striga is completely unknown. 


\section{Resistance mechanisms to Striga infection in sorghum}

The presence of genotypic differences in resistance to Striga in sorghum was reported already a long time ago (Doggett, 1965). Attempts to identify resistant varieties and to transfer resistance to high-yielding, well-adapted genotypes for many years have been a major component of many research programs with slow progress (Ejeta, 2007a). It is difficult and expensive to identify variations in resistance to Striga in the field among segregating populations. In addition, field resistance to Striga could be a result of actions and interactions of one or more resistance mechanisms, each of which might be controlled by different genes (Rao, 1983). However, specific bioassays have been developed to study resistance in individual stage of the Striga lifecycle such as the Agar gel, Extended Agar gel, Paper Roll and Sand Filled Plexi-Glass assay and a large collection of cultivated and wild sorghum germplasm has been screened using these assays to detect the presence of one or more specific resistance mechanisms and to identify resistance phenotypes that discourage parasitic growth and establishment at key stages of the parasite life cycle (Ejeta, 2005). Based on these assays sorghum germplasm with superior resistance levels was identified and resistance forms were characterized and separated into specific mechanisms including pre-attachment resistance associated with low germination stimulant production (LGS) and/or low haustorial initiation factor and inhibition of germ tube exoenzymes by root exudates (Haussmann et al., 2000a; Ejeta, 2007). Post-attachment resistance is expressed as delayed parasite development or fewer Striga attachments due to mechanical barriers (Amusan et al., 2011), resistance due to a hypersensitive responses characterized by necrosis of the tissue around the parasite attachment site which is assumed to prevent invasion of the host root cortex, an incompatible response, or antibiosis i.e. reduced Striga development through unfavorable phytohormone supply by the host (Mohamed et al., 2003; Ejeta, 2007c). Such different resistance mechanisms have been described in different sorghum sources, i.e. low production of the germination stimulant (SRN39, IS9830, Framida, 555, SAR lines, IS15401); low haustorial initiation (accession P-78 of Sorghum drummondii); mechanical barriers (N13, Framida); antibiosis (SRN39, N13); and hypersensitivity (SAR 16, SAR 19, SAR 33, Sorghum versicolor) (Hausmann et al., 
2000). However, more information is needed about individual resistance mechanisms in different sources of resistance so that they can be pyramided in suitable genotypes.

\section{Is germination a target for Striga control?}

As described above, Striga seeds germinate only in response to germination stimulants exuded by the host plant (Bouwmeester et al., 2003). Decades ago already, during 19751980, at the International Crops Research Institute for the Semi-Arid Tropics (ICRISAT), 15000 sorghum genotypes were screened for their capacity to stimulate germination of $S$. asiatica seeds. Of these genotypes $10 \%$ were identified as low stimulant producers (Vasudeva Rao, 1985). Subsequent field screening showed that the proportion of field resistance among the low stimulant producing genotypes is higher than among the high stimulant producing genotypes (Rao, 1983). This association of low stimulant production with field resistance was later also confirmed by Hess et al., (1992) and the genotypes SRN39, IS9830 IS and 87441 (Framida), which were low stimulants producers, were identified as resistant (Hess et al., 1992). Indeed, resistance based on low germination stimulation is the first and most exploited resistance mechanism against Striga. In 1991 line SRN39 and IS9830 with resistance linked to low germination-stimulating activity were released for cultivation in Striga endemic areas of the Sudan (Parker and Riches, 1993). But efforts to unravel the genes that underlie the low germination stimulation resistance mechanism started only relatively recently (Kapran et al., 2007). Laboratory analysis of a population developed from crosses between SRN39 and the higher stimulant genotype Shanqui Red using the agar gel germination assay (Vogler et al., 1996) discovered a single recessively inherited QTL, lgs (low germination stimulant), which accounted for the low stimulation resistance mechanism (Satish et al., 2012). Using the sorghum genome sequence available from the Phytozome database lgs was fine-mapped to the short arm of the sorghum chromosome SBI-05 at a $5.8 \mathrm{cM}$ interval closest to three microsatellite markers, (SSR) SB3344, SB3346 and SB3343 with approximately $400 \mathrm{~kb}$ physical sequence distance. The SRN39 alleles at the lgs locus were found to be shared for the tightly linked (SSR) markers by other Striga resistant genotypes e.g. Tetron and IS9830 suggesting lgs is common among these genotypes with low Striga germination stimulant 
activity (Satish et al., 2012). Fine mapping recently identified

\section{Box 1: Breeding for Striga resistance in Sudan}

Striga research in Sudan has long been part of an internationally coordinated network involving the International Crops Research Institute for the Semi-Arid Tropics, International Development Research Center, International Sorghum and Millet (ICRISAT, IDRC, INTSORMIL) (Hamdoun, 1988; Babiker, 2008). The goal was to assist national research programs in African countries to develop sorghum cultivars with durable resistance to Striga and deploy them as an integral component of an integrated management strategy for the parasite (Babiker, 2008). However, development of Striga resistant cultivars has been slow due to the limited knowledge of the genetics of Striga resistances and the difficulty in evaluating resistance in the field (Haussmann et al., 2000b). Nevertheless, a number of Quantitative Trait Loci (QTLs) were identified in the resistant donor N13 that are associated with post attachment resistance (Maiti et al., 1984) and the molecular markers flanking these QTLs published (Haussmann et al., 2004) providing an opportunity to transfer the QTLs from the parental line N13 into susceptible genotypes with preferred agronomic qualities through Marker Assisted Selection (MAS) (Gamar et al., 2007). Very recently this has resulted in release of the first products of MAS for Striga resistant lines (T1BC3S4, AG6BC3S4, AG2BC3S4 and $\mathrm{W} 2 \mathrm{BC} 3 \mathrm{~S} 4)$ combining post attachment Striga resistance and farmer preferences for cultivation in Sudan (Mohamed et al., 2014). Application of marker technology should now also enable transfer of other alleles encoding different resistance mechanisms viz., lgs and other post-attachment resistance QTLs thus improving resistance durability and adoption rates (Haussmann et al., 2000a). Although, growing Striga resistant cultivars may offer a long-lasting control means, yet strategies based on integrating use of resistant and/or tolerant cultivars, agronomic practices, chemical and other control options are, always, anticipated to be more sustainable .

the underlying gene, a sulfotransferase with as yet unknown function (Gobena et al., 2017).

\section{Strigolactones}


Germination of Striga is induced by strigolactones (Xie et al., 2010). They are a recently discovered family of plant hormones which help plants to communicate with their environment (Andreo et al., 2015). The strigolactones seem to be synthesized mainly in the roots and have diverse roles in plant development (Al-Babili and Bouwmeester, 2015). Progresses in the isolation and analysis using ultra-performance liquid chromatography coupled to tandem quadrupole mass spectrometry (UPLC-MS/MS) have improved characterization and quantification of strigolactones in many plant species (Sato et al., 2005). The first strigolactone, strigol was isolated from the root exudate of cotton plants (Gossypium hirsutum L.) a non-host species (Cook et al., 1966). Orobanchol and alectrol were isolated from cowpea and red clover; strigol, sorgomol and 5-deoxystrigol were detected in maize, millet and sorghum (Hauck et al., 1992; Siame et al., 1993; Awad et al., 2006; Xie et al., 2008). The universal occurrence of strigolactones is possibly due to their role in stimulating hyphal branching of arbuscular mycorrhizal (AM) fungi. The symbiotic fungus, on colonization of the host roots, increases nutrients and water uptake (Nadal et al., 2013). In addition, strigolactones regulate plant responses to abiotic factors including nutrient availability and light. For example, under low-phosphate conditions, they reduce the shoot to root ratio, through inhibition of shoot branching and increase in lateral root formation which assists plants to exploit the limited nutrients in the soil (Cardoso et al., 2011).

Amounts and composition of strigolactones may vary among different plant species as well as among different cultivars of the same species (Xie et al., 2010). The basic structural unit of strigolactones is a tricyclic lactone (A, B, C-rings) which connects via an enol ether bridge to a butyrolactone (D-ring) which is necessary for biological activity. According to the stereochemistry of the C-ring, strigolactones, can be organized into two groups with $\alpha$-orientation (orobanchol-like) or $\beta$-orientation (strigollike) (Zwanenburg et al., 2013; Xie et al., 2013). Striga seed germination was found to be more sensitive to strigol-like group (Nomura et al., 2013; Sugimoto et al., 1998). While AM fungi hyphal branching was found to be sensitive to both orobanchol- and strigol-like strigolactones, they are more sensitive to certain types of AB-ring decorations (Akiyama et al., 2010). Lopez-Raez et al. (2008) indicated that strigolactone biosynthesis in tomato is strongly induced by phosphate starvation. 
Yoneyama et al., (2007) showed that strigolactone production and secretion from roots tissues of sorghum increases significantly under low $\mathrm{P}$ and $\mathrm{N}$ conditions which might explain the wide spread and higher Striga infection of crops grown under low nutrient condition particularly in sub-Saharan Africa.

\section{Strigolactones of sorghum}

Strigolactones produced by sorghum include strigol, sorgolactone, sorgomol and 5deoxy-strigol that have been characterized individually and/or in a different combination in some cultivars (Siame et al., 1993; Hauck et al., 1992; Xie et al., 2008; Awad et al., 2006). Individual compounds or strigolactone blends produced may differ in activity to stimulate Striga seed germination. Cardoso et al. (2011) showed that sorgomol and ent-2'-epi-orobanchol induced higher Striga seed germination than (-)orobanchol which hardly induced any germination. As described above, many reports show that low Striga germination stimulant activity in sorghum is associated with field resistance to Striga (Ramaiah 1987; Hess et al., 1992; Hess et al., 1992; Haussmann et al., 2004). Recent work by Yoneyama et al. (2010) showed that the Striga-susceptible sorghum cultivar Tabat exuded high amounts of 5-deoxystrigol while the Strigaresistant cultivar SRN39 exuded low amounts of 5-deoxystrigol. Recently, Gobena, et al., (2017) showed that expression of the $L G S$ sulfotransferase in SRN39 is absent and associated with loss of function which results in the production of a high orobanchol/low 5-deoxystrigol profile which stimulates less Striga seed germination. In contrast, the sulfotransferase is expressed in the susceptible cultivar Shanqui Red, which results in production of a high 5-deoxystrigol/low orobanchol strigolactone profile which is associated with high germination inducing activity and high field Striga infection. The exact catalytic function of this sulfotransferase still needs to be resolved. This lack of understanding shows that manipulation of germination-stimulating activity to acquire low stimulation resistance mechanism requires sufficient knowledge on strigolactone biosynthesis (Scholes et al., 2008). Previously, screening of maize mutants (Matusova et al., 2005) showed that strigolactones are derived from the carotenoid pathway. It was shown lately that strigolactone biosynthesis starts with isomerization of the carotenoid compound all-trans- $\beta$-carotene to 9-cis- $\beta$-carotene 
through activity of the carotenoid isomerase enzyme identified in rice as DWARF27 (D27) (Alder et al., 2012). Subsequently, 9-cis- $\beta$-carotene is cleaved in two consecutive steps by two carotenoid cleavage dioxygenases, first by CCD7 (D17 or HTD1 in rice) producing 9-cis- $\beta$-apo-10'-carotenal, followed by cleavage and molecular rearrangement performed by $C C D 8$ (D10 in rice) leading to carlactone (Alder et al., 2012). Zhang et al. (2014) showed that the cytochrome P450s identified in Arabidopsis as $M A X 1$ and in rice as MAX1 homologs (Os01g0700900) convert carlactone into the parent strigolactone, ent-2'-epi-5-deoxystrigol (4-deoxyorobanchol). Moreover, in rice a second MAX1 homolog (Os01g0701400) catalyzes hydroxylation of ent-2'-epi-5deoxystrigol to orobanchol thus identifying enzymes involved in strigolactone biosynthesis and subsequent structural diversification (Zhang et al., 2014). Characterization of further enzymes involved in the structural diversification of the strigolactones should provide the tools to adapt the strigolactone composition in Striga host plants (e.g. sorghum) towards types with less stimulation activity, such as for example orobanchol.

\section{Striga control}

Successful Striga control has been difficult to achieve through conventional cultural, chemical and biological methods such as fallowing, hand-pulling, fertilization and time of planting (Parker et al., 1993; Ransom, 1999; Koyama, 2000; Oswald, 2005; Kgosi et al., 2012). Some successes have been obtained with the use of herbicides (Last, 1960, 1961; Babiker et al., 1996) more recently also in combination with herbicide resistant or tolerant crops (Tuinstra, 2009; Kabambe et al., 2008). A number of control methods use germination of Striga as their target. This holds for the approach of suicidal germination in which synthetic strigolactone analogs are used to induce stimulation of seed germination in the absence of a host. Also the use of intercropping seems to be based on this principle. Many of the crops that have been investigated for this purpose were shown to induce high levels of Striga germination. As they are, however, not a host for Striga this results in suicidal germination. 


\section{Box 2: The future of Striga research}

In the era of genomics (e.g. Parasitic Plant Genome Project) scientists are investigating genes responsible for Striga parasitism which might help in engineering host-derived resistance by transformation of host crops with RNAi sequences targeting critical Striga genes. New approaches to identify chemicals and crops with altered strigolactone levels that stimulate more beneficial mycorrhizal association and reduce Striga germination are also being explored. Such studies include investigations on the sensitivity of parasitic plants to strigolactones and correlations between strigolactone structure and the Striga strigolactone receptors. For example Toh et al., (2015) showed that Striga sensitivity to strigolactones is influenced by Striga receptor sensitivity and host derived strigolactone structure and stereochemistry. The authors found that seeds of transgenic Arabidopsis expressing the Striga ShHTL7 strigolactone receptor are more sensitive to 5-deoxystrigol and 4-deoxyorobanchol than to strigol. The author proposed that the presence of a hydroxyl group on carbon 5 of the A-ring of strigol decreases binding to this receptor and that this may explain why crop cultivars producing high levels of 5-deoxystrigol that lacks this hydroxyl group are so susceptible to infection.

\section{This thesis}

The parasitic weed Striga has a huge impact on sorghum and we are in dire need for new possibilities for control. In Chapter 1 I introduce the Striga problem and describe what has so far been done to break the spell. For example, through the use of resistant varieties, crop rotation and intercropping. I particularly focus on the first step in the life cycle of Striga: germination, as a target for resistance breeding and control methods. Although there is increasing insight in the importance of strigolactones for Striga germination stimulant activity, the knowledge about how this translates to the field is very limited. Therefore, in Chapter 2 I analyse the strigolactones in and germination stimulant activity of root exudates of a collection of sorghum genotypes, mostly from 
Sudan. I also analyse how the strigolactone composition affects the germination stimulant activity of the exudates of these genotypes and, finally, how this translates to infection in a pot experiment. In Chapter 3 I study the genetic variation in tolerance to Striga in sorghum. I do that by analysing the effect of Striga on morphological and physiological traits including plant height, biomass accumulation, leaf area, photosynthesis, stomatal conductance and ABA. In addition, I investigate whether there is a link between strigolactones and tolerance. In Chapter 4 I investigate the role of strigolactones in the efficiency of intercrops sesame and groundnut to control Striga in sorghum and of millet as a rotation crop. I report the identification of a number of strigolactones in groundnut and sesame that have not been analysed before. In Chapter 5 I perform the proof of concept for much of what I studied in my thesis by presenting results of a field experiment with natural Striga infestation in two rain-fed fields in eastern Sudan to evaluate how the different levels of strigolactone production in a selection of sorghum genotypes affect field infection by the parasite. In Chapter 6 I discuss the most important results from my thesis and reflect on future perspectives of Striga research. 


\section{References}

Akiyama, K., Ogasawara, S., Ito, S., Hayashi, H., 2010. Structural requirements of strigolactones for hyphal branching in AM fungi. Plant and cell physiology 51, 1104-1117.

Al-Babili, S., Bouwmeester, H.J., 2015. Strigolactones, a Novel Carotenoid-Derived Plant Hormone. Annual review of plant biology 66, 161-186.

Alder, A., Jamil, M., Marzorati, M., Bruno, M., Vermathen, M., Bigler, P., Ghisla, S., Bouwmeester, H., Beyer, P., Al-Babili, S., 2012. The path from $\beta$-carotene to carlactone, a strigolactone-like plant hormone. Science 335, 1348-1351.

Amusan, I.O., Rich, P.J., Housley, T., Ejeta, G., 2011. An In Vitro Method for Identifying Postattachment Resistance in Maize and Sorghum. Agronomy Journal 103, 1472-1478.

Andreo-Jimenez, B., Ruyter-Spira, C., Bouwmeester, H.J., Lopez-Raez, J.A., 2015. Ecological relevance of strigolactones in nutrient uptake and other abiotic stresses, and in plant-microbe interactions belowground. Plant and Soil 394, 1-19.

Awad, A.A., Sato, D., Kusumoto, D., Kamioka, H., Takeuchi, Y., Yoneyama, K., 2006. Characterization of strigolactones, germination stimulants for the root parasitic plants Striga and Orobanche, produced by maize, millet and sorghum. Plant Growth Regulation 48, 221-227.

Babiker, A. G. T. (2007). Striga: The spreading scourge in Africa. Regulation of Plant Growth and Development. 43.1: 74-87.

Babiker, A. G.T. Butler, L. and Ejeta, G., (1996). Integrated use of Striga resistant sorghum varieties with cultural and chemical control. In: The International Conference on Genetic Improvement of sorghum and Pearl Millet. Taxas A and M University Research and Extension Centre, Luubbock, USA, pp 517-524.

Babiker, AGT (2002). Striga control in Sudan: An integrated approach. In, Leslie, J F(ed). Sorghum and Millet Diseases, pp 159-163. Iowa State Press.

Babiker, A. (2008). Striga control in Sudan: An integrated approach. In: Leslie, J.F. (edit) Sorghum and Millets Diseases, Iowa State Press. Pp 159-163.

Bantilan, C.S., Gowda, C.L.L., Reddy, B.V.S., Obilana, A.B., Evenson, R.E., 2004. Sorghum genetic enhancement: research process, dissemination and impacts. International Crops Research Institute for the Semi-Arid Tropics.

Ransom, J.K., 2000. Long term approaches for the control of Striga in cereals: field management. Crop Prot $19,759-763$.

Rodenburg J, Bastinaans L. 2011. Host-plant defence against Striga spp.:reconsidering the role of tolerance. Weed Research 51, :438-441

Rodenburg J, Bastinaans L, Weltzien E, and Hess DE. 2005. How can field selection for Striga resistance and tolerance in sorghum be improved? Field Crops Research. 93, 34-50.

Berner, D.K., Winslow, M.D., Awad, A.E., Cardwell, K.F., Raj, D.R.M., Kim, S.K., 1997. Striga research methods. Manual, The Pan-African Striga Control Network (PASCON) and the International Institute of Tropical Agriculture, 13-20.

Bouwmeester, H.J., Matusova, R., Zhongkui, S., Beale, M.H., 2003. Secondary metabolite signalling in host-parasitic plant interactions. Curr. Opin. Plant Biol. 6, 358-364.

Cardoso, C., Ruyter-Spira, C., Bouwmeester, H.J., 2011. Strigolactones and root infestation by plantparasitic Striga, Orobanche and Phelipanche spp. Plant Science 180, 414-420.

Cechin, I., Press, M.C., 1993. Nitrogen relations of the sorghum-Striga hermonthica hostparasite association: growth and photosynthesis. Plant, Cell \& Environment 16, 237-247.

Chang, M., Lynn, D.G., 1986. The haustorium and the chemistry of host recognition in parasitic angiosperms. Journal of Chemical Ecology 12, 561-579.

Cook, C., Whichard, L.P., Turner, B., Wall, M.E., Egley, G.H., 1966. Germination of witchweed (Striga lutea Lour.): isolation and properties of a potent stimulant. Science 154, 1189-1190. 
Drennan, D. S. H., \& El Hiweris, S. O. (1979). Changes in growth regulating substances in Sorghum vulgare infected by Striga hermonthica. In Proceedings Second International Symposium on Parasitic Weeds. Eds LJ Musselman, AD Worsham, RE Eplee. University Graphics, North Carolina State University, Raleigh pp. 144-155

Dicko, M.H., Gruppen, H., Traore, A.S., Voragen, A.G.J., Van Berkel, W.J.H., 2006. Review: sorghum grain as human food in Africa: relevance of starch content and amylase activities. African journal of biotechnology 5, 384-395.

Doggett, H., 1965. Striga hermonthica on sorghum in East Africa. The Journal of Agricultural Science 65, 183-194.

Doggett, H., 1988. Sorghum. Longman Scientific and Technical.

Drennan, D.S.H., El Hiweris, S.O., Changes in growth regulating substances in Sorghum vulgare infected by Striga hermonthica. pp. 144-155.

Ejeta, G., 2005. Integrating biotechnology, breeding, and agronomy in the control of the parasitic weed Striga spp in sorghum. In the Wake of the Double Helix: From the Green Revolution to the Gene Revolution. Tuberosa R, Phillips RL, Gale M (Eds.). Bologna, Italy, 239-251.

Ejeta, G., L. G. Butler, and A.G.T. Babiker. (1993). New Approaches to the Control of Striga; Striga Research at Purdue University. West Lafayette, IN: Purdue University Agricultural Experiment Station research bulletin $991.27 \mathrm{p}$.

Ejeta, G. (2007a). The Striga scourge in Africa: a growing pandemic. In: Ejeta G. and Gressel J. (eds). Integrating New Technologies for Striga Control: Towards ending the witch-hunt. World Scientific Publishing Co. Pte Ltd, 5 Tol Tuck Link, Singapore, 3-16.

Ejeta, G., 2007 b. Breeding for Resistance in Sorghum: Exploitation of an Intricate Host-Parasite Biology. Crop Science 47, S-216-S-227.

Ejeta, G., Butler, L.G., 1993. Host-parasite interactions throughout the Striga life cycle, and their contributions to Striga resistance. African Crop Science Journal 1.

Ejeta, G., Rich, P.J., Mohamed, A., 2007c. Dissecting a complex trait to simpler components for effective breeding of sorghum with a high level of Striga resistance. Integrating new technologies for Striga control—-towards ending the witch hunt. World Scientific, Singapore, 87-98.

FAOSTAT, 2015. Food and Agriculture Organization Of the United Nations, statistic division. fao.org, http://faostat.fao.org/.

Frost, D.L., Gurney, A.L., Press, M.C., Scholes, J.D., 1997. Striga hermonthica reduces photosynthesis in sorghum: the importance of stomatal limitations and a potential role for ABA? Plant, Cell \& Environment 20, 483-492.

Gamar, Y., 2007. Deployment of marker assisted selection, for introgressing Striga resistance in sorghum, Greener Journal of Agricutral Science., 3(7), 550-556.

Gebremedhin, W., Goudriaan, J., Naber, H., 2000. Morphological, phenological and water-use dynamics of sorghum varieties (Sorghum bicolor) under Striga hermonthica infestation. Crop Protect. 19, 61-68.

Gobena, D., Shimels, M., Rich, P. J., Ruyter-Spira, C., Bouwmeester, H., Kanuganti, S., and Ejeta, G. (2017). Mutation in sorghum LOW GERMINATION STIMULANT 1 alters strigolactones and causes Striga resistance. Proceedings of the National Academy of Sciences, 114(17), 4471-4476.

Graves, J.D., Press, M.C., Stewart, G.R., 1989. A carbon balance model of the sorghum-Striga hermonthica host-parasite association. Plant, Cell \& Environment 12, 101-107.

Gurney, A.L., Press, M.C., and Ransom, J.K. (1995). The parasitic angiosperm Striga hermonthica can reduce photosynthesis of its sorghum and maize hosts in the field. Journal of Experimental Botany 46(12), 1817-1823.

Gurney, A., Press, M., Scholes, J., 1999. Infection time and density influence the response of sorghum to the parasitic angiosperm Striga hermonthica. New Phytologist 143, 573-580.

Hamdoun, A.M., Babiker, A.G.T., 1988. Striga in the Sudan: research and control. Proceedings of the Food and Agriculture Organization/Organization of African Unity All African Government Consultation on Striga Control, Maroua, Cameroon, 90-91. 
Hauck, C., Müller, S., Schildknecht, H., 1992. A germination stimulant for parasitic flowering plants from Sorghum bicolor, a genuine host plant. J. Plant Physiol. 139, 474-478.

Haussmann, B., Hess, D., Reddy, B., Mukuru, S., Kayentao, M., Welz, H., Geiger, H., 2001. Quantitativegenetic parameters of sorghum growth under Striga infestation in Mali and Kenya. Plant Breeding $120,49-56$.

Haussmann, B.I., Hess, D.E., Omanya, G.O., Folkertsma, R.T., Reddy, B.V., Kayentao, M., Welz, H.G., Geiger, H.H., 2004. Genomic regions influencing resistance to the parasitic weed Striga hermonthica in two recombinant inbred populations of sorghum. TAG. Theoretical and applied genetics. Theoretische und angewandte Genetik 109, 1005-1016.

Haussmann, B.I., Hess, D.E., Welz, H.-G., Geiger, H.H., 2000a. Improved methodologies for breeding Striga-resistant sorghums. Field Crops Research 66, 195-211.

Haussmann, B.I.G., Hess, D.E., Reddy, B.V.S., Welz, H.G., Geiger, H.H., 2000b. Analysis of resistance to Striga hermonthica in diallel crosses of sorghum. Euphytica 116, 33-40.

Hess, D.E., Ejeta, G., Butler, L.G., 1992. Selecting sorghum genotypes expressing a quantitative biosynthetic trait that confers resistance to Striga. Phytochemistry 31, 493-497.

Kabambe, V. H., Kanampiu, F., \& Ngwira, A. (2008). Imazapyr (herbicide) seed dressing increases yield, suppresses Striga asiatica and has seed depletion role in maize (Zea mays L.) in Malawi. African Journal of Biotechnology, 7(18).

Kapran, I., Grenier, C., Ejeta, G., 2007. Introgression of genes for Striga resistance into African landraces of sorghum. Integrating New Technologies for Striga Control: Towards Ending the Witch-Hunt, pp. 129-141.

Kim, S., 1991. Breeding for tolerance and general resistance in Maize: a novel approach to combating Striga in Africa. Nairobi, Kenya, 168-176.

Kim, S.K., Akintunde, A.Y., Walker, P., 1994. Responses of maize, sorghum and millet host plants to infestation by Striga hermonthica. Crop Protection 13, 582-590.

Kling, J., Fajemisin, J., Badu-Apraku, B., Diallo, A., Menkir, A., Melake-Berhan, A., 1999. Striga resistance breeding in maize. Breeding for Striga Resistance in Cereals. Proceedings of a Workshop; Ibadan, Nigeria; 18-20 Aug 1999.

Koyama, M. L. (2000). Molecular markers for studying pathogen variability: Implications for breeding for resistance to Striga hermonthica. InBreeding for Striga Resistance in Cereals, (Eds B. I. G. Haussmann, D. E. Hess, M. L. Koyama, L. Grivet, H. F. W. Rattunde, and H. H. Geiger). Weikersheim, Germany: Margraf Verlag, pp 227-245.

Kroschel, J. (1999). Analysis of the Striga problem, the first step towards future joint action. In Advances in Parasitic Weed Control at On-farm Level. Vol. I. Joint Action to Control Striga in Africa, (Eds J. Kroschel, H. Mercher-Quarshie, and J. Sauerborn,) J. Weikersheim, Germany: Margraf Verlag, pp $3-25$.

Last, F. T. (1960). Effect of cultural treatments on the incidence of Striga hermonthica (Del.) Benth. and yields of Sorghum in the Sudan: field experiments 1957/8. Annals of Applied Biology, 48(2), 207229.

Last, F. T. (1961). Direct and residual effects of Striga control treatments on sorghum yields. Tropical Agriculture, Trinidad, 38, 49-55.

Lopez-Raez, J.A., Charnikhova, T., Gomez-Roldan, V., Matusova, R., Kohlen, W., De Vos, R., Verstappen, F., Puech-Pages, V., Becard, G., Mulder, P., 2008. Tomato strigolactones are derived from carotenoids and their biosynthesis is promoted by phosphate starvation. New Phytologist 178, 863874.

Maiti, R.K., Ramaiah, K.V., Bisen, S.S., Chidley, V.L., 1984. A comparative study of the haustorial development of Striga asiatica (L.) Kuntze on sorghum cultivars. Annals of botany 54, 447-457.

Matusova, R., Rani, K., Verstappen, F.W.A., Franssen, M.C.R., Beale, M.H., Bouwmeester, H.J., 2005. The strigolactone germination stimulants of the plant-parasitic Striga and Orobanche spp. are derived from the carotenoid pathway. Plant physiology 139, 920-934. 
Mboob, S.S., 1986. A regional program for Striga control in West Africa. Improved Striga Management for Africa. Proc. FAO/OAU All Africa Govt. Consultation on Striga Control, Accra, Ghana. FAO, Rome, Italy, 190-194.

Mbwaga et al., R.C., Massawe C, Lamboll RI. 2001., 2001. Evaluation of Sorghum lines for Striga resistance and their performance on farmer fields in Tanzania. . 7th International Parasitic Weed Symposium, Nantes, France, Université de Nantes.

Mohamed, A., Ali, R., Elhassan, O., Suliman, E., Mugoya, C., Masiga, C. W., ... \& Hash, C. T. (2014). First products of DNA marker-assisted selection in sorghum released for cultivation by farmers in subsaharan Africa. Journal of Plant Science \& Molecular Breeding, 3(3), 1-10.

Mohamed, A., Ellicott, A., Housley, T.L., Ejeta, G., 2003. Hypersensitive Response to Infection in. Crop science 43, 1320-1324

Musselman, L. J. (1980). The biology of Striga, Orobanche, and other root-parasitic weeds. Annual review of phytopathology, 18(1), 463-489.

Nadal, M. and Paszkowski, U. (2013). Polyphony in the rhizosphere: presymbiotic communication in arbuscular mycorrhizal symbiosis. Current Opinion in Plant Biology 16: 473-479.

Network, A. B., Doggett, H., Ayensu, E. S., Keynes, R. D., Marton-Lefevre, J., \& Musselman, L. J. (1984). Striga: biology and control pp. 179.

Nomura, S., Nakashima, H., Mizutani, M., Takikawa, H., Sugimoto, Y., 2013. Structural requirements of strigolactones for germination induction and inhibition of Striga gesnerioides seeds. Plant cell reports, pp 32, 829-838.

Oswald, A. Striga control—technologies and their dissemination (2005). Crop Protection 24.4: 333-342.

Pageau, K., Simier, P., Le Bizec, B., Robins, R.J., Fer, A., 2003. Characterization of nitrogen relationships between Sorghum bicolor and the root-hemiparasitic angiosperm Striga hermonthica (Del.) Benth. using K15NO3 as isotopic tracer. Journal of Experimental Botany 54, 789-799.

Parker, C., Riches, C.R., 1993. Parasitic weeds of the world: biology and control. CAB international pp.332.

Press, M.C., Scholes, J.D., Watling, J.R., 1999. Parasitic plants: physiological and ecological interactions with their hosts. Physiological plant ecology, pp 175-197.

Ramaiah, K. V. (1987). Control of Striga and Orobanche species-a review. In Proceedings of the 4th international symposium on parasitic flowering plants. pp. 637-664.

Rao, M.J.V., 1983. Patterns of resistance to Striga asiatica in sorghum and millets, with special reference to Asia, pp 96-112.

Ransom, J. K. (1999). The status quo of Striga control: Cultural, chemical and integrated aspects. In Advances in Parasitic Weed Control at On-farm Level. Vol. I. Joint Action to Control Striga in Africa, 133-143. (Eds J. Kroschel, H. Mercher-Quarshie and J. Sauerborn). Weikersheim, Germany: Margraf Verlag

Riopel, J.L., Timko, M.P., 1995. Haustorial initiation and differentiation. Parasitic plants, 39-79.

Rodenburg, J. (2005) The role of sorghum genotype in the interaction with the parasitic weed Striga hermonthica. $\mathrm{PhD}$ thesis, Wageningen University, Wageningen, The Netherlands.

Rodenburg, J., Bastiaans, L., Kropff, M.J., 2006. Characterization of host tolerance to Striga hermonthica. Euphytica 147, 353-365.

Rodenburg, J., Bastiaans, L., Schapendonk, A.H., van der Putten, P.E., van Ast, A., Dingemanse, N.J., Haussmann, B.I., 2008. CO2-assimilation and chlorophyll fluorescence as indirect selection criteria for host tolerance against Striga. Euphytica 160, 75-87.

Rosegrant, M.W., Meijer, M.S. and S Witcover, J., 2001. 2020 global food outlook trends, alternatives, and choise. International Food Policy Research Institute, Washington, DC (EUA) No. 338.19 T974tw.

Satish, K., Gutema, Z., Grenier, C., Rich, P.J., Ejeta, G., 2012. Molecular tagging and validation of microsatellite markers linked to the low germination stimulant gene (lgs) for Striga resistance in sorghum [Sorghum bicolor (L.) Moench]. TAG. Theoretical and applied genetics. Theoretische und angewandte Genetik 124, 989-1003. 
Sato, D., Awad, A.A., Chae, S.H., Yokota, T., Sugimoto, Y., Takeuchi, Y., Yoneyama, K., 2003. Analysis of strigolactones, germination stimulants for Striga and Orobanche, by high-performance liquid chromatography/tandem mass spectrometry. Journal of agricultural and food chemistry 51, $1162-$ 1168 .

Sato, D., Awad, A. A., Takeuchi, Y., \& Yoneyama, K. (2005). Confirmation and quantification of strigolactones, germination stimulants for root parasitic plants Striga and Orobanche, produced by cotton. Bioscience, biotechnology, and biochemistry, 69(1), 98-102.

Scholes, J.D., Press, M.C., 2008. Striga infestation of cereal crops-an unsolved problem in resource limited agriculture. Current opinion in plant biology 11, 180-186.

Shinde, V. K., and N. Kulkarni. "Genetics of resistance to Striga asiatica in sorghum." In Proceedings of the ICRISAT-ICAR Working Group Meeting on Striga Control, vol. 30, pp. 134-141. 1982.

Siame, B.A., Weerasuriya, Y., Wood, K., Ejeta, G., Butler, L.G., 1993. Isolation of strigol, a germination stimulant for Striga asiatica, from host plants. Journal of Agricultural and Food Chemistry 41, 14861491.

Spallek, T., Mutuku, M., Shirasu, K., 2013. The genus Striga: a witch profile. Molecular plant pathology 14, 861-869.

Stewart, G.R., Press, M.C., Graves, J.D., Nour, J.J., and Wylde, A. (1991). A physiological characterization of the host parasite association between Sorghum bicolor and Striga hermonthica and its implication for Striga control. combating Striga in Africa. Kim SK (Ed) pages, 45-54.

Sugimoto, Y., Wigchert, S.C.M., Thuring, J.W.J.F., Zwanenburg, B., 1998. Synthesis of all eight stereoisomers of the germination stimulant sorgolactone. The Journal of Organic Chemistry 63, 1259-1267.

Surwenshi, A., Chimmad, V.P., Ravikumar, R.L., 2010. Physiological studies on hybrids and parents in relation to morpho-phenological parameters, yield and yield components in sorghum. Karnataka Journal of Agricultural Sciences 20-1.

Taylor, J. R. N. (2003). Overview: Importance of sorghum in Africa. In Afripro: Workshop on the Proteins of Sorghum and Millets: Enhancing Nutritional and Functional Properties for Africa, Pretoria (Vol. 2, No. 4).

Toh, S., Holbrook-Smith, D., Stogios, P.J., Onopriyenko, O., Lumba, S., Tsuchiya, Y., Savchenko, A., McCourt, P., 2015. Structure-function analysis identifies highly sensitive strigolactone receptors in Striga. Science 350, 203-207.

Tuinstra, M.R., Soumana, S., Al-Khatib, K., Kapran, I., Toure, A., van Ast, A., Bastiaans, L., Ochanda, N.W., Salami, I., Kayentao, M., 2009. Efficacy of herbicide seed treatments for controlling infestation of sorghum. Crop science 49, 923-929.

Van Ast, A., Bastiaans, L., Kropff, M.J., 2000. A comparative study on Striga hermonthica interaction with a sensitive and a tolerant sorghum cultivar. Weed Research 40, 479-493.

Vasudeva Rao, M.J., 1985. Techniques for screening sorghums for resistance to Striga. ICRISAT, Andhra Pradesh, pp 96-112.

Vogler, R.K., Ejeta, G., Butler, L.G., 1996. Inheritance of low production of Striga germination stimulant in sorghum. Crop science 36, 1185-1191.

Wilson Jones, K. (1953). The witchweeds of Africa. World Crops, 5(7), 263-266.

Xie, X., Yoneyama, K., Kisugi, T., Uchida, K., Ito, S., Akiyama, K., Hayashi, H., Yokota, T., Nomura, T., Yoneyama, K., 2013. Confirming stereochemical structures of strigolactones produced by rice and tobacco. Molecular plant 6, 153-163.

Xie, X., Yoneyama, K., Kusumoto, D., Yamada, Y., Takeuchi, Y., Sugimoto, Y., Yoneyama, K., 2008. Sorgomol, germination stimulant for root parasitic plants, produced by Sorghum bicolor. Tetrahedron Letters 49, 2066-2068.

Xie, X., Yoneyama, K., Yoneyama, K., 2010. The strigolactone story. Annual review of phytopathology 48, 93-117. 
Yoneyama, K., Awad, A.A., Xie, X., Yoneyama, K., Takeuchi, Y., 2010. Strigolactones as germination stimulants for root parasitic plants. Plant and Cell Physiology 51, 1095-1103.

Yoneyama, K., Xie, X., Kusumoto, D., Sekimoto, H., Sugimoto, Y., Takeuchi, Y., Yoneyama, K., 2007. Nitrogen deficiency as well as phosphorus deficiency in sorghum promotes the production and exudation of 5-deoxystrigol, the host recognition signal for arbuscular mycorrhizal fungi and root parasites. Planta 227, 125-132.

Zhang, Y., 2014. Elucidation of strigolactone biosynthesis in the host plant rice and the signal perception in the parasitic plant Striga hermonthica. PhD, Dissertation, Wageningen University, Wageningen.

Zhang, Y., van Dijk, A.D.J., Scaffidi, A., Flematti, G.R., Hofmann, M., Charnikhova, T., Verstappen, F.W.A., Hepworth, J., van der Krol, A.R., Leyser, O., 2014. Rice cytochrome P450 MAX1 homologs catalyze distinct steps in strigolactone biosynthesis. Nature Chemical Biology 10, 10281033.

Zwanenburg, B., Pospíšil, T., 2013;. Structure and activity of strigolactones: new plant hormones with a rich future. Molecular plant 6, 38-62. 


\title{
Chapter 2
}

\section{Genetic variation in Sorghum bicolor strigolactones and their role in resistance against Striga hermonthica}

Nasreldin Mohemed, Tatsiana Charnikhova, Emilie F. Fradin, Juriaan Rienstra, Abdelgabar G.T. Babiker and Harro J. Bouwmeester.

Journal of Experimental Botany, in press.

\begin{abstract}
Sorghum is an important food, feed, and industrial crop worldwide. Parasitic weeds of the genus Striga constitute a major constraint to sorghum production, particularly in the drier parts of the world. In this study we analysed the Striga germination stimulants, strigolactones, in the root exudates of 36 sorghum genotypes and assessed Striga germination and infection. Low germination-stimulating activity and low Striga infection correlated with the exudation of low amounts of 5-deoxystrigol and high amounts of orobanchol, whereas susceptibility to Striga and high germinationstimulating activity correlated with high concentrations of 5-deoxystrigol and low concentrations of orobanchol. Marker analysis suggested that similar genetics to those previously described for the resistant sorghum variety SRN39 and the susceptible variety Shanqui Red underlie these differences. This study shows that the strigolactone profile in the root exudate of sorghum has a large impact on the level of Striga infection. High concentrations of 5-deoxystrigol result in high infection, while high concentrations of orobanchol result in low infection. This knowledge should help to optimize the use of low germination stimulant-based resistance to Striga by the selection of sorghum genotypes with strigolactone profiles that favour normal growth and development, but reduce the risk of Striga infection.
\end{abstract}




\section{Introduction}

The root-parasitic weed Striga (Striga hermonthica [Del.] Benth.) is a serious threat to food security in sub-Saharan Africa. The parasite is one of the most serious pests limiting yields of the major cereal crops, maize (Zea mays L.), pearl millet [Pennisetum glaucum (L.) R. Br.], and sorghum (Sorghum bicolor [L.] Moench) (Taylor, 2003; Gressel et al., 2004; Ejeta, 2007; Scholes and Press, 2008; Badu-Apraku et al., 2013). Methods used to control Striga range from cheap traditional means such as hand pulling, crop rotation, fallow, and variety choice, employed by resource-limited farmers, to modern, more expensive interventions such as the use of herbicides and fertilizers, practised by resource-rich farmers (Oswald, 2005; Parker, 2009). For decades, the development of satisfactory, low-cost, and efficient control technologies has been a major and difficult challenge, owing to the complexity of the parasite's life cycle, its production of large amounts of tiny seeds with prolonged viability, and the serious damage inflicted on the host by the parasite while it is still hidden underground (Scholes and Press, 2008; Spallek et al., 2013). The increase in frequency and magnitude of crop yield losses and the risk of potential future spread have led to an intensification of studies aiming to improve Striga control through cultural and chemical measures and the generation of Striga-resistant crop varieties. Striga-resistant crop genotypes have been proposed to provide the simplest, cheapest, and most durable solution to the problem (Ejeta, 2005; Tesso and Ejeta, 2011).

In general, Striga resistance refers to the ability to reduce or prevent infection with and reproduction of the parasite, while tolerance refers to the ability to support equally severe levels of infection as other varieties of the same host species without the associated impairment of growth or loss in grain yield (Rodenburg et al., 2005; Rodenburg and Bastinaans, 2011). One of the most studied and best documented mechanisms of resistance against Striga is based on the fact that a host-derived signal is required for Striga seeds to germinate. Low production of the germination signal could potentially be a mechanism of resistance (Ejeta et al., 1992; Ejeta et al., 2000; Ejeta et al., 2007; Yoder and Scholes, 2010; Cardoso et al., 2011). The sorghum cultivar Framida, originating from Uganda, was reported as early as 1958 to be a low 
germination-stimulant producer (Williams, 1958). In Sudan, the sorghum cultivars Tetron and SRN39 were also reported to be low stimulant producers (Kambal and Musa, 1979; Bebawi, 1981). In addition, positive correlations between the amount of germination stimulant produced and Striga infection levels in the field were reported by several authors (Vasudeva Rao, 1984; Rich et al., 2004; Mohemed et al., 2016).

Parker et al. (1977) used the double-pot technique to screen a large collection of sorghum genotypes for low germination stimulant-based resistance to Striga asiatica. In this technique, 7-day-old sorghum seedlings were grown in sterile quartz sand in a pot with a perforated base, which was placed in another pot without perforations to collect root exudates. An aliquot of the root exudate was applied to preconditioned Striga seeds to assess its germination-inducing activity. Use of the double pot and other similar techniques resulted in the identification of several low-stimulant genotypes. Field screening for resistance to Striga in Sudan revealed that field resistance is more frequent among low stimulant producers than among high stimulant producers (Babiker, 2002). The agar gel assay was developed to screen sorghum genotypes for resistance to Striga based on the low capacity of the root exudates to stimulate the germination of Striga seeds under controlled conditions (Hess et al., 1992; Haussmann et al., 2000). The agar gel assay was also used for mapping of a low-germinationstimulant quantitative trait locus (QTL), $\lg s$, which was subsequently used as a marker to transfer the low-stimulant trait into other sorghum genotypes (Hess et al., 1992; Scholes and Press, 2008; Satish et al., 2012). In all the screening approaches described above, sorghum genotypes were identified as low or high stimulant producers on the basis of the germination-inducing activity of their root exudate rather than on the composition and nature of the signalling molecules. Identification of the sorghumderived germination stimulant has been an important research target for decades. Sorgoleone and dihydrosorgoleone were the first identified sorghum-derived Striga germination stimulants in cultivar IS 4225 (Chang et al., 1986). However, sorghum cultivars with resistance to Striga based on low stimulant production were found to produce the same amounts of these compounds as the susceptible cultivars, undermining an in planta role for sorgoleone in the induction of Striga seed germination (Siame et al., 1993). Indeed, sorghum also produces strigolactones, and these were 
found to be more active in inducing Striga germination at extremely low concentrations, and to correlate better with resistance to Striga, than dihydrosorgoleone (Bouwmeester et al., 2003; Ejeta et al., 2007; Xie et al., 2010). Strigolactones including strigol, sorgolactone, sorgomol, and 5-deoxystrigol were identified in a number of sorghum cultivars (Hauck et al., 1992; Siame et al., 1993; Awad et al., 2006; Xie et al., 2008) (Fig. 1). However, variation in the composition and quantity of strigolactones produced by different sorghum varieties have been discussed in only a few reports. For example, Awad et al. (2006) characterized 5-deoxystrigol as the major stimulant in three sorghum cultivars (M 800, Hybrid, and Swarna), sorgomol as a second major stimulant in two of them (M 800 and Hybrid), and strigol, but not sorgomol, as the second major stimulant in the root exudate of the third cultivar (Swarna). In addition, sorgolactone-which was previously isolated by Hauck et al. (1992) from the sorghum cultivar Haygrazer-was not detected in any of the cultivars investigated by Awad et al. (2006). Structural variation among the strigolactones present in root exudates of different sorghum genotypes has been suggested to influence susceptibility to Striga in the field (Yoneyama et al., 2015). For example, the Striga-susceptible sorghum cultivar Tabat exuded 5-deoxystrigol as the major stimulant (Yoneyama et al., 2010), while the Strigaresistant cultivar SRN39 exuded only small amounts of 5-deoxystrigol and had sorgomol as its major stimulant, in addition to a number of minor putative unidentified strigolactones (Yoneyama et al., 2010). The authors suggested that susceptibility to Striga of some sorghum genotypes is associated with the production and/or exudation of more stable non-hydroxy strigolactones, that is, 5-deoxystrigol and sorgolactone, rather than the more unstable strigolactone alcohols such as sorgomol (Yoneyama et al., 2010). Although strigolactones have been analysed in sorghum root exudates and genotypic variation for strigolactone exudation in sorghum has been demonstrated, more studies on the direct association between host genotype, strigolactone exudation, and Striga germination, host specificity, and parasitism are still needed.

The present investigation, which included 36 sorghum genotypes comprising landraces and improved varieties collected from Sudan, was carried out to study the influence of genotypic variation in the quality and quantity of strigolactones in the sorghum root exudates and their role in Striga infection. 


\section{Materials and methods}

Seeds of 36 sorghum genotypes were obtained from the gene bank of the Agricultural Research Co-operation (ARC) Wad Medani, Sudan (see Supplementary Table S1). Data on field performance, agronomic traits, and susceptibility to Striga were obtained from the sorghum breeding program of the ARC. Seeds of a sorghum ecotype of Striga hermonthica (Del.) Benth. were collected in 2009/2010 in a sorghum field at the Abu Naama Research Station, Sudan, and were supplied by Dr A. Hamid, Sinnar University, Sudan.

\section{Analysis of root exudates}

For the collection of root exudates, germinated sorghum seeds of the 36 genotypes were planted in 3 litre plastic pots filled with 1.5 litres of sand. One week after planting, the seedlings were thinned to five plants per pot. Half-strength modified Hoagland's nutrient solution was applied to each pot (500 $\mathrm{ml}$ at $48 \mathrm{~h}$ intervals). The plants were allowed to grow under controlled conditions in a climate room with artificial light at $450 \mu \mathrm{mol} \mathrm{m}{ }^{-2} \mathrm{~s}^{-1}$ and at a temperature of $28^{\circ} \mathrm{C}$ (day; $10 \mathrm{~h}$ ) $/ 25^{\circ} \mathrm{C}$ (night; $14 \mathrm{~h}$ ) and $70 \%$ relative humidity for 4 weeks. In the fifth week, phosphorus (P) deficiency was created in each pot to increase strigolactone production (López-Ráez et al., 2008). To achieve this, 3 litres of P-deficient nutrient solution (half-strength modified Hoagland's nutrient solution minus P) were added to each pot and allowed to drain freely from the pot. The plants were kept under P deficiency for 1 week prior to flushing each pot with 3 litres of P-deficient nutrient solution to remove accumulated strigolactones. The plants were then allowed to grow for an additional $48 \mathrm{~h}$, after which root exudates were collected in a 1 litre plastic bottle by passing 3 litres of nutrient solution without $P$ through each pot. The collected exudate was passed over a solid phase extraction (SPE) C18 column (500 $\mathrm{mg} 3 \mathrm{ml}^{-1}$ ) and strigolactones were eluted with $6 \mathrm{ml}$ acetone. For further purification, the acetone was evaporated under a vacuum at $25{ }^{\circ} \mathrm{C}$ using a rotary evaporator. The residue was dissolved in $4 \mathrm{ml}$ hexane and loaded on to a pre-equilibrated Silica gel Grace Pure SPE (200 mg $3 \mathrm{ml}^{-1}$ ) column; strigolactones were eluted with $2 \mathrm{ml}$ hexane:ethyl acetate (1:9). The solvent was evaporated and the residue dissolved in 200 $\mu 1$ of $25 \%$ aqueous acetonitrile. The sample was filtered through a Minisart SRP4 0.45 $\mu \mathrm{m}$ filter (Sartorius, Germany) prior to LC-MS/MS analysis. 


\section{Strigolactone analysis}

The strigolactones 5-deoxystrigol, sorgomol, and orobanchol were identified and quantified using ultra-performance liquid chromatography-tandem mass spectrometry (UPLC-MS/MS) as previously described by López-Ráez et al. (2008). The samples were analysed by a Waters Xevo triple quadrupole tandem mass spectrometer (Waters, Milford, MA, USA) equipped with electrospray ionization source and coupled to an Acquity UPLC system (Waters). Multiple reactions monitoring (MRM) was used for quantification of strigolactones in sorghum root exudates. 5-Deoxystrigol was detected at a retention time of $7.8 \mathrm{~min}$ in MRM channels $m / z$ 331>97, 331>216, and 331>234; sorgomol at $4.8 \mathrm{~min}$ in MRM channels $m / z$ 347>317, 317>97, and 317>133; orobanchol at $4.5 \mathrm{~min}$ in MRM channels $m / z$ 347>97, 347>205, and 347>233; sorgolactone at 4.7$4.8 \mathrm{~min}$ in MRM channels $\mathrm{m} / \mathrm{z} 317>97$ and $317>133$; strigol at $4.50 \mathrm{~min}$ in MRM channels $m / z$ 347>97, 329>97, and 329>215; ent-2'-epi-orobanchol at $4.5 \mathrm{~min}$ in MRM channels $m / z \quad 347>97,347>205$, and 347>233; and ent-2'-epi-5-deoxystrigol at $8.0 \mathrm{~min}$ in MRM channels $m / z$ 331>97, 331>216, and 331>234. Data acquisition and analysis were performed using Mass Lynx 4.1 (TargetLynx) software (Waters).

\section{Assessment of germination stimulant activity}

Striga seeds placed on $8 \mathrm{~mm}$ glass fibre filter paper discs ( $\sim 50$ seeds each) were surface sterilized and preconditioned as previously described by Matusova et al. (2004). The discs containing preconditioned seeds were treated with aliquots $(50 \mu 1)$ of authentic strigolactones (5-deoxystrigol, sorgomol, orobanchol, strigol, sorgolactone, ent-2'-epi5-deoxystrigol, and ent-2'-epi-orobanchol) $(0.02 \mu \mathrm{M}$ in water) or a 100-fold diluted, C18-purified root exudate, and then incubated at $30{ }^{\circ} \mathrm{C}$ for $48 \mathrm{~h}$ and subsequently examined for germination (radicle protrusion). Distilled water and the synthetic strigolactone GR24 $(0.02 \mu \mathrm{M})$ were included as negative and positive controls, respectively. All treatments were replicated six times.

\section{Assessment of Striga infection}

Based on differences in strigolactone production and profile, 22 sorghum genotypes were selected for a pot experiment using soil infested with Striga seeds to examine whether differences in strigolactone content in the exudate resulted in differences in Striga infection. Plants were grown in 18 litre pots $(0.25 \mathrm{~m}$ length $\times 0.25 \mathrm{~m}$ width $\times$ 
$0.30 \mathrm{~m}$ height) containing a mixture of sand and soil, collected from the top layer ( 0 $0.25 \mathrm{~m}$ ) of an arable field near Wageningen. Striga seeds $(8 \mathrm{mg})$ were added and mixed thoroughly with the soil in each pot. The pots were watered and kept for 10 days in the greenhouse to allow for conditioning of Striga seeds. Subsequently, five seeds of each of the 22 sorghum genotypes were sown in the middle of the pot. Seedlings were thinned to one plant per pot 4 days after emergence. Plants were grown in a temperaturecontrolled greenhouse at a temperature of $28{ }^{\circ} \mathrm{C}$ (day; $\left.10 \mathrm{~h}\right) / 25^{\circ} \mathrm{C}$ (night; $14 \mathrm{~h}$ ), with natural sunlight supplemented with artificial light and at $70 \%$ relative humidity. Halfstrength modified Hoagland's nutrient solution was applied in the first week ( $250 \mathrm{ml}$ at $48 \mathrm{~h}$ intervals). For the remainder of the experimental period, a nutrient solution with $20 \% \mathrm{P}(250 \mathrm{ml}$ per pot at $48 \mathrm{~h}$ intervals $)$ was applied to stimulate strigolactone exudation.

Counting of Striga seedlings started when Striga emergence was first observed on the susceptible genotype Tabat, $\sim 2$ weeks after sowing. Subsequently, the number of emerged Striga seedlings per pot per genotype was assessed every 3 days until Striga had emerged on all of the 22 genotypes. Further Striga counts were made at weekly intervals for 10 weeks. The total number of Striga plants per host plant was determined after harvesting each genotype at maturity by root washing and counting the attached Striga tubercles and plants.

\section{Gene expression analysis}

To investigate the role of strigolactone biosynthetic genes as a possible explanation for genotypic differences in strigolactone production among sorghum genotypes, we selected three resistant genotypes, SRN39, IS9830, and Tetron, which produce low amounts of 5-deoxystrigol and sorgomol but higher amounts of orobanchol, and which induce low Striga germination and exhibit low levels of Striga infection; and one susceptible genotype, Fakimustahi, which produces high amounts of 5-deoxystrigol and sorgomol but low amounts of orobanchol, and which elicits high Striga seed germination and displays high levels of Striga infection. Differences in the expression of the sorghum orthologs of the rice strigolactone biosynthetic genes DWARF27 (Sb05g022855.1), DWARF17 (CAROTENOID CLEAVAGE DIOXYGENASE7; CCD7) (Sb06g024560), DWARF10 (CAROTENOID CLEAVAGE DIOXYGENASE 8; CCD8) 
(Sb03g034400.1), and MORE AXILLARY BRANCHING 1 (MAX1) (Sb03g032220) were analysed for these genotypes (Supplementary Tables S2-S5).

Primers were designed on the basis of the predicted mRNA sequences (Supplementary Table S6) in the National Center for Biotechnology Information (NCBI) database. The D27 (DWARF27) gene sequence was obtained from the published sequence in the NCBI database (http://www.ncbi.nlm.nih.gov; clone ID-FJ641055) and the corresponding sorghum orthologous gene was identified by BLASTn analysis (Supplementary Table S2). For SbCCD 8 and SbCCD7, the corresponding rice gene sequence was used to identify the orthologs in sorghum (http://www.gramene.org) as described previously (Vallabhaneni et al., 2010; Guan et al., 2012; Priya and Siva, 2014) (Supplementary Tables S3 and S4). For SbMAX1, Arabidopsis thaliana MAX1 (AT2G26170) was obtained from the published sequence (http://www.arabidopsis.org) and the corresponding sorghum orthologous gene was identified by BLASTn analysis in PlantGDB (http://www.plantgdb.org/) (Supplementary Table S5). Primer pairs for individual genes were designed with Primer Express 2.0 software (Applied Biosystems, Foster City, CA, USA). Primer sequences were confirmed using the BLASTn program to ensure amplification of unique and appropriate cDNA fragments (Supplementary Table S6).

Total RNA was extracted from roots of the genotypes that were grown under conditions of P deprivation for 2 weeks. The RNA was extracted from $150 \mathrm{mg}$ of homogenized ground roots using $500 \mathrm{ml}$ Trizol (Invitrogen) and subsequently purified with chloroform and precipitated with isopropanol. Pellets were washed with ethanol $(70 \%$ $\mathrm{v} / \mathrm{v}$ ) and then resuspended in $20 \mu \mathrm{l}$ Milli-Q water, and DNA was removed using a DNAase I Kit (Qiagen) according to the manufacturer's instructions. The RNA was cleaned by using a DNase treatment prior to quantitative (q) PCR (Promega).

Gene expression was assessed by using cDNA from each sorghum genotype. cDNA was synthesized by using the iScript cDNA Synthesis Kit (BioRad) using $1 \mu \mathrm{g}$ of total RNA per sample, following the manufacturer's instructions. The qPCR reactions were performed using iQ SYBR Green Supermix (BioRad). The qPCR reaction consisted of $5 \mu \mathrm{l}$ of SYBR Green Supermix, $0.5 \mu 1$ of forward primer, $0.5 \mu 1$ of reverse primer (with each primer at a concentration of $0.1 \mu \mathrm{M}), 1 \mu \mathrm{l}$ of 10 -fold diluted template cDNA, and 
$2 \mu \mathrm{l}$ double-deionized water. The qRT-PCR program was one cycle of $95^{\circ} \mathrm{C}$ for $10 \mathrm{~s}$, followed by 45 cycles of $94{ }^{\circ} \mathrm{C}$ for $10 \mathrm{~s}, 60{ }^{\circ} \mathrm{C}$ for $10 \mathrm{~s}$, and $72{ }^{\circ} \mathrm{C}$ for $15 \mathrm{~s}$. The amplifications were detected by using a BioRad CFX Connect. The relative levels of RNA for each gene were calculated from cycle threshold values according to the $\Delta \Delta \mathrm{Ct}$ method (Schmittgen and Livak, 2008). The specificity of the reactions was verified by melting curve analysis. The expression data presented are the average of three control and three biological replicates. The sorghum actin gene SbACTIN (Sb01g010030.1) (Supplementary Table S6) was used as a reference.

\section{Marker analysis}

PCR was performed in a total reaction volume of $25 \mu 1$. The reaction was set up as follows: sterile distilled water $14.76 \mu$, buffer $(5 \times) 5 \mu$, forward primer $(10 \mathrm{mM}) 0.5$ $\mu 1$, reverse primer $(10 \mathrm{mM}) 0.5 \mu 1$, dNTPs $(10 \mathrm{mM}) 0.5 \mu 1, \mathrm{MgCl}_{2}(25 \mathrm{mM}) 2.5 \mu 1$, Taq DNA polymerase (5U) $0.24 \mu \mathrm{l}$, and template DNA $\left(50 \mathrm{ng} \mu \mathrm{l}^{-1}\right) 1 \mu \mathrm{l}$. PCR amplification conditions were as follows: initial denaturation at $94{ }^{\circ} \mathrm{C}$ for $5 \mathrm{~min}$, followed by 40 cycles of denaturation at $94^{\circ} \mathrm{C}$ for $1 \mathrm{~min}$, annealing at $50{ }^{\circ} \mathrm{C}$ for $1 \mathrm{~min}$, and elongation at 72 ${ }^{\circ} \mathrm{C}$ for 2 min., followed by a final elongation step at $72{ }^{\circ} \mathrm{C}$ for $5 \mathrm{~min}$. The reaction

products were loaded on to $3 \%$ agarose gels, stained with ethidium bromide, and run in $1 \times$ Tris-acetate-EDTA buffer at $100 \mathrm{~V}$ for $1 \mathrm{~h}$, with a $50 \mathrm{bp}$ DNA (0.5 $\mu \mathrm{g}$ per lane) ladder (Thermo Scientific). Gels were visualized using an EpiChemi II Darkroom (UVP Ltd, Cambridge, UK) gel documentation system. The sequences of the forward and reverse primers for the SB3344 markers are provided in Supplementary Table S7.

\section{Statistical analysis}

The statistical package SAS (version 15) was used for analysis of variance (ANOVA) and Pearson's correlation analyses. Duncan's honest significant difference test was subsequently performed to establish the significance of differences. The relationship between various strigolactones and the number of emerged Striga seedlings, Striga biomass, and in vitro Striga germination was analysed by correlation analysis and stepwise regressions using the IBM SPSS Statistics 20 package. The Origin Pro 9 (64bit) statistical software package was used for principal component analysis (PCA). To 
meet the assumption of normality, the strigolactone peak area and number of emerged Striga seedlings were subjected to logarithmic transformation prior to ANOVA.

\section{Results}

The major strigolactones that were detected in the root exudates of all sorghum genotypes under investigation were 5-deoxystrigol, sorgomol, and orobanchol (Fig. 1; Supplementary Table S8). Other strigolactones, including sorgolactone, strigol, ent-2'epi-orobanchol and ent-2'-epi-5-deoxystrigol, were detected in low concentrations in the root exudates of some genotypes (data not shown). The amount of strigolactones secreted and the ratio between the individual strigolactones differed considerably among sorghum genotypes (Supplementary Table S8; Fig. 2). Among all the genotypes studied, Debeikri, Feterita Geshaish, Hemisi, Kolom, Najad, Tabat, and N13 were the highest 5-deoxystrigol producers (Supplementary Table S8). Bari, Dari, Fakimustahi, Wadfahel, and Wad Elmardi were the highest sorgomol producers, and SRN39, Tetron, IS9830, Framida, and Hakika were the highest orobanchol producers (Supplementary Table S8). The latter genotypes were also among the lowest 5-deoxystrigol producers. Arfa Gadamak, Korokollow, Mogud, Tafsagabeid, Tokarawe, Wad Ahmed, and Wadbaco secreted intermediate amounts of 5-deoxystrigol and low amounts of sorgomol and orobanchol (Supplementary Table S8). 


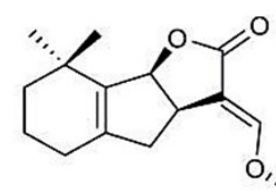

5-deoxystrigol<smiles>CC1(C)CCC(O)C2=C1C1CC3/C(=C\O)C(=O)OC3C21</smiles>

strigol

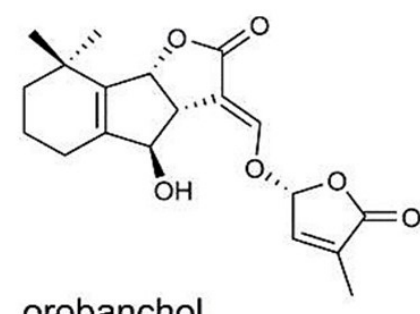

orobanchol<smiles>C[C@H]1CCCC2=C1C1OC(=O)/C(=C/O)C1C2</smiles>

sorgolactone

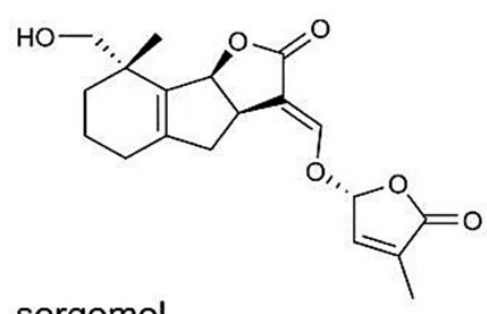

sorgomol

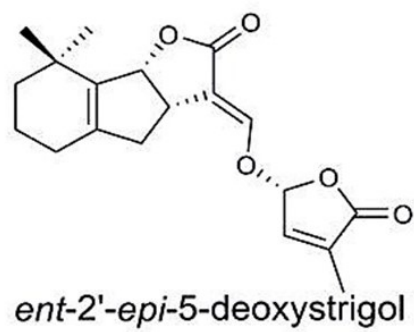<smiles>CC1(C)CCCC2=C1[C@H]1OC(=O)/C(=C/O)[C@H]1[C@H]2O</smiles>

ent-2'-epi-orobanchol<smiles>CC1=CCOC1=O</smiles><smiles>CCO[C@H]1C=C(C)C(=O)O1</smiles> 
and Debeikri; low (20-24\%) in Gadam Elhamam, Tabat, N13, Naten, Zahrat Elgadambalia, Aklamoi, Bari, Dari, Tokarawe, Wad Elmardi, Kolom, Markoob, Feterita, Hazaztokarwe, and Hariri; and very low (2-9\%) in Wadfahel, Baham, Tafsagabeid, and Fakimustahi (Fig. 2). The relative proportion of sorgomol in the strigolactone blend was high (40-44\%) in Hariri, Bari, Fakimustahi, Dari, and Naten; moderate (29-38\%) in Wadfahel, Hakika, Wad Elmardi, IS15401, Wadbaco, Zahrat Elgadambalia, Aklamoi, Hemisi, Tokarawe, Najad, SRN39, Tabat, and N13; moderately low (24-28\%) in Markoob, Gadam Elhamam, Wad Ahmed, Feterita Geshaish, and Framida; low (16-23\%) in Dabar, Hazaztokarwe, Tafsagabeid, Debeikri, Kolom, Botana, Feterita, Baham, and Mogud; and very low (2-9\%) in Korokollow, IS9830, Arfa Gadamak, and Tetron (Fig. 2).

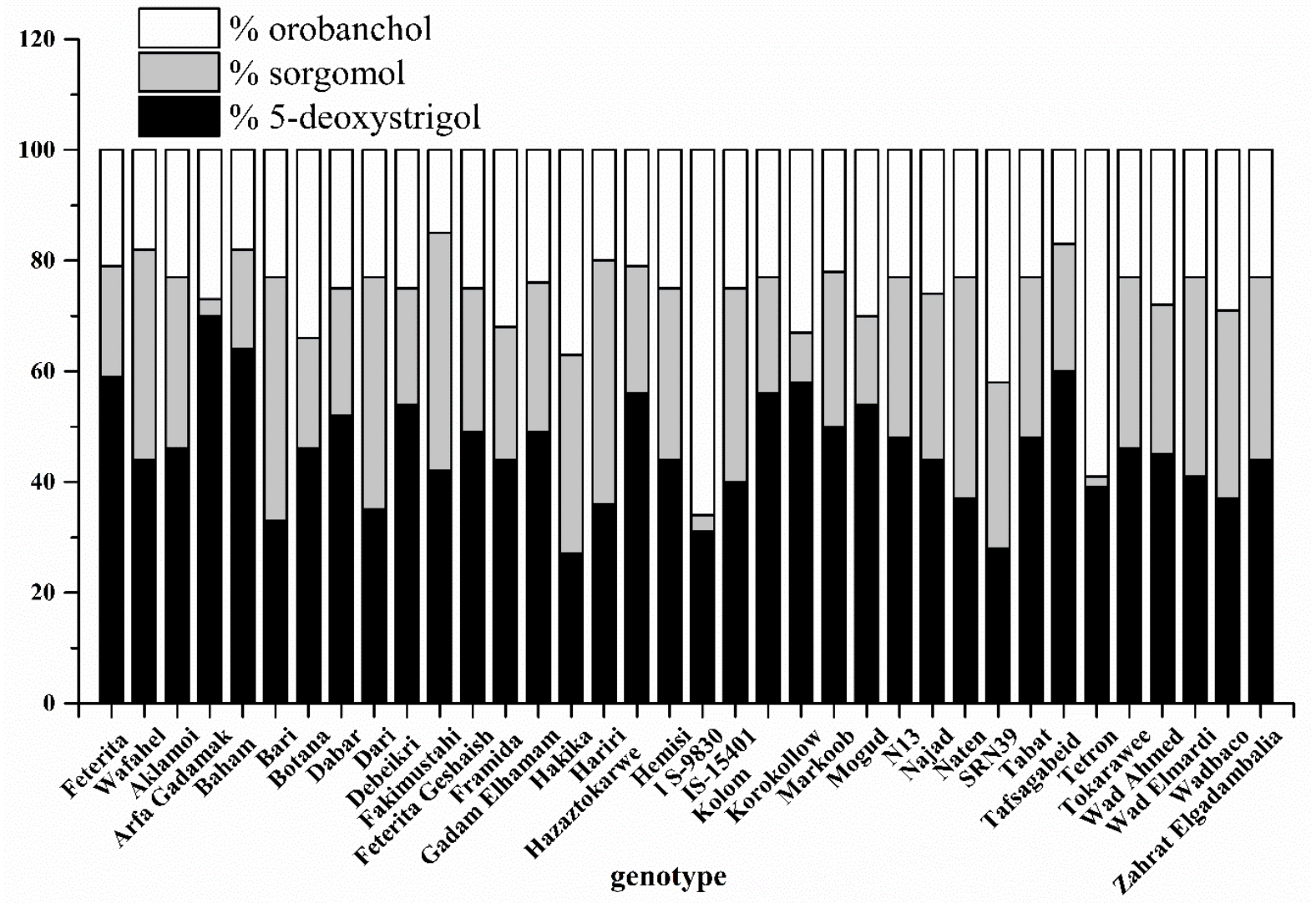

Fig. 2. Genotypic variation in the proportions of the strigolactones 5-deoxystrigol, sorgomol, and orobanchol in root exudates of sorghum.

\section{Germination-inducing activity of sorghum root exudates}

The C18-purified, 100-fold-diluted root exudates displayed significant variation in germination-inducing activity $(P<0.01)$ (Fig. 3). The germination-inducing activity of root exudates was very high (60-67\%) for Bari, Dabar, Dari, Fakimustahi, Feterita 
Geshaish, Kolom, Markoob, N13, Tabat, and Wad Elmardi; high (50-59\%) for Debeikri, Feterita, Hemisi, Gadam Elhamam and Hazaztokarwe; moderate (30-49\%) for Baham, Tafsagabeid, Zahrat Elgadambalia, Wad Ahmed, Naten, Arfa Gadamak, Najad, IS15104, IS9830, Tetron, Hariri, Mogud, and Korokollow; and low (0-29\%) for Aklamoi, Botana, Framida, Hakika, SRN39, Wadbaco, Wadfahel and Tokarawe. Among the genotypes, the germination-inducing activity of the root exudates was highest for Wad Elmardi, Fakimustahi, and Tabat, and lowest for SRN39, Wadbaco, and Framida (Fig. 3). Distilled water and the synthetic strigolactone GR24 (0.02 $\mu \mathrm{M})$ induced negligible (0\%) and 50\% germination, respectively.

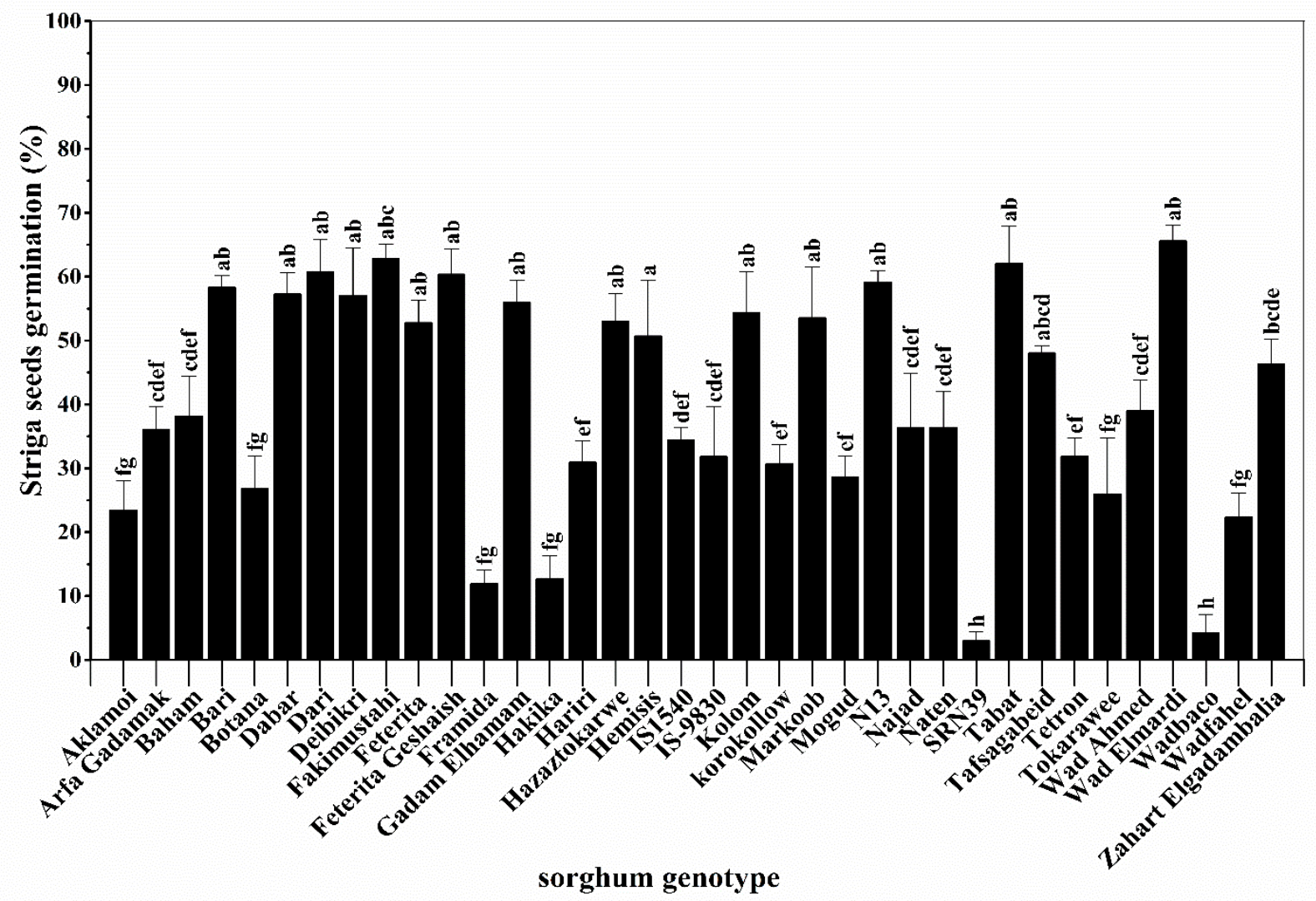

Fig. 3. Genotypic variation in the germination-inducing activity of sorghum root exudates. Bars represent means \pm SE $(n=5)$. Least significant differences of means at $P=0.05$ by ANOVA. Letters above the bars indicate different significance groups after Duncan's pairwise comparisons $(P<0.05)$. 


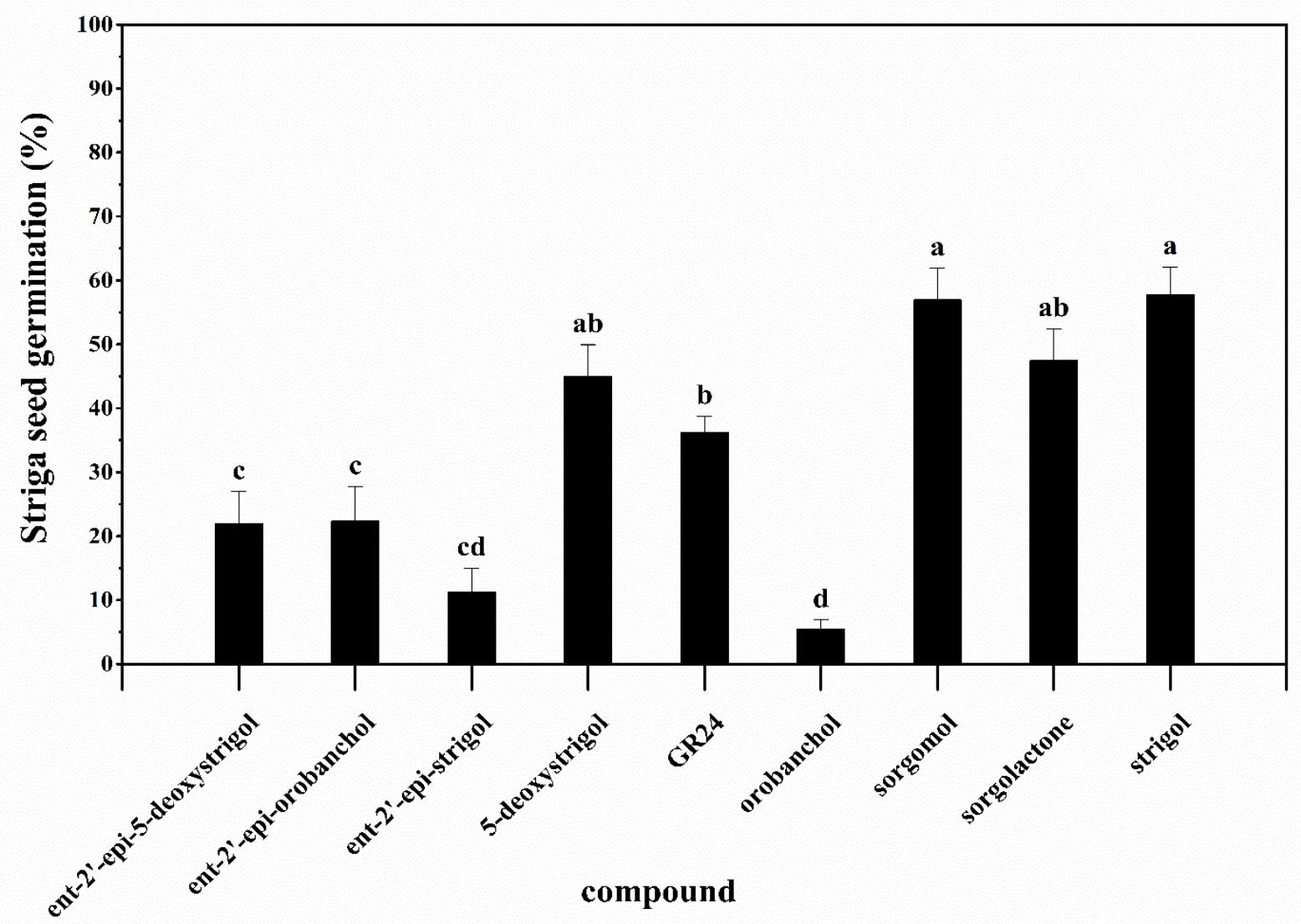

Fig. 4. Germination response of Striga seeds to natural and synthetic strigolactones at $0.02 \mu \mathrm{M}$. Results represent the mean of five replicates. Error bars indicate SE. Different letters above the bars indicate significant differences between mean values $(P<0.05$; ANOVA $)$.

\section{Germination-stimulating activity of standard strigolactones}

The natural strigolactones 5-deoxystrigol, sorgomol, orobanchol, strigol, sorgolactone, ent-2'-epi-5-deoxystrigol, and ent-2'-epi-orobanchol, together with the synthetic strigolactone GR24, each at $0.02 \mu \mathrm{M}$, displayed significant differences in germinationinducing activity (Fig. 4). GR24 induced 36\% germination. Sorgomol, strigol, sorgolactone and 5-deoxystrigol induced 57\%, 58\%, 47\%, and 45\% germination, respectively. Ent-2'-epi-orobanchol, ent-2'-epi-5-deoxystrigol, and ent-2'-epi-strigol induced $22 \%, 22 \%$, and $11 \%$ germination, respectively. Orobanchol, by contrast, induced only $5 \%$ germination. 

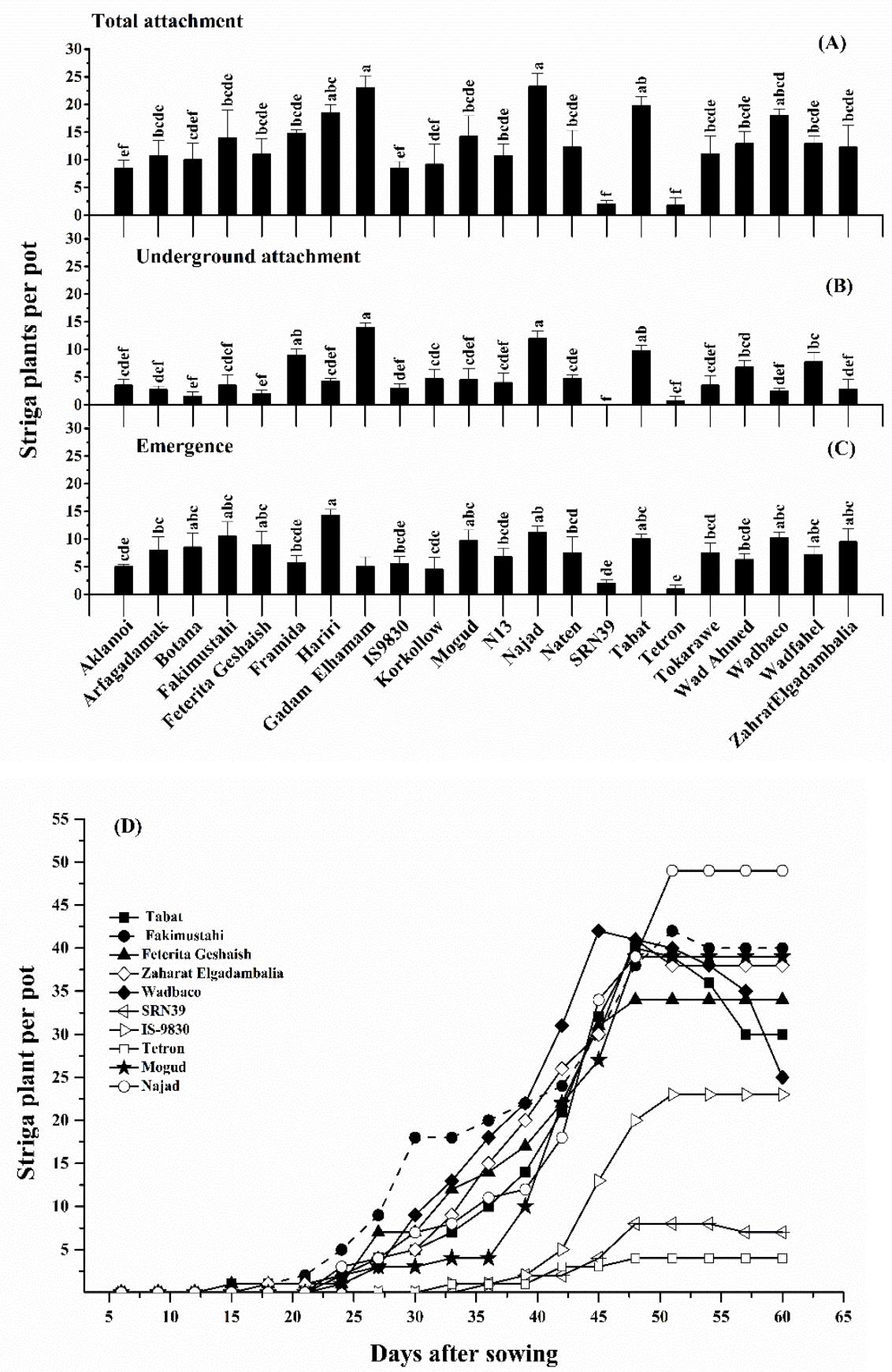

Fig. 5. Emergence of Striga plants in a greenhouse pot experiment. (A-C) Mean \pm SE total attached (A), below-ground attached, (B) and emerged (C) Striga plants per pot at harvest ( $n=4)$. The significance of a treatment effect was determined by one-way ANOVA for all genotypes; different letters above the bars indicate significant differences after Duncan's pairwise comparison $(P<0.05)$. (D) Time course of Striga emergence in the greenhouse pot experiment on 10 selected sorghum genotypes. Data are the mean total emergence of Striga plants per pot during a period of 76 days ( $n=4$ replicates). 


\section{Striga infection}

To investigate the effect of the observed differences in germination-stimulating activity on Striga infection, a selection of 22 sorghum genotypes was grown in pots with soil infected with Striga seeds. The mean total number of attached Striga plants per pot was highest (18-24) on Najad, Gadam Elhamam, Tabat, Hariri, and Wad baco; moderate (11-15) on Framida, Mogud, Fakimustahi, Wad Ahmed, Wadfahel, Naten, Zahrat Elgadambalia, Feterita Geshaish, Tokarawe, Arfa Gadamak, and N13; low (9-10) on Botana, Korokollow, and Aklamoi; and IS9830; and negligible (2) on SRN39 and Tetron (Fig. 5A).

The number of non-emerged attached Striga plants per pot was highest (9-15) on Gadam Elhamam, Najad, Tabat, and Framida; moderate (7-8) on Wad Ahmed and Wadfahel; low (3-5) on Naten, Korokollow, Mogud, Hariri, N13, Aklamoi, Fakimustahi, Tokarawe, IS9830, Arfa Gadamak, Zahrat Elgadambalia, Wadbaco, and Feterita Geshaish; and negligible (0-2) on Botana, Tetron, and SRN39 (Fig. 5B).

The number of emerged Striga plants per pot was highest (9-15) on Hariri, Najad, Fakimustahi, Wadbaco, Tabat, Mogud, Zahrat Elgadambalia, Feterita Geshaish, Botana, and Arfa Gadamak; moderate (6-8) on Naten, Tokarawe, Wadfahel, N13, Wad Ahmed, Framida, and IS9830; low (5) on Aklamoi, Korokollow, and Gadam Elhamam; and negligible (1-2) on SRN39 and Tetron (Fig. 5C).

\section{Emergence time}

The number of days to first emergence of Striga showed significant $(P<0.01)$ dependence on the sorghum genotype (Supplementary Table S9). Emergence was very early [15-18 days after sowing (DAS)] on Aklamoi, Fakimustahi, Feterita Geshaish, Tabat, Gadam Elhamam, Wadfahel, and Zahrat Elgadambalia; moderately early (23-27 DAS) on Arfa Gadamak, Botana, Framida, Korokollow, Mogud, Naten, N13, Najad, Tokarawe, and Wadbaco; and late (31-36 DAS) on Hariri, IS9830, SRN39, Tetron, and Wad Ahmed. Fig. 5D shows the emergence of Striga for a selection of 10 genotypes. 


\section{Principal component analysis}

The peak areas of 5-deoxystrigol, sorgomol, and orobanchol were used in PCA to visualize the relationship between the different sorghum genotypes, their strigolactone profile, and the germination-inducing activity of their root exudates (Fig. 6A) and, for a subset of the genotypes, the number of Striga per pot (Fig. 6B). The first two principal components in Fig. 6A had an eigenvalue higher than 1, and explained $74 \%$ of the variation in strigolactone content (Supplementary Table S10). The first principal component (PC1) explained 47\% of the variation, with positive loadings for both 5deoxystrigol and germination and a negative loading for orobanchol (Supplementary Table S10). The second principal component (PC2) explained $27 \%$ of the total variation, with a high positive loading for sorgomol (Supplementary Table S10). Along PC1, genotypes are separated based on 5-deoxystrigol and orobanchol concentration, with the highest 5-deoxystrigol-producing genotypes, such as Debeikri, Fakimustahi, Hazaztokarwe, N13, Tabat, and Wadfahel, clustering on the right side of the plot, and the highest orobanchol-producing genotypes, such as Hakika, Framida, SRN39, and Wadbaco, clustering in the upper left quadrant (Fig. 6A). The PC2 differentiated genotypes with high and low concentrations of sorgomol. The high sorgomol producers (Bari, Dari, and Wad Elmardi) clustered in the upper part of the PCA, while lowsorgomol-producing genotypes, for example, Arfa Gadamak, Korokollow, Mogud, Baham, and Dabar, clustered in the lower part.

A PCA plot of the selection of genotypes that was assessed in a pot experiment for Striga infection showed a similar clustering of genotypes, as it was based on the same strigolactone data (Fig. 6B). There was a striking strong correlation between the vectors for germination (in root exudate) and number of Striga plants per pot. According to the directions and angles of the vectors, 5-deoxystrigol exhibited a positive correlation with the germination-stimulating activity of the root exudates, while orobanchol exhibited a negative correlation with the germination-stimulating activity (Fig. 6A, B). The perpendicular angle between sorgomol and germination-stimulating activity suggests no correlation (Fig. 6A, B). The angle between 5-deoxystrigol and Striga plants per pot suggests a positive correlation, while that between orobanchol and Striga plants per pot suggests a negative correlation (Fig. 6B). 


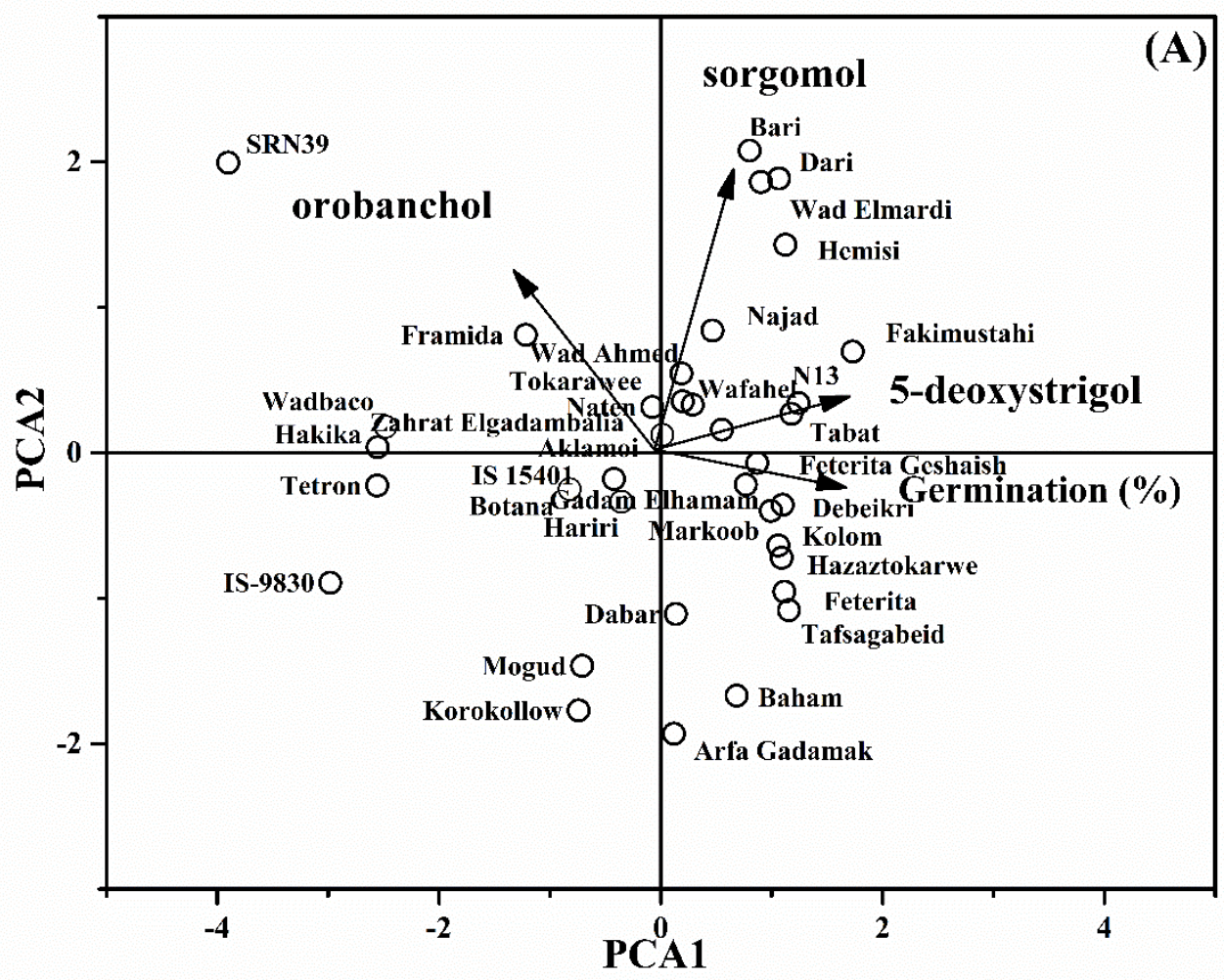




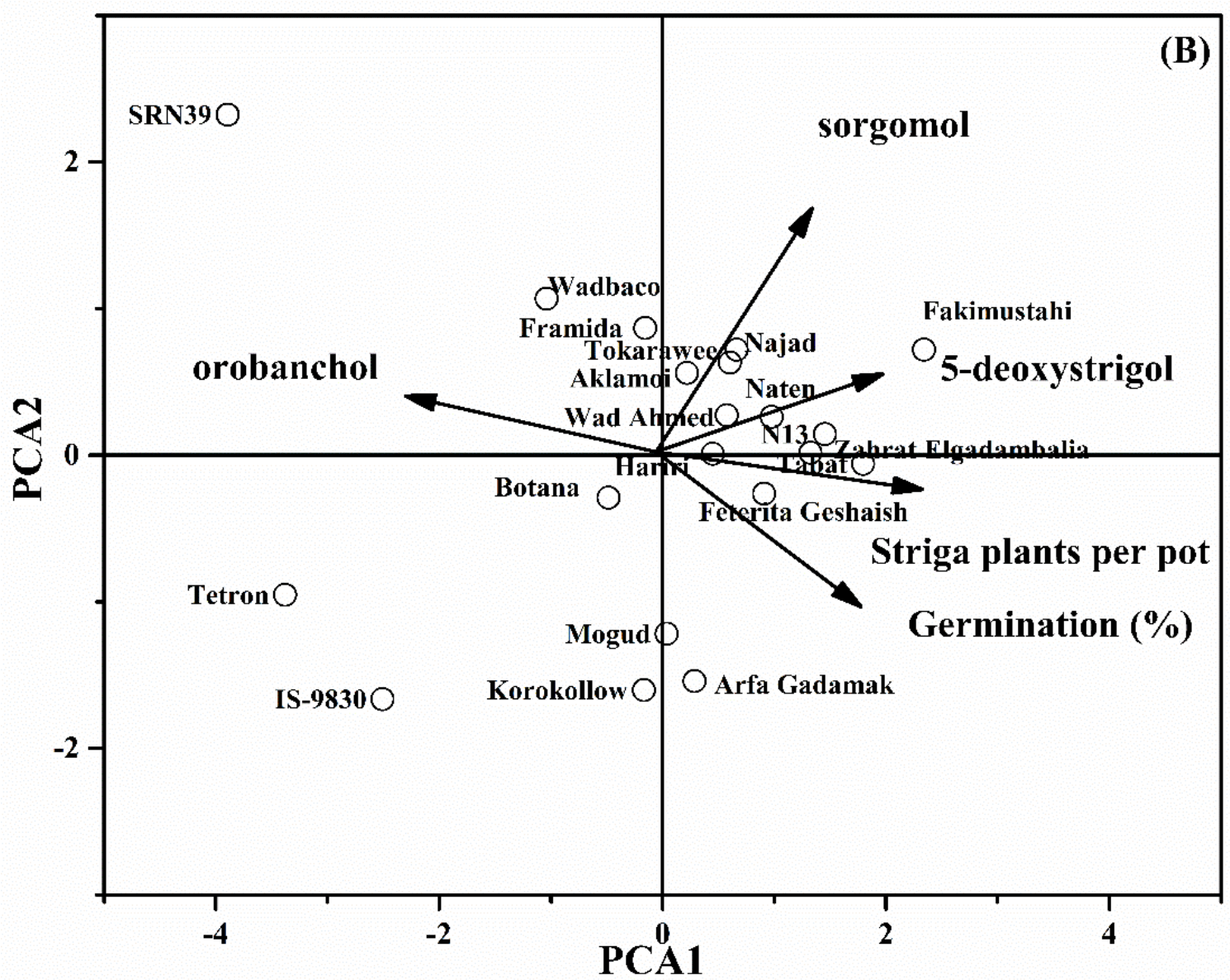

Fig. 6. (A) Bi-plot of the first two components of a PCA based on strigolactone peak area, displaying the genotypic differences in strigolactone production and composition and the correlation between the different strigolactones and germination-stimulating activity. (B) PCA showing the relationship between strigolactone production and composition in selected sorghum genotypes grown in pots, germination of Striga seeds as induced by root exudates, and the number of emergent Striga plants per pot on these genotypes.

\section{Correlation analysis}

In addition to multivariate PCA, correlation analysis was used to investigate the relationship between the concentrations of 5-deoxystrigol, sorgomol, and orobanchol in root exudates, and Striga germination and infection. The amount of 5-deoxystrigol in the root exudates displayed a significant positive correlation with Striga seed germination $(r=0.38 ; P<0.01)$, while orobanchol showed a significant negative correlation $(r=-0.25 ; \quad P<0.01)$ and sorgomol showed a non-significant negative correlation (Table 1). 
Correlation analysis across all 22 genotypes between strigolactone peak areas in the root exudate and number of emerged Striga plants in the pot experiment showed that 5deoxystrigol significantly and positively correlated with the number of emerged Striga $(r=0.36 ; P<0.01)$, while orobanchol displayed a significant negative correlation ( $r=$ $0.41 ; P \leq 0.05)$ and sorgomol a significant positive correlation $(r=0.23 ; P<0.05)$ (Table $1)$.

Table 1. Correlation coefficients between strigolactone levels in the root exudates, in vitro Striga germination, and Striga emergence in a pot experiment using 22 sorghum genotypes.

\begin{tabular}{llll}
\hline & 5-deoxystrigol & sorgomol & orobanchol \\
\hline Germination & $0.38^{* *}$ & $-0.17 \mathrm{NS}$ & $-0.25^{* *}$ \\
Striga plants per pot & $0.36^{* *}$ & $0.23^{*}$ & $-0.41^{* *}$ \\
5-deoxystrigol & 1 & $0.19^{*}$ & $-0.32^{* *}$ \\
sorgomol & $0.19^{*}$ & 1 & $-0.12 \mathrm{NS}$ \\
orobanchol & $-0.32^{* *}$ & $-0.12 \mathrm{NS}$ & 1 \\
\hline
\end{tabular}

${ }^{* *}$ Correlation is significant at the $P<0.01$ level (one-tailed). NS, not significant.

Stepwise regression with the peak areas of the strigolactones as predictors for Striga emergence resulted in a model $\left(R^{2}=0.27 ; P \leq 0.001\right)$ (Table 2$)$ containing 5-deoxystrigol, sorgomol, and orobanchol as significant predictors for Striga emergence. Of these, 5deoxystrigol showed the highest positive contribution, with a regression coefficient of $0.29(P \leq 0.006)$ (Table 2). The regression coefficients for sorgomol and orobanchol were $0.25(P \leq 0.01)$ and $-0.26(P \leq 0.01)$, respectively (Table 2$)$. 
Table 2. Summary for the stepwise best regression model $\left(R^{2}=0.27\right)$ predicting the contribution of the strigolactone level in the root exudates of sorghum genotypes to Striga emergence.

\begin{tabular}{lcc}
\hline Variables & Coefficients of regression & $\boldsymbol{P}$ \\
\hline 5-deoxystrigol & 0.29 & 0.006 \\
sorgomol & 0.25 & 0.01 \\
orobanchol & -0.26 & 0.01 \\
\hline
\end{tabular}

\section{Variation in strigolactone biosynthetic genes}

To assess the relationship between expression of strigolactone biosynthetic genes and differences in the strigolactone profile, induction of Striga seed germination, and Striga infection, we analysed four sorghum genotypes differing in Striga resistance (Fig. 7A). RT-qPCR showed that the expression of $D 27, C C D 7$, and $C C D 8$ differed significantly $(P<0.025)$ among the genotypes (Fig. 7B). The expression of both D27 and CCD 8 was higher in the Striga-susceptible genotype Fakimustahi than in the Striga-resistant genotypes IS9830, Tetron, and SRN39 (Fig. 7B). In contrast, the expression of CCD7 was higher in genotypes IS9830 and Tetron than in Fakimustahi (Fig. 7B). Although there was no significant difference in the expression of $M A X 1$ among the genotypes, it showed a tendency towards the same pattern of expression as $C C D 7$, that is, higher in the more resistant genotypes. The amount of 5-deoxystrigol and sorgomol in the exudate correlated positively with the expression of $D 27(r=0.70 ; P<0.05$, and $\mathrm{r}=0.73 ; P<0.05)$ and negatively with that of $C C D 8$ (significant only for 5-deoxystrigol: $r=-0.80 ; P<0.01$ ) (Supplementary Table S11, Fig. 7B). 

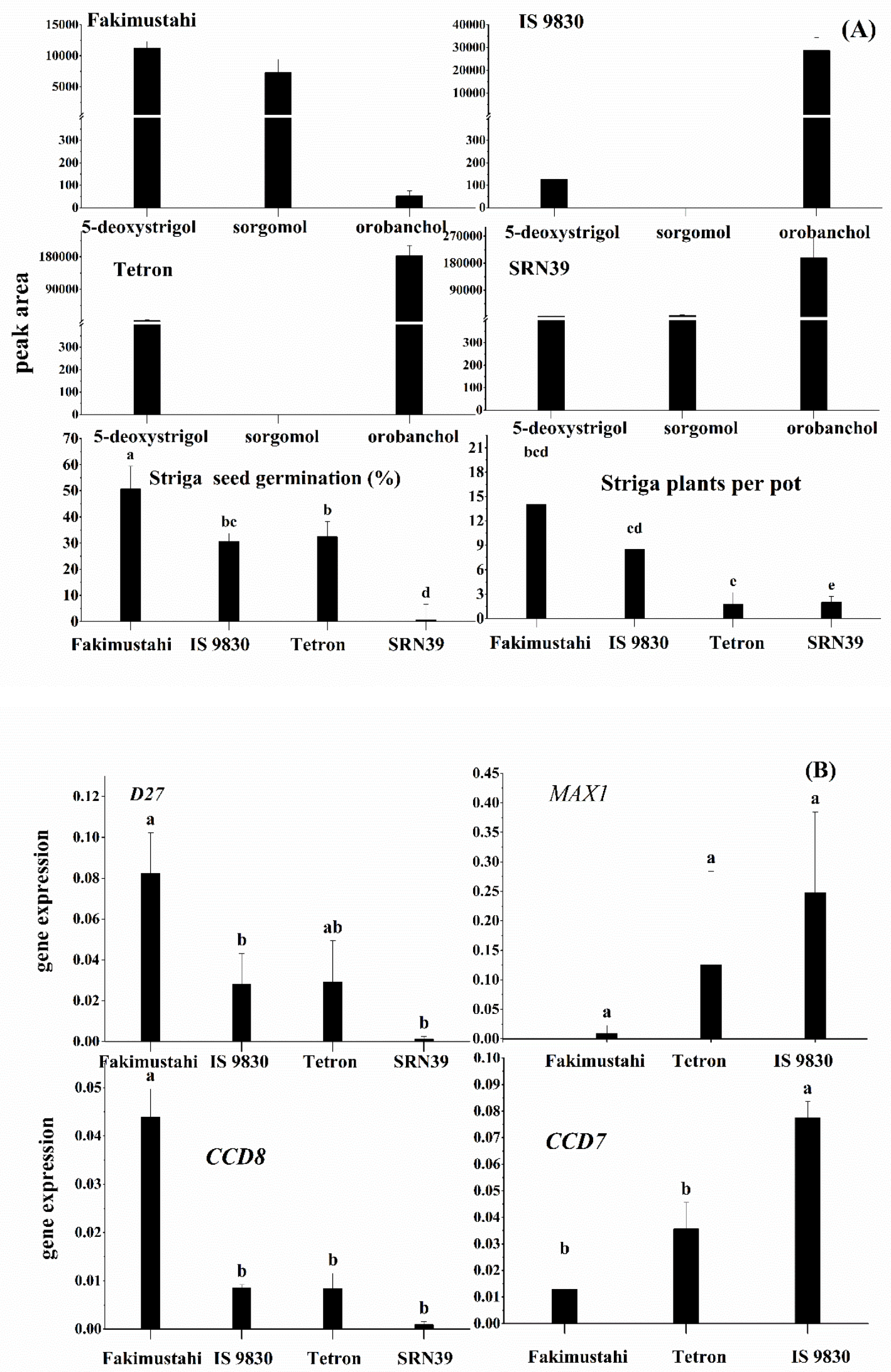

Sorghum genotype 
Fig. 7. (A) Amounts of the strigolactones 5-deoxystrigol, sorgomol, and orobanchol in the root exudates of one Striga-susceptible (Fakimustahi) and three Striga-resistant (IS9830, Tetron, and SRN39) sorghum genotypes used to analyse gene expression, as well as the germination of preconditioned Striga seeds induced by the root exudates of these genotypes, and Striga emergence (plants per pot) in the pot experiment. Data are the means \pm SE of peak area, germination (\%), and total number of emerged Striga plants at harvest $(n=4)$. (B) Relative expression of $D 27, C C D 8, C C D 7$, and MAX1 in the roots of the same four genotypes. Data are means $\pm \mathrm{SE}(n=3)$. The significance of a treatment effect was determined by one-way ANOVA. Different letters above the bars indicate significant differences after Duncan's pairwise comparison $(P<0.05)$.

\section{Markers for germination-stimulating activity in sorghum}

In an attempt to understand the genetics underlying the differences in strigolactone profiles and Striga resistance found in the present study, 12 sorghum genotypes were genotyped. Primers were used corresponding to the marker SB3344, recently reported for the lgs QTL for the low germination stimulant-based resistance of genotype SRN39 (Satish et al., 2012). PCR using these primers generated a polymorphic band pattern discriminating the resistant low-stimulant genotype SRN39 and the susceptible highstimulant genotype Shanqui Red, which lacks the resistance allele (Satish et al., 2012) (Fig. 8A). The size of the PCR-amplified bands ranged from 170 to $190 \mathrm{bp}$. For the genotypes Tetron, IS9830, and Framida, a similar haplotype pattern to that of SRN39 was observed (Fig. 8B); the same was observed for Hakika (data not shown). For the genotypes Wadfahel, Mogud, Wadbaco, and Hariri, a haplotype pattern similar to that of Shanqui Red was observed (Fig. 8B). Two bands were amplified for the genotype Korokollow, while for the genotypes Fakimustahi and Tabat an intermediate-sized band was amplified (Fig. 8B). 

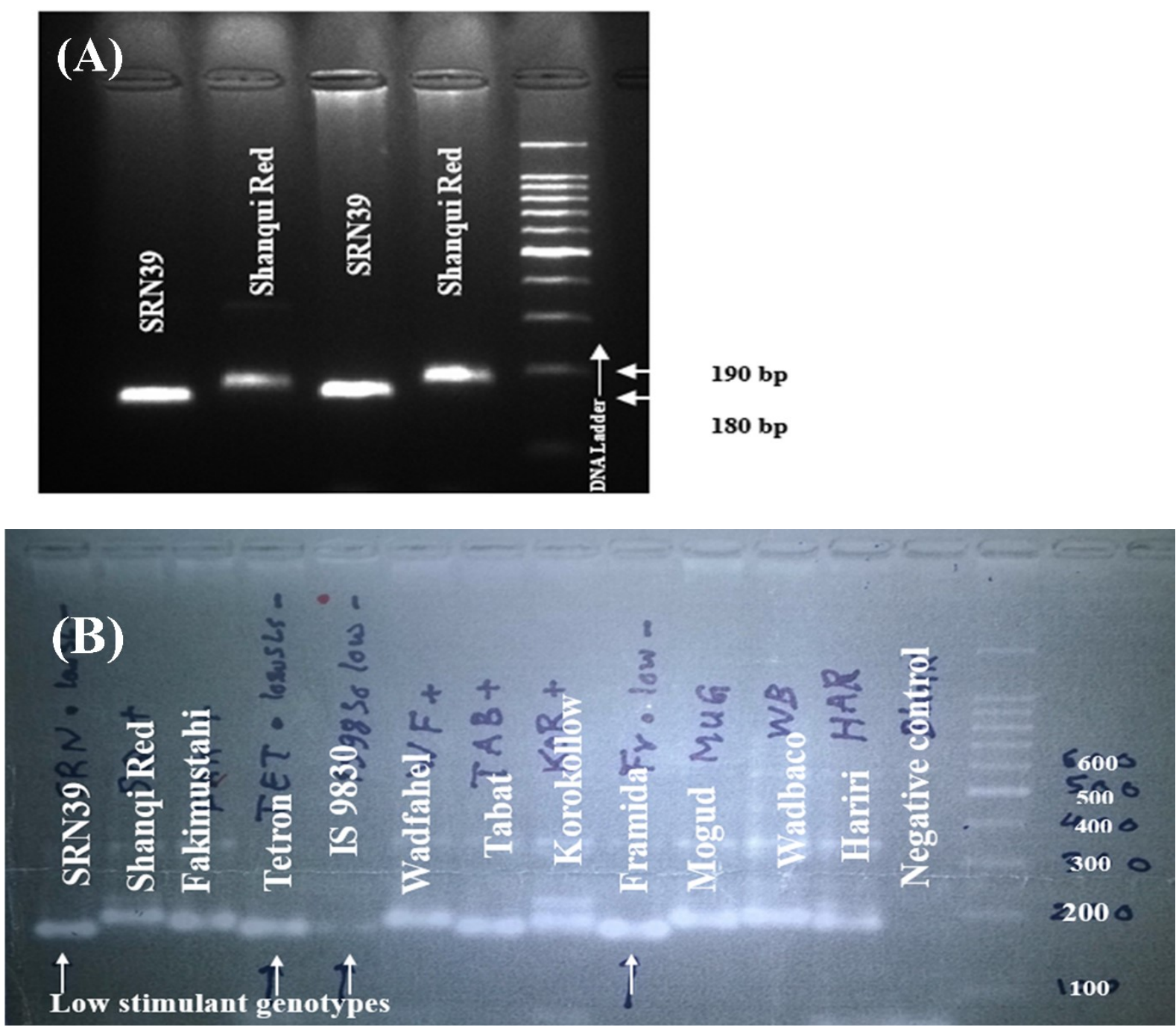

Fig. 8. (A) PCR products obtained with a primer pair for the marker SB3344 and two reference genotypes of sorghum resistant (SRN39) and susceptible (Shanqui Red) to Striga. The fragment sizes for the reference genotypes are $\sim 170$ bp for SRN39 and $\sim 190$ bp for Shanqui Red. (B) PCR products obtained with the marker SB3344 primer pair for 10 further sorghum genotypes. Arrows indicate the amplified fragment of the low-stimulant genotypes. 


\section{Discussion}

Our results reveal that, irrespective of morphological group, geographical location, climatic adaptation, and field reaction to Striga, all sorghum genotypes release 5deoxystrigol, sorgomol, and/or orobanchol as major strigolactones (Supplementary Table S8). In addition, low concentrations of strigol, sorgolactone, ent-2'-epiorobanchol, and ent-2'-epi-5-deoxystrigol were detected in the root exudates of some genotypes. The results also reveal significant genotypic variation in the total concentrations of strigolactones and their relative amounts (Supplementary Table S8; Fig. 2). The production of mixtures of strigolactones and the variation in their amount and composition in sorghum root exudates are in line with several previous reports (Awad et al., 2006; Yoneyama et al., 2010; Jamil et al., 2013). Despite the reports on the identification and characterization of several strigolactones, ent-2'-epi-5deoxystrigol and ent-2'-epi-orobanchol have never been reported before in root exudates or extracts of sorghum. In rice, high levels of ent-2'-epi-5-deoxystrigol were reported in the root exudates of some cultivars (IAC 165, IAC 1246, and Gangweondo; Jamil et al., 2012); whereas ent-2'-epi-orobanchol was reported and characterized in tobacco root exudates as a potent germination stimulant for Phelipanche ramosa L. seeds (Xie et al., 2007). Furthermore, the present study, in line with a previous report (Mohemed et al., 2016), unequivocally confirmed the production of orobanchol by a range of sorghum genotypes, including IS15401 and the Striga-resistant genotypes Framida, Hakika, SRN39, Tetron, and IS9830. Orobanchol was first characterized in red clover by Yokota et al. (1998) and subsequently reported by Jamil et al. (2012) in rice.

In the germination bioassay, 5-deoxystrigol, sorgomol, sorgolactone, and strigol exhibited relatively high germination-inducing activity in Striga seeds compared with the other strigolactones, consistent with previous reports (Hauck et al., 1992; Yasuda et al., 2003; Xie et al., 2008; Cardoso et al., 2014a). Likewise, we showed that at a concentration of $0.02 \mu \mathrm{M}$ ent-2'-epi-5-deoxystrigol, ent-2'-epi-strigol, or GR24 displayed a moderate yet potent activity ( $>30 \%$ germination), while orobanchol showed negligible activity $(<10 \%$ germination). The low germination-inducing activity of orobanchol in Striga is consistent with previous reports on the low sensitivity of Striga 
(sorghum strain) to the orobanchol-type strigolactones (Nomura et al., 2013; Cardoso et al., 2014a). The low sensitivity of Striga to orobanchol suggests that selection for high orobanchol producers may be an effective strategy to obtain sorghum genotypes with good arbuscular mycorrhizal fungi recruitment, but lower Striga germinationinducing activity. It is noteworthy that orobanchol is an effective inducer of mycorrhizal hyphal branching (Akiyama et al., 2010). Indeed, high-orobanchol-producing genotypes were less infected by Striga in a pot experiment (Fig. 6B). Interestingly, we also found a correlation between the level of Striga infection and the Striga emergence rate (Supplementary Table S12). Early emergence of Striga may reflect a highly compatible host-parasite interaction, while late emergence suggests the presence of physical and/or physiological barriers delaying early infection and/or hindering subsequent development (Arnaud et al., 1999; Gurney et al., 1999; Haussmann et al., 2000; Van Ast et al., 2000). Delaying the time of first infection not only influences Striga parasitism and reproduction, but also strongly reduces its damaging effects on host plants (Van Ast and Bastiaans, 2006) (Frost et al., 1997). Whether orobanchol plays a role in the creation of this physical or physiological barrier, or is simply causing lower germination and therefore delayed attachment, remains unclear.

The genotypes used in the present study can be classified into three groups with respect to the timing of Striga emergence (Fig. 5D; Supplementary Table S9). The first group includes Fakimustahi, Feterita Geshaish, Zahrat Elgadambalia, and Tabat, in which Striga emergence was early (15-18 DAS) (Fig. 5A). The second group consists of Arfa Gadamak, Botana, Framida, Korokollow, Mogud, Naten, N13, Najad, Tokarawe, and Wadbaco, in which Striga emergence was moderately early (23-27 DAS), and a third group comprises Hariri, IS9830, SRN39, Tetron, and Wad Ahmed, in which Striga emergence was late (31-36 DAS) (Fig. 5A). These results, which are consistent with a report by Timko and Scholes (2013), suggest that the first group has the least mechanical and/or physiological barriers that delay ingress of the parasite and/or reduce its growth rate, while in the latter two groups constitutive and/or induced barriers may delay the onset of parasitism and/or the growth rate of the parasite (Fig. 5A).

It is noteworthy that all genotypes that showed late Striga emergence possess a strigolactone blend low in 5-deoxystrgol or sorgomol and rich in the less active 
stimulant orobanchol. Furthermore, the results revealed that differences in the proportions of 5-deoxystrigol, sorgomol, and orobanchol in root exudates of different sorghum genotypes influenced their germination-inducing activity and Striga infection (Figs. 3, 4, 6B). The genotypes Feterita Geshaish, Gadam Elhamam and Tabat, whose root exudates contain a high proportion of 5-deoxystrigol and a low proportion of orobanchol, were associated with high Striga germination and displayed high infection levels in pots (Fig. 5). Similarly, the genotypes Fakimustahi and Hariri, the root exudates of which contain a high proportion of sorgomol and a low proportion of orobanchol, were associated with high in vitro germination and high Striga infection. Conversely, the genotypes SRN39, IS9830, Hakika, and Wadbaco, whose the root exudates contain a high proportion of orobanchol and a low proportion of 5deoxystrigol, were associated with low Striga germination and displayed low levels of infection by the parasite. These findings, which are in agreement with previous reports that showed a significant negative relationship between orobanchol, in vitro germination, and field infection by the parasite (Vasudeva Rao, 1984; Mohemed et al., 2016; Gobena et al., 2017), are further substantiated by correlation analysis, which showed the existence of a significant negative association between orobanchol and in vitro Striga germination and Striga emergence in pots (Table 1). The notable negative correlation between orobanchol and 5-deoxystrigol (Fig. 6B) suggests a negative biosynthetic correlation between the two strigolactones, resulting in low production of the strong Striga germination stimulant 5-deoxystrigol when there is a high level of orobanchol. However, a direct inhibitory effect of orobanchol on Striga germination cannot be excluded without further investigation.

The present study further revealed that high stimulant production and in vitro germination are not solely responsible for high infection by the parasite in pot experiments. The genotypes N13 and Tabat have high proportions of 5-deoxystrigol (Supplementary Table S8; Fig. 2) and induced high in vitro germination of Striga (Fig. 3), but N13 displayed far less infection by the parasite than Tabat in the pot experiment (Fig. 5). The differential response in N13 could possibly be attributed to mechanical resistance, as reported by Grenier et al. (2007) and Mbuvi et al. (2017). In addition, high proportions of sorgomol in some genotypes, such as Wadfahel, did not necessarily 
result in higher germination-stimulating activity, although it was associated with a higher Striga infection level in the pot experiment (Fig. 5B). The discrepancy between the results of in vitro germination assays and performance in the pot experiment of some genotypes may be attributable to inherent differences between the two assay methods. Root exudates may vary in composition with the species, cultivar, growth stage, and conditions of growth. Hence, in soils the seeds of the parasite may be exposed to root exudates with a constantly changing composition of strigolactones and other signalling chemicals which may be synergistic or antagonistic to the action of strigolactones (Yoneyama et al., 2009). Nevertheless, there is a good correlation between in vitro germination induced by root exudates and Striga infection in the pot experiment (Fig. 6B). Our findings suggest that, in the absence of other Striga resistance mechanisms, high strigolactone production with a high proportion of 5-deoxystrigol and sorgomol together with low orobanchol in the root exudates results in high susceptibility to Striga. These findings confirm the positive relationship between the level of 5-deoxystrigol in the root exudates of genotypes grown in a greenhouse with Striga infection of the same genotypes in the field previously reported by Mohemed et al. (2016). On the other hand, high proportions of orobanchol in the root exudates enhanced resistance to the parasite. The PCA bi-plot (Fig. 6B) clearly shows that low Striga infection in the pot experiment (in genotypes SRN39, Tetron, IS9830, Framida, Mogud, and Wadbaco) is associated with high proportions of orobanchol, and conversely, susceptibility to the parasite is clearly associated with high proportions of 5-deoxystrigol.

The fact that most of the genotypes we investigated contained mostly the same strigolactones suggests that strigolactone biosynthesis in sorghum is highly conserved. Nevertheless, selection pressure or selection by breeders for Striga resistance seems to have resulted in preferential selection of genotypes that produce strigolactones with low Striga germination-inducing activity, that is, orobanchol (Gobena et al., 2017). The tendency of Striga-resistant sorghum genotypes-previously described as lowgermination-stimulant producers - to produce higher levels of orobanchol shows that it is not the flux through the strigolactone pathway that was affected by selection for Striga resistance in these genotypes, but the flux towards orobanchol, a strigolactone with opposite stereochemistry to 5-deoxystrigol. 
It is therefore also not unexpected that there were no large differences in the expression of the strigolactone biosynthetic genes, all of which catalyse the core strigolactone biosynthetic pathway. Nevertheless, the expression of D27 and CCD8 in root tissues of the susceptible sorghum genotype Fakimustahi was 3- and 4-fold higher, respectively, than in the resistant genotypes IS9830 and Tetron (Fig. 7B). In contrast, expression of $C C D 7$ was higher in the resistant genotype IS9830 than in the susceptible genotype Fakimustahi (Fig. 7B). In rice, Zhang et al. (2014) showed that the MAXI homologs Os900 and Os 1400 catalyse the conversion of carlactone to ent-2'-epi-5-deoxystrigol (deoxyorobanchol) and of ent-2'-epi-5-deoxystrigol (deoxyorobanchol) to orobanchol, respectively. The catalytic function of the four sorghum MAX1s has not been elucidated yet, and there were no significant differences in the expression of one of these MAX1 homologs in the sorghum lines investigated in this study. Accordingly, it is difficult to find a direct correlation between gene expression at this one time point and the levels of the strigolactones in the root exudates; more genotypes need to be studied to pinpoint the roles of these and other genes in the changes in strigolactone biosynthesis in the resistant genotypes.

Genotypes IS9830, Framida, Hakika, and Tetron showed the same amplification of the marker SB3344 as that of the resistant genotype SRN39, confirming the results of Satish et al. (2012). Hakika (also known as P9405) is in fact derived from SRN39 (Mbwaga et al., 2007). The presence of the SB3344 marker coincided with high relative proportions of orobanchol in the root exudates of Tetron (59\%) and IS9830 (66\%), but the root exudates of Framida and Hakika showed less high relative proportions of orobanchol (32\% and $37 \%$, respectively; Fig. 2). It is noteworthy that, based on peak area, IS9830 produced far less 5-deoxystrigol than Tetron and SRN39, and Tetron and IS9830 produced far less sorgomol than SRN39 (Fig. 7).

In essence, the results show that in sorghum genotypes SRN39, IS9830, Framida, Tetron, and Hakika the low-stimulant trait is associated with the same marker (SB3344), and the low-stimulant allele for these genotypes might therefore be identical. However, in the genotypes Wadfahel, Mogud and Wadbaco which are susceptible to the parasite despite the relatively low germination-inducing activity of their root exudates (Figs. 3, 7, 6B), the SRN39 low germination stimulant (lgs) linked marker was not amplified. 
Instead, amplification resulted in a fragment pattern similar to that of the high-stimulant, Striga-susceptible Chinese sorghum genotype Shanqui Red, suggesting that these genotypes lack the resistance allele at the $\lg s$ locus. Genotypes Tabat and Fakimustahi, which produce high levels of 5-deoxystrigol, do not show the expected Shanqui Redtype fragment pattern (Fig. 8B). This may be caused by the possession of different alleles at the $\operatorname{lgs}$ locus in these genotypes or recombination between the marker amplified by the primers and the LGS allele. Korokollow also does not show the typical fragment pattern of either SRN39 or Shanqui Red (Fig. 8B), but this genotype has a quite atypical strigolactone profile (Fig. 2), suggesting the absence of the standard lgs locus.

Our results confirm previous reports that low 5-deoxystrigol and high orobanchol production are associated with low germination traits and low infection by the Striga parasite, and that delayed emergence of the parasite reduces its damaging effects on the host (Gobena et al., 2017; Mohemed et al., 2016). Moreover, the results suggest the involvement of genes other than lgs in controlling the low germination traits in some of the genotypes investigated. These genes could provide additional sources for the introgression of low germination traits from donors other than SRN39 into new preferred sorghum varieties, such as Feteritas, using marker-assisted selection and backcrossing.

\section{Acknowledgments}

The authors acknowledge funding by the International Cooperation in Higher Education (NUFFIC), The Netherlands Fellowship Programmes (NFP) and the Wageningen sandwich fellowship (to NEM), the Netherlands Organization for Scientific Research (to HJB, VICI grant 865.06.002 and equipment grant 834.08.001), the European Research Council (ERC Advanced grant 670211 CHEMCOMRHIZO to HJB) and the Bill and Melinda Gates Foundation (Promise to HJB). The authors would like to thank Dr. Altahir Ibrahim Mohamed, (Plant Genetic Resource Unit), Agricultural Research Corporation, Wad Medani, Sudan for providing sorghum genotypes seeds and their characterization. We would like to thank Prof. Binne Zwanenburg, Prof. Koichi 
Yoneyama and Prof. Tadao Asami for providing strigolactone standards and Dr. Patrick

Rich for helpful comments on the manuscript.

\section{References}

Akiyama K, Ogasawara S, Ito S, Hayashi H. 2010. Structural requirements of strigolactones for hyphal branching in AM fungi. Plant \& Cell Physiology 51, 1104-1117.

Arnaud MC, Véronési C, Thalouarn P. 1999. Physiology and histology of resistance to Striga hermonthica in Sorghum bicolor var. Framida. Australian Journal of Plant Physiology 26, 63-70.

Awad AA, Sato D, Kusumoto D, Kamioka H, Takeuchi Y, Yoneyama K. 2006. Characterization of strigolactones, germination stimulants for the root parasitic plants Striga and Orobanche, produced by maize, millet and sorghum. Plant Growth Regulation, 48, 221-227.

Babiker AGT. 2002. Striga control in Sudan: an integrated approach. In: Leslie JF, ed. Sorghum and millets diseases. Ames: Iowa State Press, 159-163.

Badu-Apraku B, Yalloub CG, Oyekunle M. 2013. Genetic gains from selection for high grain yield and Striga resistance in early maturing maize cultivars of three breeding periods under Striga-infested and Striga-free environments. Field Crop Research 147, 54-67.

Bebawi F. 1981. Intraspecific physiological variants of Striga hermonthica. Experimental Agriculture 17, 419-423.

Bouwmeester HJ, Matusova R, Zhongkui S, Beale MH. 2003. Secondary metabolite signalling in hostparasitic plant interactions. Current Opinion in Plant Biology 6, 358-364.

Cardoso C, Charnikhova T, Jamil M, Delaux PM, Verstappen F, Amini M, Lauressergues D, Ruyter-Spira C, Bouwmeester H. 2014. Differential activity of Striga hermonthica seed germination stimulants and Gigaspora rosea hyphal branching factors in rice and their contribution to underground communication. Plos One 9, e104201.

Cardoso C, Ruyter-Spira C, Bouwmeester HJ. 2011. Strigolactones and root infestation by plant-parasitic Striga, Orobanche and Phelipanche spp. Plant Science 180, 414-420.

Cardoso C, Zhang Y, Jamil M, et al. 2014b. Natural variation of rice strigolactone biosynthesis is associated with the deletion of two MAX1 orthologs. Proceedings of the National Academy of Sciences, USA $111,2379-2384$.

Chang M, Netzly DH, Butler LG, Lynn DG. 1986. Chemical regulation of distance. Characterization of the first natural host germination stimulant for Striga asiatica. Journal of the American Chemical Society $108,7858-7860$.

Ejeta G. 2005. Integrating biotechnology, breeding, and agronomy in the control of the parasitic weed Striga spp in sorghum. In: Tuberosa R, Phillips RL, Gale M, eds. In the wake of the double helix: from the green revolution to the gene revolution. Bologna: Avenue Media, 239-251.

Ejeta G. 2007. The Striga scourge in Africa: a growing pandemic. In: Ejeta G, Gressel J, eds. Integrating new technologies for Striga control: towards ending the witch-hunt. Singapore: World Scientific, $3-16$.

Ejeta G, Butler LG, Babiker AG. 1992. New approaches to the control of Striga. Striga Research at Purdue University, Research Bulletin 991. West Lafayette: Agricultural Experiment Station, Purdue University.

Ejeta G, Mohammed A, Rich P, Melake-berhan A, Housley TL, Hess DE. 2000. Selection for mechanisms of resistance to Striga in sorghum. In: Haussmann BIG, Hess DE, Koyama, ML, Grivet L, Rattunde HFW, Geiger HH, eds. Breeding for Striga resistance in cereals. Proceedings of a workshop held at IITA, Ibadan, Nigeria. Weilkersheim: Margraf Verlag, 29-37. 
Ejeta G, Rich PJ, Mohamed A. 2007. Dissecting a complex trait to simpler components for effective breeding of sorghum with a high level of Striga resistance. In: Ejeta G, Gressel J, eds. Integrating new technologies for Striga control: towards ending the witch-hunt. Singapore: World Scientific, 87-98.

Gobena D, Shimels M, Rich PJ, Ruyter-Spira C, Bouwmeester HJ, Satish Kanuganti S, Mengiste T, Ejeta G. 2017. Mutation in sorghum LOW GERMINATION STIMULANT1 alters strigolactones and causes Striga resistance. Proceedings of the National Academy of Sciences, USA 114, 4471-4476.

Grenier C, Ibrahim Y, Haussmann BI, Kiambi D, Ejeta G. 2007. Marker-assisted selection for Striga resistance in sorghum. In Ejeta G, Gressel J, eds. Integrating New technologies for Striga control: towards ending the witch-hunt. Singapore: World Scientific. 159-172.

Gressel J, Hanafi A, Head G, Marasas W, Obilana AB, Ochanda J, Souissi T, Tzotzos G. 2004. Major heretofore intractable biotic constraints to African food security that may be amenable to novel biotechnological solutions. Crop Protection 23, 661-689.

Guan JC, Koch KE, Suzuki M, Wu S, Latshaw S, Petruff T, Goulet C, Klee HJ, McCarty DR. 2012. Diverse roles of strigolactone signaling in maize architecture and the uncoupling of a branching-specific subnetwork. Plant Physiology 160, 1303-1317.

Gurney AL, Press MC, Scholes JD. 1999. Infection time and density influence the response of sorghum to the parasitic angiosperm Striga hermonthica. New Phytologist 143, 573-580.

Hauck C, Müller S, Schildknecht H. 1992. A germination stimulant for parasitic flowering plants from Sorghum bicolor, a genuine host plant. Journal of Plant Physiology 139, 474-478.

Haussmann BIG, Hess DE, Welz HG, Geiger HH. 2000. Improved methodologies for breeding strigaresistant sorghums. Field Crops Research 66, 195-211.

Hess DE, Ejeta G, Butler LG. 1992. Selecting sorghum genotypes expressing a quantitative biosynthetic trait that confers resistance to Striga. Phytochemistry 31, 493-497.

Jamil M, Charnikhova T, Houshyani B, van Ast A, Bouwmeester HJ. 2012. Genetic variation in strigolactone production and tillering in rice and its effect on Striga hermonthica infection. Planta 235, 473-484.

Jamil M, Van Mourik TA, Charnikhova T, Bouwmeester HJ. 2013. Effect of diammonium phosphate application on strigolactone production and Striga hermonthica infection in three sorghum cultivars. Weed Research 53, 121-130.

Kambal AEL, Musa BM. 1979. Breeding Sorghum cultivars for resistance to Striga. In Technical Progress Report No I. Striga research in the Sudan. Khartoum: Agricultural Research Corporation, Wad Medani, and Faculty of Agriculture, University of Khartoum.

López-Ráez JA, Charnikhova T, Gómez-Roldán V, et al. 2008. Tomato strigolactones are derived from carotenoids and their biosynthesis is promoted by phosphate starvation. New Phytologist 178, 863874.

Matusova R, van Mourik T, Bouwmeester HJ. 2004. Changes in the sensitivity of parasitic weed seeds to germination stimulants. Seed Science Research 14, 335-344.

Mbuvi DA, Masiga CW, Kuria EK, Masanga J, Wamalwa M, Mohamed A, Odeny DA, Hamza N, Timko MP, Runo S. 2017. Novel sources of witchweed (Striga) resistance from wild sorghum accessions. Frontiers in Plant Science 8, 116.

Mbwaga AM, Riches C, Ejeta G. 2007. Integrated Striga management to meet sorghum demand in Tanzania. In: G. Ejeta and J. Gressel, eds. Integrating new technologies for Striga control: towards ending the witch-hunt. Singapore: World Scientific., 253-264.

Mohemed N, Charnikhova T, Bakker EJ, van Ast A, Babiker AG, Bouwmeester HJ. 2016. Evaluation of field resistance to Striga hermonthica (Del.) Benth. in Sorghum bicolor (L.) Moench. The relationship with strigolactones. Pest Management Science 72, 2082-2090.

Nomura S, Nakashima H, Mizutani M, Takikawa H, Sugimoto Y. 2013. Structural requirements of strigolactones for germination induction and inhibition of Striga gesnerioides seeds. Plant Cell Reports 32, 829-838.

Oswald A. 2005. Striga control—technologies and their dissemination. Crop Protection 24, 333-342. 
Parker C. 2009. Observations on the current status of Orobanche and Striga problems worldwide. Pest Management Science 65, 453-459.

Parker CH, Hitchcock AM, Ramaiah, KV. 1977. The germination of Striga species by crop root exudates; techniques for selecting resistant crop cultivars. In Proceedings of the Asian-Pacific Weed Science Society 6th Conference. 67-74.

Priya R, Siva R. 2014. Phylogenetic analysis and evolutionary studies of plant carotenoid cleavage dioxygenase gene. Gene 548, 223-233.

Rich PJ, Grenier C, Ejeta G. 2004. Striga resistance in the wild relatives of sorghum. Crop Science 44, $2221-$ 2229.

Rodenburg J, Bastinaans L. 2011. Host-plant defence against Striga spp.: reconsidering the role of tolerance. Weed Research 51, 438-441.

Rodenburg J, Bastinaans L, Weltzien E, Hess DE. 2005. How can field selection for Striga resistance and tolerance in sorghum be improved? Field Crops Research 93, 34-50.

Satish K, Gutema Z, Grenier C, Rich PJ, Ejeta G. 2012. Molecular tagging and validation of microsatellite markers linked to the low germination stimulant gene (lgs) for Striga resistance in sorghum [Sorghum bicolor (L.) Moench]. Theoretical and Applied Genetics 124, 989-1003.

Schmittgen TD, Livak KJ. 2008. Analyzing real-time PCR data by the comparative C (T) method. Nature Protocols 3, 1101-1108.

Scholes JD, Press MC. 2008. Striga infestation of cereal crops - an unsolved problem in resource limited agriculture. Current Opinion in Plant Biology 11, 180-186.

Siame BA, Weerasuriya Y, Wood K, Ejeta G, Butler LG. 1993. Isolation of strigol, a germination stimulant for Striga asiatica, from host plants. Journal of Agricultural and Food Chemistry 41, 1486-1491.

Spallek T, Mutuku M, Shirasu K. 2013. The genus Striga: a witch profile. Molecular Plant Pathology 14, 861-869.

Taylor JRN. 2003. Overview: importance of sorghum in Africa. In Enhancing Nutritional and Functional Properties for Africa. Proceedings of a Workshop on the Proteins of Sorghum and Millet, April 24 2003, Pretoria, South Africa. Available from: http://www.afripro.org.uk. 1-21.

Tesso TT, Ejeta G. 2011. Integrating multiple control options enhances Striga management and sorghum yield on heavily infested soils. Agronomy Journal 103, 1464.

Timko MP, Scholes JD. 2013. Host reaction to attack by root parasitic plants. In Parasitic Orobanchaceae. Berlin: Springer, 115-141.

Vallabhaneni R, Bradbury LM, Wurtzel ET. 2010. The carotenoid dioxygenase gene family in maize, sorghum, and rice. Archives of Biochemistry and Biophysics 504, 104-111.

Van Ast A, Bastiaans L. 2006. The role of infection time in the differential response of sorghum cultivars to Striga hermonthica infection. Weed Research 46, 264-274.

Van Ast A, Bastiaans L, Kropff MJ. 2000. A comparative study on Striga hermonthica interaction with a sensitive and a tolerant sorghum cultivar. Weed Research 40, 479-493.

Vasudeva Rao MJ. 1984. Patterns of resistance to Striga asiatica in sorghum and millets, with special reference to Asia. In: Ayensu ES, Doggett H, Keynes RD, Marton Lefevre J, Musselman LJ, Parker C, Pickering A, eds. Proceedings of the International Workshop on the Biology and Control of Striga, Dakar. Paris: ICSU Press, 71-92.

Williams C. 1958. The parasitism of witchweed - a review. West African Journal of Biological Chemistry 2, 57-73.

Xie X, Kusumoto D, Takeuchi Y, Yoneyama K, Yamada Y, Yoneyama K. 2007. 2'-epi-orobanchol and solanacol, two unique strigolactones, germination stimulants for root parasitic weeds, produced by tobacco. Journal of Agricultural and Food Chemistry 55, 8067-8072.

Xie X, Yoneyama K, Kusumoto D, Yamada Y, Takeuchi Y, Sugimoto Y, Yoneyama K. 2008. Sorgomol, germination stimulant for root parasitic plants, produced by Sorghum bicolor. Tetrahedron Letters 49, 2066-2068. 
Xie X, Yoneyama K, Yoneyama K. 2010. The strigolactone story. Annual Review of Phytopathology 48, 93-117.

Yasuda N, Sugimoto Y, Kato M, Inanaga S, Yoneyama K. 2003. (+)-Strigol, a witchweed seed germination stimulant, from Menispermum dauricum root culture. Phytochemistry 62, 1115-1119.

Yoder JI, Scholes JD. 2010. Host plant resistance to parasitic weeds; recent progress and bottlenecks. Current Opinion in Plant Biology 13, 478-484.

Yokota T, Sakai H, Okuno K, Yoneyama K, Takeuchi Y. 1998. Alectrol and orobanchol, germination stimulants for Orobanche minor, from its host red clover. Phytochemistry 49, 1967-1973.

Yoneyama K, Arakawa R, Ishimoto K, et al. 2015. Difference in Striga-susceptibility is reflected in strigolactone secretion profile, but not in compatibility and host preference in arbuscular mycorrhizal symbiosis in two maize cultivars. New Phytologist 206, 983-989.

Yoneyama K, Awad AA, Xie X, Yoneyama K, Takeuchi Y. 2010. Strigolactones as germination stimulants for root parasitic plants. Plant \& Cell Physiology 51, 1095-1103.

Yoneyama K, Xie X, Yoneyama K, Takeuchi Y. 2009. Strigolactones: structures and biological activities. Pest Management Science 65, 467-470.

Zhang Y, Van Dijk AD, Scaffidi A, et al. 2014. Rice cytochrome P450 MAX1 homologs catalyze distinct steps in strigolactone biosynthesis. Nature Chemical Biology 10, 1028-1033. 


\section{Supplementary material}

Table S1. Local names, status and collection site of sorghum landraces, improved cultivars, and exotic material used.

\begin{tabular}{|c|c|c|c|}
\hline No & local name & Status & Collection site \\
\hline 1 & Aklamoi & Landrace & East Sudan \\
\hline 2 & Arfa Gadamak & Improved cultivar & East Sudan \\
\hline 3 & Baham & Landrace & Red Sea (Sudan) \\
\hline 4 & Bari & Landrace & South Sudan \\
\hline 5 & Botana & Improved cultivar & East Sudan \\
\hline 6 & Dabar & Landrace & Central Sudan \\
\hline 7 & Dari & Landrace & South Sudan \\
\hline 8 & Debeikri & Landrace & North Sudan \\
\hline 9 & Fakimustahi & Landrace & East Sudan \\
\hline 10 & Feterita Geshaish & Landrace & White Nile \\
\hline 11 & Feterita & Landrace & White Nile (Sudan) \\
\hline 12 & Framida & Exotic & Uganda \\
\hline 13 & Gadam Elhamam & Improved cultivar & East Sudan \\
\hline 14 & Hakika & Exotic & Tanzania \\
\hline 15 & Hariri & Landrace & East Sudan \\
\hline 16 & Hazaztokarwe & Landrace & Red sea (Sudan) \\
\hline 17 & Hemisi & Landrace & North Sudan \\
\hline 18 & IS 15401 & ICRISAT & ICRISAT \\
\hline 19 & IS-9830 & Landrace & Central Sudan \\
\hline 20 & Kolom & Landrace & Blue Nile (Sudan) \\
\hline 21 & Korokollow & Landrace & East Sudan \\
\hline 22 & Markoob & Landrace & West Sudan \\
\hline 23 & Mogud & Landrace & North Sudan \\
\hline 24 & N13 & Landrace & Exotic India \\
\hline 25 & Najad & Landrace & West Sudan \\
\hline 26 & Naten & Landrace & Blue Nile (Sudan) \\
\hline 27 & SRN39 & Synthetic & ICRSAT (Sudan) \\
\hline 28 & Tabat & Improved cultivar & Central Sudan \\
\hline 29 & Tafsagabeid & Landrace & Blue Nile (Sudan) \\
\hline 30 & Tetron & Landrace & West Sudan \\
\hline 31 & Tokarawee & Landrace & Red Sea (Sudan) \\
\hline 32 & Wad Ahmed & Improved cultivar & Central Sudan \\
\hline 33 & Wadbaco & Landrace & East Sudan \\
\hline 34 & Wad Elmardi & Landrace & West Sudan \\
\hline 35 & Wad Fahel & Landrace & White Nile (Sudan) \\
\hline 36 & Zahrat Elgadambalia & Improved cultivar & East Sudan \\
\hline
\end{tabular}

Source: Plant Genetic Resources Unit, Agricultural Research Corporation 
Table S2. D27 (DWARF 27) sorghum orthologous putative gene sequence.

\begin{tabular}{|c|c|c|}
\hline $\begin{array}{l}\text { Gene symbol } \\
\text { Gene description } \\
\text { Gene type }\end{array}$ & $\begin{array}{l}S b D 27 \\
\text { Sb05g022855.1 } \\
\text { protein coding }\end{array}$ & $\begin{array}{l}\text { Gen. Bank ID } \\
8062677 \\
\text { Ref sequence: } \\
\text { X_M002449722.1 }\end{array}$ \\
\hline \multicolumn{3}{|l|}{$>\mathrm{SbD} 27: \mathrm{Sb} 05 \mathrm{~g} 0$} \\
\hline $\begin{array}{l}\text { ATGGAGGTCG } \\
\text { CATGGTCGTCA } \\
\text { CCTACACGAG } \\
\text { GTGGTGGTGG } \\
\text { GGAGAGAACA } \\
\text { CAAGCTGGCC } \\
\text { AAAGGACGGC } \\
\text { TGATCAGCAG } \\
\text { ACAATGGCAA } \\
\text { TTTGAATATAA } \\
\text { CCTTCACCACC } \\
\text { TGATGGAAGG } \\
\text { CAACTGTGTCC } \\
\text { TTCACTTGGCA }\end{array}$ & $\begin{array}{l}\text { AAGCGCCTCGC } \\
\text { JGCGCCACCAG } \\
\text { TGGCGCCGCCC } \\
\text { GGTACCTGTCC } \\
\text { AAGGCCTGATA } \\
\text { AGACTGTGGCC } \\
\text { TAAACACAAGC } \\
\text { AAGATAATGA' } \\
\text { 'TCTTCCCTTGG } \\
\text { AGAAGAATGTC } \\
\text { [GTGCACGAAC } \\
\text { TGTCTACATGT }\end{array}$ & $\begin{array}{l}\text { ATTGGTGTCCTACCGG } \\
\text { ACGAGGCAGAGCTACA } \\
\text { JGCGAGGCCGCAGGAG } \\
\text { ACCAACAACAACAAA } \\
\text { CACGACAGTTGGTTCGA } \\
\text { CTGGGATGAAGAACGG } \\
\text { ГCCGCGCTGTTCAGGGT } \\
\text { TCCCCAGCTACATCCTC } \\
\text { AGAAAGTAGGTTGTTC } \\
\text { ГCGAGAGTACTTTGCCG } \\
\text { TCAGGGAATCCGAAGT } \\
\text { AGATTTCTGGAAAGCAC } \\
\text { AGAAGTTCATCCAAGA } \\
\text { GAGCTGTGAGATGATC }\end{array}$ \\
\hline
\end{tabular}


Table S3. CCD7 (CAROTENOID CLEAVAGE DIOXYGENASE 7) orthologous putative gene sequence.

\begin{tabular}{|c|c|c|}
\hline Gene symbol & CCD7 & \\
\hline Gene description & $\begin{array}{l}\text { Carotenoid cleavage dioxygenase } \\
\mathrm{Sb} 06 \mathrm{~g} 024560\end{array}$ & 8055963 \\
\hline Gene type & protein coding & $\begin{array}{l}\text { Ref sequence: } \\
\text { X_M002446857.1 }\end{array}$ \\
\hline Location: & \multicolumn{2}{|l|}{ chromosome: 6} \\
\hline \\
\hline \multirow{2}{*}{\multicolumn{3}{|c|}{ ATGCACGCCGCCGTGCACCACCACCACCCTGGCCACCGCGCACCGCCGCCTCGCCGCTGCT }} \\
\hline & & \\
\hline \\
\hline \multicolumn{3}{|c|}{ ACCTCCTGTTCCGGTCGCAGCGCGCCGAGTGCCGCGACCCCGTCGCGCTCCGCGTCACCGA } \\
\hline \multicolumn{3}{|c|}{ GGGCGCGATCCCGGCGGACTTCCCGTCGGGCACCTACTACCTCGCCGGTCCGGGGATGTTC } \\
\hline \multicolumn{3}{|c|}{ ACCGACGACCACGGGTCCACCGTGCACCCGCTCGACGGCCACGGCTACCTCCGCTCGTTCC } \\
\hline \multicolumn{3}{|c|}{ GCTTCGGCTCCGACGGCGCGCCGGCGCGCTACTCCGCGCGGTACGTGGAGACGGCGGCGA } \\
\hline \multicolumn{3}{|c|}{ AGCGGGAGGAGCACGACGCGCCGCGCTCGTCGTGGCGGTTCACGCACCGGGGCCCC } \\
\hline \\
\hline \multicolumn{3}{|c|}{ ACCAGCGTGCTGCGCTGGGGCGGCCGCGTGCTCTGCCTCTGGGAAGGCGGCGAGCCGTAC } \\
\hline \multicolumn{3}{|c|}{ GAGCTGGACCCGCGGACGCTGGAGACCATCGGCCCGTTCGACATCCTCGGCCGCCTCGCC } \\
\hline \multicolumn{3}{|c|}{ GGGCGGCGAAGCGGCACGAGACGACGACAGCAGCGAGGCTGCGCGTCTCGGGCGCCG } \\
\hline \multicolumn{3}{|c|}{ SGCCGTGGCTGCAGGAGGCAGGGATCGACGTGGCCGCGCGCCTGCTGCGACCGGTCCT } \\
\hline \multicolumn{3}{|c|}{ CGGTGGTGTCTTCAGCATGCCGGCCAAGCGGCTGCTCGCGCACTACAAGATCGACCAGGA } \\
\hline \multicolumn{3}{|c|}{ GAGGAACCGGCTGCTCATGGTGGCCTGCAACGCCGAGGACATGCTCCTCCCGCGCTCCAA } \\
\hline \multicolumn{3}{|c|}{ CTTCACTTTCTACGAGTTCGACGCCGACTTCGCGCTGGTGCAGACGCGGGAGTTTGTCTTG } \\
\hline \multicolumn{3}{|c|}{ CCGGACCACCTGATGATCCACGACTGGACCTTCACGGACAGCCACTACGTCСТCCTCGGCA } \\
\hline \multicolumn{3}{|c|}{ ACAGAATCAGGCTGGACATTCCAGGTTCGCTGCTGGCGCTCACGGGCACTCACCCAATGA } \\
\hline \multicolumn{3}{|c|}{ TCGCGGCCCTCGCCGTGGACCCGAGCCGGCAGTCCACGCCCGTCTACCTGCTGCCGCGCTC } \\
\hline \multicolumn{3}{|c|}{ CCCGGAGGCCGAGGCCCCCGGCGCGACTGGAGCGTGCCCATCGAGGCGCCATCGCAGAT } \\
\hline \multicolumn{3}{|c|}{ GTGGTCCATGCACGTCGGCAACGCCTTCGAGGAGCGCAAC } \\
\hline \multicolumn{3}{|c|}{ TCAGCTCCACATGTCCGGCTGCTCTTACCAGTGGTTCAACTTCCACAGGATGTTTGGTTAC } \\
\hline \multicolumn{3}{|c|}{ AATTGGCAGAACAAGAAGCTGGACCCGTCCTTCATGAACATAGCCAAGGGCAGGGAATG } \\
\hline \multicolumn{3}{|c|}{ GCTACCTCGTCTTGTACAGGTGTCCATCGACCTCGACAAGAGAGGAACGTGCCGAGGATG } \\
\hline \multicolumn{3}{|c|}{ CTCCGTCCGGAGATTGTCCGACCAGTGGACCAGGCCGGCGGACTTCCCGGCGATCAACCC } \\
\hline \multicolumn{3}{|c|}{ AGGCTTCGCCAACCGGAGGAACCGGTTCATCTACGCCGGCGGCGCCTCCGGTTCACGCAG } \\
\hline \multicolumn{3}{|c|}{ ATTCTTGCCGTACTTCCCCTTCGACAGCGTCGTCAAGGTAGACGTCGCTGATGGATCAGCG } \\
\hline \multirow{2}{*}{\multicolumn{3}{|c|}{ 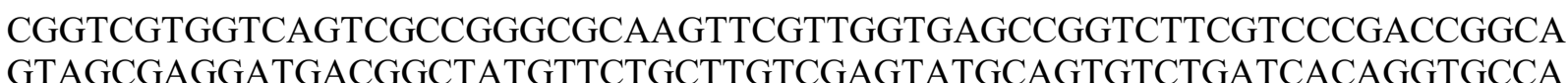 }} \\
\hline \multirow{2}{*}{\multicolumn{3}{|c|}{ GTAGCGAGGATGACGGCTATGTTCTGCTTGTCGAGTA }} \\
\hline & & \\
\hline & & \\
\hline
\end{tabular}


Table S4. CCD8 (CAROTENOID CLEAVAGE DIOXYGENASE 8) orthologous putative gene sequence.

\begin{tabular}{lll}
\hline Gene symbol & $C C D 8$ & Gen. Bank ID \\
\hline Gene description & Carotenoid cleavage dioxygenase & 8062181 \\
& Sb03g034400.1 & \\
Gene type & protein coding & Ref sequence: \\
& & X_M002458432 \\
Location: & chromosome: 3 & \\
\hline
\end{tabular}

>SbCCD8: Sb03g034400.1

ACCAGTACACACCACACTCTCGGCTAGCTAGCTAGCGAGCTACTACTACTGGACCTCTCCT TTCCTTATTAGCTCCAAATTAAAAGCCCCGCCCCTGGTACTACAACCCTAGCAAGAGCATT TTGCCATGTCTCCCACTATGGCTTCGTCGCTCTGCGTGTTTGCAGCGATGTCTGGCGCCACC GGCAGGCCGTCGTCGACCGGTGGCTCGGTGGTGCCGCCGGGCCGTCTGTCCAGTACTGCA CAGGGGACCAAGGGAAAGAGGGCCGTGGTGCAGCCTCTCGCGGCTAGCGTCGTGACGGA CACGCCGACGCCGACGCCGGCCATAGCTCCGGCGGCCCCGCCAGCTCGGCCAGTCGTCGA CGCCCCGCGCCGCCGTGGGGGCCGCGGCACCGGCGAGCACGCGGCGTGGAAGAGCGTCC GGCAGGAGAGGTGGGAGGGGGCGCTGGAGCTGGAGGGAGAGCTGCCGCTCTGGCTGGAT GGCACGTACCTGAGGAACGGCCCGGGCCTGTGGAACCTGGGCGACTACGGCTTCCGGCAC CTGTTCGACGGCTACGCGACGCTGGTCCGCGTCTCGTTCCGCAACGGGCACGCGGTGGGC GCGCACCGGCAGATCGAGTCGGAGGCGTACAAGGCGGCGCGCGCGAACGGCAAGGTGTG CTACCGCGAGTTCTCGGAGGTGCCCAAGGCGGACAGCTTCCTCTCCCACGTGGGCCAGCTC GCCACCCTCTTCTCGGGCTCCTCGCTCACCGACAACTCCAACACGGGCGTGGTCAGGCTCG GCGACGGCCGCGTGCTGTGCCTGACGGAGACCATCAAGGGCTCCATCGTGGTCGACCCGG ACACGCTGGACACCCTCGGCAAGTTCGAGTACACGGACAAGCTGGGCGGCCTCATCCACT CGGCGCACCCCATCGTGACGGACACCGAGTTCTGGACGCTGATCCCGGACCTCATCCGCC CGGGCTACTCGGTGGTGCGGATGGACGCCGGGACCAACGAGCGGCGGTTCGTCGGCAGGG TGGACTGCCGCGGCGGGCCGGCGCCCGGGTGGGTGCACTCGTTCCCCATCACGGACCACT ACGTGGTCGTGCCGGAGATGCCGCTCCGGTACTGCGCCAGGAACCTCCTCCGCGCGGAGC CCACCCCGCTGTACAAGTTCGAGTGGCACCTCGAGTCCGGCAGCTACATGCACGTCATGTG CAAGGCCAGCGGCAGGGTCGTGGCCAGCGTGGAGGTGCCGCCCTTCGTCACCTTCCACTT CATCAACGCGTACGAGGAGAAGGACGAGGAGGGCCGCGTCACGGCGATCGTCGCCGACT GCTGCGAGCACAACGCCAACACCACCATCCTCGACAAGCTCCGGCTCCAGAACCTCCGCT CTTCCACCGGGCAGGACGTCCTCCCGGACGCCAGGGTGGGCCGGTTCAGGATCCCGCTGG ACGGGAGCCCGTTCGGCGAGCTGGAGTCGGCGCTGGACCCGGACCAGCACGGCCGCGGG ATGGACATGTGCAGCATCAACCCGGCCCACGTCGGCAAGAAGTACCGGTACGCCTACGCC TGCGGCGCCCAGCGGCCGTGCAACTTCCCAAACACCCTCACCAAGATCGACCTGGTGGAG AAGACGGCCAAGAACTGGTACGAGGAGGGCGCCGTGCCGTCAGAGCCCTTCTTCGTGCCG CGCCCGGGCGCCGTGGAGGAGGACGACGGCGTTGCGATTTCGATGGTGAGCGCCAAGGAC GGATCGGCGTACGCGCTAGTGCTGGACGCCAAGACGTTCCAGGAGATCGCGCGGGCCAAG TTCCCGTACGCGATGCCCTACGGTTTGCACTGCTGCTGGGTGCCTAGGACCACCTCAGACG CGTAG 
Table S5. SbMAX1 orthologous putative gene sequence.

\begin{tabular}{lll}
\hline Gene symbol & SbOrtholog $(M A X I)$ & Gen. Bank ID \\
Gene description & Sb03g032220 & SbOrtholog $($ MAXI) \\
cytochrome P450 & 8068068 \\
Gene type & protein coding & Ref sequence: \\
Location: & chromosome: 3 & XM_002456168.1 \\
\hline
\end{tabular}

>SbMAX1: Sb03g032220

ATGGAGATGGGCACGGTTCTGGGTGCCATGGAGGAGTACACCTTCACCTTCCTGGCCATG GCCGTGGGGTTCCTAGTGCTTGTGTATCTGTACGAGCCGTACTGGAAGGTGCGCCACGTGC CCGGGCCCGTGCCGCTGCCGCTCATCGGCCACCTCCACCTGCTGGCGAAGCACGGCCCAG ACGTCTTCCCTGTGCTCGCCAAGAAGCACGGGCCTATCTTCAGATTTCACGTGGGAAGGCA GCCATTGATCATCGTGGCCGACGCAGAGCTCTGCAAGGAGGTGGGCATCAAGAAGTTCAA GAGCATGCCTAACCGGAGCTTGCCTTCGCCCATCGCCAATTCTCCCATACATCGGAAAGGC CTCTTCGCTACAAGGGATTCGAGGTGGTCGGCGATGCGAAACGTTATCGTCTCGATCTACC AGCCGTCGCACCTCGCCGGGCTGATGCCGACCATGGAGTCGTGCATCGAGCGCGCTGCGA CGACGAACCTCGGCGATGGCGAGGAGGTCGTCTTCTCCAAGCTGGCGCTGAGCCTCGCCA CCGACATCATCGGGCAAGCGGCGTTCGGCACAGACTTTGGCCTCTCGGGAAAGCCGGTGG TGCCTGACGATGACATGAAGGGTGTCGACGTCGTCGTCGGAGATGCAGCGAAGGCGAAGG CGTCATCATCGGAATTCATAAACATGCACATCCACTCGACCACGTCGCTCAAGATGGACCT GTCGGGGTCGCTCTCCACCATCGTTGGCGCGCTCGTGCCCTTCCTGCAGAATCCGCTGCGG CAGGTGCTCTTGAGGGTCCCCGGCTCCGCCGACCGGGAGATCAACCGTGTGAACGGCGAG CTCCGTAGGATGGTGGACGGCATCGTCGCCGCCCGCGCAGCGGAGAGGGAGCGTGCGCCG GCGGCGACAGCAGCACAGCAGCACAAGGACTTCCTCTCCGTGGTGCTGGCGGCGAGGGAG AGCGACGCGTCCACGCGGGAGCTGCTCTCGCCGGACTACTTGAGCGCGTTGACCTACGAG CACCTCATCGCTGGGCCGGCGACCGCGGCGTTCACGCTGTCCTCCGTGGTCTACCTCGTCG CCAAGCACCCGGAAGTGGAGGAGAAGCTGCTTAGGGAGATGGACGCGTTCGGCCCTCGCG GCAGTGTGCCCACGGCGGATGATCTTCAGACCAAGTTCCCCTACCTCGATCAGGTGGTGA AGGAGTCGATGAGGCTGTTCATGGTGTCGCCATTGGTGGCGCGAGAGACCTCCGAGCGAG TGGAGATCGGTGGCTATGTGCTTCCAAAGGGCGCATGGGTGTGGATGGCACCAGGGGTCC TCGCCAAGGACGCCCACAACTTCCCGGACCCGGAGCTGTTCCGACCAGAGCGGTTCGACC CAGCCGGCGACGAGCAAAAGAAGCGTCACCCATACGCGTTCATCCCTTTTGGAATCGGCC CCAGGGTTTGCATCGGCCAGAAGTTCGCCATCCAGGAGATCAAGCTCGCGATAATCCACC TCTATCAGCACTATGTGTTCCGGCATTCTCCCAGCATGGAGTCCCCTCTAGAGTTTCAGTTT GGGATTGTGGTCAACTTCAAGCATGGTGTCAAGCTTCACGTCATCAAGAGGCATGTGGAA AATAATTAA 
Table S6. Primers used for quantitative RT-PCR.

\begin{tabular}{ll}
\hline Predicted locus/ Gene symbol & Primer pair \\
\hline SbD27 & F_TGTTCAGGGTTGATCAGCAG \\
Sb05g022855.1 & R_CGAGGGTGGCATCATTATCT \\
SbCCD8 & F_GAGTACTTTGCCGCCTTCAC \\
Sb03g034400.1 & R_ACTTCTGGCATGGGATTTTG \\
SbCCD7 & F_ACGCAGATTCTTGCCGTACT \\
Sb06g024560 & R_CAAGCAGAACATAGCCGTCA \\
SbMAX1 & F_GTGGAGATCGGTGGCTATGT \\
Sb03g032220 & R_AAAAGGGATGAACGCGTATG \\
SbActin 1 & F_ACATTGCCCTGGACTACGAC \\
Actin & R_TGATGACCTGTCCATCAGGA \\
\hline
\end{tabular}

Table S7. Primers used for quantitative PCR for marker analysis.

\begin{tabular}{ll}
\hline Sequence Name & Sequence \\
\hline SB3344-F & GCC TCT GCC TCT TGG AAT CAG TTA \\
SB3344-R & AAG AAA GGG AGT AAC CGG ATG AGC \\
\hline
\end{tabular}


Table S8. Genotypic variation in strigolactone production in sorghum.

\begin{tabular}{|c|c|c|c|c|}
\hline No & Genotype & 5-deoxystrigol* & sorgomol* & orobanchol* $^{*}$ \\
\hline 1 & Aklamoi & 114 abcdefghi & 2.65 defghji & 0.28 efghji \\
\hline 2 & Arfa Gadamak & 95.9 abcdefgh & $0.00 \mathrm{~m}$ & $0.08 \mathrm{jk}$ \\
\hline 3 & Baham & 118 abcdef & $0.02 \mathrm{jklm}$ & $0.02 \mathrm{cdef}$ \\
\hline 4 & Bari & 92.2 fhgji & $3129 \mathrm{a}$ & 2.57 cde \\
\hline 5 & Botana & 32.6 abcdefhgi & $0.09 \mathrm{klm}$ & $1.92 \mathrm{cdef}$ \\
\hline 6 & Dabar & 21.1 abcdefhgi & 0.08 hjikl & 0.11 efghji \\
\hline 7 & Dari & 125 abcdegh & $1362 \mathrm{a}$ & 2.14 cdef \\
\hline 8 & Debeikri & $602 \mathrm{ab}$ & 0.18 hgjik & 0.44 cdefgh \\
\hline 9 & Fakimustahi & 261 abcdef & $270 a b$ & $0.07 \mathrm{jk}$ \\
\hline 10 & Feterita & 427 abcd & 0.09 defgh & 0.09 defghi \\
\hline 11 & Feterita Geshaish & $262 \mathrm{abcd}$ & 0.80 efhgjki & 0.57 defghi \\
\hline 12 & Framida & 189.15 abcdefhgi & $0.75 \mathrm{jikl}$ & $7.56 \mathrm{bc}$ \\
\hline 13 & Gadam Elhamam & 172 abcdef & 0.69 defhgi & 0.38 cdefgh \\
\hline 14 & Hakika & $0.21 \mathrm{kl}$ & 1.32 cdefgh & $1.36 \mathrm{cdef}$ \\
\hline 15 & Hariri & 2.03 kijl & $9.45 \mathrm{~cd}$ & 0.05 cdefe \\
\hline 16 & Hazaztokarwe & $361 \mathrm{abcd}$ & 0.22 hgjik & 0.11 efghji \\
\hline 17 & Hemisi & 2832 abcdefhg & 26.6 cdefg & $4.80 \mathrm{cdef}$ \\
\hline 18 & IS 15401 & 7.64 fhgji & $2.10 \mathrm{cdefgh}$ & 0.26 efghji \\
\hline 19 & IS-9830 & 0.121 & $0.00 \mathrm{~m}$ & $28.7 \mathrm{~cd}$ \\
\hline 20 & Kolom & $579 \mathrm{a}$ & 0.13 hgjik & 0.22 defghi \\
\hline 21 & Korokollow & 14.6 cdehfgji & $0.00 \mathrm{~m}$ & 0.23 defghi \\
\hline 22 & Markoob & 179 abcdef & 0.96 fhgjik & 0.18 ghijk \\
\hline 23 & Mogud & 8.49 efhigj & $0.01 \mathrm{~lm}$ & 0.14 defghi \\
\hline 24 & N13 & 817 a & $3.45 \mathrm{cdef}$ & 0.59 cdefghi \\
\hline 25 & Najad & 524 abcdef & $6.58 \mathrm{~cd}$ & $1.97 \mathrm{cdef}$ \\
\hline 26 & Naten & 10.1 defhgji & $19.8 \mathrm{bc}$ & 0.26 defghi \\
\hline 27 & SRN39 & 3.05 hjki & $5.71 \mathrm{cde}$ & $197 \mathrm{ab}$ \\
\hline 28 & Tabat & $558 \mathrm{abc}$ & 2.93 cdefg & 0.58 cdefg \\
\hline 29 & Tafsagabeid & 328 abcde & 0.13 hgjikl & 0.04 hjik \\
\hline 30 & Tetron & $2.62 \mathrm{jkl}$ & $0.00 \mathrm{~m}$ & $183 \mathrm{a}$ \\
\hline 31 & Tokarawe & 192 fhgji & 4.48 defhgi & 0.41 efghji \\
\hline 32 & Wad Ahmed & 244 abcdef & $1.73 \mathrm{~m}$ & $2.56 \mathrm{cdef}$ \\
\hline 33 & Wad Elmardi & 187 abcdef & $1335 \mathrm{a}$ & $2.28 \mathrm{cdef}$ \\
\hline 34 & Wadbaco & 3.18 hgijk & $1.68 \mathrm{cdefg}$ & 0.48 cdefg \\
\hline 35 & Wad Fahel & 111 abcdefg & $18.6 \mathrm{bc}$ & 0.11 efghji \\
\hline 36 & Zahrat Elgadambalia & 86.2 abcdefgh & $4.43 \mathrm{cde}$ & 0.35 cdefgh \\
\hline
\end{tabular}

*Peak area $\times 10^{3}$ 
Table S9. Emergence rate, expressed as days to the first Striga emergence.

\begin{tabular}{llc}
\hline No. & genotype & Days to first Striga emergence \\
\hline 1 & Aklamoi & $15 \mathrm{i}$ \\
2 & Arfa Gadamak & $24 \mathrm{f}$ \\
3 & Botana & $27 \mathrm{e}$ \\
4 & Fakimustahi & $18 \mathrm{~h}$ \\
5 & Feterita Geshiash & $15 \mathrm{i}$ \\
6 & Framida & $24 \mathrm{f}$ \\
7 & Hariri & $31 \mathrm{~d}$ \\
8 & Gadam Elhamam & $18 \mathrm{~h}$ \\
9 & IS-9830 & $33 \mathrm{c}$ \\
10 & Korokollow & $23 \mathrm{~g}$ \\
11 & Mogud & $24 \mathrm{f}$ \\
12 & Najad & $24 \mathrm{f}$ \\
13 & Naten & $27 \mathrm{e}$ \\
14 & N13 & $24 \mathrm{f}$ \\
15 & SRN39 & $36 \mathrm{a}$ \\
16 & Tabat & $15 \mathrm{i}$ \\
17 & Tetron & $33 \mathrm{c}$ \\
18 & Tokarawe & $24 \mathrm{f}$ \\
19 & Wad Ahmed & $35 \mathrm{~b}$ \\
20 & Wadbaco & $27 \mathrm{e}$ \\
21 & Wad Fahel & $16 \mathrm{i}$ \\
22 & Zahrat Elgadambalia & $18 \mathrm{~h}$ \\
\hline
\end{tabular}


Table S10. Principal components, eigenvalues, loadings, and percentage of total variance explained in principal component analysis.

\begin{tabular}{lrrrrrrr}
\hline \multicolumn{3}{l}{ For data shown in Fig. 7A } \\
\hline \multicolumn{3}{c}{ Total variance (\%) } & \multicolumn{4}{c}{ Eigen vector loading for } \\
PC & \multicolumn{1}{c}{ E $^{*}$} & In & Cum & \multicolumn{1}{c}{ 5-de } & \multicolumn{1}{c}{ sorg } & \multicolumn{1}{c}{ orb } & \multicolumn{1}{c}{ Germ } \\
\hline 1 & 1.85 & 47.29 & 47.29 & 0.61 & 0.24 & -0.44 & 0.60 \\
2 & 1.09 & 26.84 & 74.13 & 0.15 & 0.82 & 0.52 & -0.11 \\
3 & 0.62 & 15.72 & 89.84 & 0.27 & -0.45 & 0.72 & 0.43 \\
4 & 0.48 & 10.16 & 100.00 & -0.72 & 0.21 & 0.01 & 0.65 \\
\hline
\end{tabular}

For data shown in Fig. 7B

\begin{tabular}{lrrrrrrrr}
\hline & \multicolumn{3}{c}{ Total variance (\%) } & \multicolumn{4}{c}{ Eigen vector loading for } \\
PC & \multicolumn{1}{c}{ E } & In & \multicolumn{1}{l}{ Cum } & \multicolumn{1}{c}{ 5-de } & \multicolumn{1}{c}{ sorg } & \multicolumn{1}{c}{ orb } & \multicolumn{1}{l}{ Striga } & \multicolumn{1}{l}{ Germ } \\
\hline 1 & 2.63 & 52.67 & 52.67 & 0.45 & 0.31 & -0.49 & 0.53 & 0.40 \\
2 & 1.02 & 20.49 & 73.16 & 0.25 & 0.79 & 0.18 & -0.12 & -0.50 \\
3 & 0.74 & 14.86 & 88.03 & 0.59 & -0.05 & 0.51 & -0.36 & 0.49 \\
4 & 0.35 & 7.08 & 95.11 & -0.60 & 0.51 & 0.10 & -0.13 & 0.57 \\
\hline
\end{tabular}

* E, Eigen value; In, Individual; Cum, Cumulative; 5-de, 5-deoxystrigol; sorg, sorgomol; orb, orobanchol; Germ, Germination \%; Striga, Striga plants per pot.

Table S11. Correlation coefficients between strigolactone peak area in the root exudates and expression of biosynthetic genes $D 27$ and $C C D 8$, Striga germination, and Striga emergence.

\begin{tabular}{llll}
\hline & 5 -deoxystrigol & sorgomol & orobanchol \\
\hline D27 & $0.70^{* *}$ & $0.73^{* *}$ & $-0.38 \mathrm{NS}$ \\
CCD8 & $-0.80^{* *}$ & -0.71 & $0.21 \mathrm{NS}$ \\
\hline
\end{tabular}

**indicates significance at $\mathrm{P}<0.05$

Table S12. Pearson correlation between numbers of emerged Striga per pot, total Striga biomass per pot, and emergence rate.

\begin{tabular}{lccc}
\hline Striga number & Striga biomass $(\mathrm{mg})$ & Days to first Striga emergence & Striga number \\
\hline Striga numbers & 1 & $0.43^{*}$ & $-0.53^{*}$ \\
Striga biomass $(\mathrm{mg})$ & 1 & $-0.56^{*}$ \\
Days to first Striga emergence & & & 1 \\
\hline
\end{tabular}




\section{Chapter 3}

\section{Genetic variation in tolerance to Striga infection in sorghum: underlying processes and the relationship with strigolactones.}

Nasreldin M. Ahmed, A. van Ast, A.G.T. Babiker, Harro J. Bouwmeester

Abstract

Background: The root parasitic weed Striga hermonthica (Striga) is a major constraint to cereal production and a threat to food security in sub-Saharan Africa. In the absence of complete resistance to Striga, the capacity of crops to be tolerant to Striga, i.e. to give a reasonable yield even under Striga infection, is a valuable trait. In several landraces of sorghum tolerance has been suggested to play a role in safeguarding yield in Striga infested fields. The mechanisms underlying tolerance to Striga are not yet understood, making selection for this trait difficult. In this study we therefore analysed twenty sorghum genotypes from Sudan for Striga tolerance and measured a number of morphological and physiological parameters to try to further our understanding of tolerance and root exudates Striga germination stimulant content.

Results: Striga infection reduced biomass accumulation in all sorghum genotypes analysed irrespective of Striga infection level but the relative impact varied significantly between the different genotypes suggesting genotype-specific differences in tolerance to the parasite. Striga infection decreased the maximum rate of photosynthesis and carboxylation efficiency and increased the concentration of $\mathrm{CO}_{2}$ in the sub-stomatal cavities and $\mathrm{ABA}$ in the leaves. Sorghum biomass strongly and positively correlated with presence of the germination stimulant orobanchol, photosynthesis parameters but only weakly and inversely with emerged Striga numbers and biomass.

Conclusions: Our experimental setup allowed uncoupling of Striga resistance (infection level) and tolerance (lower effect of Striga on sorghum biomass and photosynthesis parameters). Tolerance identified earlier in the field for some genotypes was confirmed 
under controlled conditions, providing evidence that tolerance is largely genetically determined. Genotypes Hariri, Mogud, Wad Ahmed and Wad Baco delayed Striga emergence time, exhibited low to moderate reduction in total biomass, maximum $\mathrm{CO}_{2}$ assimilation rate, stomatal conductance and photosynthetic carboxylation efficiency despite a high Striga infection and were thus identified as tolerant. Genotypes Aklamoi, Fakimustahi, Feterita Geshaish, Najad and Naten, which demonstrated early Striga emergence, severe reduction in total biomass, $\mathrm{CO} 2$ assimilation rate, stomatal conductance and photosynthetic carboxylation efficiency, were identified as sensitive. We conclude that the genotypes SRN39, IS9830 and Tetron, which exhibited delayed and low Striga emergence, low reduction in biomass, $\mathrm{CO}_{2}$ assimilation rate, stomatal conductance and photosynthetic carboxylation efficiency, exhibit a combination of resistance and tolerance. The knowledge generated here can be used to breed for genotypes with combined resistance and tolerance. Further we suggested here strigolactones content as a novel tool for selection for tolerance.

\section{Introduction}

The witchweed Striga hermonthica [Del.] Benth. (Striga) is one of the most destructive root parasitic flowering plants. The parasite causes huge yield losses in important staple crops including maize (Zea mays [L.]), sorghum (Sorghum bicolor [L.] Moench), and rice (Oryzasativa [L.]) (Parker, 2009). Infestations with Striga in many African countries are severe and have reached epidemic levels especially in marginal lands cultivated by resources limited farmers (Ejeta, 2007). The widespread occurrence of Striga in African countries and the large losses in grain yield and biomass are due to the prolific nature of Striga and the high susceptibility and sensitivity of most crop varieties (Dangi et al., 1989). Most cultural and chemical control interventions that have been suggested so far are deemed either too expensive or impractical. Cereal varieties that are not or less infected by Striga or suffer less from an infection have been proposed as the best solution for resourcelimited farmers (Parker and Riches, 1993). However, efforts to develop immune crop varieties with stable and durable broad spectrum resistance against prevalent Striga spp. 
have hardly been successful. The lack of immunity and durability of resistance is impounded by the high level of genetic variation in the Striga populations, high seed production rates and rapid build-up of persistent seed banks (Rodenburg et al., 2006). Resistance to Striga refers to a crop trait that reduces or prevents infection and reproduction, while tolerance refers to a crop trait that results in lower or minimum yield losses upon infection (Rodenburg and Bastiaans, 2011). Tolerance does not preclude parasite development, seed production or a buildup of the seed bank that perpetuates future infestations. Resistance often is neither complete nor durable and loss of resistance, particularly under high level of infestation, has been reported (Parker and Riches, 1993; Ejeta, Mohammed et al. 2000; Rodenburg and Batiaans, 2011). Therefore, resistance (low infection levels) and tolerance (minimal Striga-induced host damage) are complementary traits and employment of genotypes endowed by both is an interesting breeding objective (Kim, 1991; Haussmann et al 2000; Kling et al., 2002). As Striga is native to Africa, many local cereals, including sorghum and millet, have shown some degree of tolerance and resistance to the parasite (Mboob, 1989; Rodenburg et al., 2008). However, the possibility of transferring these traits into elite high yielding varieties was only initiated at ICRISAT in 1983 (Dangi et al., 1989). Based on host plant damage scores, yield loss or relative yield loss, under Striga infestation, some varieties of sorghum and maize have been identified as tolerant (Efron, 1993; Adetimirin et al., 2000; Gurney et al., 2002; Oswald and Ransom, 2004). However, the complexity of the infection process and the effect of a multitude of interacting variables including the environment, the host plant and the Striga population and seed bank size on this process often make differentiation between resistance and tolerance difficult (Rodenburg et al., 2015). This notion is corroborated by the conflicting reports on resistance and tolerance of the sorghum genotypes CMDT39, Framida, IS9830 and SRN39 (Rodenburg, 2005). The difficulty of screening for tolerance under field conditions has been extensively discussed by Rodenburg and Bastiaans (2011). Rodenburg et al. (2006) indicated that field selection is impractical and requires creation of Striga-free control treatments. Moreover, yield loss of certain varieties could be due to both differences in host resistance level as well as differences in host tolerance level. Selection based only 
on host plant damage and yield loss under Striga infestation will not account for differences in the level of host resistance. Precise quantification of host tolerance is further complicated by the non-linear relation between Striga infection level and yield loss (Gurney et al. (1999).

Sorghum as one of the major Striga hosts, produces root exudates that contain germination stimulants, called strigolactones (SLs), which trigger parasitic plant seed germination. Several studies have shown that a low production of germination stimulants endows a sorghum genotype with resistance to Striga in the field (Bouwmeester et al., 2003; Ejeta et al., 2002). Sorghum and other plant species secrete SLs because they induce hyphal branching of arbuscular mycorrhizal (AM) fungi, which is beneficial for the host (Akiyama et al., 2005). This symbiosis not only helps the plants to acquire nutrient and water from a larger soil volume and hence protects plants against abiotic stress but also protects the plant from biotic stresses (Smith et al., 2010). In rice, tolerance to Striga was mapped to the same region harboring a strong QTL involved in strigolactone production associated with higher Striga infection but late emergence. This observation suggests that SLs might reduce Striga infection efficiency and/or increase host tolerance. If this could be substantiated this could result in better tools for the selection for tolerance. In this study we therefore investigated the relationship between strigolactones produced in the root exudates of 20 sorghum genotypes and their role in tolerance to the Striga infection.

To further improve the understanding of Striga tolerance, we also studied the morphological and physiological response of the host to Striga infection. Several studies have shown that sorghum often responds to Striga infection with remarkable reduction in morphological and phenological development as well as biomass and yield. The symptoms of a Striga infection include stunting and wilting as a consequence of the withdrawal by Striga of host carbon assimilates, water, mineral nutrients and amino acids and its elusive, pathological effect (Stewart et al., 1991; Gurney et al., 1995; Press and Graves, 1995). In addition, perturbations in the hormonal balance of the host occur, such as an increase in the level of abscisic acid (ABA) and decrease in indole-3-acetic acid (IAA), cytokinins and gibberellins (GA53, GA19, GA20) (Drennan and El Hiweris, 1979; Clark et al., 1994; 
Spallek et al., 2013). Striga infection is renowned for the significant reduction in photosynthesis in the host and that has been reported as the most important growth reducing factor (Frost et al., 1997a). Graves et al. (1989) estimated that $80 \%$ of the decrease in host growth rate can be attributed to the impact of Striga on its photosynthesis. Reduction of the photosynthetic rate in infected plants is likely due to stomatal closure, as a consequence of the increased $\mathrm{ABA}$ production in the host and/or metabolic changes other than $\mathrm{ABA}$ production or stomata closure. For example changes in the activity of ribulose-1,5bisphosphate carboxylase/oxygenase (Rubisco) in $\mathrm{C} 3$ plants or phosphoenol pyruvate carboxylase (PEPC) in C4 plants (Frost et al., 1997a; Press et al., 1999; Watling et al., 2001). However, there is variation between sorghum genotypes in the extent to which these responses occur - possibly reflecting differences in tolerance - and it has been suggested that this could offer a screening tool for host tolerance to Striga (Rodenburg et al., 2008). The objective of the present study was to investigate variation in host tolerance to Striga in twenty sorghum genotypes from Sudan and to characterize strigolactones, physiological parameters (photosynthesis, stomatal conductance, and ABA) - to find a physiological marker showing a correlation with tolerance.

\section{Materials and Methods}

\section{Plant material and growth conditions}

Seeds of twenty sorghum genotypes, including improved cultivars, local landraces and exotics were provided by the gene bank of the Agricultural Research Corporation (ARC), Wad Medani Sudan. The genotypes, collected based on assumed differences in resistance and tolerance to Striga infection, were Tabat, Fakimustahi, Naten and Feterita Geshaish representing susceptible and sensitive genotypes; SRN39, Tetron and IS9830, representing resistant genotypes; and Mogud, Hariri, Wad Ahmed and Wad Baco supposed to represent tolerant genotypes. Local names and identities of the twenty sorghum genotypes are listed together with details of their agronomic traits in Table 1. Striga (Striga hermonthica) seeds were collected in 2010 from sorghum grown at the ARC Abu Naama Research Station, Sudan. 
Table 1 Local names, reported reactions to Striga and agronomic traits of the sorghum genotypes used in the present study

\begin{tabular}{|c|c|c|c|c|c|}
\hline No & Local Name & $\begin{array}{l}\text { Resistance/ } \\
\text { Susceptible }\end{array}$ & $\begin{array}{l}\text { Tolerance } \\
\text { /Sensitive }\end{array}$ & Group & Key traits \\
\hline 1 & Aklamoi & $?$ & $?$ & $?$ & Landrace \\
\hline 2 & Arfa Gadamak & S & $\mathrm{T}$ & Feterita & Farmer preference \\
\hline 3 & Botana & S & $\mathrm{T}$ & Feterita & Farmer preference \\
\hline 4 & Fakimustahi & S & $\mathrm{Sn}$ & Milo & Bird resistant, late \\
\hline 5 & Feterita Geshaish & S & $\mathrm{Sn}$ & Feterita & Landrace \\
\hline 6 & Hariri & S & $\mathrm{T}$ & & Landrace \\
\hline 7 & IS9830 & $\mathrm{R}$ & S & Feterita & Striga resistant \\
\hline 8 & Korokollow & $\mathrm{S}$ & $\mathrm{T}$ & Feterita & Farmer preference, \\
\hline 9 & Mogud & $\mathrm{TS}$ & $\mathrm{T}$ & Mogud & Striga tolerant** \\
\hline 10 & N13 & $\mathrm{R}$ & $\mathrm{T}$ & $?$ & Striga resistant \\
\hline 11 & Najad & TS & $\mathrm{T}$ & Heigiri & Striga tolerant \\
\hline 12 & Naten & $?$ & $?$ & $?$ & Landrace \\
\hline 13 & SRN39 & $\mathrm{R}$ & $\mathrm{T}$ & Synthetic & Striga resistant \\
\hline 14 & Tabat & S & $\mathrm{Sn}$ & Milo & Farmer preference, cv \\
\hline 15 & Tetron & $\mathrm{R}$ & $\mathrm{T}$ & Feterita & Striga resistant \\
\hline 16 & Tokarawe & $?$ & $?$ & $?$ & Landrace \\
\hline 17 & Wad Ahmed & S & $\mathrm{T}$ & Feterita & Farmer preference* \\
\hline 18 & Wad Baco & $\mathrm{TS}$ & $\mathrm{T}$ & Milo & Striga tolerant \\
\hline 19 & Wadfahel & $\mathrm{S}$ & $\mathrm{Sn}$ & Feterita & Farmer preference, late \\
\hline 20 & Zahrat Elgadambalia & $\mathrm{S}$ & $?$ & Feterita & Farmer preference, cv \\
\hline
\end{tabular}




\section{Analysis of root exudates for strigolactone content}

For collection of root exudates, germinated sorghum seeds of each genotype were planted in a $3 \mathrm{~L}$ plastic pot filled with $1.5 \mathrm{~L}$ sand. After one week, plants were thinned to 5 plants per pot. Half strength modified Hoagland's nutrient solution with corresponding phosphorus concentration was applied to each pot (500 mL at $48 \mathrm{~h}$ intervals). The plants were allowed to grow under controlled conditions in a climate room with artificial lighting at $450 \mu \mathrm{mol} / \mathrm{m}^{2} / \mathrm{second}\left[\left(28^{\circ} \mathrm{C}(\mathrm{D}) 10 \mathrm{~h}\right.\right.$ and $\left.25^{\circ} \mathrm{C}(\mathrm{N}) 14 \mathrm{~h}\right]$ and $70 \%$ relative humidity for four weeks. In the 5th week, phosphorus deficiency was created in each pot to increase strigolactone production (Lopez-Raez et al., 2008). Three litres phosphorus deficient nutrient solution (half strength modified Hoagland's nutrient solution minus phosphate) were added to each pot and allowed to drain freely through holes at the bottom of each pot. The plants were kept under P deficiency for one week prior to flushing each pot with $3 \mathrm{~L}$ of phosphorus deficient nutrient solution to remove accumulated strigolactones. The plants were allowed to grow for, $48 \mathrm{~h}$ where root exudates were collected in $1 \mathrm{~L}$ plastic bottle by passing $3 \mathrm{~L}$ nutrient solution without phosphate through each pot. Strigolactones, from each pot, captured on an SPE C18 column $(500 \mathrm{mg}$ per $3 \mathrm{~mL})$ were eluted with $6 \mathrm{~mL}$ acetone. For further purification acetone was evaporated under vacuum at $25^{\circ} \mathrm{C}$ using a rotary evaporator. The residues dissolved, each, in $4 \mathrm{~mL}$ hexane, were, loaded, each, on a pre-equilibrated Silica gel Grace Pure SPE $(200 \mathrm{mg} / 3 \mathrm{~mL})$ column and eluted with $2 \mathrm{~mL}$ hexane:ethyl acetate (1:9). The solvents were evaporated and the residue was dissolved in $200 \mu \mathrm{L}$ of $25 \%$ aqueous acetonitrile and filtered through Minisart SRP4 $0.45 \mu \mathrm{m}$ filters (Sartorius, Germany) prior to LC-MS/MS analysis.

\section{Strigolactone analysis}

The strigolactones, 5-deoxystrigol, sorgomol and orobanchol were identified and quantified using ultra performance liquid chromatography tandem mass spectrometry (UPLC-MS/MS) as previously described by Lopez-Raez et al., 2008. The samples were analysed on a Waters Xevo triple quadrupole tandem mass spectrometer (Waters, Milford, MA, USA) equipped with electrospray ionization source and coupled to an Acquity UPLC 
system (Waters, USA). Multiple reaction monitoring (MRM) was used for quantification of strigolactones in sorghum root exudates. Data acquisition and analysis were performed using Mass Lynx 4.1 (TargetLynx) software (Waters).

\section{Assessment of tolerance to Striga}

The sorghum genotypes were grown in pots in a glasshouse at Wageningen University, The Netherland, to examine genotypic tolerance responses to Striga. Plants were grown in $18 \mathrm{~L}$ pots $(1 \times \mathrm{w} \times \mathrm{h}=0.25 \mathrm{~m} \times 0.25 \mathrm{~m} \times 0.30 \mathrm{~m})$ containing a mixture of sand and arable soil, collected from the top layer $(0-0.25 \mathrm{~m})$ of an arable field near Wageningen. Striga seeds $(8 \mathrm{mg})$ were added and mixed through this soil in each pot. Five seeds of each sorghum genotype were sown in the middle of the pot and the seedlings were thinned to one10days after emergence (DAS).The plants were grown in a temperature-controlled glasshouse set at $28^{\circ} \mathrm{C}(12 \mathrm{~h}) 25^{\circ} \mathrm{C}(12 \mathrm{~h})$ and $50-60 \%$ relative humidity. Supplemental light was provided by high-pressure sodium lamps (400 W SON-T, Agro Philips lamps), which automatically switched on during day time when photosynthetically active radiation (PAR) outside the greenhouse dropped below $910 \mu \mathrm{mol}$ photons $\mathrm{m}^{-2} \mathrm{~s}^{-1}$. Black screens were used to reduce the day length to $12 \mathrm{~h}$ throughout the entire growing period. Half strength modified Hoagland's nutrient solution was applied in the first week $(250 \mathrm{ml}$ at 48 $\mathrm{h}$ intervals). For the remainder of the experimental period nutrient solution with only $20 \%$ $\mathrm{P}$ was applied to stimulate strigolactone exudation $(250 \mathrm{~mL}$ to each pot at $48 \mathrm{~h}$ intervals).

Striga counts were performed every 3 days, up to 60 DAS and subsequently at weekly intervals. At maturity (DAS70-115) sorghum plants were harvested and severed into leaves, stems and roots. Leaf area was determined with a leaf area meter (Li-3100 area meter; Li-cor, Lincoln, NB, USA). Plant organs including stems, leaves, panicles and roots, oven dried at $70^{\circ} \mathrm{C}$ for $72 \mathrm{~h}$, wereweighed. Striga plants, attached to sorghum roots were removed, counted, washed, similarly dried and weighed. A Striga tolerance index (TI) was calculated as $\mathrm{I} / \mathrm{C}^{*} 100$, where $\mathrm{I}$ is the value of the total biomass produced under Striga infection, and $\mathrm{C}$ represents the total biomass produced under Striga free conditions. 


\section{ABA analysis and photosynthetic response}

The genotypes, Hariri, Wad Ahmed, Wad Baco, and Mogud, which seemed to exhibit tolerance, the low stimulant producing, resistant, genotypes SRN39 and Tetron (Kambal, 1979; Bebawi, 1981) and the genotypes Naten, Najad and Aklamoi, which did not seem to exhibit tolerance were selected to study the effect of Striga infection on ABA accumulation and photosynthesis.

ABA analysis. Samples (c0.3-0.5 g each) from young upper leaves form each genotype were collected $52 \mathrm{DAS}$, ground in liquid nitrogen and subsequently transferred to a10-mL glass vial with $2 \mathrm{~mL}$ of cold ethyl acetate containing [2H]6-ABA as internal standard (0.05 nmol. $\mathrm{mL}^{-1}$ ). The vials, vortexed and sonicated for $10 \mathrm{~min}$ in a Branson 3510 ultrasonic bath (Branson Ultrasonics, Danbury, CT, USA), were centrifuged for $10 \mathrm{~min}$ at $2500 \mathrm{~g}$. The organic phase was transferred to a 4-mL glass vial and the pellets were re-extracted without sonication, with another $2 \mathrm{~mL}$ of ethyl acetate containing no internal standard. The combined ethyl acetate extract was dried in a vacuum centrifuge for $1 \mathrm{~h}$. The residues were dissolved in $50 \mu \mathrm{L}$ methanol and vortexed after which $3 \mathrm{~mL}$ milliQ water were added. After vortexing, the samples were loaded onto a SPE C18 column (500 mg) column and subsequently eluted with $1 \mathrm{~mL}$ of acetone. The acetone was evaporated and the residue dissolved into $200 \mu \mathrm{L}$ of acetonitrile: water $\left(\mathrm{ACN}: \mathrm{H}_{2} \mathrm{O}, 10: 90\right)$, vortexed and filtered into $400 \mu \mathrm{L}$ (LC/MS/MS) vials prior to injection for ABA analysis. ABA was determined by comparing retention time and mass transitions with those of an ABA standard using a Waters Xevo tandem mass spectrometer (Waters, Milford, MA, USA) equipped with an electrospray ionization (ESI) source and coupled to an Acquity UPLC system (Waters) as described previously (Lopez-Raez et al., 2010).

Photosynthetic responses. Maximum $\mathrm{CO}_{2}$ assimilation rate $\left(\mathrm{A} \max ; \mu \mathrm{mol} \mathrm{CO}_{2} \mathrm{~m}^{-2} \mathrm{~s}^{-1}\right)$, stomatal conductance $\left(g s ; \mathrm{mol} \mathrm{m} \mathrm{m}^{-2} \mathrm{~s}^{-1}\right)$, transpiration rate (Evpt; mmol $\mathrm{H}_{2} \mathrm{O} \mathrm{m} \mathrm{m}^{-2} \mathrm{~s}^{-1}$ ), carboxylation efficiency ( $Ф \mathrm{CO} 2 ; \mu \mathrm{mol} \mathrm{CO}_{2} \mathrm{~m}^{-2} \mathrm{~s}^{-1}$ ), sub-stomatal concentration of $\mathrm{CO}_{2}$ $\left(\mathrm{C} i ; \mu \mathrm{mol} \mathrm{CO} \mathrm{mol}^{-1}\right)$ were measured at at $55 \mathrm{DAS}$ between 10.30 and 14.30 , along the length of the youngest fully expanded leaf, on 3 plants (replicates) per genotype. A LI6400XT Portable Photosynthesis System (LI-COR Bioscience, Lincoln, Nebraska USA) 
was used at photosynthetic photon flux density of around $\left(1200 \mu \mathrm{mol} \mathrm{m} \mathrm{m}^{-2} \mathrm{~s}^{-1}\right)$. ФCO2, electron transport rate through PSII (ETR), photochemical quenching (qP) and efficiency of photosystem II (ФPS2) were calculated (Rodenburg, 2008; Manual, L. I. C. O. R., 2005).

\section{Statistical analysis}

Reductions in plant height, leaf area, shoot dry weight, root dry weight and total biomass under Striga infestation, were calculated, for each genotype as the difference between means recorded under Striga infected and Striga-free conditions. The responses of each genotype to Striga infections were subjected to ANOVA using PROC GLM in SAS (SAS Inc., Version 9.1) and the means were separated using Duncan's multiple comparisons test. Sorghum genotypes were considered as fixed effects in the Analysis of variance (ANOVA) of each trait. Pearson correlation coefficients between strigolactones, tolerance index, physiological and morphological traits were generated using IBM SPSS Statistics 20 packages. The Principal Component Analysis (PCA) was conducted on standardized average values obtained from the 20 sorghum genotypes in four replications using RStatistic bioinformatics packages. Principal component analysis was done using mean values of morphological and physiological traits, strigolactone peak area, to estimate the contribution of these traits to tolerance to Striga, visualize distance between genotypes and delineate which strigolactones, morphological or physiological adjustments coincide with tolerance. 


\section{Results}

\section{Striga infection}

The total number of emerged Striga plants per pot differed significantly $(P<0.001)$ among the genotypes (Table 2). Striga emergence was high (20.5-14.3 plants per pot) on Tabat, Najad, Hariri, Framida, Fakimustahi and Mogud, moderately high (10.7-13.6) on Naten, Korokollow, Wad Ahmed, Wad Baco, Zahrat Elgadambalia, Feterita Geshaish, Tokarawe, N13 and Arfa Gadamak, moderate (8.3-9.8) on Botana, IS-9830 and Aklamoi and low (2 and 2.3 plants per pot) on SRN39 and Tetron.

Striga emergence was very early (15-18 DAS) on Aklamoi, Fakimustahi, Feterita Geshaish, Tabat and Zahrat Elgadamblia, moderately early (23-27 DAS) on Arfa Gadamak, Botana, Framida, Korokollow, Mogud, Naten, N13, Najad, Tokarawe, and Wad Baco and late (31-36 DAS) on Hariri, IS-9830, SRN39, Tetron and Wad Ahmed (Table 2). Also the total Striga biomass differed significantly $(P<0.001)$ among genotypes (Table 2$)$. Aklamoi, Arfa Gadamak, Feterita Geshaish, Framida, N13, Najad, Tabat and Wad Baco, supported the highest (250-323 mg per pot) biomass. Botana, Fakimustahi, Hariri, Korokollow, Mogud, Naten, Tokarawe, Wad Ahmed and Zahrat Elgadambalia sustained moderately high (200-230 mg per pot) biomass, while SRN39, IS-9830 and Tetron supported the lowest (52-95 mg per pot) biomass. 
Table 2 Mean emerged Striga plants and total biomass per pot per genotype over the entire growing period \pm standard error $(\mathrm{n}=4)$ for each genotype and days to first Striga emergence. The significance of genotypic differences were determined by a one-way ANOVA. Means within a column followed by a different letter are significantly different according to Duncan's pairwise comparison $(\mathrm{P}<0.05)$.

\begin{tabular}{lllll}
\hline No. & Genotype & $\begin{array}{l}\text { Number of emerged } \\
\text { Striga plants/pot }\end{array}$ & Striga biomass/pot (mg) & DFE \\
\hline 1 & Aklamoi & $8.3 \mathrm{~d}$ & $250 \mathrm{a}$ & $15 \mathrm{i}$ \\
2 & Arfa Gadamak & $10.8 \mathrm{~cd}$ & $250 \mathrm{a}$ & $24 \mathrm{f}$ \\
3 & Botana & $9.8 \mathrm{~d}$ & $200 \mathrm{abc}$ & $27 \mathrm{e}$ \\
4 & Fakimustahi & $17.7 \mathrm{abc}$ & $202.5 \mathrm{abc}$ & $18 \mathrm{~h}$ \\
5 & Feterita Geshiash & $11.0 \mathrm{~cd}$ & $267.5 \mathrm{a}$ & $15 \mathrm{i}$ \\
6 & Framida & $16.8 \mathrm{abc}$ & $275.0 \mathrm{a}$ & $24 \mathrm{f}$ \\
7 & Hariri & $18.3 \mathrm{ab}$ & $227.5 \mathrm{abc}$ & $31 \mathrm{~d}$ \\
8 & IS-9830 & $8.5 \mathrm{~d}$ & $80.0 \mathrm{~d}$ & $33 \mathrm{c}$ \\
9 & Korokollow & $13.3 \mathrm{bcd}$ & $221.25 \mathrm{ab}$ & $23 \mathrm{~g}$ \\
10 & Mogud & $14.3 \mathrm{abcd}$ & $230.0 \mathrm{ab}$ & $24 \mathrm{f}$ \\
11 & Najad & $18.8 \mathrm{ab}$ & $312.5 \mathrm{a}$ & $24 \mathrm{f}$ \\
12 & Naten & $13.6 \mathrm{bcd}$ & $322.5 \mathrm{ab}$ & $27 \mathrm{e}$ \\
13 & N13 & $10.7 \mathrm{~cd}$ & $217.5 \mathrm{a}$ & $24 \mathrm{f}$ \\
14 & SRN39 & $2.0 \mathrm{e}$ & $95.0 \mathrm{~cd}$ & $36 \mathrm{a}$ \\
15 & Tabat & $20.5 \mathrm{a}$ & $315.0 \mathrm{a}$ & $15 \mathrm{i}$ \\
16 & Tetron & $2.3 \mathrm{e}$ & $52.75 \mathrm{~d}$ & $33 \mathrm{c}$ \\
17 & Tokarawe & $11.0 \mathrm{~cd}$ & $205.0 \mathrm{abc}$ & $24 \mathrm{f}$ \\
18 & Wad Ahmed & $13.1 \mathrm{bcd}$ & $220.0 \mathrm{ab}$ & $35 \mathrm{~b}$ \\
19 & Wad Baco & $12.8 \mathrm{bcd}$ & $282.5 \mathrm{a}$ & $27 \mathrm{e}$ \\
20 & Zahrat Elgadambalia & $12.6 \mathrm{bcd}$ & $210 \mathrm{ab}$ & $18 \mathrm{~h}$ \\
\hline
\end{tabular}

*DFE $=$ Days to first Striga emergence

The number of emerged Striga plants showed a positive correlation with Striga biomass $(r=0.43 ; P<0.05)$ and there was a negative correlation between time to first Striga 
emergence and Striga number per pot and total Striga biomass $(r=-0.53$ and -0.56 , respectively; $P<0.05$; Table $\mathrm{S} 1$ ) (Fig. 1).
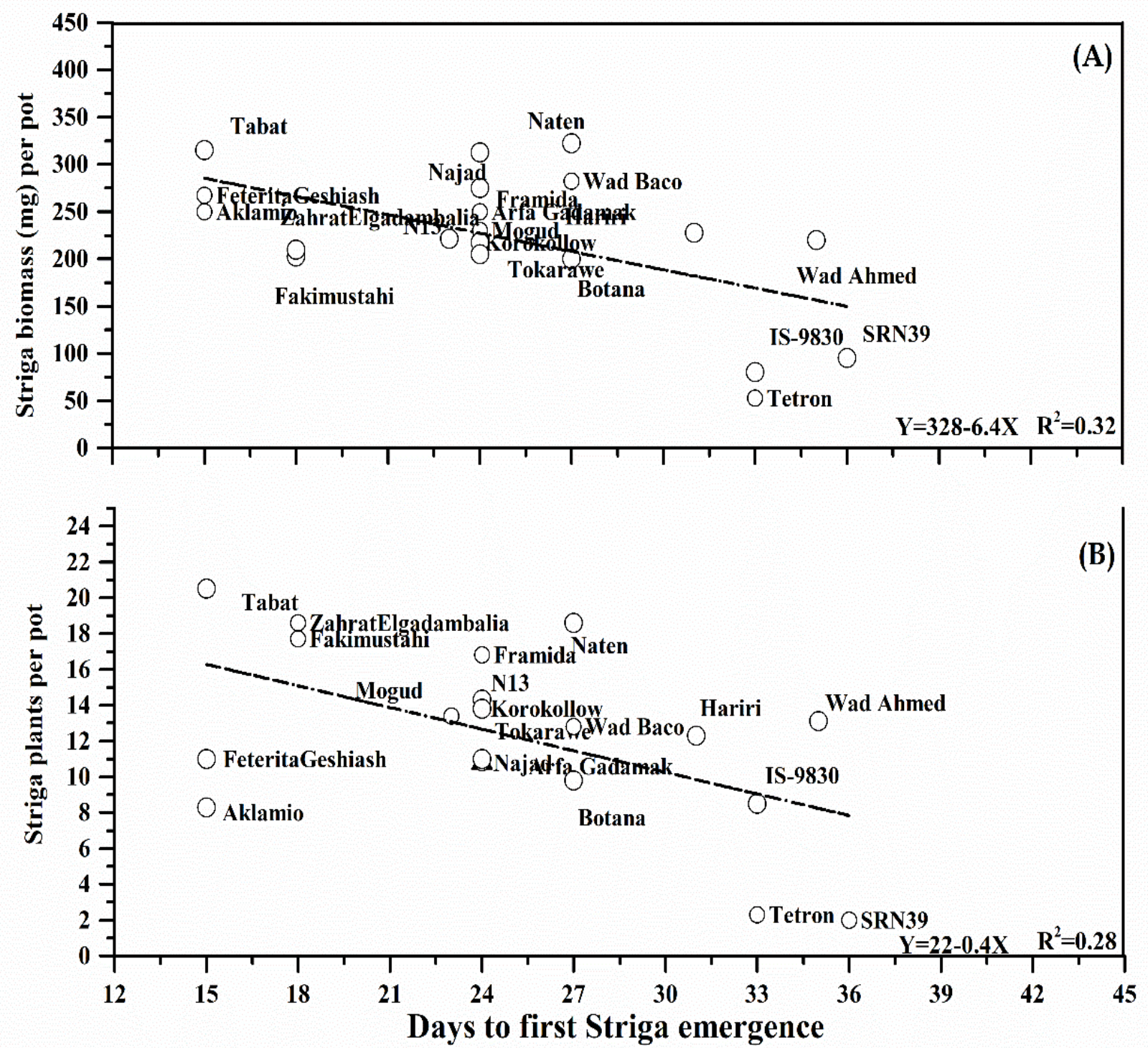

Fig. 1 A) the relationship between Striga biomass (mg) per pot and days to first Striga emergence B) relationship between numbers of emerged Striga plants per pot and days to first Striga emergence. Data presented as means of 4 replicates \pm SE. The line of best fit (Regression analysis) is indicated. 


\section{Morphological and physiological trait analysis}

Changes in morphological and physiological traits in response to Striga infection were analysed to investigate genotype-specific responses to Striga infection (Table S2). There was a significant $(P<0.05)$ negative effect of Striga infection on the morphological traits height, leaf area, and shoot, root and total biomass (Table S2). To visualize the effect of Striga on sorghum morphology a PCA was carried out. Fig. 2 shows the scores (A) and loadings (B) plots of the first two Principal Components. PC1 and PC2 have an Eigenvalue $>1$, account for $69 \%$ and $21 \%$ of the variation, respectively, together explain $90 \%$ of the variation and provide the best visualization of the separation between the genotypes (Table 3A; Fig. 2). Descriptors such as sorghum height, root biomass, shoot biomass and total biomass were the morphological traits with highest positive loadings in PC1 (Table 3A). Their close proximity in the loadings plot shows that they are highly correlated. The genotypes are clustered in two, partially overlapping, clusters representing the control (2) and the infected treatment (1). Genotypes located in quadrant III and IV of the scores plot have low height, and root, shoot and total biomass (Fig. 2A). The genotypes located in quadrant I and II have high height and root, shoot and total biomass, and this is true not only under Striga-free conditions but for some genotypes also under Striga infection, for example, SRN39, Tetron, Hariri and IS9830. For each genotype, the distance in the PCA plot between the infected and control condition is indicative of the impact that Striga has on the morphological parameters. For example Fakimustahi, Feterita Geshaish, Zahrat Elgadambalia and Korokollow show a big distance while Hariri, IS9830, Wad Ahmed and Tetron show a small distance (Fig. 2A). 


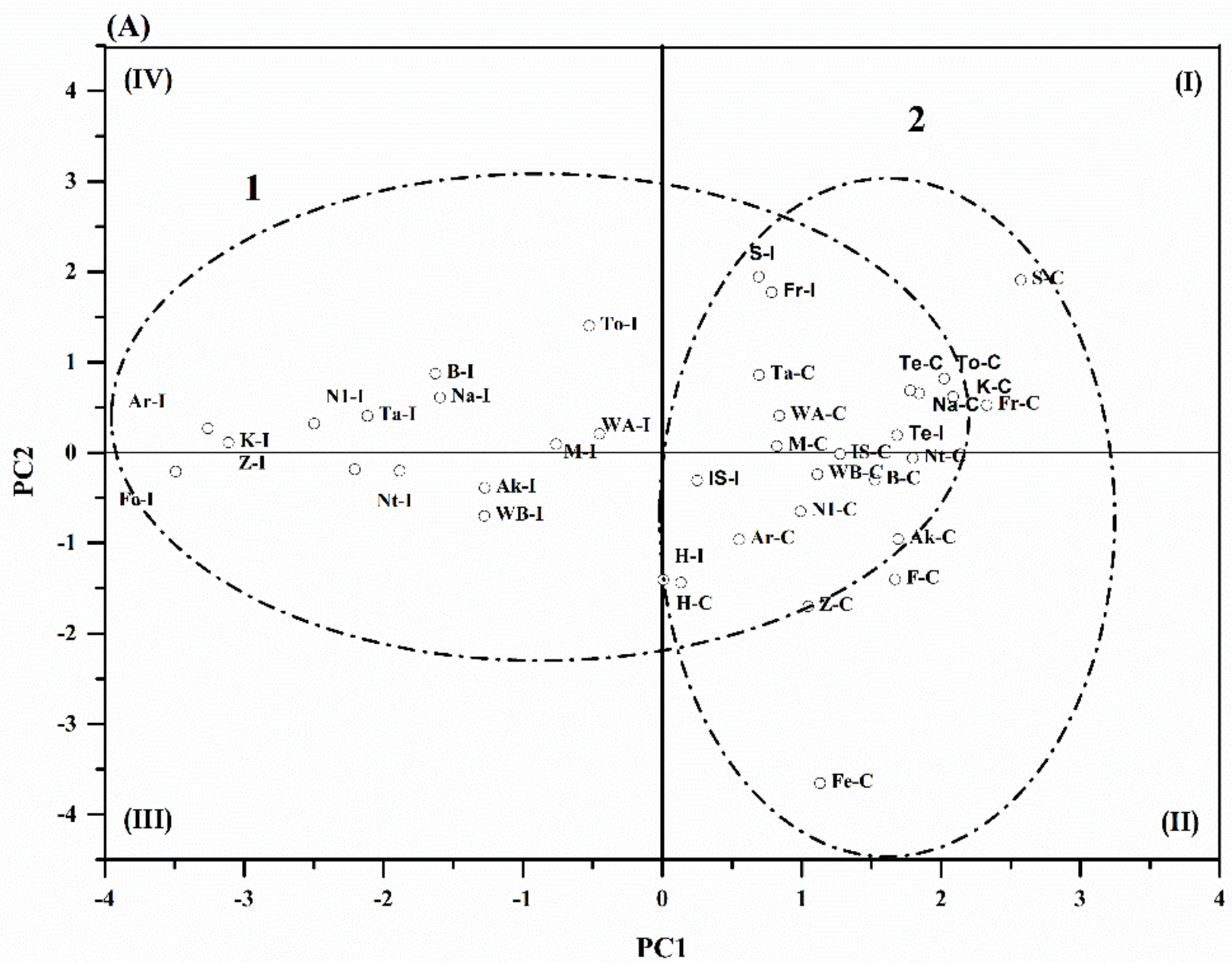


(B)

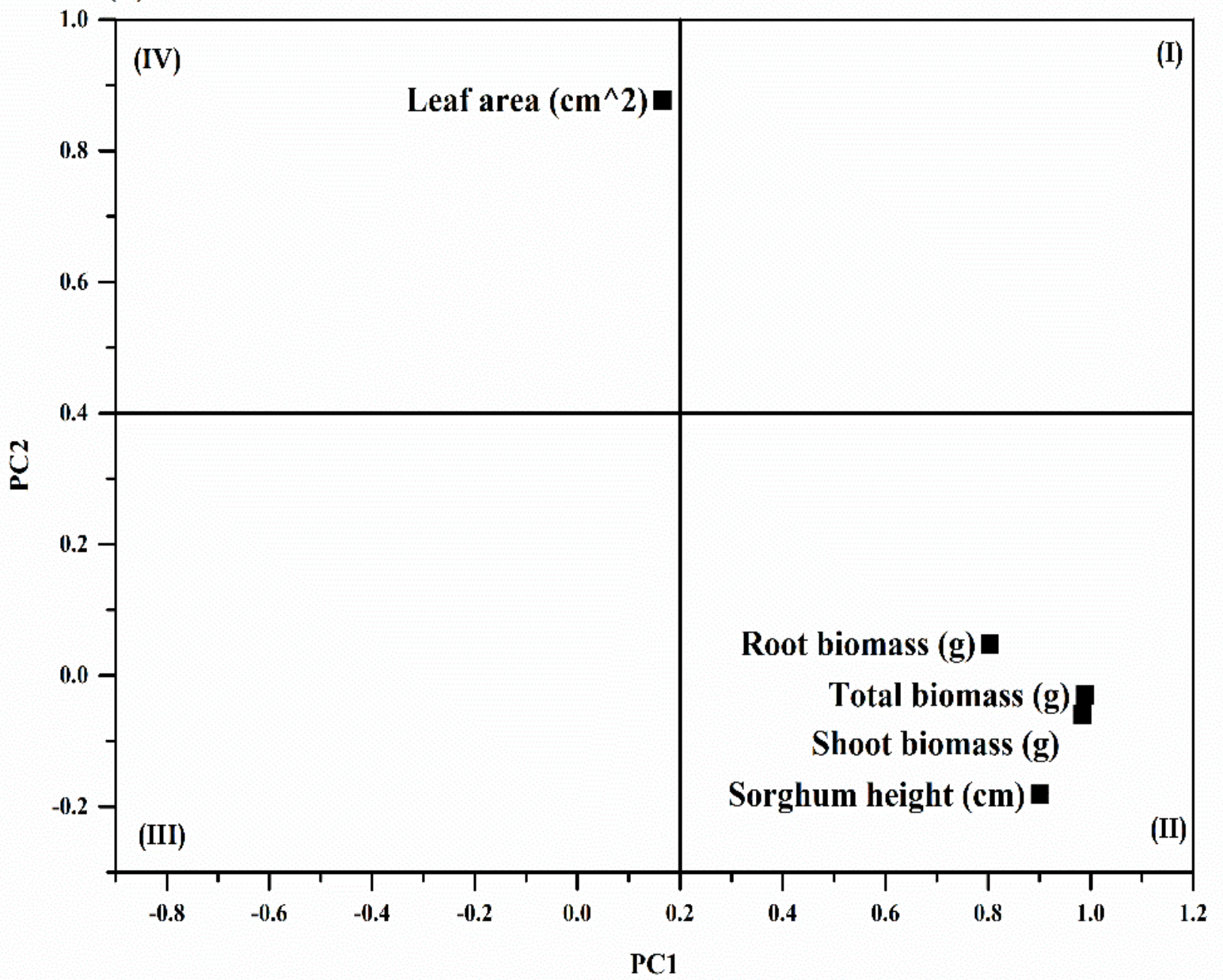

Fig. 2 PC1 versus PC2 (A) scores and (B) loadings plots based on morphological traits, showing differentiation between Striga-infected and Striga free control. Group legend: (1) Striga-infected, (2) Striga free control. In plot (A) genotype names are abbreviated, full names as follow Ak=Aklamoi, Ar=Arfa Gadamak, B= Botana, F= Fakimustahi, Fe= Feterita Geshaish, Fr= Framida, H= Hariri, IS= IS-9830, K= Korokollow, $\mathrm{M}=$ Mogud, $\mathrm{Na}=$ Najad, $\mathrm{Nt}=$ Naten, $\mathrm{N} 1=\mathrm{N} 13, \mathrm{~S}=\mathrm{SRN39}, \mathrm{Ta}=$ Tabat, $\mathrm{Te}=$ Tetron, $\mathrm{To}=$ Tokarawe, WA= Wad Ahmed, WB= Wad Baco, Z= Zahrat Elgadambalia. 
Table 3 Principal Component (PCS) analysis of morphological traits (A), photosynthesis traits and concentration of abscisic acid (B) showing Eigenvalues, variances and trait contribution to the total variation explained by the first two principal components.

\begin{tabular}{|c|c|c|}
\hline (A) & PC1 & PC2 \\
\hline Eigenvalue & 3.48 & 1.06 \\
\hline Individual & 0.69 & 0.21 \\
\hline \multirow[t]{2}{*}{ Cumulative (\%) } & 0.69 & 0.90 \\
\hline & \multicolumn{2}{|c|}{ trait loadings } \\
\hline Descriptor & $\mathrm{PC} 1$ & PC2 \\
\hline stem height $\mathrm{cm}$ & 0.88 & -0.30 \\
\hline root biomass (g) & 0.84 & -0.20 \\
\hline shoot biomass (g) & 0.98 & -0.06 \\
\hline total biomass (g) & 0.99 & 0.00 \\
\hline leaf area $\mathrm{cm} 2$ & 0.16 & 0.96 \\
\hline (B) & PC1 & PC2 \\
\hline Eigenvalue & 7.69 & 1.32 \\
\hline Individual & 76.9 & 10.0 \\
\hline \multirow[t]{2}{*}{ Cumulative } & 76.9 & 86.9 \\
\hline & \multicolumn{2}{|c|}{ trait loadings } \\
\hline Descriptor & PC1 & $\mathrm{PC} 2$ \\
\hline $\mathrm{A} \max \left(\mu \mathrm{mol} \mathrm{CO} \mathrm{CO}^{-2} \mathrm{~s}^{-1}\right)$ & 0.99 & -0.05 \\
\hline$g s\left(\mathrm{~mol} \mathrm{H}_{2} \mathrm{O} \mathrm{m}^{-2} \mathrm{~s}^{-1}\right)$ & 0.99 & 0.08 \\
\hline Evpt & 0.98 & -0.01 \\
\hline$\Phi C O 2\left(\mu \mathrm{mol} \mathrm{CO} 2 \mathrm{~mol}^{-1}\right)$ & 0.98 & -0.07 \\
\hline $\mathrm{Ci}\left(\mu \mathrm{mol} \mathrm{CO} \mathrm{mol}^{-1}\right)$ & -0.47 & 0.75 \\
\hline ETR & 0.98 & -0.05 \\
\hline $\mathrm{qP}$ & 0.93 & -0.04 \\
\hline ABA (pg/mg) & -0.66 & -0.55 \\
\hline
\end{tabular}

Amax: maximum rates of photosynthesis; gs: stomatal conductance; $\Phi \mathrm{CO} 2$ : carboxylation efficiency; Evpt: transpiration rate; $\mathrm{C} i$ : concentrations of $\mathrm{CO} 2$ in sub-stomatal cavity; ETR: electron transport rate; qP: photochemical quenching; ABA: abscisic acid. 
Striga infection also impacted physiological traits such as photosynthesis (Amax), stomatal conductance $(g s), \mathrm{CO}_{2}$ use efficiency $(\Phi \mathrm{CO} 2)$, the $\mathrm{CO}_{2}$ concentration in the sub-stomata cavity $(\mathrm{C} i)$, electron transport rate (ETR), Photosystem II efficiency (ФPS2) and photochemical quenching (qP) (Table S3). In addition, abscisic acid (ABA) concentration $(\mathrm{pg} / \mathrm{ml})$ in the leaf tissues of Striga-infected treatments displayed a significant $(P<0.05)$ increase compared to the respective Striga-free controls, except in Aklamoi, where a slight drop (13\%) in ABA concentration was observed (Table S3). Among the genotypes tested Mogud, Tetron and Naten exhibited the highest increase in ABA concentration (144-170\%) whereas Wad Ahmed, Najad, Hariri and SRN39 showed only 88, 67, 52 and 30\% higher ABA concentration than their respective controls, respectively (Table S3). Significant correlations were observed within and between morphological and physiological parameters and Striga number and biomass (Tables S6-S7). For example, total biomass positively correlated with plant height, shoot and root biomass, Amax, gs, ФCO2, Ci, ETR, ФPS2 and qP (Tables S4-S5).

To visualise the effects of Striga on the physiological values PCA was done (Fig. 3). PC1 and PC2, have Eigenvalues $>1$, explain a total $86 \%$ of the variation and provide the best visualization of the separation between the genotypes. PC1 accounts for $76 \%$ of the variation (Table 3B). Amax, gs, ФCO2, ETR, ФPS2 and qP had high positive loadings on $\mathrm{PC} 1$, while $\mathrm{C} i$ and $\mathrm{ABA}$ concentration presented the highest negative loadings in this $\mathrm{PC}$ (Table 3B; Fig. 3). PC2 accounts for $10 \%$ of the total variation (Table 3B). Descriptor $\mathrm{C} i$ has the highest positive loadings on this $\mathrm{PC}$, while Amax, stomatal conductance ( $g s), \mathrm{CO}_{2}$ use efficiency (ФCO2), Electron transport rate (ETR), Photosystem II efficiency ФPS2 and photochemical quenching (qP) presented null or negative loadings on this $\mathrm{PC}$ (Table 3B). ABA has a high negative loading on both PCs (Table 3B). Thus genotypes located in quadrant IV of the plot are generally characterized by relatively high values of $\mathrm{C} i$ and in III by relatively higher ABA concentrations. Genotypes clustered in the right of the plot have higher Amax, gs, ФCO2, Ci, ETR, ФPS2 and qP (Fig. 3A, B). Indeed, under control conditions, the genotypes are clustered in quadrants I and II, so have high Amax, gs, ФCO2, ETR,ФPS2 and qP and lower $\mathrm{C} i$ and ABA but upon Striga infection they all move to 
quadrants III and IV, having lower values of Amax, gs, ФCO2, ETR,ФPS2 and qP and higher $\mathrm{C} i$ and ABA (Fig 3). However, the magnitude of this shift from right to left is genotype-dependent. For some genotypes, like Naten and Aklamoi the shift is relatively large while for others such as Hariri, SRN39 and Tetron the shift is small. When comparing within genotypes with respect to the concentration of $\mathrm{CO}_{2}$ in the sub-stomatal cavity $(\mathrm{C} i)$ irrespective of Striga infection level genotypes falling in quadrat (I) exhibited significant $(P<0.05)$ build-up of $\mathrm{CO}_{2}(\mathrm{C} i)$ in the sub-stomatal cavity (Fig 3A; Table S3). Conversely genotypes falling in quadrat (II) and (III) exhibited deficiency in build-up of $\mathrm{CO}_{2}(\mathrm{C} i)$ in the sub-stomatal cavity (Fig 3B; Table S3). Similarly, Striga infection induced significant reductions in all photosynthesis related parameters except in values of $(\mathrm{C} i)$ which showed a decrease in some genotypes and an increase in others (Table S3; Fig. 5). 

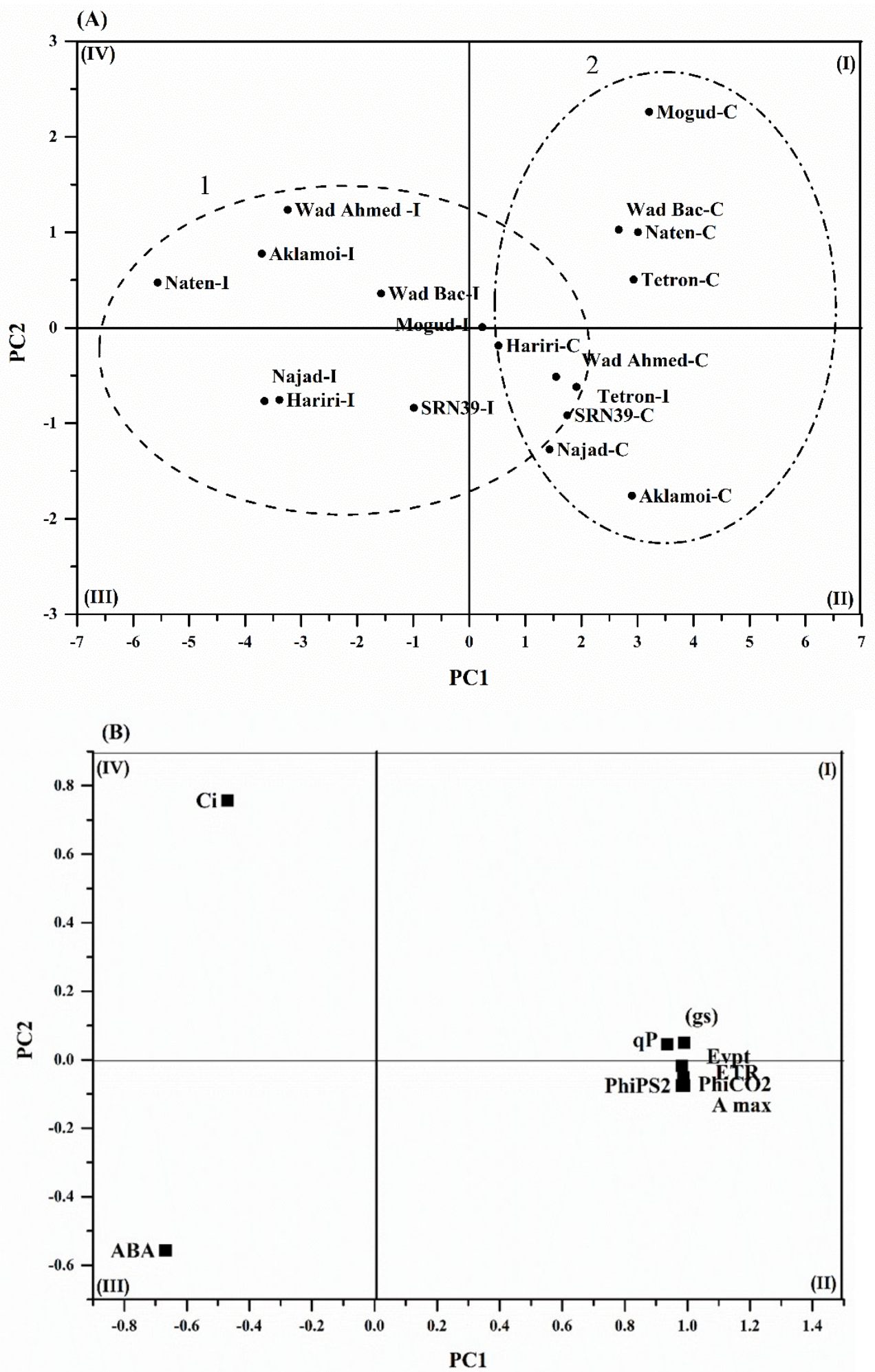

Fig. 3 PC1 versus PC2 (A) scores and (B) loadings plots based on physiological traits, showing differentiation between Striga-infected and Striga free control. Group legend: (1) Striga-infected, (2) Striga free control. 


\section{Relationship between Striga and morphological and physiological traits}

To further estimate the impact of Striga on sorghum, we evaluated the change in sorghum biomass and physiological parameters in relation to the biomass of Striga on that genotype. Leaf area showed high variability both within and between genotypes. Arfa Gadamak, Botana, Fakimustahi, Feterita Geshaish, Framida and Zahrat Elgadambalia displayed high (30-79\%) reductions, Aklamoi and N13 exhibited low (9-20\%) reductions in comparison to the respective controls. In contrast Hariri, IS9830, Korokollow, Mogud, Najad, Naten, SRN39, Tabat, Tetron, Tokarwe, Wad Ahmed and Wad Baco showed an increase by 14$65 \%$ in comparison to the respective Striga free controls (Table S2). LA was negatively correlated with emergent Striga number $(r=-0.26)$ and Striga biomass $(r=-0.22)(P<0.01)$ (Table S6). Irrespective of the level of Striga infection the extent of sorghum biomass reduction differed significantly between genotypes (Table S2). The relationship between Striga biomass and sorghum biomass reduction (of infected versus control plants) shows that there is a large variation between the genotypes in the extent of Striga infection as well as the impact of this infection on the host (Fig. 4). Genotypes above the regression line are more sensitive while genotypes falling below the line are more tolerant (Fig. 4; Table S2). Comparison of relative biomass losses between genotypes with similar infection levels

shows there are large differences between genotypes in the extent of the Striga effect. Despite high infection levels in genotypes e.g. Hariri, Wad Ahmed, Tetron, Mogud and to a lesser extent Framida, Tabat, N13, IS9830 and Wad Baco were less affected by Striga in terms of total biomass reduction than for example Fakimustahi, Korokollow, Feterita Geshaish, Arfa Gadamak, Najad and Zahrat Elgadambalia (Fig. 4). 


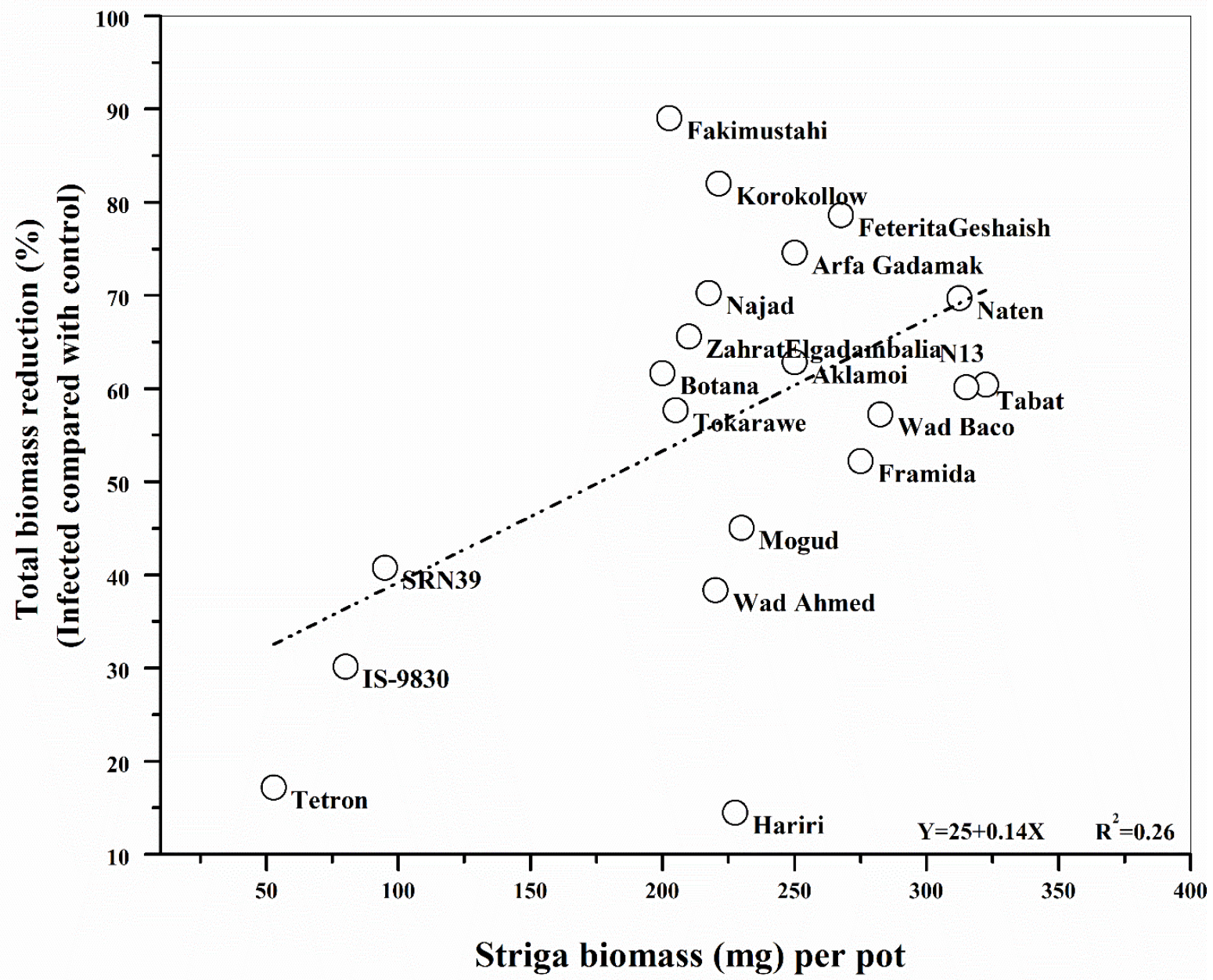

Fig. 4 Genetic variation in Striga-inflicted losses (\%) in total sorghum biomass relative to the Striga-free control plants at harvest, plotted against the total biomass of emerged Striga plants.

Fig. 5 shows the fitted linear relation between Amax, gs, ФCO2 and $\mathrm{C} i$ and Striga biomass. The regression line shows that the reduction in all parameters as a consequence of Striga infection increases with increasing Striga infection. However, the deviation from the regression line by the individual genotypes indicates that changes in these parameters varied significantly $(\mathrm{P}<0.05)$ among genotypes. Naten, Aklamoi, and Najad constantly incurred relatively the highest Striga-induced reductions in Amax, gs and ФCO2, while Wad Ahmed and Wad Baco incurred lower Striga-induced reductions. The resistant genotypes Tetron, SRN39 and Mogud constantly incurred relatively the least reduction in 
all photosynthetic parameters. The most notable genotype was Mogud as it showed, despite high Striga infection levels, the smallest reduction in Amax, gs and ФCO2. Similarly, Striga infection invariably decreased values of parameters derived from chlorophyll fluorescence including ETR, qP and DPS2 in all genotypes relative to the Striga free controls (Table S3). Tetron and SRN39 constantly incurred the lowest reduction in ETR, qP and ФPS2, while Aklamoi, Najad, Wad Baco and Wad Ahmed incurred the highest; Mogud and Hariri suffered moderate reductions.

\section{Tolerance Index}

To quantify the tolerance that is suggested by Figs 4 and 5, a Striga tolerance index (TI) was calculated based on the values of the total sorghum biomass produced under Striga infection relative to that produced under Striga free conditions (Table S8). Significant $(P<0.001)$ differences were observed among the genotypes with respect to their TI (Table S8). The TI correlated negatively with Striga biomass ( $\mathrm{r}=-0.26, P<0.01$; Fig. $6 \mathrm{~A})$ and positively with time of Striga emergence $(\mathrm{r}=0.57, P<0.05)$ (Table S1; Fig. 6B). Three groups can be identified in the scatter plot that correlates TI with Striga biomass (Fig. 6A). The first group, located in the top right part of the plot, includes genotypes Hariri, Mogud, Wad Ahmed, Wad Baco, Framida, Tabat that exhibit relatively high Striga infection combined with relatively high TI values. Those genotypes were able to endure/mitigate 

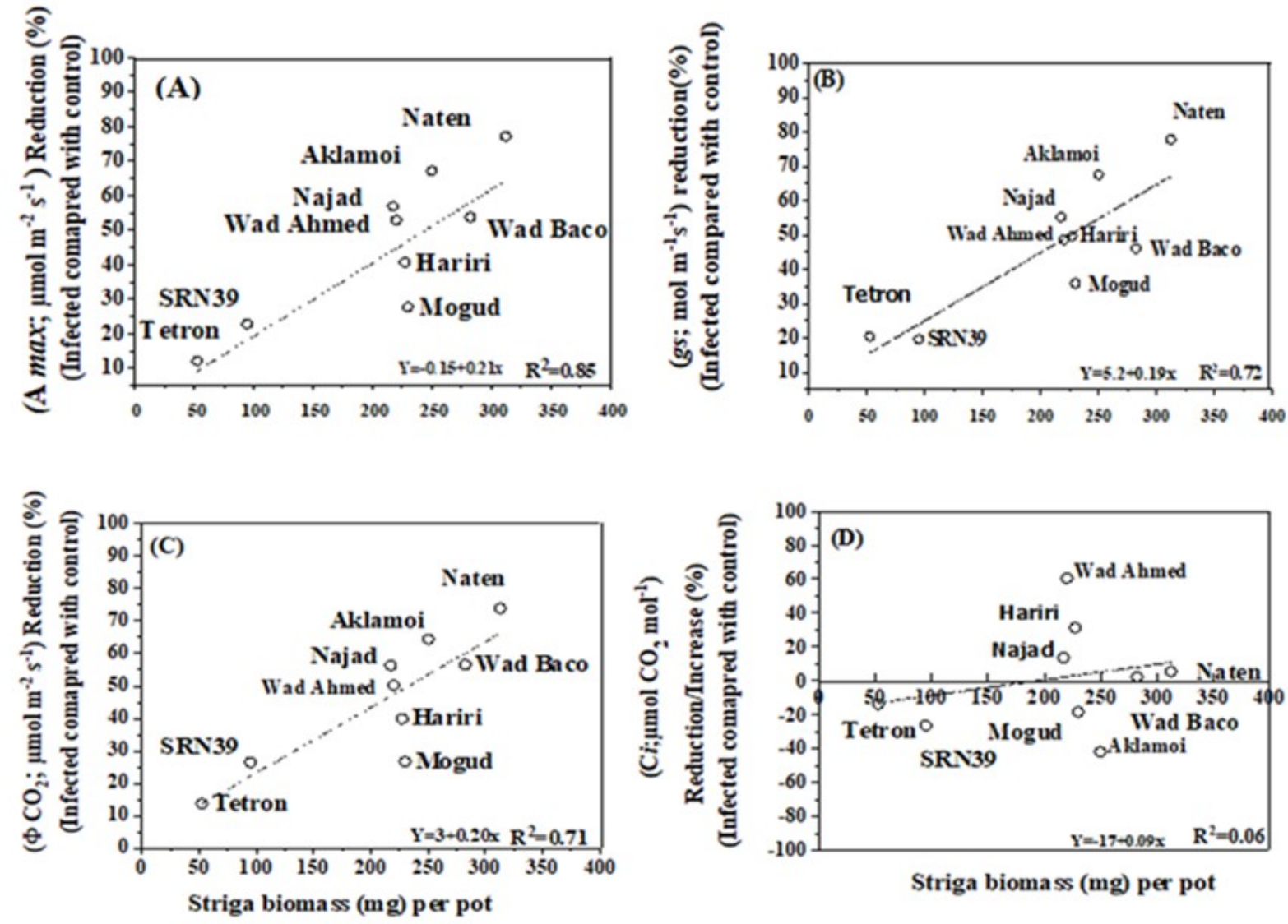

Fig. 5 Genetic variation in Striga-inflicted changes (\%) in physiological parameters (A) Change in maximum rate of photosynthesis; (B) Change in stomatal conductance; (C) Change in carboxylation efficiency; (D) Change in concentration of $\mathrm{CO}_{2}$ in the sub-stomatal cavity all relative to the Striga-free control plants, plotted against the total biomass of emerged Striga plants. 
Striga negative effects and thus incur small biomass losses hence can be characterised as tolerant. The second group, located at the bottom of the plot includes genotypes Fakimustahi, Feterita Geshaish, Korokollow, Arfa Gadamak, Aklamoi and Zhrat Elgadambalia that exhibit low TI values and are hence unable to endure/mitigate Striga negative effects and can be characterised as sensitive. The third group, located at the top left side of the plot, includes genotypes Tetron, SRN39 and IS-9830 that exhibited significantly lower Striga infection - apparent from the low Striga biomass, and can thus be denoted as resistant, while Tetron and SRN39 were also able to endure/mitigate Striga negative effects, making them also tolerant (Fig. 6A).

In Fig. 6B three groups can be identified. The first group, located in the top right part of the plot, includes genotypes Tetron, Hariri, Wad Ahmed, SRN39 and IS-9830 in which Striga emergence occurred more than 30 days after sowing and that have relatively high TI values. The second group, located in the middle of the plot includes genotypes Wad Baco, Mogud, Framida, Tokarawe, Botana, Najad, Naten, N13, Korkollow and Arfa Gadamak in which Striga emergence occurred about 25 days after sowing and of which some had a reasonably high TI e.g. Mogud, Framida and Tokarawe. The third group, located at the bottom left side of the plot, includes genotypes Tabat, Aklamoi, Feterita Geshaish, Fakimustahi and Zahrat Elgadambalia in which Striga emergence ocurred early which coincided with a low TI, with the exception of Tabat that has a reasonably high TI. 
(A)
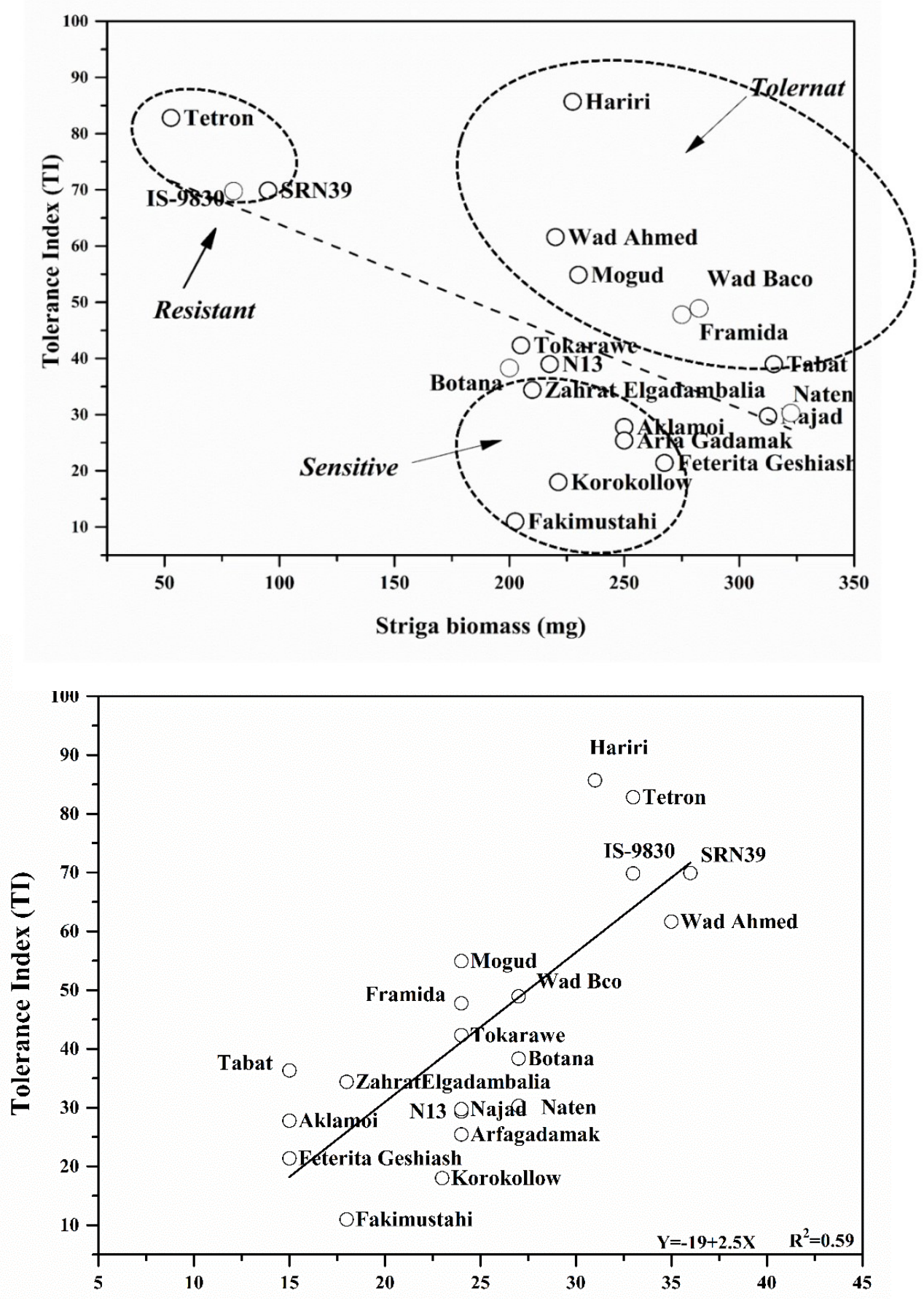

Days to to first Striga emergence

Fig. 6 A) Relationship between tolerance index (TI) and Striga biomass. B) The relationship between tolerance index (TI) and time to first Striga emergence. Data presented as means of 4 replicates $\pm \mathrm{SE}$. The line of best fit (Regression analysis) is indicated. 


\section{The relationship between strigolactones and tolerance index}

The peak areas of 5-deoxystrigol, orobanchol and sorgomol were used in PCA analysis to visualize the relationship between the strigolactones present in the root exudates of the different genotypes, their germination inducing activity, Striga infection per pot and their tolerance index (Fig. 7). The first two principal components had an Eigenvalue higher than one and explained $69 \%$ of the variation in the surveyed traits (Table S9). The first principle component, PC1, explained 53\% of the variation, with positive loadings for 5-deoxystrigol, germination stimulating activity, Striga plants per pot and a negative loading for orobanchol and tolerance index (Table S9). The second principle component (PC2) explained $15 \%$ of the total variation with positive loadings for orobanchol, sorgomol and germination stimulating activity and a negative loading for tolerance index, 5-deoxystrigol and Striga plants per pot (Table S9). In Fig. 7 according to the directions and angles of the vectors, 5-deoxystrigol exhibited a positive correlation with the germination stimulating activity and Striga plants per pot while orobanchol and tolerance index showed an inverse correlation with germination stimulating activity and Striga plants per pot (Fig. 7). Sorgomol showed a weak positive correlation with germination stimulating activity and Striga plants per pot and a negative correlation with orobanchol and particularly the tolerance index (Fig. 7). In addition to multivariate PCA, correlation analysis was used to investigate the relationship between the exudate concentrations of 5-deoxystrigol, sorgomol and orobanchol and the Striga tolerance index. The amounts of orobanchol in the root exudates significantly correlated positively with genotype tolerance index $(r=0.33 ; \mathrm{P}<$ 0.002) and negatively with Striga number and biomass, while amounts of 5-deoxystrigol and sorgomol showed a significant negative correlation with the tolerance index $(r=-0.31$ and $r=-0.32 ; \mathrm{P}<0.004$ and $\mathrm{P}<0.003$, respectively) (Table $\mathrm{S} 10$ ). Correlation analysis across all genotypes between strigolactone peak areas in the root exudates and numbers of emerged Striga plants and Striga biomass (mg) per pot, showed that 5-deoxystrigol significantly and positively correlated with emerged Striga numbers and Striga biomass $(\mathrm{r}=0.36 ; \mathrm{r}=0.38$; both $\mathrm{P}<0.01)$ (Table $\mathrm{S} 10$ ), while orobanchol, displayed a significant negative correlation with both Striga traits $(\mathrm{r}=-0.41$ and $\mathrm{r}=-0.43 ; \mathrm{P} \leq 0.05$ and $\mathrm{P} \leq 0.01$, 
respectively) (Table S10). Sorgomol showed a significant positive correlation but only with the numbers of emerged Striga plants $(\mathrm{r}=0.23 ; \mathrm{P}<0.05)$ (Table $\mathrm{S} 10)$.

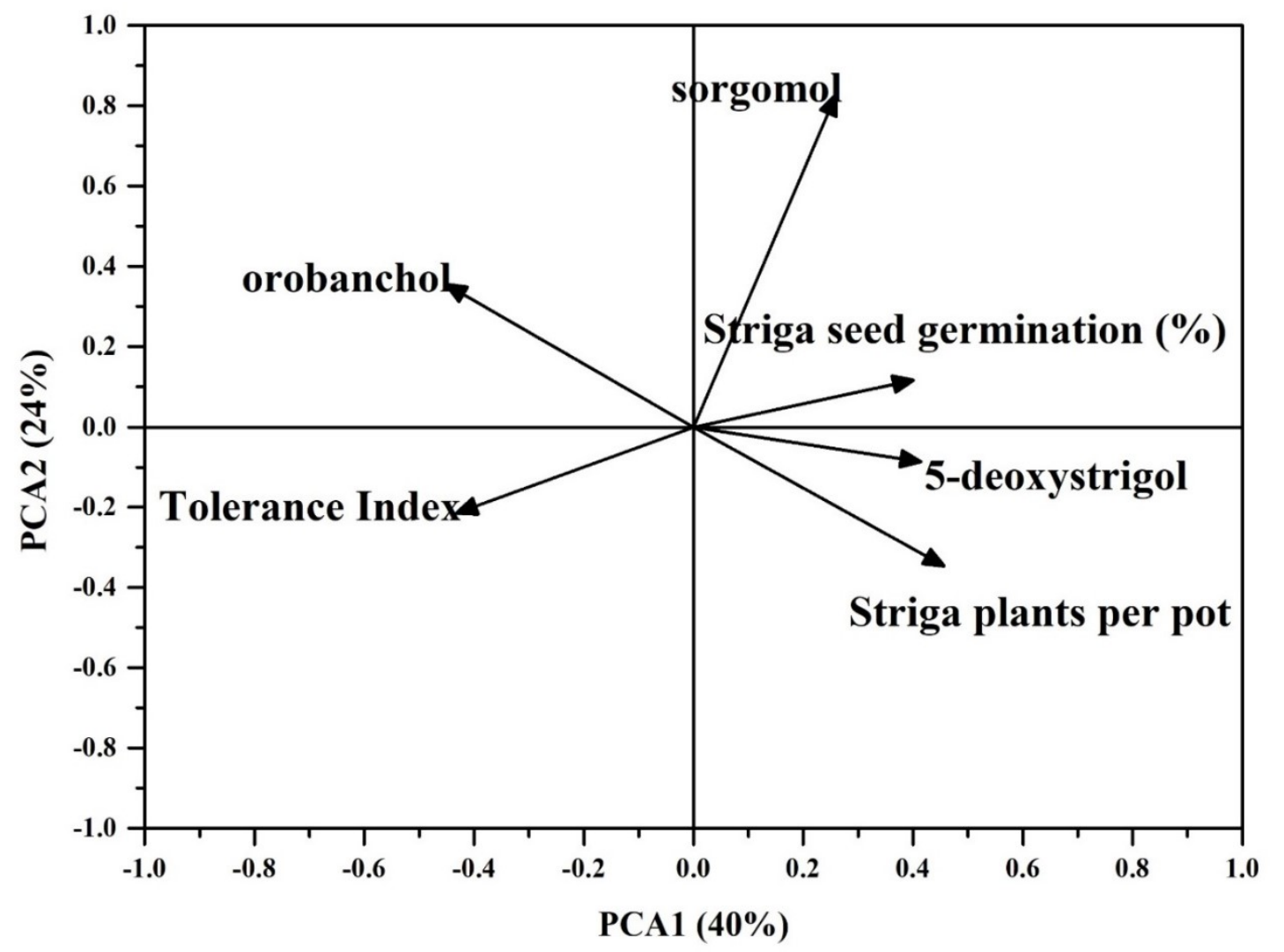

Fig. 7: Bi-plot of the first two components of a PCA based on strigolactone peak area showing relationship between strigolactone composition, genotype tolerance index, germination of Striga seeds as induced by root exudates and the number of emerged Striga plants per pot.

\section{Discussion}

Tolerance to Striga is defined as the ability of a crop genotype to endure or mitigate the negative impact of infection, which is reduction in morphological traits e.g. stem height and biomass and/or reduction in physiological traits e.g. photosynthetic capacity (Van Ast et al., 2002; Rodenburg and Bastiaans, 2008). In the present study we identified several genotypes that despite high Striga infection rates display lower than average reduction in crop morphological and physiological traits compared with the uninfected control as well as genotypes with higher than average reduction in these traits. Intriguingly, the lower impact of Striga on some genotypes (for example less affected photosynthetic capacity) 
coincided with delayed emergence of Striga on these genotypes and with exudation of the strigolactone orobanchol but not 5-deoxystrigol and sorgomol and vice versa. For example, the genotypes Mogud, Hariri, Wad Ahmed, Wad Baco, Framida, and Tokarawe supported late Striga emergence, and responded with much less severe reduction in sorghum height, shoot and total biomass despite quite high Striga numbers (Table S11; Fig. 4), whereas the genotypes Fakimustahi, Feterta Geshaish, Najad, Tabat, Naten, Aklamoi and N13 supported early (and high) Striga emergence and responded with a severe reduction in stem height, leaf area, and shoot and total biomass (Table S2). The differential magnitude of the damage displayed by the two groups of genotypes is congruent with the definition of tolerance to the parasite for the first group and sensitivity for the second group as defined by several authors (Doggett, 1984; Parker and Riches, 1993; Rodenburg and Bastiaans, 2011). Delayed Striga emergence and its association with reduced negative impact of the parasite on its host corroborate a previous report by Gurney et al., (1999) and is in accord with the findings of van Ast et al., (2006) who reported that delayed Striga emergence accounts for tolerance and reduction of deleterious effects of the parasite. Later attachment also is expected to diminish changes in host plant hormones which probably play an important role in altering growth and physiology following infection (Drenan and ElHewarith, 1972). Reduction and delay in Striga emergence, however, could also be attributed to resistance conferred by reduced germination and/or haustorium initiation and attachment (Mohamed et al., 2010; Mohemed et al 2016). Here we also observed the presence of a significant positive association between amounts of the strigolactone compound orobanchol exuded in the root exudates in relatively higher amounts in some genotypes and their tolerance index values. This was supported by the presence of a significant negative association between amounts of the same compound and emerged Striga numbers and biomass (Table S9). These results provide indirect evidence that orobanchol is increasing host tolerance and at the same time decreasing parasitism efficiency. A recent study in tomato also showed that a mutation in the strigolactone biosynthetic gene $C C D 8$ which results in the almost complete block of the production of orobanchol, the most abundant tomato strigolactone, results in increased sensitivity of 
tomato to infection by the broomrape, Phelipanche ramosa (Cheng et al., 2017). Cheng et al (2017) speculated that strigolactones may play a role in the inhibition of the infection process. In Arabidopsis exogenous application of the synthetic SL GR24 rescued the drought and salt stress sensitive phenotype of SL deficient mutants (max3-11 and max4-7), suggesting that SLs is a positive regulator of abiotic stress tolerance responses (Bu et al., 2014) and as we show here possibly also to biotic stresses such as Striga, which actually also induces drought stress symptoms in its hosts.

However, orobanchol is also one of the most active stimulants of AM fungi hyphal branching induction (Akiyama et al., 2010). These beneficial mycorrhizal fungi enhance plant uptake of inorganic phosphate $(\mathrm{Pi})$ and other mineral nutrients from the soil, stimulate plant growth, increase tolerance to drought and protect plant roots against pathogens and possibly against Striga (Smith and Gianinazzi-pearson, 1988; Ruizlozano et al., 1995; Alan, 2000; Veresoglou and Rillig, 2011). Lendzemo et al., (2007) showed that inoculating sorghum with AM fungi in pots or in the field resulted in a decrease in Striga infection and in significant reduction in performance of Striga in terms of Striga number, biomass and delayed its time of emergence. This effect was more clear in a tolerant cultivar than in a sensitive one indicating interaction with the genotype of the host. Here we showed that tolerant genotype such Wad Ahmed, Framida, Wad Baco produce more orobanchol than sensitive cultivars e.g Fakimustahi, Arfa Gadamak and Feterita Geshaish and that might have resulted in a better interaction with AM fungi which may have improved host tolerance and reduced parasitism efficiency. Therefore, from the positive association between tolerance index and time of Striga emergence and orobanchol content (Fig. 7B) it can be concluded that delayed emergence and/or late infection is/are, at least in part, responsible for (or a consequence of) the observed level of tolerance in some genotypes and that this possibly was influenced by exudation of high orobanchol/low 5-deoxystrigol levels.

The results clearly show that tolerance/sensitivity to Striga is not purely a consequence of a high Striga density (Figs. 4, 7). For instance, when comparing the genotypes Korokollow and Hariri, both support a similar biomass of Striga (about $225 \mathrm{mg} /$ pot). However, 100 
Korokollow responded with a $>80 \%$ reduction in sorghum biomass compared with the uninfected control, while Hariri responded with only 15\% biomass reduction (Fig. 4) thus suggesting significant tolerance to Striga infection for Hariri and a sensitive response for Korokollow (Fig. 7). The inverse correlations between Striga infection traits (Striga number, Striga biomass) and sorghum morphological traits (sorghum height, shoot biomass and total dry biomass (Table S6), does not support the report by Press et al., (1987) who claimed that the degree of infection is of less importance than the infection itself in determining the deleterious effects of Striga on host growth and physiology. The tolerance described here for Wad Ahmed was reported earlier by Babiker (2008) and for Mogud was formerly reported by Wilson-Jones (1953) (Fig. 7A). However, the presence of tolerance in Wad Baco and Hariri have not been reported so far although they are ranked by farmers as tolerant genotypes. Framida, Tetron, SRN39 and IS9830 are often described as resistant (Kambal, 1979; Ejeta et al., 2007) and this is confirmed in the present study (low Striga infection; Figs 4, 7). Our study helps to shed light on the conflicting reports on the assignment of these genotypes as resistant or tolerant (Elhiweris, 1987; Rodenburg et al., 2005). Fig. 7A clearly shows that SRN39 and IS-9830 are located on the regression line suggesting they have average Striga sensitivity; Tetron is clearly located above the line and could thus be called tolerant. In contrast to Tetron, Fakimustahi, Korokollow, Feterita Geshaish, Arfa Gadamak, Aklamoi and Zahrat Elgadambalia showed a strong reduction in biomass upon Striga infection and therefore had a low TI, identifying them as sensitive genotypes (Table S2; Fig.7A).

The PCA analysis revealed a number of morphological and physiological traits that exhibited major relevance for $\mathrm{PC} 1$ and a considerable number of these parameters showed very similar behavior (Table 3A-B; Figs 2, 3). However, LA, ABA and $\mathrm{C} i$ had greater relevance for PC2 (Figs 2, 3). Strong correlations between biomass accumulation and photosynthetic capacity present evidence of the importance of these traits in genotypic evaluation for Striga tolerance (Table S5). ABA displayed a negative correlation with photosynthesis related parameters and total biomass (Table S7). Indeed, a higher concentration of ABA was observed in all Striga infected treatments (Table S3). Striga, 
renowned for its high transpiration rate, predisposes its host to water stress and, as a consequence, increased ABA levels. The negative impact of $\mathrm{ABA}$ on photosynthesis is consistent with the effect of ABA on stomatal closure which decreases gas exchange and hence rate of photosynthesis. Stunting of the stem and reduction in LA in some of the susceptible/sensitive genotypes is consistent with previous reports that Striga reduces LA possibly in response to perturbation of the hormonal balance particularly between ABA and gibberellins, nutrient depletion and/or pathological effects (Drennan and El-Hiweris, 1979; Press et al., 1996; Frost et al., 1997; Watling et al., 2001). It is worth mentioning that dwarfism in sorghum has been linked to loss-of-function in genes involved in GA biosynthesis (Ordonio et al., 2014). Further, the high reduction in biomass accumulation could be explained by the acute deficiency in photo-assimilates resulting from the observed drop in photosynthetic capacity and stomatal conductance in infected plants.

A decrease in sorghum photosynthetic capacity due to Striga infection has been documented earlier (Gurney et al., 1995). However, the present study shows that the magnitude of the decrease is genotype dependent. For example, genotype Hariri, identified as tolerant, based on its high TI, despite the high Striga infection, retained $50-73 \%$ of its Amax, $\Phi \mathrm{CO}_{2}, \mathrm{ETR}, \Phi \mathrm{PS} 2$ and $\mathrm{qP}$ and displayed only $14 \%$ biomass reduction, whereas the Striga sensitive Naten with low TI, retained only 23-41\% of its leaf Amax, ФCO $_{2}, \mathrm{ETR}$, ФPS2 and qP and exhibited 69\% reduction in biomass compared to the respective control (Tables S2-3). The genotype Tetron was rather exceptional as it showed the lowest reductions (12-21\%) in all assessed parameters including total biomass. This is partially due to tolerance (Fig. 7) but also to resistance. Also SRN39 which is resistant but does not display tolerance maintained (71-84\%) of its Amax, ФCO2, ETR, ФPS2 and qP and exhibited only $40 \%$ reduction in biomass, whereas Aklamoi, which maintained only 33$55 \%$ of its Amax, ETR, ФCO2 and ФPS2 showed $62 \%$ reduction in biomass (Tables S23). The results are consistent with several reports (Gurney et al., 1995; Van Ast et al., 2000; Rodenburg et al., 2008) showing that Striga-infected tolerant and tolerant/resistant sorghum genotypes display relatively less reduced photosynthetic capacity compared to their sensitive congeners. 
The strong correlations $(r=0.38-0.52)$ (Table S5) between total biomass and Amax, $g s$, ФCO2, ETR, ФPS2 and qP suggest that the slight reductions in the morphological traits observed in the tolerant genotypes were largely due to maintainability of relatively higher photosynthetic capacity (Table S3). On the other hand, the considerable to severe reductions in the morphological traits observed with some of the sensitive genotypes (Table S2) are largely due to reduction in photosynthetic capacity rather than merely a result of nutrient and/or water deficiencies.

The present study showed increased ABA concentrations in the shoots of Striga infected genotypes compared to the respective controls (Table S3). Increased levels of ABA due to Striga infection and its impact on stomatal conductance and leaf expansion have been documented (Frost et al., 1997a). However, it could be argued that stomatal limitations in addition to impairment of carboxylation are the main reasons for reduced rate of photosynthesis because for all genotypes the rate of photosynthesis and stomatal conductance are strongly correlated (Table S5). The significant buildup of $\mathrm{CO}_{2}$ in substomatal cavities (Table S3) and its inverse association with $\Phi C O 2$ (Fig. S1) suggest that, independent of stomatal limitations, Striga infection negatively impacts photosynthetic metabolism.This notion differs from the proposition made by Watling and Press, (2001) that in sorghum Striga infection initiates a decline in rate of photosynthesis, but not in ФCO2. Further, Frost et al., (1997b) reported no impact of infection on activity of photosynthetic enzymes and suggested that lower values of stomatal conductance are the principal cause of lowering the rate of photosynthesis in Striga infected sorghum. Further, they suggested that Striga attachment to the roots might induce localized water stress or a wounding response and that the $\mathrm{ABA}$ originating in plant roots, independent of leaf water potential, causes stomatal closure. However,Watling et al., (2001) indicated that in C3 plants e.g. rice, $\Phi \mathrm{CO} 2$ is a function of photosynthetic enzyme ribulose-1, 5-bisphosphate carboxylase/oxygenase (Rubisco) activity while in C4 plants e.g. sorghum it depends on the activity of phosphoenolpyruvate carboxylase (PEPC) activity. For C3 plants Amax is determined by RuBP regeneration capacity. While in $\mathrm{C} 4$ plants Amax is determined by PEPC regeneration capacity and/or Rubisco activity (von Caemmerer and Farquhar, 1981; 
von Caemmerer and Furbank, 1999). In rice it has been shown that Striga infection decreases both $\Phi \mathrm{CO} 2$ and Amax and Rubisco content was lower in infected rice plants (Watling and Press, 2000). In this study in sorghum based on analysis of $\mathrm{C} i, \Phi C O 2$ and Amax we demonstrated that Striga infection decreases both $\Phi C O 2$ and Amax. However, a change in phosphoenolpyruvate carboxylase (PEPC) content will still have to be established to reinforce our findings. Previously in sorghum several authors reported a decline in Amax due to Striga infection, however, no decrease in $\Phi \mathrm{CO} 2$ in sorghum due to Striga infection has so far been documented (Ramlan and Graves, 1996). The present study demonstrated that there is clear genotypic variation in the stomatal response to Striga infection in sorghum (Fig 5B). The results suggest that in the genotypes Wad Ahmed, and Hariri, in which $\Phi \mathrm{CO} 2$ was less affected by $\mathrm{C} i$, the stomatal response may be less sensitive to changes in ABA concentration than in the genotypes Aklamoi and Naten, in which carboxylation efficiency relative to Wad Ahmed, and Hariri was reduced by increased $\mathrm{CO}_{2}$ concentration in sub-stomatal cavities (Fig 5D). However, measurements on activities of photosynthetic enzymes in response to in-situ ABA resulting from Striga-infection and/or exogenously applied ABA may help in ascertaining or refuting the role of the hormone in tolerance and/or sensitivity of sorghum to the parasite.

The present study suggests that decreased photosynthesis carboxylation efficiency, reflected in higher $\mathrm{CO}_{2}$ concentration in sub-stomatal cavities, and reduced stomatal conductance, possibly due to increased $\mathrm{ABA}$, are responsible, at least in part, for the significant reduction in photosynthetic capacity and consequently for the strong reduction in biomass accumulation, particularly in sensitive genotypes. Finally, the study identified Hariri, Tetron, Mogud, Wad Ahmed, Framida, Tabat and Wad Baco as genotypes displaying a significant level of tolerance to the root parasitic weed S. hermonthica. On these genotypes Striga displayed delayed emergence, while a number of these genotypes that we tested for photosynthesis also maintained relatively high photosynthetic capacity despite a still quite high Striga infection. These genotypes may facilitate identification of genes conferring tolerance to the parasite and may further serve as donors in breeding programs. The present study further suggested strigolactones may serve as an indicator for 
and/or play a role in tolerance and corroborates earlier reports on the validity of photosynthetic measurements as tools for in situ screening for host plant tolerance to Striga. In addition, the study proposes variation in carboxylation efficiency [ФCO2] as a further tool for discrimination between Striga tolerant and sensitive genotypes. Doing these measurements under conditions of controlled Striga infection would improve the sensitivity of this method to detect differences in tolerance.

\section{Acknowledgements}

The authors acknowledge funding by the Nuffic Fellowship Program, the Netherlands and the Wageningen sandwich fellowship (to NEM), the Netherlands Organization for Scientific Research (to HJB, VICI grant 865.06.002 and equipment grant 834.08.001), the European Research Council for ERC Advanced grant CHEMCOMRHIZO (670211 to HJB) and the Bill and Melinda Gates Foundation (Promise to HJB). We would like to thank Peter van der Putten from crop system analysis, Wageningen University for providing help with the photosynthesis measurements and data recording. The authors would like to thank Dr. Altahir Ibrahim Mohamed, (Plant Genetic Resource Unit), Agricultural Research Corporation, Wad Medani, Sudan for providing seeds of sorghum genotypes. 


\section{References}

Akiyama, K., Matsuzaki, K., and Hayashi, H. (2005). Plant sesquiterpenes induce hyphal branching in arbuscular mycorrhizal fungi. Nature 435, 824-827.

Akiyama, K., Ogasawara, S., Ito, S., and Hayashi, H. (2010). Structural requirements of strigolactones for hyphal branching in AM Fungi. Plant Cell Physiol. 51, 1104-1117.

Alan, G. (2000). Arbuscular mycorrhizal fungi, Collembola and plant growth. Trends in Ecology and Evolution. $15,369-372$.

Adetimirin, V., Aken Ova, M., and Kim, S. (2000). Effects of Striga hermonthica on yield components in maize. The Journal of Agricultural Science 135(2), 185-191.

Babiker, A. (2008). Striga control in Sudan: An integrated approach. In: Leslie, J.F. (edit) Sorghum and Millets Diseases, Iowa State Press. Pp 159-163.

Bebawi, F.F. (1981). Intraspecific physiological variants of Striga hermonthica. Experimental Agriculture, 17(04), 419-423.

Bouwmeester, H.J., Matusova, R., Zhongkui, S. \& Beale, M.H (2003). Secondary metabolite signaling in hostparasitic plant interactions. Curr. Opin. Plant Biol. 6, 358-364

Bu, Q.Y., Lv, T.X., Shen, H., Luong, P., Wang, J., Wang, Z.Y., Huang, Z.G., Xiao, L.T., Engineer, C., Kim, T.H., et al. (2014). Regulation of drought tolerance by the F-box protein MAX2 in Arabidopsis. Plant Physiol. 164, 424-439.

Clark, L.J., Shawe, K.G., Hoffmann, G., and Stewart, G.R. (1994). The effect of Striga hermonthica (Del.) Benth. infection on gas-exchange characteristics and yield of a sorghum host, measured in the field in Mali. Journal of Experimental Botany 45(2), 281-283.

Dangi, O., and IRA, B. (1989). Screening of advanced sorghum lines for resistance to Striga hermonthica in Cameroon. Striga, Improved Management in Africa: Proceedings of the FAO/OAU All-Africa Government Consultation on Striga Control, Maroua, Cameroon, 20-24 October 1988 (96), 170.

Doggett,H.,(1984) Striga:its biology and control: an overview. Striga biology and control/edited by ES Ayensu...[et al.] ICSU Press, Paris, FR.

Drennan, D., and El Hiweris, S. (1979). Changes in growth regulating substances in Sorghum vulgare infected by Striga hermonthica, in: Proceedings of the second symposium of parasitic weeds. North Carolina State University, 144-155.

Efron, Y. (1993). Screening maize for tolerance to Striga hermonthica. Plant Breeding 110(3), 192-200.

Ejeta, G. (2007).The Striga scourge in Africa: a growing pandemic. Integrating new technologies for Striga control: towards ending the witch-hunt, World Scientific, Singapore, 3-16.

Ejeta, G., Rich, P.J., and Mohamed, A. (2007). Dissecting a complex trait to simpler components for effective breeding of sorghum with a high level of Striga resistance. Integrating new technologies for Striga control-towards ending the witch hunt. World Scientific, Singapore, 87-98.

Ejeta, G., A. Mohammed, P. Rich, A. Melake Berhan, T. L. Housley and D. E. Hess, 2000. Selection for specific mechanisms for resistance to Striga in sorghum. In: B. I. G. Haussmann, D. E. Hess, M. L. Koyama, L. Grivet, H. F. W. Rattunde and H. H. Geiger, Breeding for Striga resistance in cereals: proceedings of a workshop held at IITA, Ibadan, Nigeria, pp. 29-40

Frost, D., Gurney, A., Press, M., and Scholes, J. (1997a). Striga hermonthica reduces photosynthesis in sorghum: the importance of stomatal limitations and a potential role for ABA? Plant, Cell \& Environment 20(4), 483-492.

Gebremedhin, W., Goudriaan, J., and Naber, H. (2000). Morphological, phenological and water-use dynamics of sorghum varieties (Sorghum bicolor) under Striga hermonthica infestation. Crop Protection 19(1), 61-68. 
Graves, J., Press, M., and Stewart, G. (1989). A carbon balance model of the sorghum-Striga hermonthica hostparasite association. Plant, Cell \& Environment 12(1), 101-107.

Grenier, C., Ibrahim, Y., Haussmann, B.I., Kiambi, D., and Ejeta, G. (2007). Marker-assisted selection for Striga resistance in sorghum. Integrating New Technologies for Striga Control, Towards Ending the Witchhunt. Singapore: World Scientific Publishing, 159-172.

Gurney, A., Press, M., and Scholes, J. (1999). Infection time and density influence the response of sorghum to the parasitic angiosperm Striga hermonthica. New Phytologist 143(3), 573-580.

Gurney, A., Press, M., and Scholes, J. (2002). Can wild relatives of sorghum provide new sources of resistance or tolerance against Striga species? Weed Research 42(4), 317-324.

Gurney, A.L., Press, M.C., and Ransom, J.K. (1995). The parasitic angiosperm Striga hermonthica can reduce photosynthesis of its sorghum and maize hosts in the field. Journal of Experimental Botany 46(12), 1817-1823.

Haussmann, B. I. G., D. E. Hess, H. G. Welz and H. Geiger, 2000. Improved methodologies for breeding Strigaresistant sorghums. Field Crops Research 66, 195211.

Hiweris, S. (1987). Nature of resistance to Striga hermonthica (Del.) Benth. parasitism in some Sorghum vulgare (Pers.) cultivars. Weed research 27(5), 305-311.

Kambal, A.a.M., B.M. (1979). Breeding sorghum cultivars for resistance to Striga. Tech. Prog. Report. No.1. IDRC. A.E. Kambal (Edit.), PP.6-25.

Kim, S. (1991). Breeding for tolerance and general resistance in maize: a novel approach to combating Striga in Africa. Nairobi, Kenya, 168-176.

Kling, J. G., J. M. Fajemisin, B. Badu Apraku, A. Diallo, A. Menkir and A. Melake Berhan, 2000. Striga resistance breeding. In: B. I. G. Haussmann, D. E. Hess, M. L. Koyama, L. Grivet, H. F. W. Rattunde and H. H. Geiger (eds). Breeding for Striga resistance in cereals: Proceedings of a workshop held at IITA, Ibadan, Nigeria, pp. 103-118.

Lendzemo, V.W., Kuyper, T.W., Matusova, R., Bouwmeester, H.J., and Van Ast, A. (2007). Colonization by arbuscular mycorrhizal fungi of sorghum leads to reduced germination and subsequent attachment and emergence of Striga hermonthica. Plant Signal Behav. 2, 58-62.

Manual, L. I. C. O. R. (2005). Using the LI-6400 portable photosynthesis system.

Mboob, S. (1989). A regional programme for Striga control in West and Central Africa. Proceedings of the FAO/OAU All African Government Consultation on Striga Control, Marova. FAO, Rome, 190-194.

Mohamed, A.H., Housley, T., and Ejeta, G. (2010). An in vitro technique for studying specific Striga resistance mechanisms in sorghum. African Journal of Agricultural Research 5(14), 1868-1875.

Mohemed, N., Charnikhova, T., Bakker, E. J., Ast, A., Babiker, A. G., \& Bouwmeester, H. J. (2016). Evaluation of field resistance to Striga hermonthica (Del.) Benth. in Sorghum bicolor (L.) Moench. The relationship with strigolactones. Pest Management Science, 72 (11), 2082-2090.

Lopez-Raez, J. A., Kohlen, W., Charnikhova, T., Mulder, P., Undas, A. K., Sergeant, M. J., \& Bouwmeester, H. (2010). Does abscisic acid affect strigolactone biosynthesis?. New Phytologist, 187(2), 343-354.

Ordonio, R.L., Ito, Y., Hatakeyama, A., Ohmae-Shinohara, K., Kasuga, S., Tokunaga, T., et al. (2014). Gibberellin deficiency pleiotropically induces culm bending in sorghum: an insight into sorghum semidwarf breeding. Scientific Reports 4, 5287.

Oswald, A., and Ransom, J. (2004). Response of maize varieties to Striga infestation. Crop Protection 23(2), 8994.

Parker, C. (2009). Observations on the current status of Orobanche and Striga problems worldwide. Pest management science 65(5), 453-459.

Parker, C., and Riches, C.R. (1993). Parasitic weeds of the world: biology and control. CAB international.

Press, M., and Graves, J. (1995). Parasitic plants. Springer Science \& Business Media. 
Press, M.C., Gurney, A.L., Frost, D.L., Scholes, J.D., 1996. How does the parasitic angiosperm Striga hermonthica influence host growth and carbon relations? In: Advances in Parasitic Plant Research. Eds M.T. Moreno, J.I. Cubero, D. Berner, D. Joel, L.J. Musselman, C. Parker, Proceedings of the Sixth International Parasitic Weed Symposium, Cordoba, Spain, pp. 303-310

Press, M.C., Scholes, J.D., and Watling, J.R. (1999). Parasitic plants: physiological and ecological interactions with their hosts. Physiological plant ecology, 175-197.

Press, M.C., Tuohy, J.M., and Stewart, G.R. (1987). Gas exchange characteristics of the sorghum-Striga hostparasite association. Plant Physiology 84(3),

Ramaiah KV (1984) Patterns of Striga resistance in sorghum and millets with special emphasis on Africa. In: Proceedings 1984 International Workshop on the Biology and Control of Striga, Dakar (eds E.S. Ayensu et al.), 71-92. ICSU Press, Paris, France.814-819.

Ramlan, M. F., \& Graves, J. D. (1996). Estimation of the sensitivity to photoinhibition in Striga hermonthicainfected sorghum. Journal of experimental botany, 47(1), 71-78.

Rodenburg, J. (2005). The role of sorghum genotype in the interaction with the parasitic weed Striga hermonthica. Wageningen University and Research Centre.

Rodenburg, J., and Bastiaans, L. (2011). Host-plant defence against Striga spp.: reconsidering the role of tolerance. Weed Research 51(5), 438-441.

Rodenburg, J., Bastiaans, L., and Kropff, M.J. (2006). Characterization of host tolerance to Striga hermonthica. Euphytica 147(3), 353-365.

Rodenburg, J., Bastiaans, L., Schapendonk, A.H., van der Putten, P.E., van Ast, A., Dingemanse, N.J., et al. (2008). CO2-assimilation and chlorophyll fluorescence as indirect selection criteria for host tolerance against Striga. Euphytica 160(1), 75-87.

Rodenburg, J., Bastiaans, L., Weltzien, E., and Hess, D. (2005). How can field selection for Striga resistance and tolerance in sorghum be improved? Field Crops Research 93(1), 34-50.

Ruizlozano, J.M., Azcon, R., and Gomez, M. (1995). Effects of arbuscular-mycorrhizal Glomus species on drought tolerance - physiological and nutritional plant-responses. Appl. Environ. Microb. 61, 456-460.

Smith, S.E., and Gianinazzi-pearson, V. (1988). Physiological Interactions between Symbionts in vesiculararbuscular mycorrhizal plants. Annu. Rev. Plant.Phys. 39, 221-244.

Spallek, T., Mutuku, M., and Shirasu, K. (2013). The genus Striga: a witch profile. Molecular plant pathology 14(9), 861-869.

Stewart, G.R., Press, M.C., Graves, J.D., Nour, J.J., and Wylde, A. (1991). A physiological characterization of the host parasite association between Sorghum bicolor and Striga hermonthica and its implication for Striga control. Combating Striga in Africa. Kim SK (Ed) pages, 45-54.

Watling, J. R., and M. C. Press. Impacts of infection by parasitic angiosperms on host photosynthesis. Plant Biology 3.03 (2001): 244-250.

Van Ast, A., 2006. The influence of time and severity of time and severity of Striga infection on the Sorghum bicolor-Striga hermonthica association. PhD Thesis, Wageningen University, Wageningen, The Netherlands.

Van Ast, A., Bastiaans, L., and Kropff, M.J. (2000). A comparative study on Striga hermonthica interaction with a sensitive and a tolerant sorghum cultivar. Weed Research 40(6), 479-493.

Veresoglou, S.D., and Rillig, M.C. (2011). Suppression of fungal and nematode plant pathogens through arbuscular mycorrhizal fungi. Biol. Lett. 8, 214-217. 


\section{Supplementary tables}

Table S1 Pearson Correlation between emerged number of Striga/pot, total Striga biomass/pot, days to first Striga emergence and tolerance Index (IT).

\begin{tabular}{lllll}
\hline & Striga Numbers & Striga biomass (mg) & DFE. & TI. \\
\hline Striga numbers & 1 & $0.43^{*}$ & $-0.53^{*}$ & $-0.135 \mathrm{NS}$ \\
Striga biomass (mg) & 1 & $-0.56^{*}$ & $-0.263^{*}$ \\
DFE (time in days) & & 1 & $0.57^{*}$ \\
\hline
\end{tabular}

**Correlation significant at $\mathrm{P}<0.01$ (2-tailed); * Significant at $\mathrm{P}<0.05$ (2-tailed); NS =nonsignificant.

Table S2 Change (\%) in morphological traits in sorghum upon Striga infection relative to a Striga-free control. Data presented are means of 4 replicates. Negative values indicates a decrease upon Striga infection; positive values indicates an increase. The significance of differences was determined by a one-way ANOVA. Means within a column followed by the same letter are not significantly different according to the Duncan's pairwise comparisons $(P<0.05)$.

\begin{tabular}{lllllll}
\hline No & Genotype & LA & Ph & shoot & root & total biomass \\
\hline 1 & Aklamoi & -9.19 & $-74.40 \mathrm{ab}$ & $-73.48 \mathrm{ab}$ & $-28.60 \mathrm{abcd}$ & $-62.86 \mathrm{abcdef}$ \\
2 & Arfa Gadamak & -31.1 & $-80.41 \mathrm{a}$ & $-86.73 \mathrm{a}$ & $-39.69 \mathrm{abcd}$ & $-74.58 \mathrm{abcd}$ \\
3 & Botana & -30.1 & $-81.24 \mathrm{a}$ & $-67.51 \mathrm{ab}$ & $-33.59 \mathrm{abcd}$ & $-61.65 \mathrm{abcdef}$ \\
4 & Fakimustahi & -47.1 & $-90.25 \mathrm{a}$ & $-93.02 \mathrm{a}$ & $-80.16 \mathrm{a}$ & $-89.02 \mathrm{a}$ \\
5 & Feterita Geshaish & -79.5 & $-88.34 \mathrm{a}$ & $-89.56 \mathrm{a}$ & $-54.53 \mathrm{abc}$ & $-78.61 \mathrm{abc}$ \\
6 & Framida & -24.3 & $-66.29 \mathrm{abcd}$ & $-74.33 \mathrm{ab}$ & $-3.08 \mathrm{~d}$ & $-52.23 \mathrm{bcdef}$ \\
7 & Hariri & +9.8 & $-17.70 \mathrm{a}$ & $-23.61 \mathrm{de}$ & $-4.68 \mathrm{~d}$ & $-14.49 \mathrm{~g}$ \\
8 & IS-9830 & +14.4 & $-20.00 \mathrm{a}$ & $-32.04 \mathrm{cde}$ & $-25.02 \mathrm{abcd}$ & $-30.16 \mathrm{fg}$ \\
9 & Korokollow & +38.0 & $-79.85 \mathrm{a}$ & $-86.11 \mathrm{a}$ & $-73.45 \mathrm{a}$ & $-82.00 \mathrm{ab}$ \\
10 & Mogud & +22.2 & $-36.41 \mathrm{de}$ & $-60.11 \mathrm{abc}$ & $-8.58 \mathrm{~d}$ & $-45.07 \mathrm{cdefg}$ \\
11 & Najad & +20.4 & $-70.87 \mathrm{ab}$ & $-81.84 \mathrm{ab}$ & $-34.09 \mathrm{abcd}$ & $-70.25 \mathrm{abcde}$ \\
12 & Naten & +20.0 & $-75.68 \mathrm{ab}$ & $-75.08 \mathrm{ab}$ & $-54.52 \mathrm{abc}$ & $-69.71 \mathrm{abcde}$ \\
13 & N13 & -20.4 & $-81.59 \mathrm{a}$ & $-80.24 \mathrm{ab}$ & $-36.67 \mathrm{abcd}$ & $-60.44 \mathrm{abcde}$ \\
14 & SRN39 & +39.2 & $-38.47 \mathrm{de}$ & $-33.87 \mathrm{cde}$ & $-10.92 \mathrm{~d}$ & $-40.75 \mathrm{defg}$ \\
15 & Tabat & +65.5 & $-74.93 \mathrm{ab}$ & $-76.92 \mathrm{ab}$ & $-17.86 \mathrm{bcd}$ & $-60.12 \mathrm{abcdef}$ \\
16 & Tetron & +36.0 & $-10.3 \mathrm{a}$ & $-17.22 \mathrm{e}$ & $-17.86 \mathrm{bcd}$ & $-17.18 \mathrm{~g}$ \\
17 & Tokarawe & +5.5 & $-79.89 \mathrm{a}$ & $-74.00 \mathrm{ab}$ & $-18.05 \mathrm{bcd}$ & $-57.67 \mathrm{abcdef}$ \\
18 & Wad Ahmed & +31.0 & $-48.15 \mathrm{bcd}$ & $-49.78 \mathrm{bcd}$ & $-8.23 \mathrm{~d}$ & $-38.36 \mathrm{efg}$ \\
19 & Wad Baco & +44.0 & $-42.02 \mathrm{cde}$ & $-70.25 \mathrm{ab}$ & $-25.15 \mathrm{abcd}$ & $-57.23 \mathrm{abcdef}$ \\
20 & ZahratElgadambalia & -49.1 & $-65.98 \mathrm{abcd}$ & $-74.31 \mathrm{ab}$ & $-42.09 \mathrm{abcd}$ & $-65.61 \mathrm{abcde}$ \\
\hline
\end{tabular}

*LA: leaf area $\left(\mathrm{cm}^{2}\right)$; Ph: Stem height $(\mathrm{cm})$; shoot, root and total biomass $(\mathrm{g})$ total plant biomass $(\mathrm{g})$. 
Table S3 Change (\%) in physiological traits in sorghum upon Striga infection relative to Striga-free control. Data presented as means of 4 replicates \pm SE. Negative values indicate a decrease upon Striga infection; positive values an increase. The significance of genotypic differences were determined by a one-way ANOVA. Means within a column followed by the same letter are not significantly different according to the Duncan's pairwise comparisons $(P<0.001)$.

\begin{tabular}{|c|c|c|c|c|c|c|c|c|c|}
\hline Genotype & $A \max$ & $(g s)$ & Evap & ФCO2 & $\mathbf{C i}$ & ETR & ФPS2 & $(q \mathrm{P})$ & $\begin{array}{l}\text { ABA } \\
(\mathrm{pg} / \mathrm{ml})\end{array}$ \\
\hline Aklamoi & $-67.16 a$ & $-67.54 a$ & $-66.99 a b$ & $-64.40 a$ & $-41.97 a$ & $-59.52 a$ & $-59.48 a$ & $-44.91 a$ & $-13.04 i$ \\
\hline Hariri & $-40.70 a b$ & $-49.72 a b$ & $-45.79 a b c$ & -39.89ab & $+31.29 \mathrm{~h}$ & $-34.20 a b$ & -33.92ab & $-27.24 a b$ & $+107.72 d$ \\
\hline Mogud & $-27.77 a b$ & $-35.90 a b$ & $-32.08 b c$ & $-26.63 a b$ & $-18.38 b$ & $-34.00 a b$ & -32.88ab & $-21.90 a b$ & $+147.70 \mathrm{~b}$ \\
\hline Najad & $-56.87 a b$ & $-55.17 a b$ & $-54.03 a b c$ & $-56.32 a b$ & $+13.56 \mathrm{f}$ & $-54.09 a b$ & $-54.06 a b$ & $-53.97 a$ & $+66.60 \mathrm{~g}$ \\
\hline Naten & $-77.10 \mathrm{a}$ & $-77.71 \mathrm{a}$ & $-76.82 a$ & $-73.93 a$ & $+5.55 \mathrm{e}$ & $-69.80 a$ & $-69.81 a$ & $-58.78 a$ & $+169.68 \mathrm{a}$ \\
\hline SRN39 & $-22.97 a b$ & $-19.58 b$ & $-16.44 c$ & $-26.44 a b$ & $+22.23 \mathrm{~g}$ & -28.95ab & $-28.96 a b$ & $-24.68 a b$ & $+31.20 \mathrm{~h}$ \\
\hline Tetron & $-12.18 b$ & $-20.53 b$ & $-18.22 c$ & $-13.69 b$ & $-2.28 c$ & $-1.90 b$ & $-2.05 b$ & $-4.36 \mathrm{~b}$ & $+144.05 c$ \\
\hline Wad Ahmed & $-52.87 a b$ & $-48.53 a b$ & $-48.50 a b c$ & $-50.13 a b$ & $+60.48 \mathrm{i}$ & $-53.37 a b$ & $-53.42 a b$ & $-47.49 a$ & $+88.15 \mathrm{e}$ \\
\hline Wad Baco & $-52.82 a b$ & $-46.04 a b$ & $-34.38 a b c$ & $-56.59 a b$ & $+2.48 \mathrm{~d}$ & $-43.01 a b$ & $-42.97 a b$ & $-36.57 a b$ & $+88.20 \mathrm{e}$ \\
\hline
\end{tabular}

Amax: Maximum rate of photosynthesis ( $\left.\mu \mathrm{mol} \mathrm{CO}_{2} \mathrm{~m}^{-2 \mathrm{~s}-1}\right)$; gs : stomatal conductance $\left(\mathrm{mol} \mathrm{H}_{2} \mathrm{O} \mathrm{m}^{-2} \mathrm{~s}^{-1}\right)$; $\Phi C O 2$ : carboxylation efficiency $\left(\mu \mathrm{mol} \mathrm{CO} \mathrm{Cm}^{-2 \mathrm{~s}-1}\right) ; \mathrm{Ci}\left(\mu \mathrm{mol} \mathrm{CO} \mathrm{Cm}^{-2 \mathrm{~s}-1}\right)$ : concentrations of $\mathrm{CO}_{2}$ in sub-stomatal cavity; ETR : Electron transport rate; ФPS2: Photosystem II efficiency; qP : photochemical quenching; ABA: ABA concentration in leaf tissue (pg/mg). 
Table S4 Phenotypic Pearson Correlation within and between sorghum morphological traits and tolerance index.

\begin{tabular}{llllll}
\hline & Total $(\mathrm{g})$ & Shoot $(\mathrm{g})$ & Root $(\mathrm{g})$ & $\mathrm{Ph}(\mathrm{cm})$ & leaf area $\left(\mathrm{cm}^{2}\right)$ \\
\hline Total $(\mathrm{g})$ & 1 & $0.945^{* *}$ & $0.764^{* *}$ & $0.670^{* *}$ & $0.291^{* *}$ \\
& & & & & \\
Shoot $(\mathrm{g})$ & 1 & $0.475^{* *}$ & $0.792^{* *}$ & $0.115 \mathrm{NS}$ \\
$\begin{array}{l}\text { Root }(\mathrm{g}) \\
\text { Ph }(\mathrm{cm})\end{array}$ & & 1 & $0.168 \mathrm{NS}$ & $0.544^{* *}$ \\
$\begin{array}{l}\text { leaf area }\left(\mathrm{cm}^{2}\right) \\
\text { tolerance index }\end{array}$ & $0.859^{* *}$ & $0.883^{* *}$ & $0.501^{* *}$ & $0.76^{* *}$ & $0.08 \mathrm{NS}$ \\
\end{tabular}

-LA: leaf area $\left(\mathrm{cm}^{2}\right)$; Ph (cm): Stem height in (cm); Shoot (g): shoot biomass (g); Root (g): root biomass $(\mathrm{g})$; Total $(\mathrm{g})$ : total plant biomass $(\mathrm{g})$; **Correlation is significant at 0.01 level (2tailed); * Significant at 0.05 level (2-tailed); NS :non-significant. 
Table S5 Pearson correlation within and between sorghum physiological traits and total biomass.

\begin{tabular}{|c|c|c|c|c|c|c|c|c|c|}
\hline & bi & $\mathrm{A} \max$ & $g s$ & $\Phi \mathrm{CO} 2$ & $\mathrm{C} i$ & $\mathrm{ABA}$ & ETR & ФPS2 & $\mathrm{qP}$ \\
\hline bi(g) & 1 & $0.512^{* *}$ & $0.52^{* *}$ & $0.51^{* *}$ & -0.10 & $-0.13 \mathrm{NS}$ & $0.525^{* *}$ & $0.509^{* *}$ & $0.38^{*}$ \\
\hline$A(\max )$ & & 1 & $0.94^{* *}$ & $0.98^{* *}$ & $-0.56^{* *}$ & $-0.09 \mathrm{NS}$ & $0.956^{* *}$ & $0.95^{* *}$ & $0.92^{* *}$ \\
\hline$(g s)$ & & & 1 & $0.92^{* *}$ & $-0.42^{*}$ & $-0.11 \mathrm{NS}$ & $0.940^{* *}$ & $0.93^{* *}$ & $0.90^{* *}$ \\
\hline ФCO2 & & & & 1 & $-0.54^{* *}$ & $-0.08 \mathrm{NS}$ & $0.94^{* *}$ & $0.95^{* *}$ & $0.92^{* *}$ \\
\hline $\mathrm{C} i$ & & & & & 1 & $-0.24 \mathrm{NS}$ & $-0.47^{* *}$ & $-0.47^{* *}$ & $-.430^{*}$ \\
\hline $\mathrm{ABA}$ & & & & & & 1 & 0.01 & $0.02 \mathrm{NS}$ & $-.029 \mathrm{NS}$ \\
\hline ETR & & & & & & & 1 & $0.94^{* *}$ & $0.94^{* *}$ \\
\hline ФPS2 & & & & & & & & 1 & $0.94^{* *}$ \\
\hline $\mathrm{qP}$ & & & & & & & & & 1 \\
\hline
\end{tabular}

Table S6. Pearson correlation between Striga infection traits; mean emerged Striga number/pot, total Striga biomass and sorghum morphological traits.

\begin{tabular}{lll}
\hline & Striga number/pot & Striga biomass/pot $(\mathrm{mg})$ \\
\hline Stem height & $-0.070 \mathrm{NS}$ & $-0.453^{* *}$ \\
Leaf area $\left(\mathrm{cm}^{2}\right)$ & $-0.268^{*}$ & $-0.223^{*}$ \\
Shoot biomass $(\mathrm{g})$ & $-0.290^{* *}$ & $-0.478^{* *}$ \\
Root biomass $(\mathrm{g})$ & $-0.287^{* *}$ & $-0.033 \mathrm{NS}$ \\
Total biomass & $-0.330^{* *}$ & $-0.307^{* *}$ \\
\hline
\end{tabular}

${ }^{* *}$ Correlation is significant at $\mathrm{P}<0.01$ (2-tailed); ${ }^{*}$ Significant at $\mathrm{P}<0.05$ (2-tailed); NS =nonsignificant 
Table S7. Pearson correlation between Striga infection traits; mean emerged Striga/pot, total Striga biomass and sorghum morphological traits.

\begin{tabular}{lll}
\hline & Striga number/pot & Striga biomass/pot (mg) \\
\hline Amax & $-0.31 \mathrm{NS}$ & $-0.30 \mathrm{NS}$ \\
$(g s)$ & $-0.32 \mathrm{NS}$ & $-0.29 \mathrm{NS}$ \\
$\Phi \mathrm{CO} 2$ & $-0.33 \mathrm{NS}$ & $-0.32 \mathrm{NS}$ \\
$\mathrm{C} i$ & $0.14 \mathrm{NS}$ & $-0.09 \mathrm{NS}$ \\
$\mathrm{ETR}$ & $-0.33 \mathrm{NS}$ & $-0.33 \mathrm{NS}$ \\
$\Phi P S 2$ & $-0.33 \mathrm{NS}$ & $-0.33 \mathrm{NS}$ \\
qP & $-0.26 \mathrm{NS}$ & $-0.19 \mathrm{NS}$ \\
ABA & $0.26 \mathrm{NS}$ & $-0.23 \mathrm{NS}$ \\
\hline
\end{tabular}

NS: the correlation is non-significant. Amax: Maximum rate of photosynthesis $\left(\mu \mathrm{mol} \mathrm{CO} \mathrm{Cm}^{-2}\right.$ $\mathrm{s}^{-1}$ ); gs : stomatal conductance ( $\mathrm{mol} \mathrm{H}_{2} \mathrm{O} \mathrm{m}^{-2} \mathrm{~s}^{-1}$ ); $\Phi \mathrm{CO} 2$ : carboxylation efficiency $\left(\mu \mathrm{mol} \mathrm{CO}_{2}\right.$ $\left.\mathrm{m}^{-2 \mathrm{~s}-1}\right) ; \mathrm{C} i\left(\mu \mathrm{mol} \mathrm{CO} \mathrm{Cm}^{-2 \mathrm{~s}-1}\right)$ : concentrations of $\mathrm{CO}_{2}$ in sub-stomatal cavity; ETR : Electron transport rate; ФPS2: Photosystem II efficiency; qP : photochemical quenching; ABA: ABA concentration in leaf tissue $(\mathrm{pg} / \mathrm{mg})$ 
Table S8. Genetic variation in Striga tolerance index (TI). The significance of genotypic differences was determined by a one-way ANOVA. Means within a column followed by the same letter are not significantly different according to the Duncan's pairwise comparison $(\mathrm{P}<0.001)$.

\begin{tabular}{lll}
\hline No & genotype & (TI) \\
\hline 1 & Aklamoi & $37.8 \mathrm{bcde}$ \\
2 & Arfa Gadamak & $25.4 \mathrm{cde}$ \\
3 & Botana & $38.3 \mathrm{cde}$ \\
4 & Fakimustahi & $10.9 \mathrm{f}$ \\
5 & Feterita Geshaish & $21.4 \mathrm{de}$ \\
6 & Framida & $47.8 \mathrm{abcd}$ \\
7 & Hariri & $85.7 \mathrm{a}$ \\
8 & IS-9830 & $69.8 \mathrm{ab}$ \\
9 & Korokollow & $18.0 \mathrm{ef}$ \\
10 & Mogud & $54.9 \mathrm{abc}$ \\
11 & Najad & $29.7 \mathrm{cde}$ \\
12 & Naten & $30.2 \mathrm{cde}$ \\
13 & N13 & $39.3 \mathrm{bcde}$ \\
14 & SRN39 & $59.9 \mathrm{abc}$ \\
15 & Tabat & $39.88 \mathrm{abcd}$ \\
16 & Tetron & $82.82 \mathrm{a}$ \\
17 & Tokarawe & $42.3 \mathrm{abcd}$ \\
18 & Wad Ahmed & $61.6 \mathrm{abc}$ \\
19 & Wad Baco & $48.9 \mathrm{abcd}$ \\
20 & Zahrat Elgadambalia & $34.4 \mathrm{cde}$ \\
\hline
\end{tabular}

(TI) Striga tolerance index: 100x(Sorghum total biomass under Striga infection/ Sorghum total biomass under Striga-free condition)

Table S9. Pearson Correlation between strigolactone peak area, tolerance Index (TI) and emerged Striga number and biomass (mg) per pot.

\begin{tabular}{llll}
\hline & 5-deoxystrigol & sorgomol & orobanchol \\
\hline Tolerance Index & $-0.31^{* *}$ & $-0.32^{* *}$ & $0.33^{* *}$ \\
5-deoxystrigol & 1 & 0.18 & -0.19 \\
sorgomol & 0.18 & 1 & -0.14 \\
orobanchol & -0.19 & -0.14 & 1 \\
Striga biomass (mg) per pot & $0.38^{* *}$ & 0.11 & $-0.43^{* *}$ \\
Striga emerged plants per pot & $0.36^{* *}$ & $0.23^{*}$ & $-0.41^{* *}$ \\
\hline
\end{tabular}

** Correlation is significant at $\mathbf{P}<0.05$ (1-tailed). 
Table S10. Strigolactone production in the 20 sorghum genotypes. Levels of 5-deoxystrigol, sorgomol and orobanchol in root exudates of sorghum genotypes. C18 and silica purified root exudates were analysed using liquid chromatography coupled with tandem mass spectrometry (LC-MS/MS). Peak area means of 4 replicates. Least significant differences of means at $\mathrm{P}=0.05$ were determined by ANOVA. Means within a column followed by the same letter(s) are not significantly different according to the Duncans' pairwise comparison $(\mathrm{P}<0.05)$.

\begin{tabular}{|c|c|c|c|c|}
\hline No & Genotype & 5-deoxystrigol* & sorgomol* & orobanchol* \\
\hline 1 & Aklamoi & 113.83 abcdefghi & 2.65 defghji & 0.28 efghji \\
\hline 2 & Arfa Gadamak & 95.94 abcdefgh & $0.00 \mathrm{~m}$ & $0.08 \mathrm{jk}$ \\
\hline 3 & Botana & 32.60 abcdefhgi & $0.09 \mathrm{klm}$ & $1.92 \mathrm{cdef}$ \\
\hline 4 & Fakimustahi & 260.84 abcdef & $269.87 \mathrm{ab}$ & $0.07 \mathrm{jk}$ \\
\hline 5 & Feterita Geshaish & $262.19 \mathrm{abcd}$ & 0.80 efhgjki & 0.57 defghi \\
\hline 6 & Framida & 189.15 abcdefhgi & $0.75 \mathrm{jikl}$ & $7.56 \mathrm{bc}$ \\
\hline 7 & Hariri & 2.03 kijl & $9.45 \mathrm{~cd}$ & 0.05 cdefe \\
\hline 8 & IS-9830 & 0.121 & $0.00 \mathrm{~m}$ & $28.65 \mathrm{~cd}$ \\
\hline 9 & Korokollow & 14.59 cdehfgji & $0.00 \mathrm{~m}$ & 0.23 defghi \\
\hline 10 & Mogud & 8.49 efhigj & $0.01 \mathrm{~lm}$ & 0.14 defghi \\
\hline 11 & N13 & $817.20 \mathrm{a}$ & $3.45 \mathrm{cdef}$ & 0.59 cdefghi \\
\hline 12 & Najad & 524.29 abcdef & $6.58 \mathrm{~cd}$ & $1.97 \mathrm{cdef}$ \\
\hline 13 & Naten & 10.08 defhgji & $19.82 \mathrm{bc}$ & 0.26 defghi \\
\hline 14 & SRN39 & 3.05 hjki & $5.71 \mathrm{cde}$ & $197.15 \mathrm{ab}$ \\
\hline 15 & Tabat & $557.55 \mathrm{abc}$ & $2.93 \mathrm{cdefg}$ & 0.58 cdefg \\
\hline 16 & Tetron & $2.616 \mathrm{jkl}$ & $0.00 \mathrm{~m}$ & $182.63 \mathrm{a}$ \\
\hline 17 & Tokarawe & 191.95 fhgji & 4.48 defhgi & 0.41 efghji \\
\hline 18 & Wad Ahmed & 244.43 abcdef & $1.73 \mathrm{~m}$ & $2.56 \mathrm{cdef}$ \\
\hline 19 & Wad Baco & 3.18 hgijk & 1.68 cdefg & $0.48 \mathrm{cdefg}$ \\
\hline 20 & Zahrat Elgadambalia & 86.20abcdefgh & $4.43 \mathrm{cde}$ & 0.35 cdefgh \\
\hline
\end{tabular}

*Peak area $\mathbf{x 1 0 ^ { 3 }}$ 


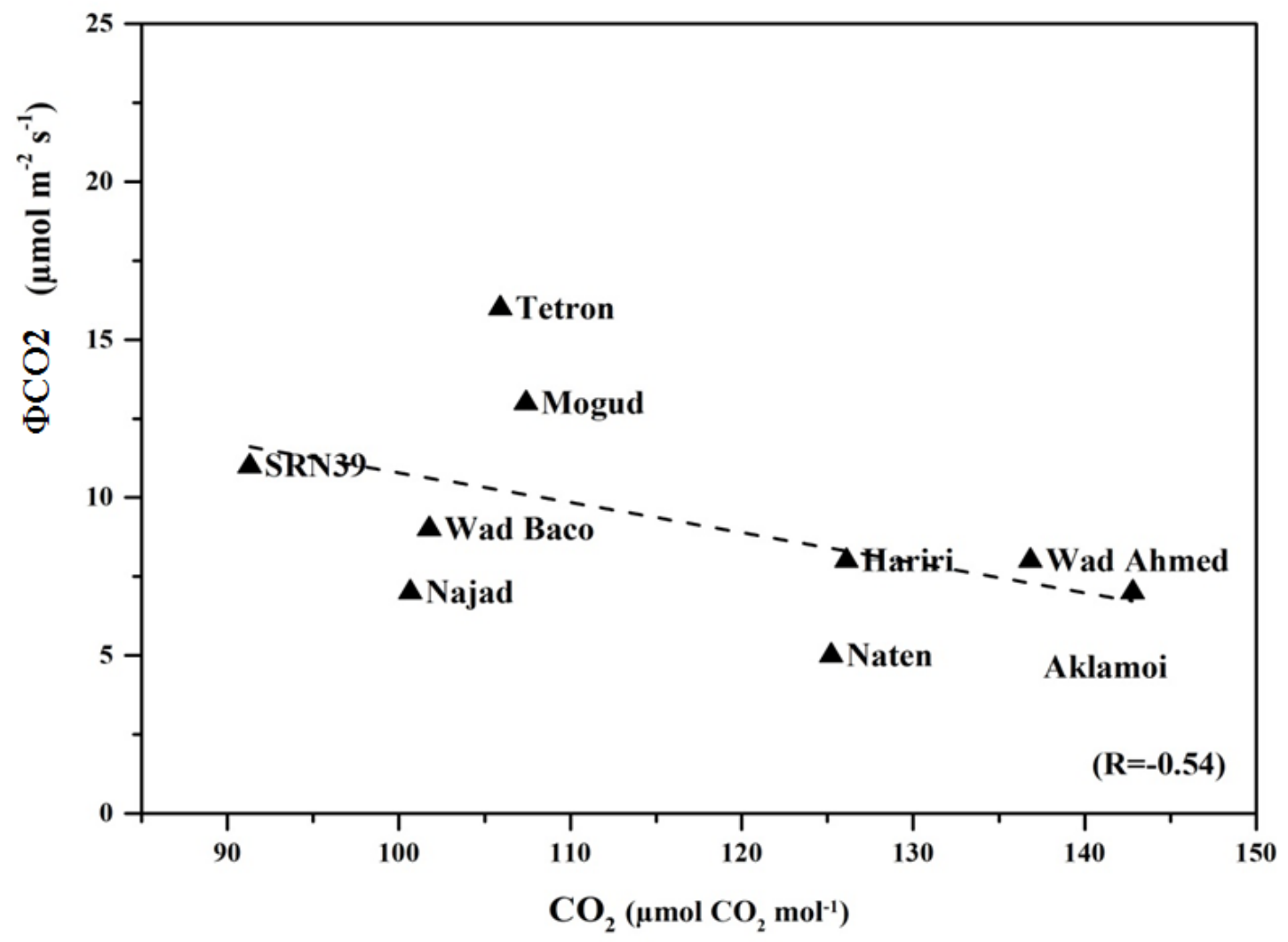

Fig. S1. Relationship between $\mathrm{CO}_{2}$ use efficiency and $\mathrm{CO}_{2}$ concentration in sub-stomatal cavity. Data are means of 3 replicates $\pm \mathrm{SE}$. The line of best fit (Regression analysis) is indicated. 


\title{
Chapter 4
}

\section{The role of strigolactones in Striga hermonthica control in sorghum by non-host intercrops}

Nasreldin M. Ahmed, Tatsiana Charnikhova, Abdelgabar G.T. Babiker and Harro J. Bouwmeester.

\begin{abstract}
Background: In the dryer parts of sub-Saharan Africa the parasitic weed Striga hermonthica (Del.) Benth. is one of the major constraints and threats to production of sorghum, maize and millet. The infection process by Striga starts with germination of its seed, which is induced by germination stimulants secreted by the host root. After germination a haustorium is formed with which Striga attaches to and penetrates into the host roots and develops a connection with the host vascular system. The use of non-host crops - that induce germination but cannot be infected by Striga - as intercrops or in crop rotation is a strategy to diminish the parasite seed bank and help reducing yield losses in Striga impacted crops. In this study, which is designed to explore the roles of strigolactones in this approach of Striga control in sorghum, we analysed the strigolactone content and germination stimulatory activity of the root exudates of sorghum, sesame, groundnut and pearl millet, as well as their Striga infection in pot and rhizotron experiments.

Results: The sesame and groundnut cultivars investigated produced the strigolactones, 5deoxystrigol, orobanchol and didehydro-orobanchol isomer like, while, in addition, the sesame cultivars also produced a solanacol-like strigolactone. Multivariate and correlation analyses between strigolactones and Striga germination and infection suggest that the Striga suicidal germination that occurred in the presence of an intercrop delayed emergence and reduced total Striga infection in sorghum and thus resulted in improved sorghum growth and total biomass production. The two millet cultivars produced 5-deoxystrigol and orobanchol and high amounts of orobanchyl acetate. Both millet cultivars, as indicated by
\end{abstract}


low emergence of the parasite in the pot experiment and fewer attachments with arrested development in the rhizotron experiment, displayed high resistance to the Striga ecotype used in this study, making them a suitable alternative crop for sorghum.

Conclusion: The present study demonstrates secretion by sesame and groundnut of active strigolactone germination stimulants into the rhizosphere, which seems to be associated with high suicidal germination and reduction in parasite infection of the host they are intercropped with. This knowledge could help in the selection/breeding/engineering of intercropping cultivars that effectively induce Striga suicidal germination. Millet, in contrast, produces low-efficiency germination stimulants and has mechanical and/or physiological barriers that delay the ingress and/or growth of the examined Striga ecotype. Thus millet could be a suitable alternative crop for fields infested with this Striga ecotype but not an efficient trap or catch crop.

\section{Introduction}

The root parasitic weed, Striga (Striga hermontica (Del.) Benth), remains one of the most serious constraints to production of sorghum ([Sorghum bicolor (L.) Moench]), maize (Zea mays L.), pearl millet (Pennisetum galucum R. BR.) and upland rice (Oryza glaberrima Steud.), the main food crops for millions in sub-Saharan Africa (Scholes et al., 2008a; Atera, 2011; N'Cho, 2014). Infection results in stunted growth, hormonal imbalance, and wilting, significant reductions in biomass and sometimes complete yield loss in sensitive crop varieties (Drennan and El Hiweris, 1979). In sub-Saharan Africa heavy infestation is reported in areas amounting to more than 50 million hectares in more than 24 countries (Emechebe et al., 2004). Further, the parasite is spreading to un-infested areas at worrying rates (Ejeta and Gressel, 2007a). Striga is an obligate parasite and its seeds germinate only in response to a germination stimulant (strigolactone) present in the root exudates of their host and in some cases non-host plant species (Bouwmeester, 2007). After germination, upon contact with the host root, the parasite forms an attachment organ, called haustorium, to attach to its host (Spallek et al., 2013). Upon attachment, the parasite penetrates the host 
root's vascular system and forms a parasitic connection extracting the host's nutrients (Babiker, 2007; Westwood et al., 2010). At this stage (4-7 weeks prior to emergence of the Striga shoot aboveground), much of the damage to the host plant already occurs, making it difficult for farmers to identify the cause of the growth reduction of their crop and control the parasite (Berner et al., 1996; Press and Gurney 2000). After emergence above the ground, the parasite flowers after 4-6 weeks and produces seeds (Bebawi et al., 1984; Mohamed et al., 2007). In sub-Saharan Africa the increasing demand for food has resulted in continuous cultivation of susceptible landraces, with minimum or no application of fertilizers or manure nor fallows. This malpractice has led to devastating levels of infestation, crop failures, and build-up of a large Striga seed bank which may remain viable in the soil for decades (Berner et al., 1996b; Van Ast, et al., 2005; Labrada, 2008).

Striga control approaches used so far - including cultural, chemical, genetic, and biological control measures - are only partially effective and often expensive and/or lacking immediate benefits. Development of sustainable, low-cost and efficient control technologies has for decades been a major and difficult challenge, due to the complexity of the parasite's life cycle and the production of large amounts of seeds with prolonged viability (Scholes et al., 2008; Spallek et al., 2013). Integrating practices that target reduction of existing seed banks, prevention of further seed production and avoiding seed dissemination are needed to successfully contain the problem (Rubiales et al., 2009). Particularly diminishing the Striga seed bank is an important requirement for long term effective Striga management (Joel, et al., 2006; Goldwasser et al., 2013). Since the Striga life cycle begins with the germination of the seeds induced by stimulants present in the root exudate of the host and some non-host species, induction of Striga seed germination in the absence of any host plant, referred to as suicidal germination, has a pronounced potential to reduce the seed bank of Striga in agricultural fields (Babalola et al., 2002). Natural and synthetic compounds such as thiourea, allythiourea, some coumarin-type compounds, cytokinins and ethylene stimulate germination of Striga seeds (Bebawi, 1986; Mangnus et al., 1991, Zwanenburg et al., 2013). Indeed, an almost complete depletion of the Striga 
asiatica seed bank was achieved in the USA with a single application of $1.6 \mathrm{~kg} / \mathrm{ha}$ of ethylene, making it an important part of the Striga eradication project in the USA (Eplee 1981; 1992; Tasker and Westwood 2012). However, ethylene application is very expensive and total eradication of Striga using ethylene in sub Saharan Africa is unlikely to be feasible (Rodenburg et al., 2005). In addition, in east Africa ethylene was shown to be less effective for induction of Striga suicidal germination (Ransom and Njoroge 1991). The concept of suicidal germination has also been approached through the development of synthetic analogues of the strigolactones, with simpler (cheaper) structures and high activity, for example GR24 and Nijmegen 1 (Zwanenburg et al., 2009). These compounds are active at low concentrations and can induce suicidal germination by treating the soil before the crop is sown (Kgosi et al., 2012; Samejima et al., 2016b). However, high costs, the need for large amounts of water and instability of these synthetic strigolactones in alkaline soils have so far precluded the use of synthetic germination stimulants in the field (Sun, et al., 2007; Babiker, 2007).

An alternative strategy to induce suicidal germination is by using non-host trap, or catch crops and intercrops that produce high amounts of germination stimulants, and hence induce massive germination of the parasite, but are resistant to the parasite (trap crops) or harvested before the seeds of the parasite are shed (catch crops) (Sun et al., 2007; Lopez et al., 2009). Hassan et al. (2013) stated that farmers in Africa traditionally intercrop sorghum or maize with Striga non-host legumes to increase crop production and to achieve better returns on fertilizer, pesticide, energy and manpower resources and, in addition, deplete the Striga seed bank. However, for an effective suicidal germination approach, Striga germination in response to the non-host should be as high as possible. Several laboratory experiments have shown that aqueous extracts from roots and shoots of many of these nonhost crop species induce germination of conditioned Striga seeds (Ariga, 1996; Rezig et al., 2016). In vitro germination analysis revealed significant variation in Striga germination activity of the root exudates/and or extracts of intercrop non-host crops (Emechebe et al., 2003). For example root exudates of non-hosts crop such as sunflower stimulated $16.7 \%$, Hibiscus sabdariffa (karkade) 25.5\%, Abelmoschus esculentus (okra) 56.2\%, (dolichos 
beans) Lablab purpureus $40.4 \%$ and cotton (Gossypium spp.) $75 \%$ of germination compared with $48.2 \%$ induced by the root exudate of the highly susceptible sorghum variety Dabar-1 (Traore et al., 2011; Dawoud, et al., 2015). Several authors suggested that variation in the depletion of the Striga seed bank by different trap crops may be accounted for by differences in the stimulation of suicidal germination (Oswald \& Ransom 2001; Khan et al., 2002; Abunyewa \& Padi 2003; Murdoch \& Kunjo 2003). Trap crop species are assumed to contribute significantly to depletion of the parasite seed bank and thus reduce damage inflicted to cereal crops when subsequently grown on the same field (Dawoud, et al., 2015). For example, full-season cultivation of groundnut or soybean followed by a Striga-tolerant cereal was found to reduce the Striga seed bank by up to $50 \%$ in a relatively short time of 2 years (Kabambe, 2008; Oswald \& Ransom 2001; Carsky, 2000). Sauerborn et al., (1999) demonstrated that annual double cropping of trap-crops (soybean, sunflower and cotton) reduced the Striga seed bank by about $30 \%$ each year. Schulz et al., (2003) achieved 50\% seed bank reduction after one-year rotation with soybean and cowpea under farmer-managed conditions. Khan et al., (2007) reported that successive rotations of groundnut, cowpea and soybean with sorghum/maize significantly reduced Striga infestation and increased crop yield. Van Mourik et al., (2011) used a simulation model to show that the use of trap and intercrops would be more effective with respect to Striga seed bank reductions than crop rotation.

The efficiency of a trap crop in controlling Striga infestation will increase if a suitable variety is grown in the appropriate density in the absence of the cereal host (Khan et al., 2002). It has been suggested that the effectivity of an intercrop and/or catch and trap crops can be increased by increasing the production of germination stimulants, which can be achieved through selection or molecular breeding (Sun, et al., 2007). Analysis of varietal differences in the amounts of germination stimulants produced could reduce the time and cost involved in the selection of such optimised trap and intercrops. However, the disadvantage of this approach, may be that other mechanisms that may explain the positive effects of these trap-, catch- and intercrops - such as enhanced seed death, inhibition of 
germination, lowering of soil temperature through shading and physical resistance to the emerging Striga (Kureh et al., 2006) - are ignored.

An alternative solution, when the soil is highly contaminated and the seed bank peaks to a maximum such that cultivation of the preferred crop e.g. sorghum becomes impossible, is crop substitution. Striga populations with specific adaptation to sorghum or millet have long been reported, however it is not always easy to determine if these ecotypes are really different (Vasudeva Rao and Musselman, 1987; Ejeta and Gessel, 2007a). Millet may replace sorghum in locations where the sorghum ecotype of Striga predominates and vice versa (Babiker, 2007). The interpretation of reported Striga resistance in pearl millet is hindered due to the fact that both pearl millet and Striga are genetically highly variable (Kountche et al., 2013). In millet, Ali et al., (2009) reported post-attachment resistance to the Striga sorghum ecotype due to mechanical resistance and/or enzymatic lysis of the parasite during the penetration process. Earlier, Ramaih (1984) suggested that millet and sorghum may produce different germination stimulants, but it is as yet unclear whether that is related to the existence of sorghum and millet-specific Striga ecotypes. Hence, the mechanism underlying the millet resistances to Striga sorghum ecotypes is still poorly understood.

Evaluation of trap-, catch- and intercrops often relies on germination bioassays with their root exudates to estimate their potential field effect. However, root exudates may also contain germination inhibitors, and therefore the amounts of germination stimulants may sometimes be underestimated (Sato et al., 2003). On top of this, there is large genetic variation in Striga, which amongst others, results in different specificity for different germination stimulants. The analysis of strigolactones and their concentration in root exudates of the host and potential trap-, catch-, substitution and intercrops such as groundnut, sesame, millet, cowpea, cotton and soybean could support the right choice of such crops under specific conditions of crop type, Striga ecotype, and climatic and edaphic conditions.

The natural Striga germination stimulants isolated so far are collectively referred to as strigolactones (Ueno et al., 2011). Strigol was the first identified germination stimulant for 
Striga in cotton, a non-host crop (Cook et al., 1966). Later many other strigolactones were identified in root exudates of several Striga host and non-host crops, e.g cowpea, millet, sorghum, rice and maize (Siame et al 1993; Awad et al., 2006; Ueno et al., 2011; Charnikhova et al., 2017). However, the strigolactones in the root exudates of several leguminous non-host crops often used to suppress Striga infestation in Africa have not been identified.

All plant species typically produce mixtures of various strigolactones and these mixtures differ between species (Cardoso et al., 2014). The different strigolactones exhibit different stimulatory activity that can be over 100-fold different (Yoneyama, et al., 2009 Cardoso et al., 2014; Mohemed et al., submitted. Strigolactones (SLs) are classified into two structurally distinct groups: canonical and non-canonical SLs. Canonical SLs contain the $\mathrm{ABCD}$ ring system, and non-canonical SLs lack the $\mathrm{A}, \mathrm{B}$, or $\mathrm{C}$ ring but have the enol ether$\mathrm{D}$ ring moiety which is essential for biological activities. The D-ring and the enol-ether bridge are essential for induction of parasitic plant seed germination (Zwanenburg and Pospísil, 2013). Based on the stereochemistry of the C-ring, canonical strigolactones are divided into two groups with $\alpha$ - (orobanchol-type) or $\beta$-oriented C-ring (strigol-type) (Xie et al., 2016) (Fig. 1). 

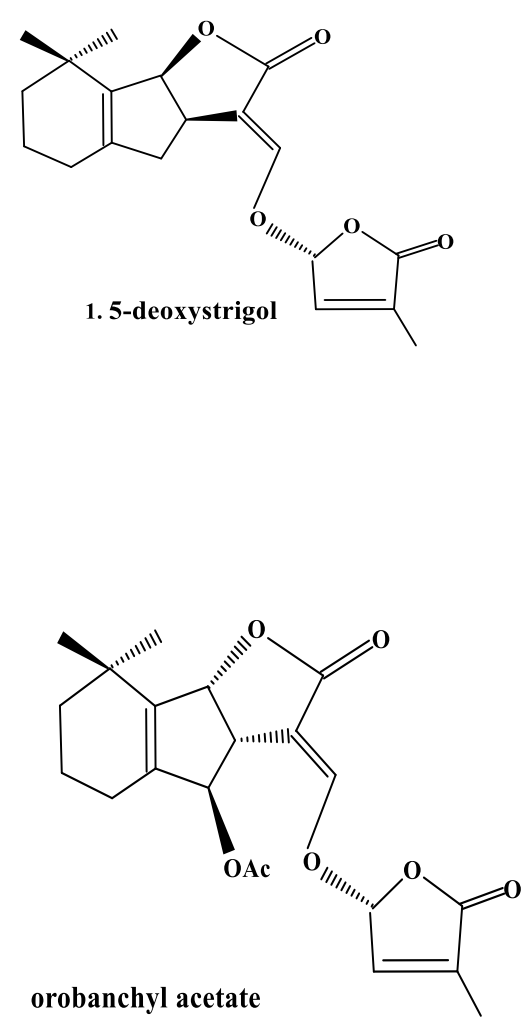
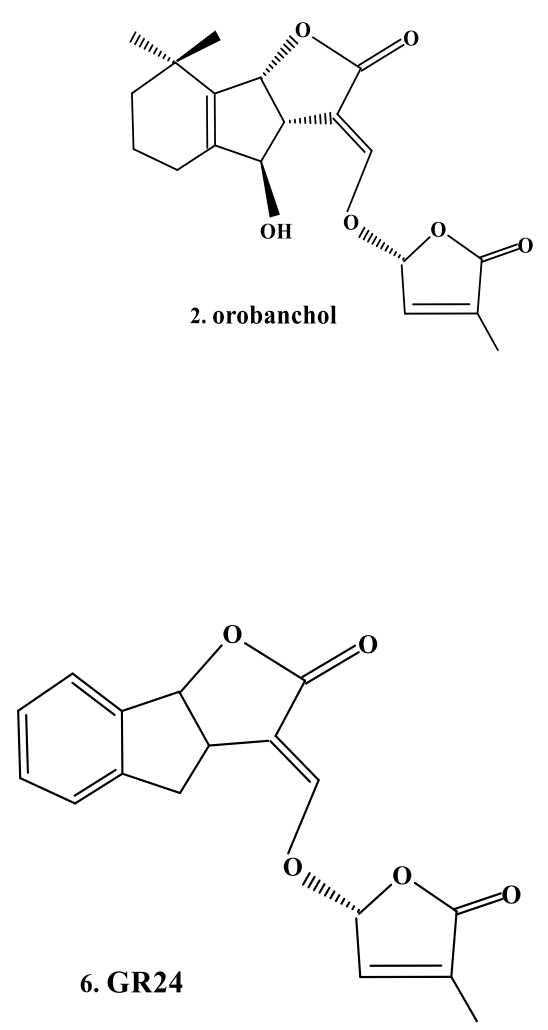

Fig. 1 Chemical structure of the natural Striga germination stimulants 5-deoxystrigol, orbanchol, orobanchyl acetate and the synthetic strigolactone analogue GR24 that are present in the root exudates of sesame, ground nuts pearl millets or sorghum genotypes or were tested in Striga germination bioassays.

Striga, sorghum ecotype, is more sensitive to strigol-type strigolactones and root exudates that contain strigolactones such as strigol, sorgolactone, sorgomol and 5-deoxystrigol often trigger significant Striga seed germination (Sugimoto et al., 1998; Nomura et al., 2013; Mohemed et al., 2016). In addition to the canonical strigolactones, non-canonical strigolactones (without the $\mathrm{ABC}$ scaffold) are being identified, e.g. in maize (Charnikhova et al., 2017). One of these recently discovered strigolactones, zealactone, has considerable activity in the induction of germination of Striga (Charnikhova et al., 2017). Leguminous crops characterized so far, e.g. cowpea, produce mainly orobanchol-type strigolactones (Ueno et al., 2011). Intriguingly, orobanchol production was shown to impart resistance to Striga in sorghum (Mohemed et al., 2016; Gobena et al., 2017) 
In this study, therefore, we analysed the composition and concentration of strigolactones in the root exudates of sesame, groundnut and pearl millet. In parallel, we assessed the germination stimulatory activity of these root exudates and Striga infection of a host (sorghum) and a substitution crop (millet) as well as infection of sorghum in the presence of an intercrop. Using these data we tried to delineate the role of strigolactones in determining the efficiency of crop substitution and intercropping for Striga management.

\section{Materials and Methods}

\section{Germplasm and chemicals}

Seeds of different genotypes of sesame, groundnut, pearl millet and sorghum were provided by the Agricultural Research Corporation, Sudan. [Striga hermonthica (Del.) Benth.] seeds were collected in 2009/2010, from under sorghum grown, at three different sites in Sudan: Abu Naama, Gezira and Gadarif. Striga seed germinability was about 60$70 \%$. Strigolactone standards were provided by professor Koichi Yoneyama (Weed Science Center, Utsunomiya University, Japan) and professor Tadao Asami (Applied Biological Chemistry the University of Tokyo, Japan).

\section{Striga infection experiments}

Pearl millet and sorghum cultivars were grown in pots in a glasshouse at Wageningen University, the Netherlands, to examine genotypic responses to Striga infection. White and Red millet cultivars were infected with Striga collected from under sorghum grown in Gadarif in eastern Sudan, where the latter is the main crop. Plants were grown in 18L pots $(1 \times \mathrm{w} \times \mathrm{h}=0.25 \mathrm{~m} \times 0.25 \mathrm{~m} \times 0.30 \mathrm{~m})$ containing a mixture of sand and arable soil, collected from the top layer $(0-0.25 \mathrm{~m})$ of an arable field near Wageningen. Striga seeds $(25 \mathrm{mg}$ ) were added and mixed with the soil in each pot. Five seeds of each cultivar were sown in the middle of the respective pot and the seedlings were thinned to one 5 days after emergence. For intercropping experiments, intercrop species and sorghum genotypes were grown in pots using soil infested with Striga seeds, as described above. Sesame and groundnut were sown one week earlier than sorghum and were thinned to 5 seedlings per 
pot 10 days after emergence while sorghum was thinned to one seedling as described above. All infection experiments were carried out in a temperature-controlled glasshouse, $28^{\circ} \mathrm{C} / 25^{\circ} \mathrm{C}$ light $12 /$ dark 12 glass greenhouse, with $50-60 \%$ relative humidity. Supplemental light was provided by high-pressure sodium lamps (400 W SON-T, Agro Philips lamps), which automatically switched on during day time when photosynthetically active radiation outside the greenhouse dropped below $910 \mu \mathrm{mol}$ photons $\mathrm{m}^{-2} \mathrm{~s}^{-1}$. Black screens were used to reduce the day length to $12 \mathrm{~h}$ throughout the entire growing period. Half strength modified Hoagland's nutrient solution was applied in the first week $(250 \mathrm{~mL}$ at $48 \mathrm{~h}$ intervals). For the remainder of the experimental period a nutrient solution with $20 \% \mathrm{P}$ was applied to stimulate strigolactone exudation $(250 \mathrm{~mL}$ to each pot at $48 \mathrm{~h}$ intervals). Treatments were set up in a randomized complete block design with four replications.

To estimate the number of days to first Striga emergence data collection on Striga infection started when Striga emergence was observed for the first time on the susceptible genotype Gadam Elhamam, about two weeks after sowing. From that date on the total number of emerged Striga seedlings per pot per genotype were recorded every 3 days until Striga had emerged in all 20 genotypes. Then the number of Striga plants was counted regularly at weekly intervals for 10 weeks. The total number of Striga plants per host plant was determined after harvesting of each genotype at maturity by root washing and counting of attached Striga tubercles and plants.

\section{Striga infection in a rhizotron system}

Sesame, groundnut, pearl millet and sorghum seeds were pre-germinated in a vermiculite filled plastic tray. After 3 days, seedling roots were washed to clean vermiculate and transferred to a tube filled with distilled water to spread the roots, 2 days later the seedlings were transferred to an observation chamber (rhizotron), consisting of a $25 \times 25 \mathrm{~cm}$ Perspex square Petri dish, packed with rock wool and lined with vermiculite and on the top side a $100 \mu \mathrm{m}$ nylon mesh was placed. Holes were made on the top side and bottoms of the Petri dishes to allow shoot growth and excess nutrient draining, as described by Gurney et al., 
(2006). The rhizotrons were covered with aluminum foil and supplied with $25 \mathrm{ml}$ of Hoagland's nutrient solution containing $\mathrm{NH}_{4} \mathrm{NO}_{3}(5.6 \mathrm{mM}), \mathrm{K}_{2} \mathrm{HPO}_{4}(0.4 \mathrm{mM}), \mathrm{MgSO}_{4}$ (0.8 mM), $\mathrm{FeSO}_{4}(0.18 \mathrm{mM}), \mathrm{CaCl}_{2}(1.6 \mathrm{mM}), \mathrm{K}_{2} \mathrm{SO}_{4}(0.8 \mathrm{mM}), \mathrm{MnCl}_{2}(0.0045 \mathrm{mM})$, $\mathrm{CuSO}_{4}(0.0003 \mathrm{mM}), \mathrm{ZnCl}_{2}(0.0015 \mathrm{mM})$ and $\mathrm{Na}_{2} \mathrm{MoO}_{4}(0.0001 \mathrm{mM})$ twice a week. The plants were maintained in a growth chamber in a 12-h photoperiod. Day and night temperatures were set at 28 and $24^{\circ} \mathrm{C}$, respectively, and the relative humidity was set at $60 \%$. After 7 days, sorghum seedlings in the system were infected with pre-germinated Striga seeds (pre-treated with GR24 for 48h) to exclude effects of different germination rates caused by different strigolactone levels produced by different genotypes. GR24treated Striga seeds were placed next to the roots in the rhizotron chambers using a wet soft paint brush. Attachment, penetration and development were observed from 3 to 10 days after the inoculation, using a binocular stereo- microscope. A Striga seedling was considered to be attached when it formed a primary haustorium that actually penetrated into the host root cortex. Upon penetration, the host root surface was clearly split around the site of infection (Lane et al., 1991). Attachment percentages were based on the total number of germinated Striga seeds inoculated onto the roots (about 100). A Striga seedling was considered to be successfully connected with the host vascular system when the cotyledons emerged from the seed coat. Parasite development was assessed for a period of 20-28 days after inoculation in four replicates.

\section{Analysis of strigolactones in root exudates}

The root exudates of all crop species and cultivars were analysed for strigolactones. For collection of root exudates, sesame, groundnut, pearl millet and sorghum genotypes were grown in a climate room in Wageningen, the Netherlands. The experiment was laid out as a complete randomized block design with four replicates per genotype. Germinated sesame, groundnut, pearl millet and sorghum seeds were planted in a $3 \mathrm{~L}$ plastic pot filled with $1.5 \mathrm{~L}$ sand. After one week, the seedlings were thinned to five per pot. Half strength modified Hoagland's nutrient solution containing $\mathrm{NH}_{4} \mathrm{NO}_{3}(5.6 \mathrm{mM}), \mathrm{K}_{2} \mathrm{HPO}_{4}(0.4 \mathrm{mM})$, $\mathrm{MgSO}_{4}(0.8 \mathrm{mM}), \mathrm{FeSO}_{4}(0.18 \mathrm{mM}), \mathrm{CaCl}_{2}(1.6 \mathrm{mM}), \mathrm{K}_{2} \mathrm{SO}_{4}(0.8 \mathrm{mM}), \mathrm{MnCl}_{2}(0.0045$ 
$\mathrm{mM}), \mathrm{CuSO}_{4}(0.0003 \mathrm{mM}), \mathrm{ZnCl}_{2}(0.0015 \mathrm{mM})$ and $\mathrm{Na}_{2} \mathrm{MoO}_{4}(0.0001 \mathrm{mM})$ was applied to each pot (500 $\mathrm{mL}$ at 48 hours intervals). The plants were grown in a climate room with artificial lighting at $450 \mu \mathrm{mol} \mathrm{m} \mathrm{s}^{-2}$ and controlled conditions $\left(28^{\circ} \mathrm{C}\right.$ (D) $10 \mathrm{~h}$ and $25^{\circ} \mathrm{C}$ (N) $14 \mathrm{~h}$ at $70 \%$ relative humidity) for four weeks. In the 5 th week, phosphorus deficiency was created in each pot to increase strigolactone production. Here $3 \mathrm{~L}$ phosphorus deficient nutrient solution (half strength Hoagland's nutrient solution minus phosphate) was added to each pot and allowed to drain freely through the holes in the bottom of the pot to remove phosphorus from the sand. The plants were kept under P deficiency for one week and subsequently drained again with $3 \mathrm{~L}$ of phosphorus free nutrient solution to remove any accumulated strigolactones. Finally, 48 hours later, root exudates were collected in $1 \mathrm{~L}$ plastic bottle by passing $1.5 \mathrm{~L}$ nutrient solution without phosphate through each pot. The collected root exudates were then run through an SPE C18 column (500 mg) and strigolactones were eluted with $6 \mathrm{~mL}$ acetone. For further purification $4 \mathrm{~mL}$ were taken up in hexane, loaded on pre-equilibrated Silica gel Grace Pure SPE (200 mg/3ml) columns and eluted with $2 \mathrm{~mL}$ hexane:ethyl acetate (1:9). The solvent was evaporated and the residue dissolved in $200 \mu \mathrm{l}$ of $25 \%$ acetonitrile in water and filtered through Minisart SRP4 $0.45 \mu \mathrm{m}$ filters (Sartorius, Germany) before LC-MS/MS analysis.

The samples were analysed on a Waters Xevo triple quadrupole tandem mass spectrometer (Waters, Milford, MA, USA) equipped with an electrospray ionization source coupled to an Acquity UPLC system (Waters, USA) as previously described by Lopez-Raez, et al., (2008). Data acquisition and analysis were performed using Mass Lynx 4.1 (TargetLynx) (Waters). The strigolactones, 5-deoxystrigol, sorghomol, orobanchol, sorgolactone-like, sorgomol-like, solonacol-like, didehydro-orobanchol isomer like and orobanchyl acetate were (tentatively) identified and quantified. Because 5-deoxystrigol, sorgomol, orobanchol, and orobanchyl acetate have not been reported before in sesame and groundnuts, their identification was carefully confirmed - by MS/MS and co-injection using authentic standards as described before by Lopez-Raez, et al., (2008). For semiquantification of unknowns, sorgolactone-like, sorgomol-like, solonacol-like and didehydro-orobanchol isomer like the peak area was used. 


\section{Striga germination}

The root exudates of all crop species and genotypes were assessed for germination stimulant activity in a Striga seed germination bioassay as reported before (Matusova et al., 2004). For preconditioning, surface-sterilization of Striga seeds was done using $25 \mathrm{~mL}$ ( $2 \%$ ) sodium hypochlorite with $0.4 \%$ Tween-20 for 5 minutes. Subsequently, seeds were thoroughly rinsed three times at 10 min intervals using sterile demineralized water through a Buchner funnel. The sterile seeds were air dried for sixty minutes. Approximately 50 to100 seeds were then evenly spread on $9 \mathrm{~mm}$ diameter glass fiber filter paper discs (Sartorius, Germany). These discs were placed in $9 \mathrm{~cm}$ diameter Petri dishes (12 discs per dish) on filter paper (Whatman, UK) moistened with $3 \mathrm{~mL}$ demineralized water. The Petri dishes were sealed with parafilm, wrapped in aluminium foil and placed in an incubator at $30^{\circ} \mathrm{C}$ for 10 days. After 10 days, the discs with preconditioned seeds were allowed to dry for $50 \mathrm{~min}$ in a laminar flow cabinet to evaporate surplus moisture. The discs were then placed in another Petri dish (six per dish) containing a filter paper ring (outer diameter 9 $\mathrm{cm}$, inner diameter $8 \mathrm{~cm}$ ) moistened with $0.9 \mathrm{~mL}$ water. The root exudates to be tested were applied $(50 \mu \mathrm{L})$ to triplicate discs after replacement of the acetone in the samples by distilled water through vacuum centrifugation. GR24 $(0.2 \mu \mathrm{M})$ was used as a positive control and distilled water as a negative control in each germination assays. Seeds were again incubated at $30^{\circ} \mathrm{C}$ in darkness for 48 hours and germination (seeds with radicle protruding through the seed coat) was scored using a binocular microscope (Matusova et al., 2004).

\section{Statistical analysis}

The statistical package SAS (version 15) was used for ANOVA and Pearson's correlation analyses. Duncan's honest significant difference test was subsequently performed to establish the significance of differences. The relationship between various strigolactones and number of emerged Striga, Striga biomass and in vitro Striga germination was analysed by correlation analysis and stepwise regression using IBM SPSS Statistics 20. OrignPro 9 (64-bit) was used for PCA analysis. To meet the assumptions of the analysis of variance, 
data involving strigolactone peak areas and number of emerged Striga were subjected to logarithmic transformation prior to analysis. The peak areas of 5-deoxystrigol, orobanchol, sorgolactone-like, solonacol-like and didehydro-orobanchol were used in PCA analysis to visualize the relationship between strigolactone composition, the germination inducing activity of root exudates and the number of Striga plants per pot. 


\section{Results}

\section{Strigolactone production}

Root exudates of sesame, groundnut, pearl millet and sorghum cultivars were collected and purified using C18 and Silica gel Grace Pure SPE $(200 \mathrm{mg} / 3 \mathrm{ml})$ column and eluted with 2 $\mathrm{mL}$ hexane: ethyl acetate (1:9). The solvents were evaporated and the residue was dissolved in $200 \mu 1$ of $25 \%$ aqueous acetonitrile and filtered through Minisart SRP4 $0.45 \mu \mathrm{m}$ filters (Sartorius, Germany) prior to LC-MS/MS analysis. Then MRM-LC-MS/MS analysis was performed to identity and quantify strigolactones. In the UPLC-MS/MS (MRM) chromatograms of the root exudates of sesame, groundnut and pearl millet intense signals were detected for the known strigolactones 5-deoxystrigol, orobanchol and orobanchyl acetate in the different MRM channels. 5-Deoxystrigol was detected at RT $8.02 \mathrm{~min}$ in MRM channels $\mathrm{m} / \mathrm{z} 331>216,331>334$ and 331>97, orobanchol was detected at RT 4.61 min in MRM channels $m / z 347>233,347>205$ and 347>97 and orobanchyl acetate was detected as major compound at Rt $7.2 \mathrm{~min}$ in MRM channel $\mathrm{m} / \mathrm{z} 347>233$. Four strigolactone-like compounds which have not been previously reported in sorghum or the other crop species were tentatively named solanacol-like, detected at RT 3.6 min in MRM channels $\mathrm{m} / \mathrm{z} 343>183$ and 343>96, didehydro-orobanchol isomer like, detected at RT 4.2 min in MRM channels $m / z 345>203$ and 345>97, sorgolactone-like, detected at $7.6 \mathrm{~min}$ in MRM channel $\mathrm{m} / \mathrm{z}(317>97)$ and sorgomol-like at Rt $4.9 \mathrm{~min}$ in MRM channels $\mathrm{m} / \mathrm{z}$ $317>97$ and $347>317$ (Table S2).

\section{Varietal differences in strigolactone profiles}

Strong qualitative and quantitative variation in strigolactone production was observed between crop species as well as among cultivars of the same plant species (Fig. 2A-F). The groundnut cultivar Gadarif produced the highest amounts of 5-deoxystrigol and also produced low amounts of orobanchol, sorgolactone-like and didehydro-orobanchol isomer-like but no solanacol-like strigolactones (Fig. 2A). The groundnut cultivar Blue nile produced high amounts of sorgolactone-like, intermediate amounts of 5-deoxystrigol, low amounts of orobanchol and didehydro-orobanchol isomer-like and also no solanacol-like 
(Fig. 2B). The sesame cultivar Abu Sofa showed also high production of 5-deoxystrigol, intermediate production of orobanchol, solanacol-like and didehydro-orobanchol isomer like and low sorgolactone-like strigolactones (Fig. 2C).

While the other sesame cultivar Abu Naama was the lowest 5-deoxystrigol producer but it produced relatively higher levels of orobanchol, didehydro-orobanchol isomer-like, sorgolactone-like and solanacol-like strigolactones (Fig. 2D). The sorghum cultivar Gadam Elhamam produced very high amounts of 5-deoxystrigol and relatively low amounts of sorgomol and orobanchol but no traces of didehydro-like and solanacol-like strigolactones were detected. The substitution crop, pearl millet cultivars, showed quite a distinct strigolactone profile in comparison to sorghum (Fig. 2E-G). Pearl millet White cultivar produced low amounts of 5-deoxystrigol, orobanchol, sorgomol and sorgomol-like strigolactone and high amounts of orobanchyl acetate, wehereas the Red cultivar, produced low amounts of 5-deoxystrigol and sorgomol-like strigolactone and high amount of orobanchyl acetate, but no orobanchol or sorgomol was detected (Fig. 2F and G). 


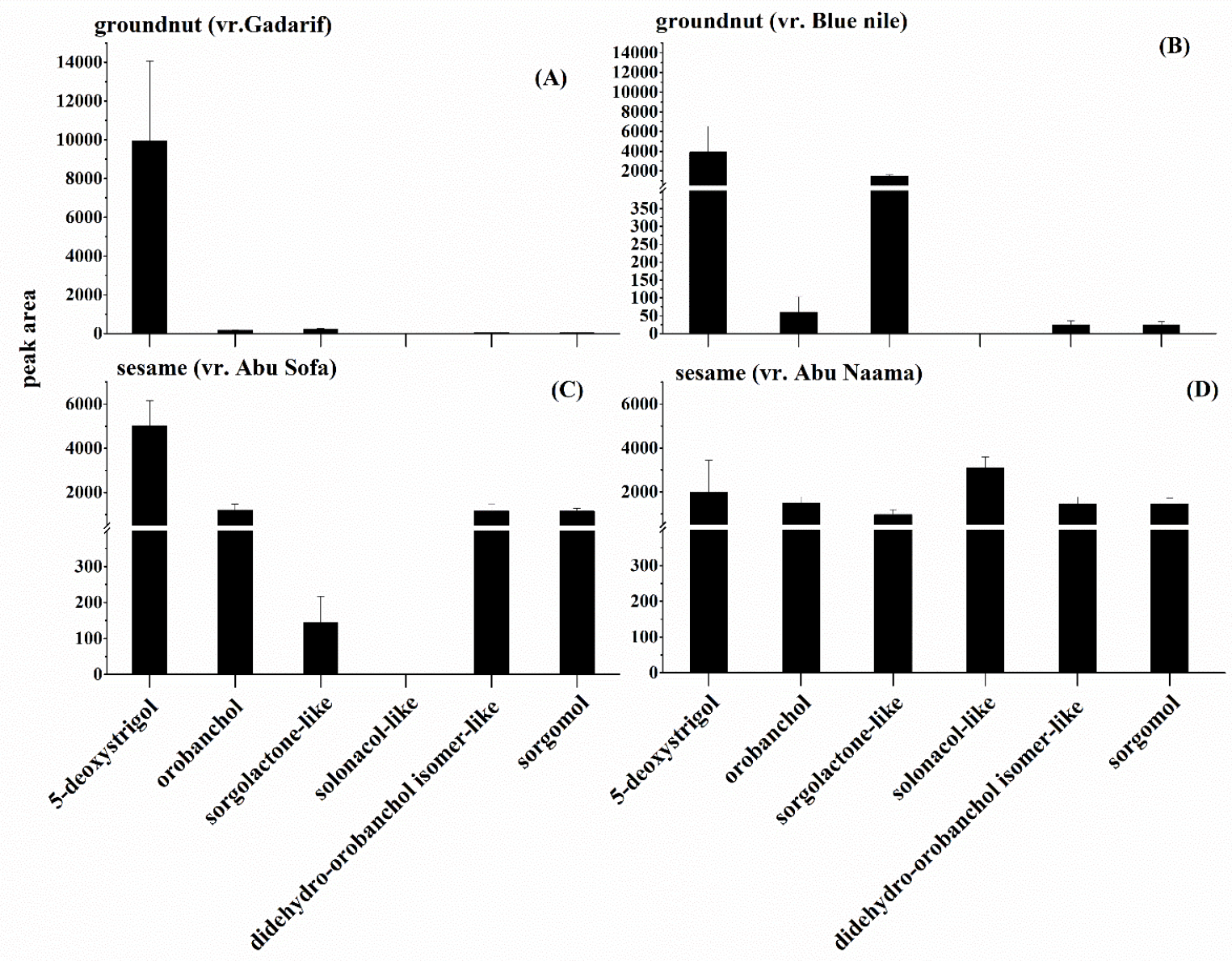




\section{Sorghum (vr.GadamElhamam)}

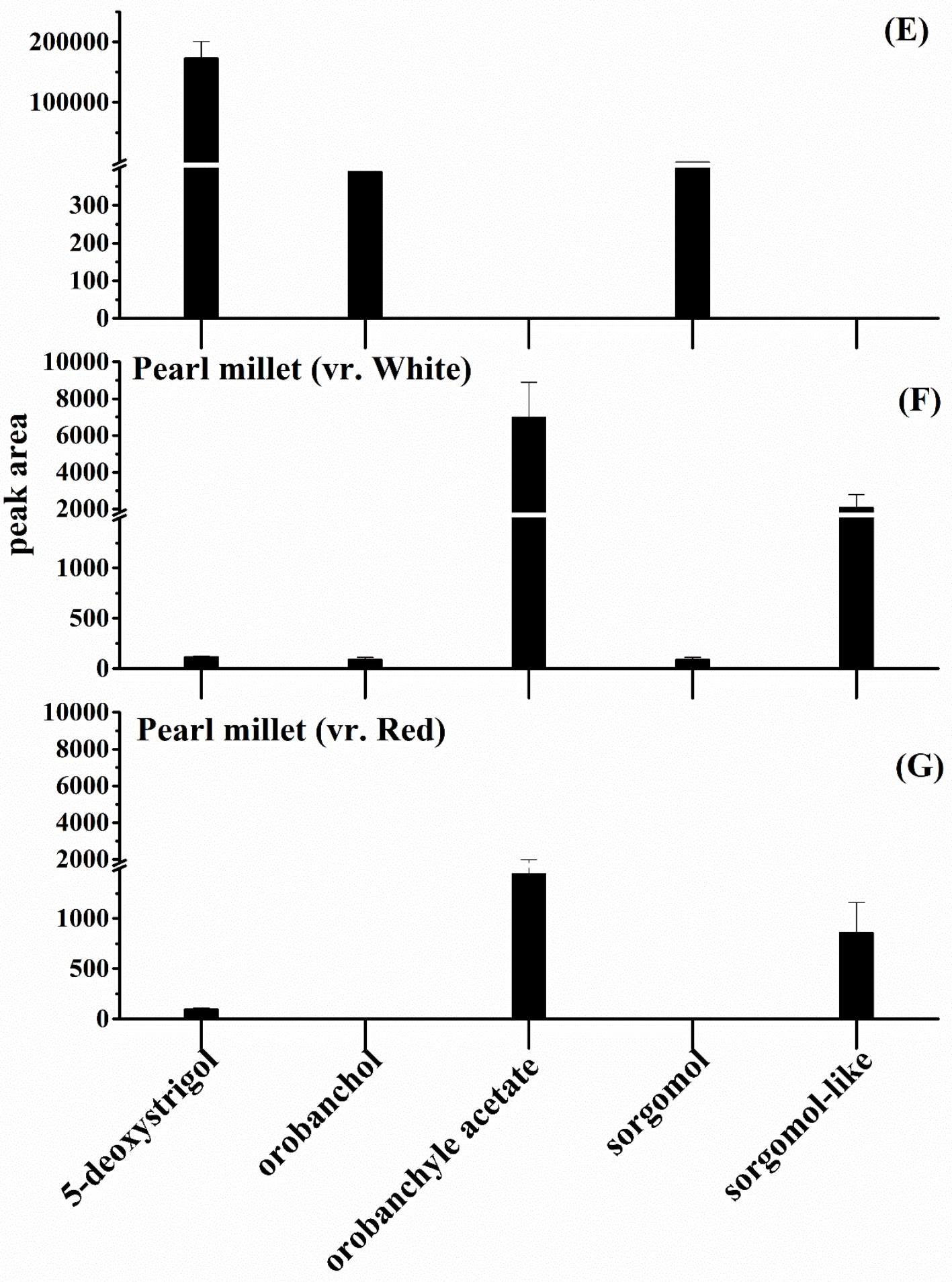


Fig. 2 Strigolactones 5-deoxystrigol, orobanchol, orobanchyl acetate, sorgolactone-like, solonacol-like and didehydro-orobanchol isomer-like (peak area) in root exudates of sesame, groundnut, sorghum and millet, analysed using liquid chromatography coupled with tandem mass spectrometry (LC-MS/MS). Bars represent means \pm standard error $(n=4)$.

\section{Striga seed germination}

To determine the germination stimulation activity of the root exudates, the $\mathrm{C}-18$ purified 100-fold diluted root exudates were used in a Striga germination bioassay. The synthetic Striga germination stimulant GR24 and exudates of the sorghum cultivars Gadam Elhamam were included as positive controls. The two sesame cultivars (Abu Naama and Abu Sofa) and the groundnut cultivar Gadarif induced 30-35\% germination (Fig. 3). The two millet cultivars and the groundnut cv Blue nile induced 24-25\% germination. GR24 and the sorghum cultivar, on the other hand induced 24 and $43 \%$ germination, respectively (Fig. 3). 


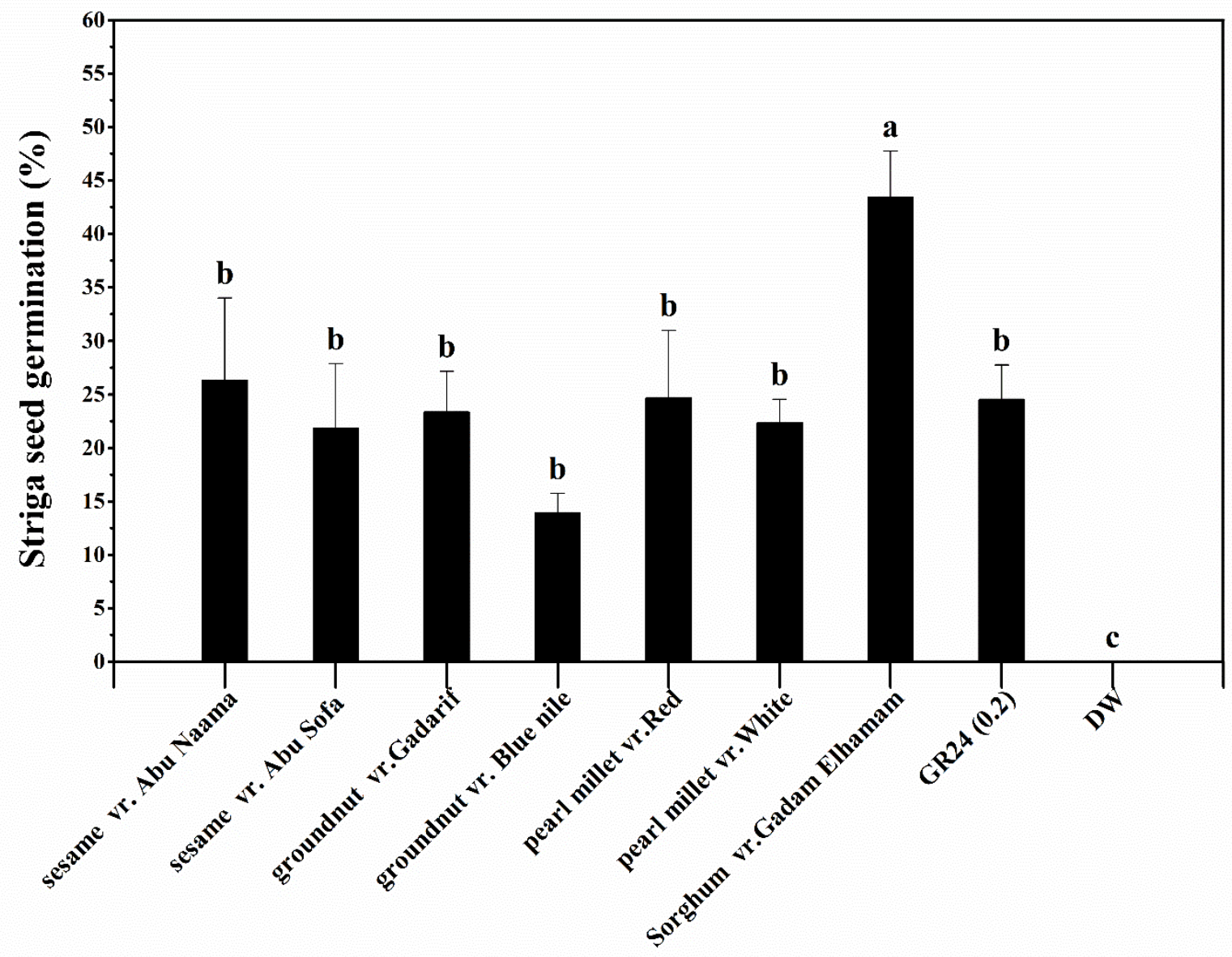

Fig. 3 Germination of pre-conditioned Striga seeds as induced by root exudates collected from different sesame, groundnuts, and pearl millet cultivars and sorghum cv Gadam Elhamam s. Bars represents means \pm standard error $(\mathrm{n}=5)$. Least significant differences of means at $P=0.05$ by ANOVA test. Letters indicate the different significance groups after Duncans' pairwise comparisons $(P<0.05)$. DW= distilled water.

\section{Striga infection}

To determine to what extent the strigolactone profiles in root exudates and associated germination activity impact the capacity of sesame and groundnut to function as intercrop and millet as substitution crop we assessed Striga infection in sorghum intercropped with sesame and/or groundnut and we compared the Striga infection level in sorghum and. Striga emergence in sorghum intercropped with sesame Abu Naama and sesame Abu Sofa was delayed by up to 28 days after sowing (DAS) compared to the sole sorghum treatment 
(Fig. 4A). In sorghum intercropped with groundnut, Striga emergence was delayed by up to 21 days compared with sole sorghum (Fig. 4B). The mean number of emerged Striga plants per pot 50 days after sowing (DAS) in sorghum intercropped with either sesame or groundnut was significantly $(\mathrm{P}<0.001)$ reduced compared with the emerged Striga plants on sole sorghum. Emerged Striga plants per pot were reduced by 75 to $50 \%$ in sorghum intercropped with sesame and groundnut, respectively (Fig. 4C). Intercropping sorghum with sesame cultivar Abu Naama showed $(\mathrm{P}<0.01)$ the maximum reduction in the number of emerged Striga plants per pot $(<10$ Striga plants per pot). The two groundnut cultivars showed relatively similar reduction in the number of emerged Striga plants per pot. 


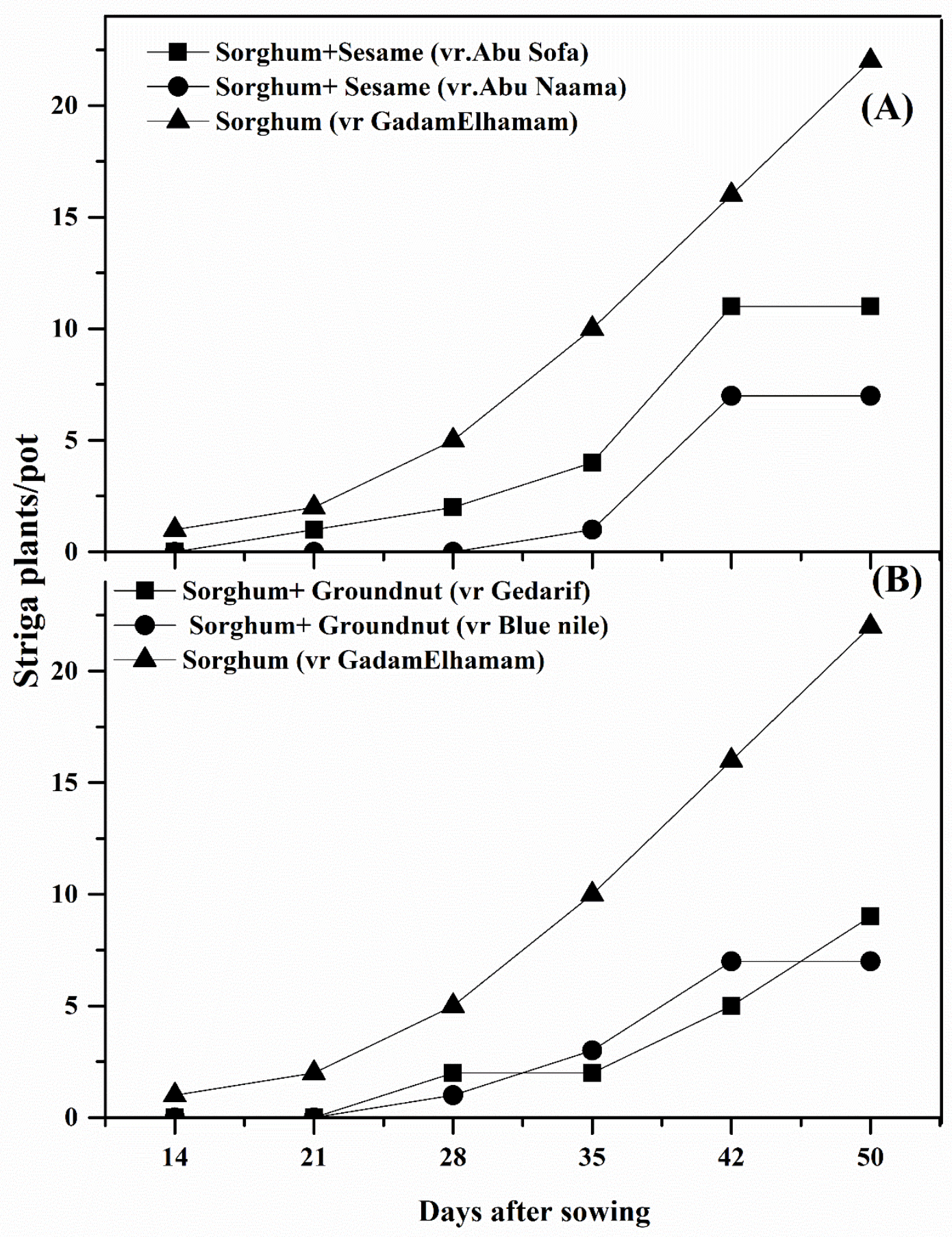

Fig. 4 A-B. Time course of Striga emergence in the greenhouse pot experiment in inter-crop sesame Abu Naama, Sofa and groundnut Gadarif and sorghum genotype Gadam Elhamam. Lines represent mean total emergence of Striga hermonthica plants per pot during a period of 50 days (4 replicates). 


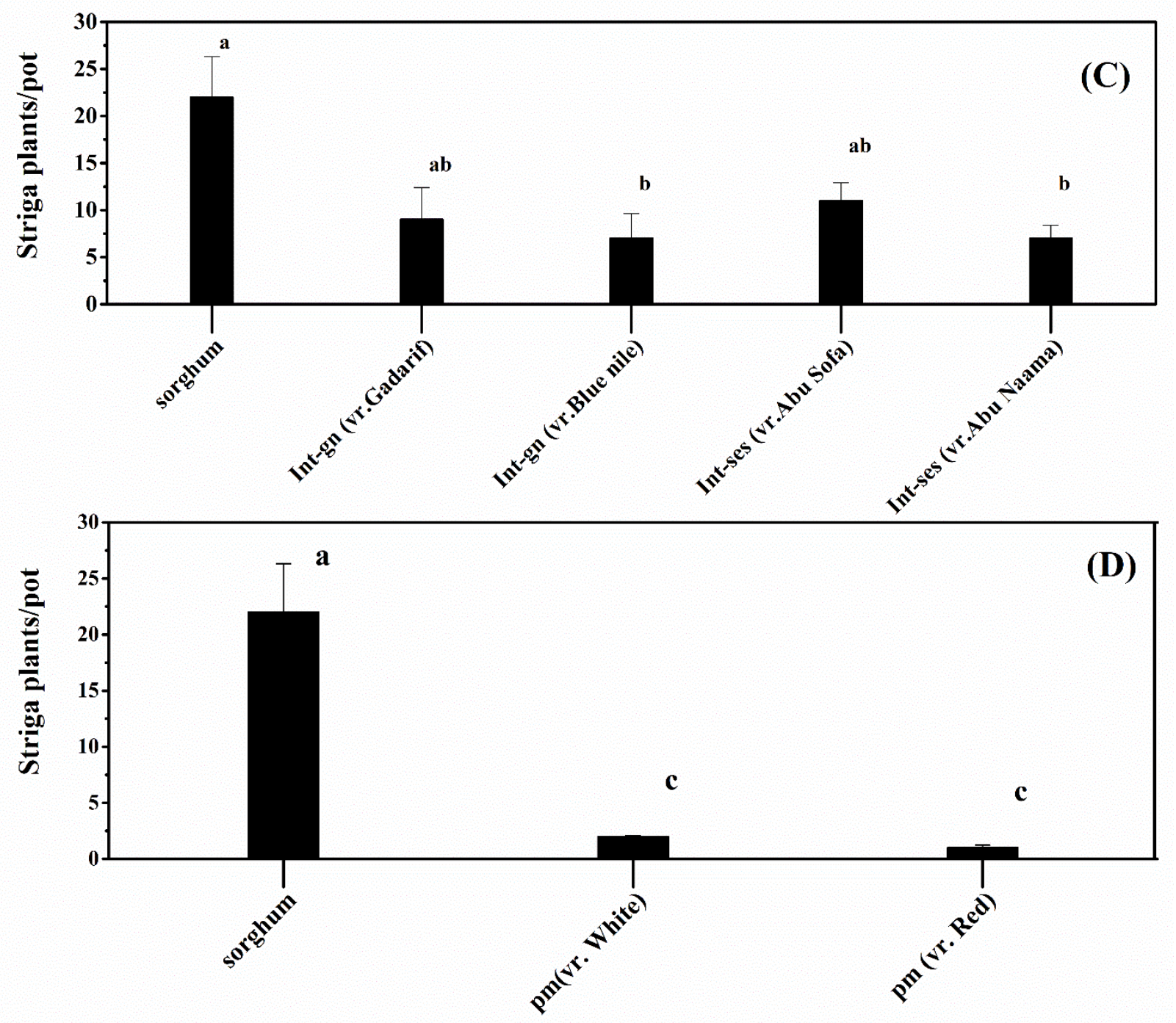

Fig. 4C-D. Effects of intercropping of sorghum with (gn), groundnuts and (ses), sesame on Striga emergence at harvest. (ses) sesame Abu Naama, (ses) Abu Sofa and (gn) groundnuts Gadarif and (gn) Blue nile cultivars and in sole sorghum cultivar Gadam Elhamam, D) Comparison of Striga emergence on sorghum cultivar Gadam Elhamam and pearl millet (pm) cultivars White and Red. Bars represent means of total emerged Striga plants per pot at harvest \pm standard error $(n=4)$. The significance of a treatment effect was determined by a one-way ANOVA for all genotypes and species and the letters indicate the different significance groups after Duncans' pairwise comparisons $(P<0.05)$. 
On the pearl millet cultivars, White and Red, Striga displayed the lowest emergence, 2-3 Striga plants per pot, in comparison to sorghum which showed 29 Striga plants/pot (Fig.4D). The cv White was also tested with two other Striga ecotypes, collected from under sorghum at Gadarif and Gezira. Pearl millet displayed a significantly lower infection by all 3 Striga ecotypes ( $<5$ plants per pot), 50 days after sowing than sorghum cv Gadam Elhamam with around 29 Striga plants per pot (Fig. 4D,E). On the sorghum cultivar, the Striga ecotype Abu Naama showed the highest emergence ( 29 plants/per pot) followed by Gezira and Gadarif ecotypes, with 26 and 20 plants/pot, respectively (Fig. 4E). This suggests that pearl millet exhibits a broad-spectrum resistance response to the sorghum Striga ecotypes, while sorghum Gadam Elhamam exhibited high susceptibility to all three Striga ecotypes, even though they were collected from different locations. 


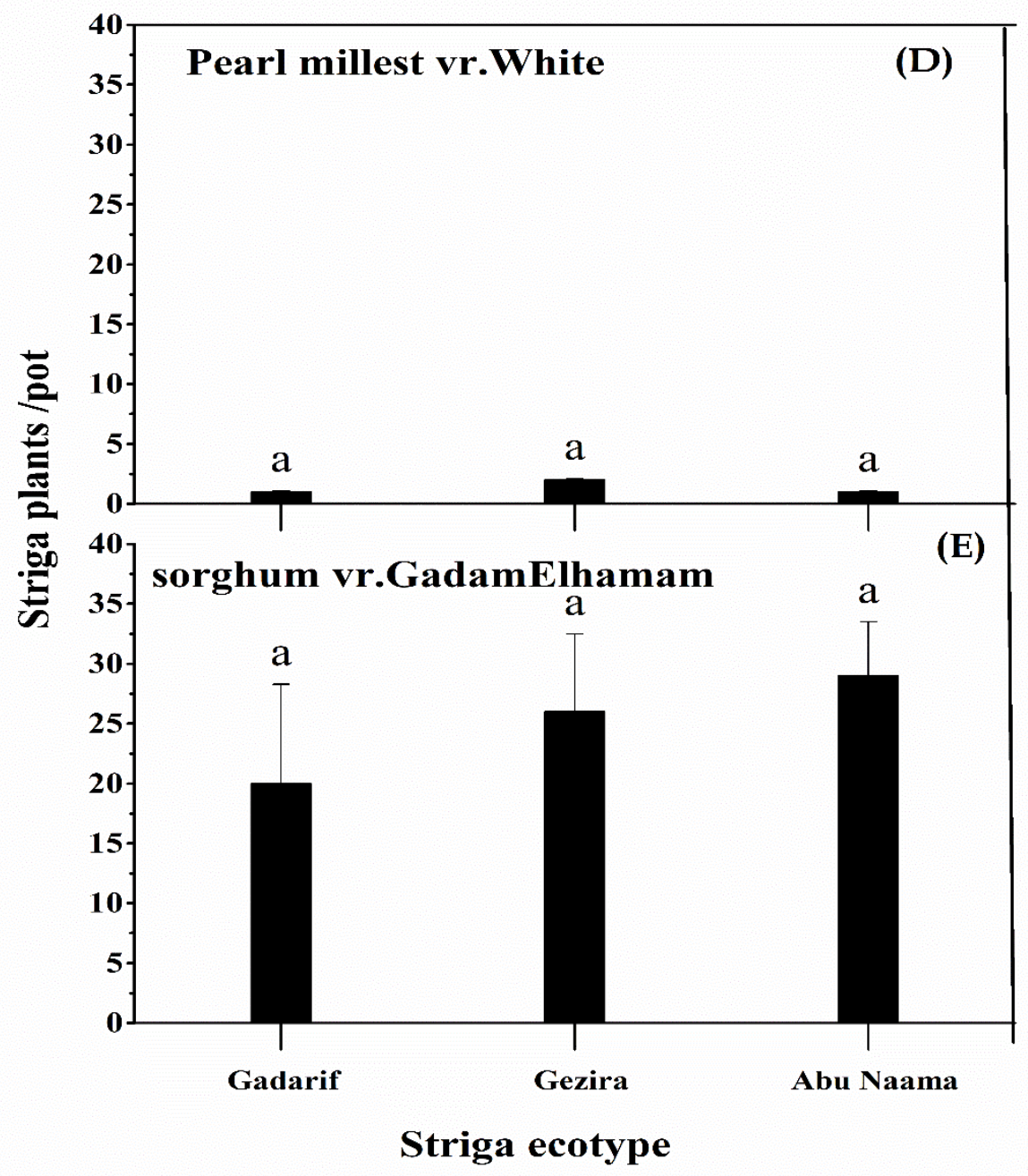

Fig. 4 D-E. Comparison between in the 3 Striga ecotypes emergence on sorghum and pearl millet cultivar White and sorghum genotype Gadam Elhamam. Bars represent means of total emerged Striga plants per pot at harvest \pm standard error $(n=4)$. The significance of a treatment effect was determined by a one-way ANOVA for all genotypes and species and the letters indicate the different significance groups after Duncans' pairwise comparisons $(P<0.05)$

\section{Relationship between strigolactone production and Striga infection}

The peak areas of 5-deoxystrigol, sorgolactone-like, orobanchol, solanacol-like and didehydro-orobanchol were used in PCA analysis to visualize the relationship between the strigolactones present in the root exudates of non-host crop species and their germination inducing activity and number of Striga plants per pot (Fig. 5). The first two principal 
components had an Eigenvalue higher than one and explained $63 \%$ of the variation in strigolactone content (Table 1). The first principle component, PC1, explained $36 \%$ of the variation, with positive loadings for 5-deoxystrigol, germination stimulating activity, orobanchol, solanacol-like and didehydro-orobanchol and a negative loading for sorgolactone-like and number of Striga plants per pot (Table 1). The second principle component (PC2) explained $26 \%$ of the total variation with positive loadings for sorgolactone-like and Striga numbers per pot, orobanchol, solanacol-like and didehydroorobanchol and a high negative loading for 5-deoxystrigol and germination stimulating activity (Table 1). In Fig. 5 according to the directions and angles of the vectors, 5 deoxystrigol exhibited a positive correlation with the germination stimulating activity and a negative correlation with Striga plants per pot while sorgolactone-like showed an inverse correlation with germination stimulating activity and positive correlation with Striga plants per pot (Fig. 5). Orobanchol, solanacol-like and didehydro-orobanchol isomer-like showed no or just a weak correlation with germination stimulating activity and Striga plants per pot (Fig. 5).

Table 1 Principal Component (PCS) analysis of strigolactones Striga seed germination and emergences per pot, showing Eigenvalues, variances and traits contribution to the total variations explained with the first two principal component axes.

\begin{tabular}{lll} 
Total variance (\%) & PC1 & PC2 \\
\hline Eigenvalue & 2.53 & 1.89 \\
Individual & 36.16 & 27.10 \\
Cumulative (\%) & 36.16 & 63.26 \\
\hline Descriptor & trait loading & \\
& PC1 & PC2 \\
\hline 5-deoxystrigol & 0.12 & -0.41 \\
orobanchol & 0.57 & 0.03 \\
sorgolactone-like & -0.38 & 0.38 \\
solanacol-like & 0.47 & 0.34 \\
didehydro-orobanchol isomer like & 0.40 & 0.47 \\
Germination \% & 0.30 & -0.37 \\
Striga plants per pot & -0.13 & 0.44 \\
\hline
\end{tabular}




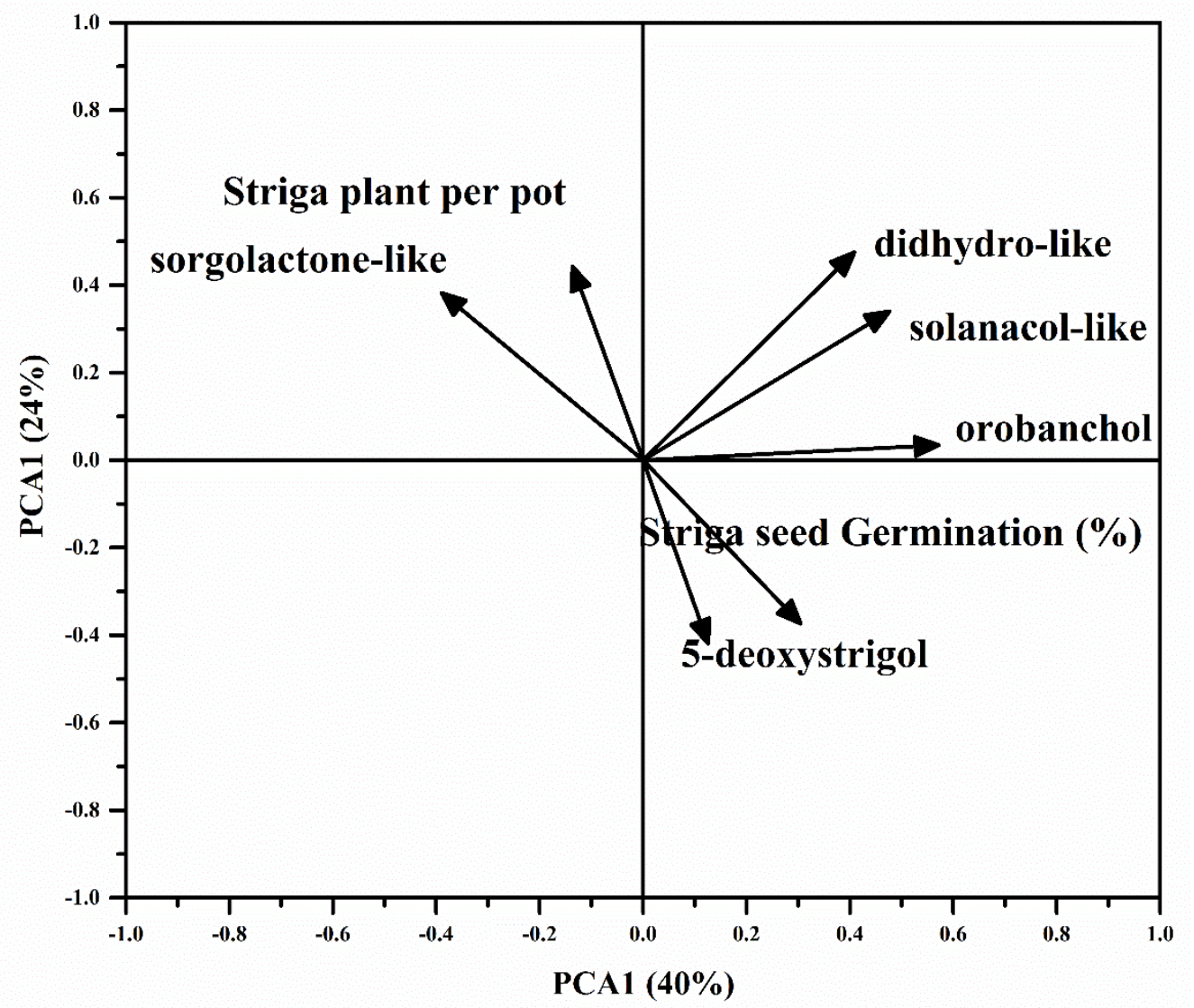

Fig. 5 Bi-plot of the first two components of a PCA based on strigolactone peak area showing relationship between strigolactone production and composition by non-host species that were intercropped with sorghum in pots, and germination of Striga seeds as induced by root exudates and the number of emergent Striga plants per pot.

Also correlation analysis showed that Striga germination as induced by the intercrops and Striga emergence on sorghum were inversely associated. Striga germination was positively correlated with the peak areas of 5-deoxystrigol, orobanchol and orobanchol, solonacollike, didehydro-orobanchol isomer like and was negatively correlated with the peak areas of sorgolactone-like (Table 2). In contrast, Striga emergence (on sorghum) was negatively correlated with the peak area of 5-deoxystrigol, didehydro-orobanchol, solonacol-like and was positively correlated with the peak areas of orobanchol and sorgolactone-like. Of the different strigolactones, 5-deoxystrigol showed the strongest significant negative 145 
correlation with Striga emergence $(\mathrm{r}=-0.82 ; P<0.04)$ (Table 2). All other strigolactones did not show significant correlations with both Striga parameters (Table 2). Regression analysis also showed that only the peak area of 5-deoxystrigol contributeds significantly to the explanation of the variation in Striga germination induced by the non-host crop species and Striga emergence in pots (Table S1; Fig S1). Interestingly, 5-deoxystrigol in the root exudates of the intercrop strongly and negatively correlated with emerged Striga plants per pot (on the intercropped sorghum) $(\mathrm{r}=-0.82 ; P<0.04)$ (Table 2) mirroring the inverse, though not significant, correlation between Striga seed germination induced by the root exudate of the intercrop and the Striga plants emerged on the intercropped sorghum $(\mathrm{r}=-0.58 ; P<0.2 ;$ Fig. 5$)$.

Table 2 also shows the correlation between different strigolactones detected in sesame and groundnut. 5-deoxystrigol and didehydro-orobanchol isomer-like were positively and significantly $(P<0.05)$ correlated. Orobanchol and sorgolactone-like showed a negative and non-significant correlation with 5-deoxystrigol, didehydro-orobanchol and solonacol-like and a weaker positive non-significant correlation with each other (Table 2). 
Table 2 Relationship between the amounts of strigolactones detected in the root exudates of sesame and groundnut and in vitro Striga germination and emergence on sorghum in a pot experiment. The amounts of various strigolactones, Striga germination and emergence were square root then transformed and related by correlation analysis.

\begin{tabular}{llllllll}
\hline & G & St & 5-d & did & orob & sorgol & sola \\
\hline G & 1 & -0.58 & 0.60 & 0.25 & 0.14 & $-0.93^{*}$ & 0.24 \\
St & -0.58 & 1 & $-0.82^{*}$ & -0.57 & 0.54 & 0.61 & -0.79 \\
5-d & 0.60 & $-0.82^{*}$ & 1 & $0.85^{*}$ & -0.53 & -0.74 & 0.57 \\
Ded & 0.25 & -0.57 & $0.85^{*}$ & 1 & -0.49 & -0.41 & 0.61 \\
Orob & 0.14 & 0.54 & -0.53 & -0.49 & 1 & 0.12 & -0.54 \\
Sogol & $-0.93^{*}$ & 0.61 & -0.74 & -0.41 & 0.12 & 1 & -0.29 \\
Solo & 0.24 & -0.79 & 0.57 & 0.61 & -0.54 & -0.29 & 1 \\
\hline
\end{tabular}

* indicates a significant correlation at the 0.05 level.

G= germination; St, Striga plants per pot; 5-d, 5-deoxystrigol; did, didehydro-orobanchol isomer-like; orob, orobanchol; sorgol, sorgolactone-like; sola, solanocol-like.

\section{Effects of Striga infection on crop growth}

Highly significant $(P<0.001)$ positive effects of intercrop on sorghum height, leaf area and total biomass were observed at harvest irrespective of the intercrop cultivar used compared to Striga infected sorghum alone (Table 3). The highest increase in sorghum height (69\%) and total biomass $(86 \%)$ occurred when sorghum was intercropped with groundnut Blue nile, and the lowest increase in height (14\%) and total biomass $(60 \%)$ with sesame Abu Sofa (Table 3). Intercropping with sesame Abu Naama resulted in the highest increase in leaf area $(83 \%)$ whereas intercropping with groundnuts Gadarif resulted in the lowest increase $68 \%$ in this trait (Table 3).

Although there was only low Striga emergence on the two millet cultivars, some minor reductions in pearl millet stem height, leaf area and total shoot biomass were observed with a reduction in stem height of 6 and $4 \%$ for cv White and Red, respectively, compared with the Striga free plants (Table 4).

Table 3 Effects of intercroping on sorghum morphological traits expressed as percentage of sole congener: 


\begin{tabular}{llll}
\hline Intercrop & \multicolumn{2}{l}{ Mean increase congener } \\
\hline & $\begin{array}{l}\text { Plant Height } \\
(\mathrm{cm})\end{array}$ & Leaf area $\left(\mathrm{cm}^{2}\right)$ & $\begin{array}{l}\text { Total shoot } \\
\text { biomass }(\mathrm{g})\end{array}$ \\
\cline { 2 - 4 } Groundnuts Gadarif & $50.21 \mathrm{a}$ & $68.76 \mathrm{~b}$ & $82.64 \mathrm{ab}$ \\
Groundnuts Blue nile & $69.13 \mathrm{a}$ & $75.57 \mathrm{ab}$ & $86.65 \mathrm{a}$ \\
Sesame Abosofa & $14.63 \mathrm{~b}$ & $82.96 \mathrm{ab}$ & $60.34 \mathrm{~b}$ \\
Sesame Abonama & $57.10 \mathrm{a}$ & $83.87 \mathrm{ab}$ & $80.53 \mathrm{ab}$ \\
\hline
\end{tabular}

-(value in intercrop Striga infected - value Striga infected sole)/value Striga infected in intercrop*100

In contrast, the stem height of Striga infected sorghum was significantly $(\mathrm{P}<0.001)$ reduced (-84 \%) compared with the Striga-free sorghum control (Table 4). Also for leaf area and total biomass, the infected pearl millet cultivars showed a lower reduction compared with Striga free plants than sorghum that showed a significant $(P<0.001)$ reduction of about $90 \%$ in leaf area and total biomass under Striga infection (Table 4). The reduction in millet height, leaf area and total biomass due to infection by Abu Naama and Gezira Striga ecotypes were similar to that caused by the Gadarif ecotype (data not shown).

Table 4 Effects of infection by Striga Gadarif on pearl millet and sorghum change in Striga infected plants (\%)

\begin{tabular}{lllll}
\hline Cultivars & Plant height $\mathrm{cm}$ & Leaf area $\left(\mathrm{cm}^{2}\right)$ & $\begin{array}{c}\text { Total shoot } \\
\text { biomass }(\mathrm{g})\end{array}$ & $\begin{array}{l}\text { Head weight } \\
(\mathrm{g})\end{array}$ \\
\hline Red millet & $-3.81 \mathrm{~b}$ & $-33.37 \mathrm{~b}$ & $-28.45 \mathrm{~b}$ & -4.66 \\
White millet & $-5.77 \mathrm{~b}$ & $-25.68 \mathrm{~b}$ & $-23.49 \mathrm{~b}$ & -24.8 \\
sorghum & $-84.52 \mathrm{a}$ & $-91.80 \mathrm{a}$ & $-88.41 \mathrm{a}$ & -100.00 \\
\hline
\end{tabular}




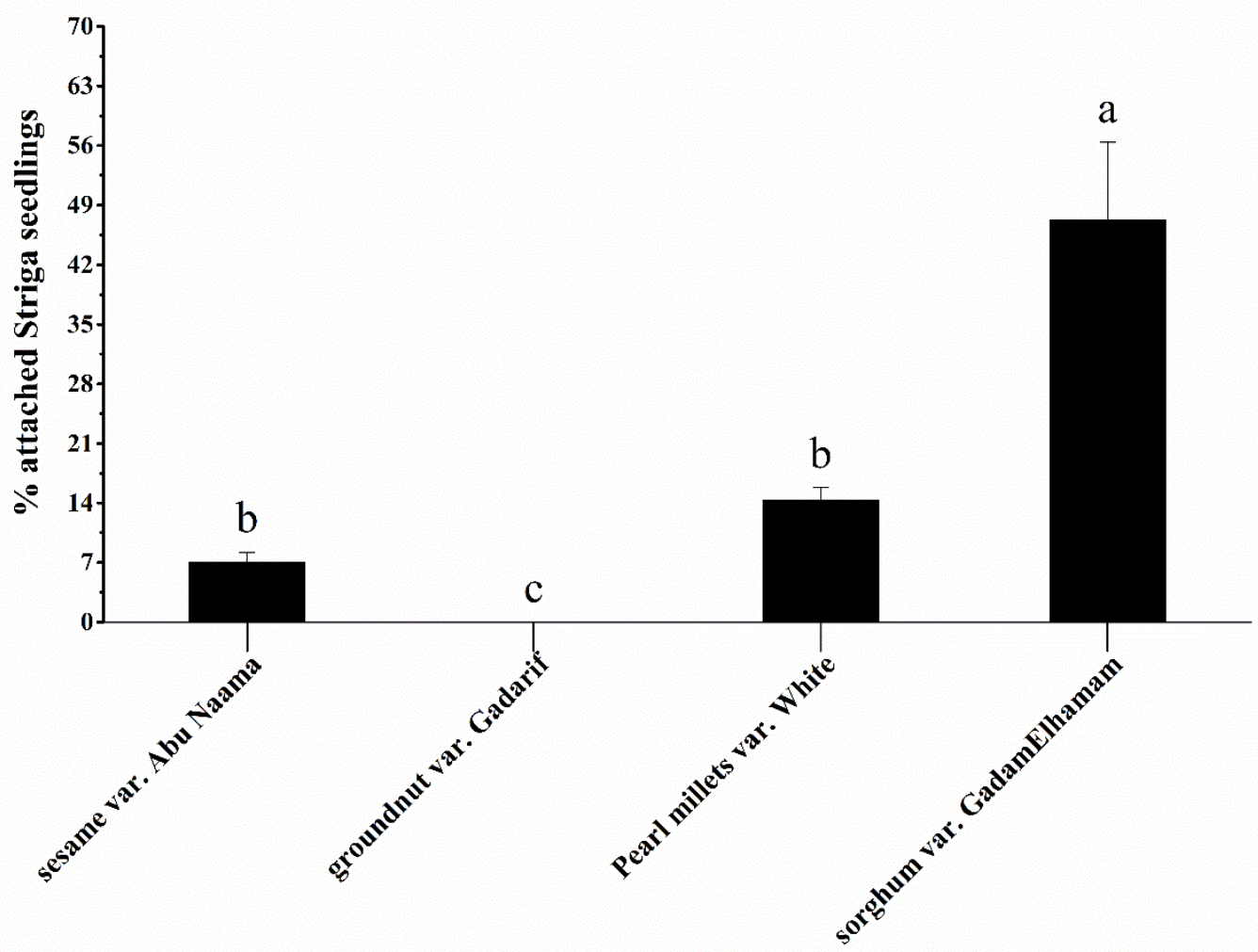

Fig. 6 Differences in Striga attachment to the roots of millet, sesame, groundnut and sorghum as revealed by rhizotrons. Bars represents mean percentage attached Striga seedlings \pm standard error $(\mathrm{n}=4)$. Least significant differences of means at $\mathrm{P}=0.05$ by ANOVA test. Letters indicate the different significance groups after Duncans' pairwise comparisons $(P<0.05)$.

In the rhizotron, 10 days post inoculation only very few Striga seedlings were attached and initiated penetration into the roots of sesame and millet compared to the roots of sorghum, while on groundnut at up to 28 days post inoculation, no Striga seedlings had established attachment or penetration. Roots of sesame and millet supported only $7 \%$ and $14 \%$ of the inoculated Striga seeds, respectively, compared to sorghum roots which supported $47 \%$ of the inoculated Striga seeds (Fig 6). Attached seedlings on roots of sesame and millet observed in the 28 days of co-cultivation were characterized by shorter shoots with thicker malformed leaves, delayed and clearly arrested development, with no formation of secondary haustoria as well as higher mortality of the parasite compared to the ones 
attached to sorghum roots, which developed normally and formed more than six leaf-pairs (Fig 7a-c). Although some of the attached seedlings on millet roots formed up to six leaf pairs, most seedlings appeared weak, ill-nourished, showed etiolated shoots and failed to develop further compared to those attached to sorghum roots which appeared wellnourished, showed no etiolated shoots and developed normally (Fig.7g-1).
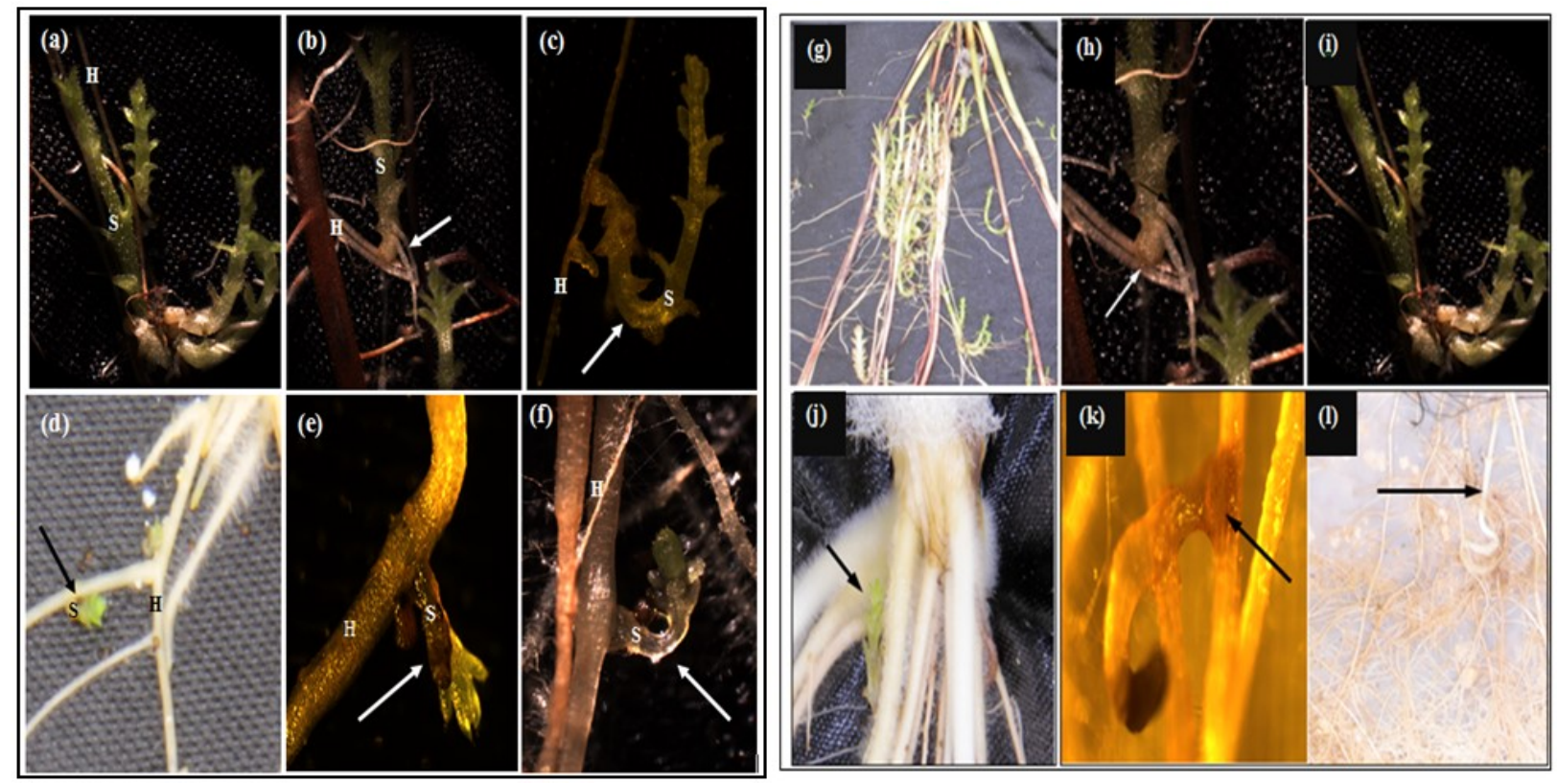

Fig. 7: Sesame incompatibility responses to infection of Striga hermonthica, above Striga seedlings infecting sorghum (a-c) attachments frequency in (a), white arrow indicating secondary haustoria in (b), normal seedling growth in (c); below: Striga seedlings infecting sesame roots (d-f) low attachment frequency and arrested growth in (d), Resistance phenotypes expressed by pearl millet upon Striga infection. Above Striga seedlings infecting sorghum (g-i) attachments frequency in (g), white arrow indicating normal attachment point in (h) and normal seedling growth in (i); Striga seedlings infecting millets (j-l) low attachments frequency and arrested growth in (j), black arrow indicating hypersensitive response at attachment point in (k) and etiolated abnormal Striga seedling in (1), H indicates host root, S Striga seedling. White arrow indicating short shoot with the seed coat still attached and arrested leaf expansion (e) and abnormal leaves is shown in (f), $\mathrm{H}=$ sesame roots; $\mathrm{S}=$ Striga seedlings. 


\section{Discussion}

Promotion of growth and yield of sorghum on Striga-infested fields by intercropping or rotation with non-host (leguminous) crops have been well documented (Carson 1989; Carsky et al., 1994; Ransom, 1999; Tenebe et al., 2002; Khan et al., 2007b). These nonhost intercrops reduce infection of sorghum by Striga through a number of possible different mechanisms of which depletion of the Striga soil seed bank by suicidal germination seems to be the most important (Sauerborn et al., 1999; Oswald \& Ransom, 2001; Babiker, 2002; Khan et al., 2002; Schulz et al., 2003). Benson, (1982) showed that the relative effectiveness of Striga-non-host intercrop genotypes of soybean and cowpea to induce Striga germination in the laboratory was highly correlated with their effectiveness in reducing parasitism on susceptible sorghum in the field. In the rhizotrons, compared with sorghum, Striga showed fewer attachments on sesame and millets or no attachment on groundnut and much slower development and a large portion of germinated seeds failed to attach or died later (Fig. 7a-1). This indicates low penetration efficiency and physiological or biochemical incompatibility reactions resulting in a difficulty of obtaining nutrients andlor metabolites essential for sustaining normal growth of the parasite. A similar phenomenon was reported for sorghum cultivar Framida, a Striga resistant sorghum variety (Arnand et al., 1999), maize line ZD05 (Amusan et al., 2008) and in rice varieties including Nipponbare, Koshihikari, CG14, NERICA1 and NERICA10 (Gurney, et al 2006; Samejima et al., 2016a) and was attributed to blockage of penetration and translocation of assimilates and/or other host metabolites essential for growth and development of the parasite, thus leading to haustorial collapse and/or inhibition of the haustorial development culminating in slow growth and/or death of the parasite.

In the present study we showed that the intercrops sesame and groundnut significantly delayed time and number of Striga emergence on intercropped sorghum compared with sorghum alone (Fig. 4A). Delaying Striga emergence has been demonstrated to result in a reduction of its deleterious effects on sorghum (Gurney et al., 1999; Van Ast et al., 2000; Mohemed et al., 2016). The combination of this delayed infection/emergence with the lower total Striga density in the intercropped treatments resulted in a significant increase 
in sorghum stem height, leaf area and total biomass compared with the Striga-infected sorghum grown without an intercrop (Fig. 4; Table 3).

Varieties of sesame and groundnut were frequently mentioned as effective Striga trap crops and/or intercrops. In the Sudan, Andrews (1945), based on pot experiments, reported that sowing groundnut, cowpea, and dolichos bean in a Striga- infested soil considerably reduces the parasite seed bank. Also Babiker et al., (1996; 2002) showed that when sorghum was intercropped with groundnut, cowpea and dolichos beans Striga emergence was generally lower than on sorghum alone. Moreover, the Striga plants that did emerge had less seeds per capsule and sorghum grain yield increased by several fold compared to the corresponding sorghum treatment without intercrop. The reduction in Striga infection by intercrops may probably be explained by a combination of mechanisms ranging from induction of suicidal germination, increasing available nitrogen, allelopathic effects, shading, smothering effects, reduction of soil temperature and/or increase of humidity which results in reduction of the parasite transpiration (Parker and Riches, 1993; Berner et al., 1996c; Press and Gurney, 2000b Oswald et al., 2002a). Further, semiochemicals in the intercrop roots exudates may perturb temporal and/or spatial growth of the parasite radicle thus reducing attachments to the host roots and consequently parasitism (Khan et al., 2002). Finally, the differences in root exudates between sorghum and the intercrops may also have consequences for the microbial community in the sorghum rhizosphere and through this way provide a protective effect against Striga infection. Even differences in the root exudate of sorghum genotypes grown alone were recently shown to result in differences in composition of rhizosphere microbial communities (Schlemper et al., 2017).

In this study we showed that the root exudates of sesame, groundnut and the synthetic strigolactone GR24 induced significant and comparable germination in Striga (Fig. 3). This is in line with results of Bebawi (1991) who bio-assayed sesame root exudates among other crops including sunflower, guar and cotton for Striga seed germination and concluded that these crops may significantly contribute to the depletion of the Striga seedbank through suicidal germination. Likewise, De Groote et al., (2010) showed that soybean provoked suicidal germination of Striga and reduced the Striga seed bank in the soil when 
intercropped with maize. In our study we complemented the Striga seed germination assays with the analysis of strigolactone germination stimulants present in the exudates. We showed that root exudates of sesame and groundnut cultivars contain high amounts of 5deoxystrigol (Fig. 3A-D). In our lab (Mohemed et al., submitted) synthetic showed that 5deoxystrigol at $0.02 u \mathrm{M}$ elicited $50 \%$ and $60 \%$ seed germination in the Striga Abu Naama ecotype. Our statistical analysis revealed that the amount of 5-deoxystrigol in the root exudates of sesame and groundnut showed a positive, though non-significant, correlation with in vitro Striga germination and a strong, significant, negative correlation with Striga emergence on subsequent sorghum suggesting occurrence of suicidal germination (Table 2). Also regression analysis (Table S1; Fig S1) and PCA (Fig. 5) showed that 5deoxystrigol is the best predictor for Striga germination and emergence providing indirect prove for occurrence of suicidal germination. This suggests that intercropping with cultivars which produce high amounts of 5-deoxystrigol can result in better Striga suicidal germination. While intercropping may offer a simple and environmentally friendly Striga control option, crop substitution (e.g. cultivation of pearl millet instead of sorghum or sorghum instead of pearl millet) under heavy infestation of either crop may help to avoid complete yield loss in a major crop and provide subsistence farmers with alternative cereal staple food. Such practice was recommended in some parts of the African content and was reported to be very useful in Striga-endemic areas (Obilana et al., 1992). In this study we investigated the response of two pearl millet cultivars to the sorghum Striga ecotypes (Gadarif, Gezira and Abu Naama). In the pot experiments we showed that both pearl millet cultivars possess a good level of resistance against the tested Striga ecotypes (Fig. 4C; Table 4). The finding that Striga ecotypes Gadarif, Abu Naama and Gezira are more virulent on sorghum than on pearl millet confirms the presence of host specificity. Existence of two crop specific $S$. hermonthica ecotypes has been reported in Sudan before (Wilson Jones, 1955). One is virulent on sorghum and occurs in the heavy clay soils (where sorghum is mostly grown), the other is virulent on pearl millet and occurs mainly in the sandy soils of Kordofan (where pearl millet is the dominant cereal). It has been reported that resistance to Striga can occur pre- and/or post germination (Haussmann et al., 2000; 
Mohamed et al., 2003; Yoder, et al., 2010; Timko, et al., 2013). Post-germination resistances encompass interruption of host root penetration by the parasite and/or inhibition of subsequent development. In the rhizotron experiments using pre-germinated Striga seeds we showed that few attachements were realized on pearl millet roots. Furthermore, the attached seedlings displayed slow and abnormal growth. They were bleached, etiolated and/or failed to initiate more than 4 pairs of leaves (Fig $7 \mathrm{~g}-\mathrm{k}$ ). In contrast on sorghum, most Striga seedlings developed fast, and initiated more than 4 pairs of green non-etiolated normally growing leaves (Fig $7 \mathrm{~g}$-i). Reduced growth of Striga on host roots has been attributed to a failure of the parasite to attach and make proper connections to the host's xylem tissue (Arnaud et al., 1999; Samejima et al., 2016a). This indicates incompatibility and low penetration efficiency. The expressed post-germination resistance in pearl millet could be due to failure to supply sufficient haustorial initiation factors and/or nutrients to the parasite. The results also suggest that the tested Striga ecotypes obviously are more specific to sorghum than millet due to post-germination differences. However, Parker and Reid (1979) in West Africa demonstrated that Striga host specificity (pearl millet resistances) could also be due to differences in germination inducing activity by the root exudates. Parker and Reid (1979) reported failure of pearl millet root exudates to stimulate germination of the Striga ecotype from sorghum. In our study, indeed the root exudates of both pearl millet cultivars elicited low in vitro germination compared to that elicited by root exudates of the sorghum cultivar (Fig. 3). Production of less active germination stimulant is a well-documented resistance mechanism against Striga infection (Hess, et al., 1992; Ejeta, et al., 2007b; Mohemed, et al., 2016; Gobena, et al., 2017). Our LC/MS/MS analyses showed that the root exudates of both pearl millet cultivars typically contain low amounts of 5-deoxystrigol, sorgomol and orobanchol and large amounts of orobanchyl acetate (Fig. 3F-G). Orobanchyl acetate has been earlier characterized in soybean and cowpea and in tomato and claimed to be formed by the acetylation of orobanchol (Rani, et al., 2008). Orobanchyl acetate, an orobanchol-like strigolactone, has been reported to be less active in inducing germination of Striga seeds collected from sorghum than their strigol-type congeners (Nomura, 2013). In general, acetylated strigolactones are 10- to - 
100-fold less active than their hydroxy-congeners (Yoneyama et al., 2009). Therefore the notable resistance of pearl millet to the Striga sorghum ecotype may be attributed, at least, in part to the presence of high amounts of orobachyl actetate in pearl millet root exudates at the expense of the more active strigolactones viz 5-deoxystrigol, sorgomol or strigol. It is intriguing that just as in sorghum low germination based resistance is due to a shift from strigol-type to orobanchol-type strigolactones (Gobena et al., 2017). However, in contrast to sorghum, in millet not only the type is switched, but there is also acetylation occurring. It would be interesting to see if Striga ecotypes that parasitise pearl millet are more sensitive to the orobanchol-type strigolactone orobanchyl acetate. Resistance to Striga in pearl millet was documented for few semi-wild lines and some genepool accessions, however the underlying mechanisms have not been examined so far (Kountche et al., 2013). This study suggests involvement of both pre- and post-attachment resistance in pearl millet due to differences in the amount and/or composition of strigolacones in the pearl millet root exudate and the inability of Striga seedlings to develop on pearl millet roots, respectively.

\section{Conclusions}

The reduction in parasite infection on sorghum intercropped with sesame or groundnut is largely due to secretion by the intercrops of specific active germination stimulants into the rhizosphere that induced germination of the parasite. Suicidal germination has been suggested by many authors to be one of the mechanisms by which intercrops can reduce Striga infection in the main crop. The strong correlations between in vitro strigolactones, germination inducing activity and reduction of Striga infection on the host sorghum reported in this study suggests that suicidal germination is indeed the major cause for the intercrop effect. This knowledge should help in the selection/breeding or engineering of intercrops cultivars that provide better control of Striga. In addition, the production of high amounts of less active germination stimulants viz., orobanchyl acetate and low amounts of active stimulants viz., 5-deoxystrigol conferred millet pre-attachment resistance against Striga sorghum ecotypes. However, post-attachment resistance in the pearl millet cultivars 
makes an important contribution to their suitability as substitution crop. Knowledge of the strigolactone profiles of a potential substitution crop as well as the crop to be substituted and insight in the strigolactone sensitivity of the prevalent Striga ecotype will be crucial to implementation of this strategy. 


\section{References}

Abunyewa, A. A., and Padi, F. K. (2003). Changes in soil fertility and Striga hermonthica prevalence associated with legume and cereal cultivation in the Sudan savannah zone of Ghana. Land Degradation \& Development, 14(3), 335-343.

Rasha, A.M.A., El-Hussein, A. A., Mohamed, K.I., and Babiker, A.G.T., (2009). Specificity and Genetic Relatedness among Striga hermonthica Strains in Sudan. Life Science, 3: 1159-1166

Akiyama, K., Ogasawara, S., Ito, S. and Hayashi, H. (2010). Structural requirements of strigolactones for hyphal branching in AM fungi. Plant Cell Physiology 51: 1104-1117.

Alder, A., Jamil, M., Marzorati, M., Bruno, M., Vermathen, M., Bigler, P., ... and Al-Babili, S. (2012). The path from $\beta$-carotene to carlactone, a strigolactone-like plant hormone. Science, 335(6074), 1348-1351.

Amusan IO, Rich PJ, Menkir A, Housley T, Ejeta G (2008). Resistance to Striga hermonthica in a maize inbred line derived from Zea diploperennis. New Phytologist, 178(1), 157-166.

Andrews, F. W. (1945). The parasitism of Striga hermonthica Benth. on Sorghum spp. under irrigation: I. Preliminary results and the effect of heavy and light irrigation on Striga attack. Annals of Applied Biology, 32(3), 193-200.

Ariga, E. S. (1996). Isolation and bioassay of Striga hermonthica seed germination stimulants from non-host crops and field testing for control efficacy (Doctoral dissertation, Ph. D. dissertation, Department of Crop Science, University of Nairobi, Kenya) Chapter1 pp32-61.

Arnaud, M. C., Véronési, C., \& Thalouarn, P. (1999). Physiology and histology of resistance to Striga hermonthica in Sorghum bicolor var. Framida. Functional Plant Biology, 26 (1), 63-70.

Atera, E.A., Itoh, K., and Onyango, J.C., (2011). Evaluation of ecologies and severity of Striga weed on rice in sub-Saharan Africa. Agriculture and biology journal of North America, 2(5), 752-760.

Awad, A. A., Sato, D., Kusumoto, D., Kamioka, H., Takeuchi, Y. and Yoneyama, K.. (2006). Characterization of strigolactones, germination stimulants for the root parasitic plants Striga and Orobanche, produced by maize, millet and sorghum. Plant Growth Regulation, 48: 221-227.

Babalola O. O, Osir E. O, Sanni A. I., (2002). Characterization of potential ethylene-producing rhizosphere bacteria of Striga-infested maize and sorghum. African Journal of Biotechnology 1:67-69.

Babiker, A. G. T. (2007). Striga: The spreading scourge in Africa. Regulation of Plant Growth and Development. 43.1: 74-87.

Babiker, A. G.T. Butler, L. and Ejeta, G., (1996). Integrated use of Striga resistant sorghum varieties with cultural and chemical control. In: The International Conference on Genetic Improvement of sorghum and Pearl Millet. Taxas A and M University Research and Extension Centre, Luubbock, USA, pp 517-524.

Babiker, AGT (2002). Striga control in Sudan: An integrated approach. In, Leslie, J F(ed). Sorghum and Millet Diseases, pp 159-163. Iowa State Press.

Bebawi, F. F., \& Eplee, R. E., (1986). Efficacy of ethylene as a germination stimulant of Striga hermonthica seed. Weed Science, 34 (5), 694-698.

Bebawi, F., and Michael, A., (1991). Bioassay of some economic crops of the Sudan to Striga germination and parasitization, In: 5. International Symposium of Parasitic Weeds, Nairobi (Kenya), 24-30 Jun 1991: CIMMYT).

Bebawi, F.F., R.E. Eplee, C.E. Harris, and R.S. Norris., (1984). Longevity of witchweed (Striga asiatica) seed. Weed Science. 32:494-507.

Benson, J. M. (1982). Weeds in Tropical Crops: Review of Abstracts on Constraints in Production Caused by Weeds in Maize, Rice, Sorghum-millet, Groundnuts, and Cassava. Food \& Agriculture Organization. (Vol. 32) 1952-1980. 
Berner, D., Carsky, R., Dashiell, K., Kling, J., and Manyong, V., (1996). A land management based approach to integrated Striga hermonthica control in sub-Saharan Africa. Outlook on Agriculture 25(3), 157-164.

Bouwmeester, H.J., Roux, C., Lopez-Raez, J.A., and Becard, G., (2007). Rhizosphere communication of plants, parasitic plants and AM fungi. Trends in plant science 12, (5), 224-230.

Cardoso, C., Charnikhova, T., Jamil, M., Delaux, P. M., Verstappen, F., Amini, M., and Bouwmeester, H. (2014). Differential activity of Striga hermonthica seed germination stimulants and Gigaspora rosea hyphal branching factors in rice and their contribution to underground communication. PloS one, 9(8), e104201.

Carsky RJ, Berner DK, Oyewole BD, Dashiell K, Schulz S., (2000). Reduction of Striga hermonthica parasitism on maize using soybean rotation. International Journal of Pest Management 46:115-120.

Carsky, R. J., Singh, L., \& Ndikawa, R. (1994). Suppression of Striga hermonthica on sorghum using a cowpea intercrop. Experimental Agriculture, 30(3), 349-358.

Carson, A. G. (1989). Effect of intercropping sorghum and groundnuts on density of Striga hermonthica in the Gambia. International Journal of Pest Management, 35(2), 130-132.

Charnikhova, T. V., Gaus, K., Lumbroso, A., Sanders, M., Vincken, J. P., De Mesmaeker, A., \& Bouwmeester, H. J. (2017). Zealactones. Novel natural strigolactones from maize. Phytochemistry, 137, 123-131.

Cheng, X., Floková, K., Bouwmeester, H., \& Ruyter-Spira, C. (2017). The role of endogenous strigolactones and their interaction with $\mathrm{ABA}$ during the infection process of the parasitic weed Phelipanche ramosa in tomato plants. Frontiers in plant science, 8, p. 392.

Cook, C.E., Whichard, L.P., Turner, B., Wall, M.E., and Egley, G.H. (1966). Germination of witchweed (Striga lutea Lour.): Isolation and properties of a potent stimulant. Science 154: 1189-1190.

De Groote, H., Rutto, E., Odhiambo, G., Kanampiu, F., Khan, Z., Coe, R., \& Vanlauwe, B. (2010). Participatory evaluation of integrated pest and soil fertility management options using ordered categorical data analysis. Agricultural Systems, 103 (5), 233-244.

Dawoud, D. A., \& Sauerborn, J., (2015). Efficiency of Some Crops in Inducing Suicidal Germination of the Parasitic Weed, Striga hernonthica Del Benth. Egyptian Journal of Biological Pest Control 25, (3), 735 738.

Drennan, D. S. H., and S. O. El-Hiweris. (1979). Changes in growth regulating substances in Sorghum vulgare infected by Striga hermonthica. Pages 144-155 in L. J. Musselman, A. D. Worsham, and R. E. Eplee, eds. Second International Symposium on Parasitic Weeds. North Carolina State University, Raleigh, $\mathrm{NC}$, USA.

Ejeta, G. and Gressel, J., eds. (2007a). Integrating New Technologies for Striga Control: Towards Ending the Witch-Hunt. World Scientific Publishing Co. Pte. Ltd., Singapore. pp 9-10.

Ejeta, G., Rich, P.J., and Mohamed, A., (2007b). Dissecting a complex trait to simpler components for effective breeding of sorghum with a high level of Striga resistance. Integrating new technologies for Striga control. Towards ending the witch-hunt. World Scientific Publishing Co. Pte. Ltd, Singapore, 87-98.

Emechebe A.M., Ahonsi M.O., Kureh I., Alabi S.O., Gbéhounou G. and Adango E. (2003). Ability of excised root and stem pieces of maize, cowpea and soybean to cause germination of Striga hermonthica seeds. Crop Protection. 22, 347-353.

Emechebe, A., Ellis-Jones, J., Schulz, S., Chikoye, D., Douthwaite, B., Kureh, I., et al., (2004). Farmers' perception of the Striga problem and its control in northern Nigeria. Experimental Agriculture. 40(2), 215-232.

Eplee RE., 1981. Striga's status as a plant parasite in the United States. Plant Disease. 65:951-954.

Eplee RE., 1992.Witchweed (Striga asiatica): an overview of management strategies in the USA. Crop Protection 11:3-7. 
Gobena, D., Shimels, M., Rich, P. J., Ruyter-Spira, C., Bouwmeester, H., Kanuganti, S. Mengiste, T. and Ejeta, G., (2017). Mutation in sorghum LOW GERMINATION STIMULANT1 alters strigolactones and causes Striga resistance. PNAS, 114, 4471-4476.

Goldwasser, Y., and Rodenburg, J., (2013). Integrated agronomic management of parasitic weed seed banks, in Parasitic Orobanchaceae, Springer, 393-413.

Gurney, A. L., Press, M. C., \& Scholes, J. D. (1999). Infection time and density influence the response of sorghum to the parasitic angiosperm Striga hermonthica. The New Phytologist, 143 (3), 573-580.

Gurney AL, Slate J, Press MC, Scholes JD. (2006). A novel form of resistance in rice to the angiosperm parasite Striga hermonthica. New Phytologist 169: 199-208.

Hassan, M. M., Gani, M. E. A., \& Abdel El Gabar, E. B., (2013). Control of Striga hermonthica on sorghum inoculated with Fenugreek (Trigonella Foenum-Graecum) seeds powder. Journal of Current Research in Science 1, (6), 583-587.

Haussmann, B. I. G., Hess, D. E., Welz. H. G and Geiger, H. H. (2000). Improved Methodologies for Breeding Striga-resistant sorghums. Field Crops Research 66, 195-201.

Hess, Dale E., Gebisa Ejeta, and Larry G. Butler., (1992). Selecting sorghum genotypes expressing a quantitative biosynthetic trait that confers resistance to Striga. Phytochemistry 31.2: 493-497.

Joel, D.M., Hershenhorn, Y., Eizenberg, H., Aly, R., Ejeta, G., Rich, P. J., Ranson, J.K., Sauerborn, J., Rubiales. D., (2006). Biology and management of weedy root parasites. Horticultural reviews, 33:267-349.

Kabambe, V., Katunga, L., Kapewa, T., and Ngwira, A., (2008). Screening legumes for integrated management of witchweeds (Alectra vogelii and Striga asiatica) in Malawi. African Journal of Agricultural Research 3(10), 708-715.

Kgosi RL, Zwanenburg B, Mwakaboko AS, Murdoch AJ., (2012). Strigolactone analogues induce suicidal germination of Striga spp. in soil. Weed Research 52:197-203.

Khan, Z. R., Midega, C. A., Hassanali, A., Pickett, J. A., \& Wadhams, L. J. (2007). Assessment of different legumes for the control of Striga hermonthica in maize and sorghum. Crop Science, 47(2), 730-734.

Khan, Z.R., Hassanali, A., Overholt, W., Khamis, T.M., Hooper, A.M., Pickett, J.A., et al., (2002). Control of witchweed Striga hermonthica by intercropping with Desmodium spp., and the mechanism defined as allelopathic. Journal of chemical ecology 28(9), 1871-1885.

Kountche, B.A., Hash, C.T., Dodo, H., Laoualy, O., Sanogo, M.D., Timbeli, A., Vigouroux, Y., This, D., Nijkamp, R. and Haussmann, B.I., (2013). Development of a pearl millet Striga-resistant genepool: Response to five cycles of recurrent selection under Striga-infested field conditions in West Africa. Field crops research, 154, pp.82-90.

Kureh, I., Kamara, A., and Tarfa, B., (2006). Influence of cereal-legume rotation on Striga control and maize grain yield in farmers' fields in the Northern Guinea savanna of Nigeria. Journal of Agriculture and Rural Development in the Tropics and Subtropics (JARTS) 107(1), 41-54.

Labrada, R. (2008). Farmer training on parasitic weed management, In: Progress on Farmer Training in Parasitic Weed Management (Labrada, R., ed.), pp. 1-5. Rome: FAO.

Lane, J.A., Bailey, J.A., and Terry, P.J., (1991). An in vitro growth system for studying the parasitism of cowpea (Vigna unguiculata (L.) Walp.) by (Striga gesnerioides (Willd.) Vatke). Weed Research. 31: 211-217.

Lendzemo, Venasius W., Thomas W. Kuyper, Radoslava Matusova, Harro J. Bouwmeester, and A. van Ast (2007). Colonization by arbuscular mycorrhizal fungi of sorghum leads to reduced germination and subsequent attachment and emergence of Striga hermonthica. Plant signaling \& behavior 2, no. 1: 5862.

Lopez-Raez, J. A., Charnikhova, T., Gómez-Roldán, V., Matusova, R., Kohlen, W., De Vos, R., ... and Bouwmeester, H. (2008). Tomato strigolactones are derived from carotenoids and their biosynthesis is promoted by phosphate starvation. New Phytologist, 178(4), 863-874. 
Mangnus, E.M., and Zwanenburg, B. (1991). Design and synthesis of germination stimulants for seeds of Striga and Orobanche spp. In: Progress in Orobanche Research: Proceedings of the International Workshop on Orobanche Research, Obermarchtal (D), 19-22 August 1989, Wegmann, K., and Musselman, L.J., eds (Tübingen, Germany: Eberhard-Karls-Universität), pp. 157-166.

Matusova, R., van Mourik, T., and Bouwmeester, H.J., (2004). Changes in the sensitivity of parasitic weed seeds to germination stimulants. Seed Science Research, 14(04), 335-344.

Mohamed, A., Ellicott, A., Housley, TL., and Ejeta, G., (2003). Hypersensitive Response to Striga Infection in Sorghum. Crop Science 43(4): 1320-1324.

Mohamed, K.I., Bolin, J.F., Musselman, L.J., and Peterson, A., (2007). Genetic diversity of Striga and implications for control and modeling future distributions. In: Integrating New Technologies for Striga Control-Towards Ending the Witch-hunt, World Scientific, Singapore, 71-84.

Mohemed, N., Charnikhova, T., Bakker, E. J., Ast, A., Babiker, A. G., \& Bouwmeester, H. J. (2016). Evaluation of field resistance to Striga hermonthica (Del.) Benth. in Sorghum bicolor (L.) Moench. The relationship with strigolactones. Pest Management Science, 72 (11), 2082-2090.

Mohemed, N., Charnikhova, T., Fradin, F. E., Rienstra, J., Babiker, A.G., and Bouwmeester, H. (2017). Genetic variation in strigolactones and its effect on resistance and susceptibility to Striga hermonthica in Sorghum bicolor. Manuscript submitted for publication.

Motonami, N., Ueno, K., Nakashima, H., Nomura, S., Mizutani, M., Takikawa, H., \& Sugimoto, Y. (2013). The bioconversion of 5-deoxystrigol to sorgomol by the sorghum, Sorghum bicolor (L.) Moench. Phytochemistry, 93, 41-48.

Murdoch, A.J. \& Kunjo, E.M. (2003). Depletion of natural soil seedbanks of Striga hermonthica in West Africa under different integrated management regimes. Aspects of Applied Biology 69, 261-268.

N'Cho SA. (2014). Socio-economic impacts and determinants of parasitic weed infestation in rainfed rice systems of sub-Saharan Africa. PhD thesis, Wageningen University Wageningen, the Netherlands.

Nomura S, Nakashima H, Mizutani M, Takikawa H, Sugimoto Y., (2013). Structural requirements of strigolactones for germination induction and inhibition of Striga gesnerioides (Willd.) Vatke. seeds. Plant cell reports 32: 829-838.

Obilana, A.B., Ramaiah, K.V., (1992). Striga (witchweeds) in sorghum and millet: knowledge and future research needs. In: de Milliano, W.A.J., et al. (Eds.), Sorghum and Millet Diseases: A Second World Review. International Crops Research Institute for the Semi-Arid Tropics (ICRISAT), Patancheru, AP 502 324, pp. 187-201.

Oswald, A., and Ransom, J., (2001). Striga control and improved farm productivity using crop rotation. Crop Protection 20(2), 113-120.

Oswald, A., Ransom, J.K., Kroschel, J. and Sauerborn, J. (2002). Intercropping controls Striga in maize based farming systems. Crop Protection 21, 367-374.

Parker, C., and Reid, D.C., (1979). Host specificity in Striga species-some preliminary observations. In: Proceedings, Second International Symposium on Parasitic Weeds Pages 79-90, 16-19 July 1979, North Carolina, USA. Available from Crop Science Department, North Carolina State University, Raleigh, NC 27650, USA.

Parker, C., Riches, C.R., (1993). Parasitic Weeds of the World: Biology and Control. CAB International, Wallingford, UK, $332 \mathrm{pp}$.

Press, M.C., Gurney, A.L., (2000). Plant eats plant: sap-feeding witchweeds and other parasitic angiosperms. Biologist 47, 189-193.

Rani, K., Zwanenburg, B., Sugimoto, Y., Yoneyama, K., and Bouwmeester, H. J. (2008). Biosynthetic considerations could assist the structure elucidation of host plant produced rhizosphere signalling 
compounds (strigolactones) for arbuscular mycorrhizal fungi and parasitic plants. Plant Physiology and Biochemistry, 46(7), 617-626.

Ransom, J.K. (1999). The status qouo of Striga control: Cultural, chemical and integrated aspects. In: Advances in Parasitic Weed Control at On-farm level Vol.1, Joint Action to Control Striga in Africa. Kroschel, J, Mercer Quarshie, H and Sauerborn, J (eds), pp.133-143. Weikersheim: Margraf.

Ransom JK, Njorge J. 1991. Seasonal variation in ethyleneinduced germination of Striga hermonthica in western Kenya. In: Ransom JK, Musselman LJ, Worsham AD, Parker C, eds. Proceedings of the fifth international symposium on parasitic weeds, Nairobi, Kenya. CIMMYT, 137- 43.

Rezig, A. A. M., Abdelhalim, T. S., Hassan, M. M., Abusin, R. M. A., Eltayeb, H. A., Samejima, H., \& Babiker, A. G. T., (2016). Influence of cowpea root powder and exudates on germination and radicle length in Striga hermonthica, sorghum and pearl millet strains. African Journal of Agricultural Research, 11(24), 2082-2091.

Rodenburg J, Bastiaans L, Weltzien E, Hess DE., (2005). How can field selection for Striga resistance and tolerance in sorghum be improved? Field Crops Research, 93:34-50

Rubiales D, Verkleij J, Vurro M, Murdoch AJ, Joel DM (2009). Parasitic plant management in sustainable agriculture, Weed Research 49:1-5

Sato, D., Awad, A.A., Chae, S.H., Yokota, T., Sugimoto, Y., Takeuchi, Y. and Yoneyama, K., (2003). Analysis of strigolactones, germination stimulants for Striga and Orobanche, by high-performance liquid chromatography/tandem mass spectrometry. Journal of Agricultural and Food Chemistry, 51(5), pp.1162-1168.

Samejima H, Babiker AG, Mustafa A, Sugimoto Y. (2016a). Identification of Striga hermonthica-resistant upland rice varieties in Sudan and their resistance phenotypes. Frontiers in Plant Science 7: 634.

Samejima, H., Babiker, A. G., Takikawa, H., Sasaki, M., \& Sugimoto, Y. (2016b). Practicality of the suicidal germination approach for controlling Striga hermonthica. Pest management science, 72(11), 2035-2042.

Sunderland, N. (1960). The production of the Striga and Orobanche germination stimulants by maize roots: I. The number and variety of stimulants. Journal of Experimental Botany, 11(2), 236-245.

Sauerborn, J., Sprich, H. and Mercer-Quarshie, H. (1999). Parasitic weed infestation of maize and sorghum as influenced by crop rotation. In Advances in Parasitic Weed Control at On-farm Level, Vol I, Joint Action to Control Striga in Africa, 161-169 (Eds J. Kroschel, H. Mercer-Quarshie and J. Sauerborn). Weikersheim, Germany: Margraf Verlag.

Schlemper, T. R., Leite, M. F., Lucheta, A. R., Shimels, M., Bouwmeester, H. J., van Veen, J. A., \& Kuramae, E. E. (2017). Rhizobacterial community structure differences among sorghum cultivars in different growth stages and soils. FEMS Microbiology Ecology, 93(8).

Scholes, J. D. and Press, M. C. (2008). Striga infestation of cereal crops - an unsolved problem in resource limited agriculture. Current Opinion in Plant Biology, 11(2): 180-186.

Schulz, S., Hussaini, M. A., Kling, J. G., Berner, D. K., \& Ikie, F. O. (2003). Evaluation of integrated Striga hermonthica control technologies under farmer management. Experimental Agriculture, 39(1), 99-108.

Siame BK, Wood K, Ejeta G, Weerasuriya Y. \& Butler LG. (1993). Isolation of strigol, a germination stimulant for Striga asiatica from host plants. Journal of Agricultural Food Chemistry 41, 1486-1491.

Spallek, T., Mutuku, M., and Shirasu, K., (2013). The genus Striga: a witch profile. Molecular plant pathology, 14(9), 861-869.

Sun Z, Matusova R, Bouwmeester HJ., (2007). Germination of Striga and chemical signalling involved: a target for control methods. In: Gressel J, Ejeta G (eds). Integrating new technologies for Striga control: towards ending the witch-hunt. World Scientific, Singapore, pp 47-60.

Sugimoto, Y., Wigchert, S. C., Thuring, J. W. J., \& Zwanenburg, B. (1998). Synthesis of all eight stereoisomers of the germination stimulant sorgolactone. The Journal of Organic Chemistry, 63 (4), 1259-1267. 
Tasker AV, Westwood J.H., (2012). The U.S. witchweed eradication effort turns 50: a retrospective and lookahead on parasitic weed management. Weed Science 60:267-268.

Tenebe, V. A., \& Kamara, H. M. (2002). Effect of Striga hermonthica on the growth characteristics of sorghum intercropped with groundnut varieties. Journal of agronomy and crop science, 188 (6), 376-381.

Timko MP and Scholes JD, (2013). Host reaction to attack by root parasitic plants, in Parasitic Orobanchaceae, ed. by Joel DM, Gressel J and Musselman LJ. Springer, Berlin/Heidelberg, Germany, pp. 115-141.

Toomsan, B., et al. (1995). Nitrogen fixation by groundnut and soyabean and residual nitrogen benefits to rice in farmers' fields in Northeast Thailand. Plant and soil 175.1: 45-56.

Traore H, D., Yonli, D., Diallo and P., Sereme, (2011). Suicidal Germination of Striga hermonthica (Del.) Benth. by Cotton, Cowpea and Groundnut Genotypes in Burkina Faso. International Journal of Agricultural Research, 6: 49-57.

Ueno, K., Fujiwara, M., Nomura, S., Mizutani, M., Sasaki, M., Takikawa, H., \& Sugimoto, Y., (2011). Structural requirements of strigolactones for germination induction of Striga gesnerioides seeds. Journal of agricultural and food chemistry. 59(17), 9226-9231.

Van Ast, A., Bastiaans, L., and Kropff, M. J. (2000). A comparative study on Striga hermonthica interaction with a sensitive and a tolerant sorghum cultivar. Weed Research, 40(6), 479-493.

Van Ast, A., Bastiaans, L., and Katile, S., (2005). Cultural control measures to diminish sorghum yield loss and parasite success under Striga hermonthica infestation. Crop Protection 24(12), 1023-1034.

Van Mourik, T.A., Stomph, T.J. and Murdoch, A.J., (2011). Purple witchweed (Striga hermonthica) germination and seedbank depletion under different crops, fallow, and bare soil. Weed biology and management, 11 (2), pp.100-110.

Vasudeva Rao, M.J., Musselman, L.J., (1987). Host specificity in Striga spp. and physiological strains. In: Musselman, L.J. (Ed.), Parasitic Weeds in Agriculture, Vol. I, Striga. CRC Press, Boca Raton, FL, pp. $13 \pm 25$.

Westwood, J. H., Yoder, J. I., Timko, M. P., \& de Pamphilis, C. W. (2010). The evolution of parasitism in plants. Trends in Plant Science, 15(4), 227-235.

Wilson-Jones, K., (1955). Further experiments on witchweed control. II. The existence of physiological strains of Striga hermonthica. Empire Journal of Experimental Agriculture 23, 206-213.

Xie, X., Yoneyama, K. and Yoneyama, K., (2010). The strigolactone story. Annual review of phytopathology, 48. 93-117.

Xie, X., (2016). Structural diversity of strigolactones and their distribution in the plant kingdom. Journal of Pesticide Science, 41(4), pp.175-180.

Yoder, J. I., \& Scholes, J. D. (2010). Host plant resistance to parasitic weeds; recent progress and bottlenecks. Current opinion in plant biology, 13 (4), 478-484.

Yoneyama, K., Xie, X., Yoneyama, K., and Takeuchi, Y., (2009). Strigolactones: structures and biological activities. Pest management science, 65 (5), 467-470.

Zwanenburg B, Mwakaboko AS, Reizelman A, Anilkumar G, Sethumadhavan D., (2009). Structure and function of natural and synthetic signalling molecules in parasitic weed germination. Pest Management Science 65:478-49

Zwanenburg, B., \& Pospíšil, T. (2013). Structure and activity of strigolactones: new plant hormones with a rich future. Molecular plant, 6 (1), 38-62. 


\section{Supplementary tables}

Table S1: Logistic regression using strigolactones as independent variables (predictor), (A) Striga seed germination (\%) and (B) Striga plant per pot as dependent variables model summary (C) Non-significant model excluded independent variables. Data were squire root transformed prior to regression analysis.

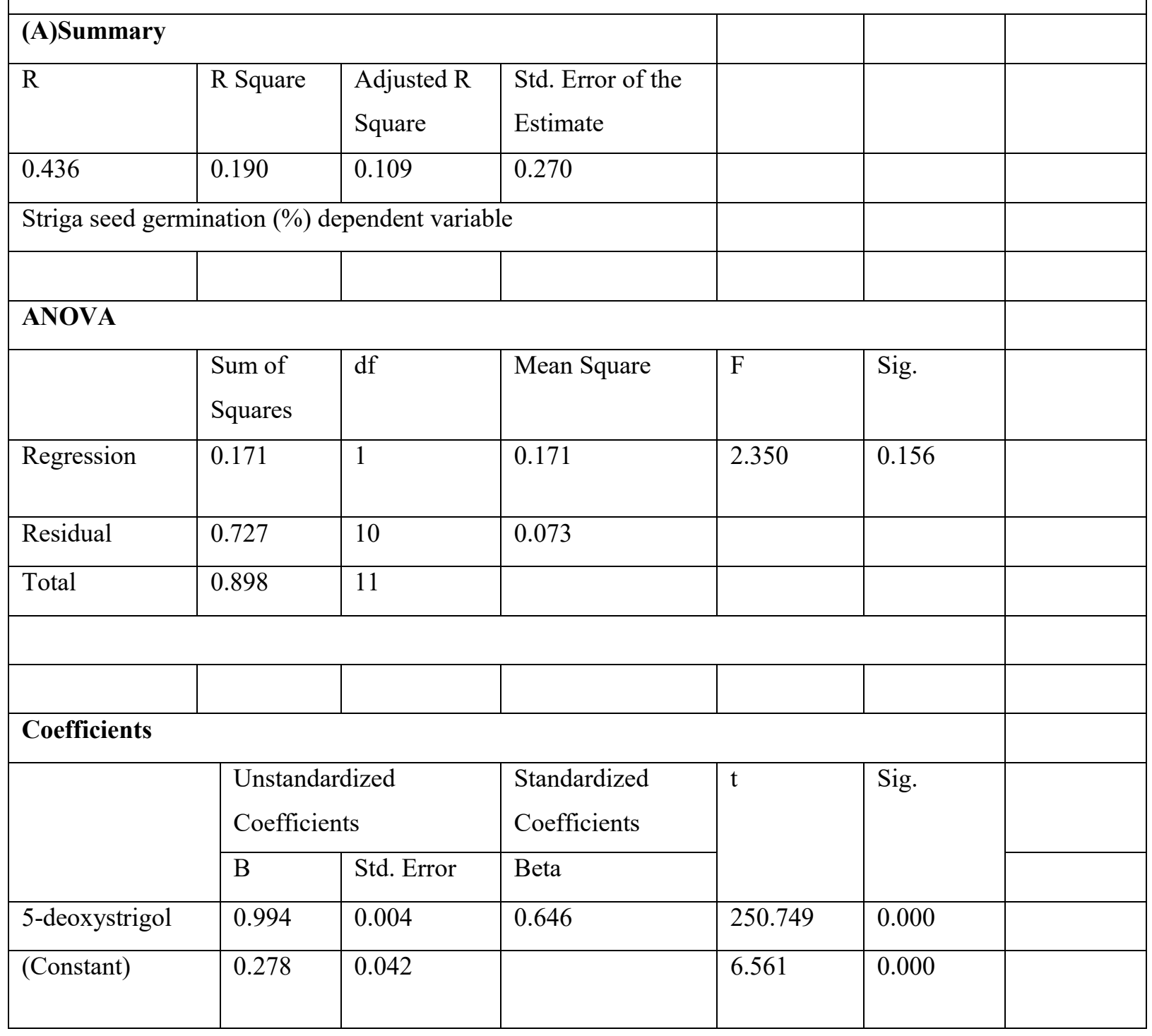




\section{Regression Striga emergence}

\begin{tabular}{|c|c|c|c|c|c|}
\hline \multicolumn{6}{|l|}{ (B) Summary } \\
\hline $\mathrm{R}$ & R Square & $\begin{array}{l}\text { Adjusted R } \\
\text { Square }\end{array}$ & $\begin{array}{l}\text { Std. Error of the } \\
\text { Estimate }\end{array}$ & & \\
\hline 0.697 & 0.486 & 0.435 & 0.236 & & \\
\hline \multicolumn{6}{|c|}{ Striga plant per pot dependent variable } \\
\hline \multicolumn{6}{|l|}{ ANOVA } \\
\hline & $\begin{array}{l}\text { Sum of } \\
\text { Squares }\end{array}$ & $\mathrm{df}$ & Mean Square & $\mathrm{F}$ & Sig. \\
\hline Regression & 0.528 & 1 & 0.528 & 9.462 & 0.012 \\
\hline Residual & 0.558 & 10 & 0.056 & & \\
\hline Total & 1.086 & 11 & & & \\
\hline & & & & & \\
\hline \multicolumn{6}{|l|}{ Coefficients } \\
\hline & \multicolumn{2}{|c|}{$\begin{array}{l}\text { Unstandardized } \\
\text { Coefficients }\end{array}$} & $\begin{array}{l}\text { Standardized } \\
\text { Coefficients }\end{array}$ & \multirow[t]{2}{*}{$\mathrm{t}$} & \multirow[t]{2}{*}{ Sig. } \\
\hline & $\mathrm{B}$ & Std. Error & Beta & & \\
\hline 5-deoxystrigol & 1.011 & 0.004 & 2.008 & 286.166 & 0.000 \\
\hline (Constant) & 0.312 & 0.042 & & 7.487 & 0.000 \\
\hline
\end{tabular}




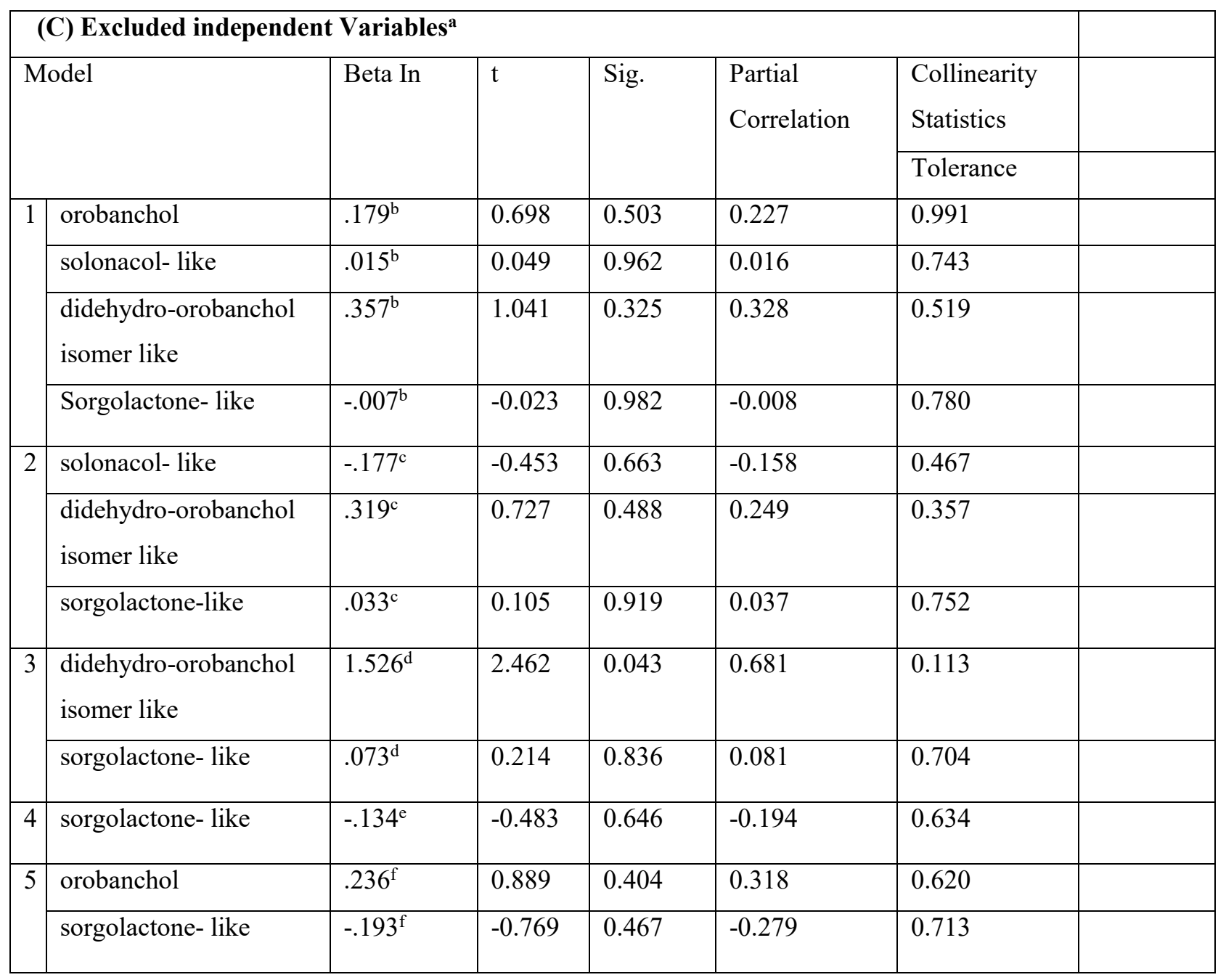

Table S2: Retention time and multiple reaction monitoring chennels used for detection of strigolactones present in the root exudates of inter and rotation crops.

\begin{tabular}{lll}
\hline Compound & RT. min & MRM channels $\boldsymbol{m} / \boldsymbol{z}$ \\
\hline 5-Deoxystrigol & 8.02 & $331>216,331>33$ and $331>97$ \\
orobanchol & 4.61 & $347>233,347>205$ and $347>97$ \\
orobanchyl acetate & 7.2 & $347>233$ and $389>247$ \\
solanacol-like & 3.6 & $343>183$ and $343>96$ \\
didehydro-orobanchol isomer like & 4.2 & $345>203$ and $345>97$ \\
sorgolactone-like & 7.6 & $317>97$ \\
sorgomol-like & 4.9 & $317>97$ and $347>317$. \\
\hline
\end{tabular}




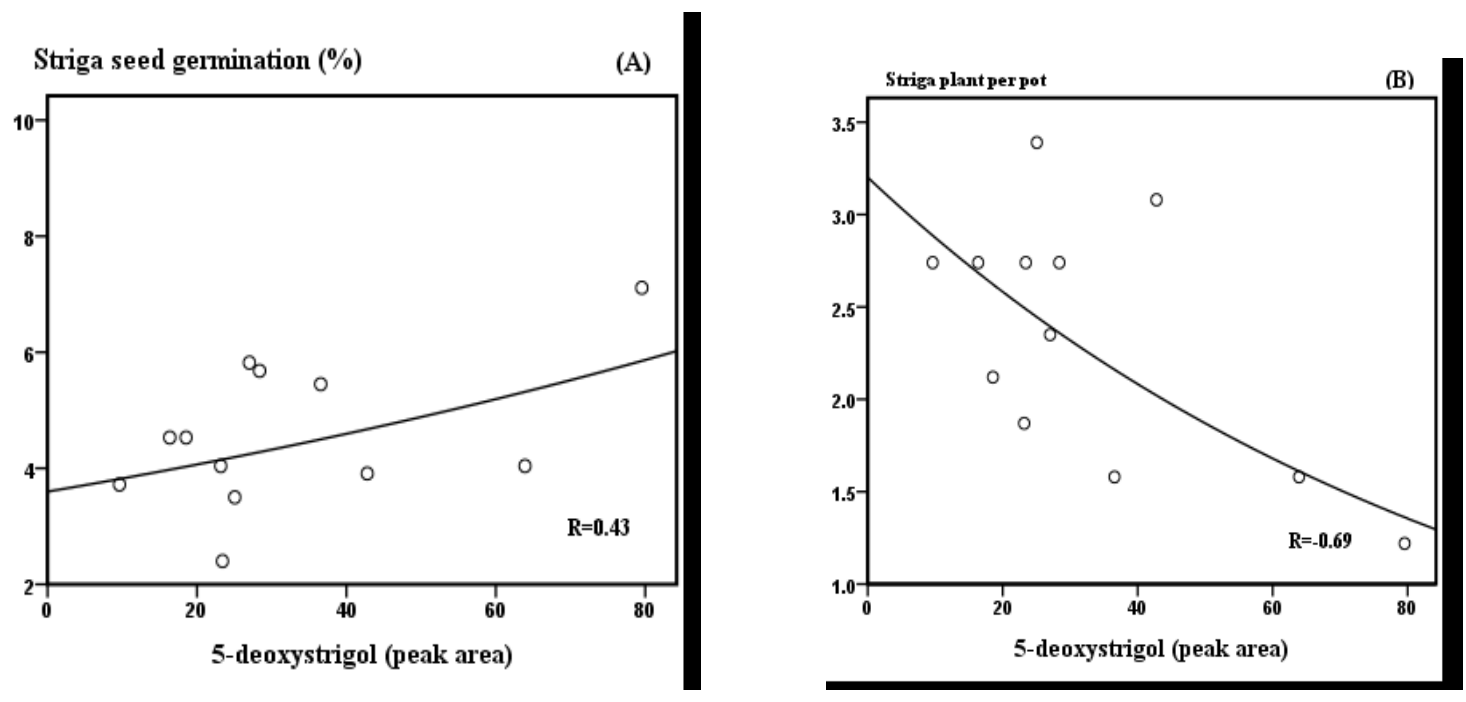

Fig. S1 Regression between the significant predictor 5-deoxystrigol peak areas and (A) Striga germination (\%) or (B) Striga emergence. Data presented as means of 3 replicates \pm SE. The line of best fit (Regression analysis $)$ is indicated $(P<0.05)$. 


\title{
Chapter 5
}

\section{Evaluation of field resistance to Striga hermonthica [Del.] Benth. in Sorghum bicolor [L.] Moench. The relationship with strigolactones}

This chapter was published as: Mohemed, N., Charnikhova, T., Bakker, E.J., van Ast, A., Babiker, A.G.T., Bouwmeester, H.J., 2016. Evaluation of field resistance to Striga hermonthica (Del.) Benth. in Sorghum bicolor (L.) Moench. The relationship with strigolactones. Pest Management Science 72: 2082-2090

\begin{abstract}
Background: Significant losses in sorghum biomass and grain yield occur in sub-Saharan Africa due to infection by the root parasitic weed Striga hermonthica [Del.] Benth. One strategy to avoid these losses is to adopt resistant crop varieties. To further delineate the role of germination stimulants in resistance we conducted a field experiment employing six sorghum genotypes, in eastern Sudan, and in parallel analysed the strigolactone levels in the root exudates of these genotypes under controlled conditions in Wageningen.
\end{abstract}

Results: The root exudates of these genotypes displayed large differences in strigolactone composition and Striga germination inducing activity. Korokollow, Fakimustahi and Wadfahel exuded the highest amounts of 5-deoxystrigol. Fakimustahi was by far the highest sorgomol producer and Wadbaco and SRN39 produced the highest amount of orobanchol. The concentration of 5-deoxystrigol in the root exudate showed a significant positive correlation with in vitro Striga germination and was positively associated with Striga infection in the field experiments, while orobanchol, on the other hand, was negatively associated with Striga infection in the field experiments.

Conclusion: For the first time a close association is reported between strigolactone levels, analysed under laboratory conditions, and Striga infection in the field in sorghum genotypes. These genotypes may be used to further study this resistance mechanism and 
for the introgression of the low germination trait in other sorghum varieties to breed for a strigolactone composition with low stimulant activity. The use of such improved varieties in combination with other Striga management tools could possibly alleviate the current Striga problem in the African continent.

\section{Introduction}

Sorghum is the most important cereal crop in the semi-arid parts of the world where it is the staple food crop for most of the population. In sub-Saharan Africa, the hemi-parasitic flowering weed, Striga hermonthica, is the major biotic constraint for sorghum production in countries including Botswana, Burkina Faso, Eritrea, Ethiopia, Kenya, Mali, Mozambique, Niger, Nigeria, Senegal, Sudan and Tanzania resulting in significant yield losses reaching up to $100 \%$ under heavy infestations. ${ }^{1-4}$

Copious seed production and prolonged viability of seeds make Striga a difficult weed to control. ${ }^{5,6}$ The lifecycle of Striga is strictly synchronised with the development of its host.

7 The first step in this lifecycle encompasses germination in response to host-derived, germination stimulants. ${ }^{8,9}$ Subsequent to germination a haustorium, with which Striga attaches to and penetrates the host roots and establishes a connection with the vascular system, is initiated in response to a second host-derived signal. ${ }^{10}$ The possibility to control Striga using cultural measures such as crop rotation, date and method of sowing, irrigation, trap cropping, hand-pulling, herbicides, fertilizers and suicidal germination stimulants has been investigated by many researchers. ${ }^{11-14}$ Currently, integrated control packages based on the use of resistant genotypes in combination with cultural and/or chemical control methods seem to be the best option for Striga management. ${ }^{15-17}$

However, resistance against these parasitic weeds is a complex phenomenon that is also dependent on the interaction between the parasite, the host and the environment. Striga resistance is broadly categorized as pre- and post-attachment resistance. 9,10,18 Preattachment resistance concerns disruption of one or more of the events starting at germination to haustorium attachment, while post-attachment resistance encompasses interruption of root penetration and/or subsequent developmental stages. In sorghum several mechanisms of resistance have been described, ranging from low germination 
stimulation to post-attachment resistance due to mechanical barriers, hypersensitive response, antibiosis and occlusion and sealing off of the host vessels. ${ }^{9,10,19,20}$

Of these mechanisms, low germination stimulation $(\lg s)$ is the best documented. ${ }^{18,21,22}$ It is relatively easy to assay ${ }^{23}$ and has the advantage of decreasing infection and reducing further seed bank build-up. ${ }^{24}$ Indeed, in the past decade or so several sorghum varieties with Striga resistance based on low germination stimulation have been introduced, such as SRN39 and IS9830 in Sudan, and Abshir, Gobiye and Birhan in Ethiopia. ${ }^{25,26}$ In an attempt to create more durable resistance, a research program led by Purdue University further dissected the Striga infection cycle into key events including germination, haustorium formation, root penetration, establishment of a connection with the host vascular system and further Striga development. Assays were developed for these events and subsequently used to identify resistance sources in breeding materials or wild relatives. ${ }^{19,27,28}$ These resistances can then be combined in farmer-preferred genetic backgrounds facilitated by DNA-based markers. An example of the validity of this approach is the identification of the low germination stimulation QTL in Striga resistant sorghum SRN39. ${ }^{29}$ The authors demonstrated that the low germination stimulation SSR markers were also present in other Striga resistant genotypes, e.g. Tetron and IS9830. ${ }^{29}$ In addition to low germination stimulation based resistance, also a stable broad spectrum QTL for post attachment field resistance in line N13 and molecular markers flanking these QTLs have recently been published. ${ }^{30}$ These findings broaden the opportunity to transfer QTLs for Striga resistance from lines such as N13 and SRN39 into susceptible genotypes with preferred agronomic qualities through Marker Assisted Selection (MAS). ${ }^{31}$ The first products of MAS for Striga resistance were released for cultivation in Sudan (lines T1BC3S4, AG6BC3S4, $\mathrm{AG} 2 \mathrm{BC} 3 \mathrm{~S} 4$ and W2BC3S4) combining post attachment Striga resistance with farmer preference. ${ }^{32}$ These lines still lack the low germination stimulation trait. Using MAS pyramiding of different resistance mechanisms into preferred genotypes is expected to make resistance more robust and improve its durability. ${ }^{33}$

Striga resistance is occasionally described as a mechanism that ensures lower field infection and allows satisfactory or higher yields than with (fully) susceptible genotypes. 
30,34 Tolerance, which refers to the host's ability to endure the negative impact of Striga on yield once it is infected, is also an important trait. ${ }^{35,36}$ Van Ast et al. ${ }^{37}$, for example, reported that under Striga infection a local Malian sorghum landrace (Tiémarifing) displayed tolerance; it had delayed Striga infection and maintained a normal photosynthetic rate despite the fact that Striga infection did occur, but later than in a non-tolerant genotype. This resulted in a less affected biomass and better grain yield than in the non-tolerant control. In the absence of complete immunity to Striga, the line between resistance and tolerance is thin and each genotype likely combines a certain degree of resistance and tolerance. Therefore, the preservation of yield under Striga infection is the result of the combined effects of resistance and tolerance. ${ }^{34}$ It would be good if researchers would consider both traits individually to maximise the exploitation of these mechanisms in crop yield improvement. ${ }^{38}$

In 1991, ICRISAT provided accessions SRN39 and IS9830 as varieties exhibiting field resistance due to low germination stimulant production. ${ }^{39}$ However, despite an adequate level of resistance, SRN39 and IS9830 were not adopted on a commercial scale as they are liable to pest damage. Furthermore, farmers favour Feteritas type sorghums for high value food and feed quality. ${ }^{40}$ Resistance to the parasite per se thus is not sufficient for adoption of a variety as adaptability to the environment and farmer preference have to be taken into account.

In the present study we focused on pre-attachment resistance in sorghum. Germination is the most important component of pre-attachment resistance and, as described above, low germination has successfully been introduced into sorghum varieties to obtain Striga resistance. ${ }^{8,29}$ However, the underlying mechanisms of this resistance have not been identified. As germination of Striga is induced by strigolactones present in the root exudates of its hosts, ${ }^{41}$ we decided to study strigolactone production in a number of sorghum genotypes reported to differ in Striga field resistance. Strigolactone analysis was carried out under controlled conditions in Wageningen, the Netherlands while Striga infection of these same genotypes was studied under field conditions in Sudan. The study was conducted at two sites in the Gadarif State. The latter constitutes the largest 
mechanized rainfed sorghum production centre in Sudan. We used local Sudanese landraces Korokollow, Mogud, and Wadbaco, reported to be drought tolerant and less affected by Striga, and Wadfahel and Fakmustahi, reported to be susceptible to Striga and used SRN39, as a Striga resistant control. ${ }^{42}$

\section{Materials and Methods}

\section{Germplasm and chemicals}

Seeds of 6 sorghum genotypes, representing two groups differing in morphology and susceptibility or sensitivity to Striga hermonthica infection, were provided by the Agricultural Research Corporation (ARC), Sudan. Seeds of Striga hermonthica used in the field trials and in the germination bioassays were collected from a sorghum field at $\mathrm{Abu}$ Naama Research Station, ARC, Sudan in 2010. Striga seed germinability was about 6070\%. Strigolactone standards were provided by Koichi Yoneyama (Weed Science Center, Utsunomiya University, Japan) and Tadao Asami (Applied Biological Chemistry the University of Tokyo, Japan).

\section{Experimental sites}

The field trials were conducted during the rainy season, July -September 2012 in a wet and a dry site in Gadarif State in eastern Sudan, which encompasses the largest rainfed sorghum producing area in the country. The first site was at Tawareet, a village located $75 \mathrm{~km}$ south of Gadarif town (latitude $13^{\circ} 44^{\prime} \mathrm{N}$, longitude $35^{\circ} 77^{\prime} \mathrm{E}$, elevation $604 \mathrm{~m}$ above sea level) in the relatively wet Doka district, with a cumulative rainfall, measured in the field during the trials, of $957 \mathrm{~mm}$. The second site was at Gadarif Agricultural Research Station Farm (Kilo-6), about $12 \mathrm{~km}$ west of Gadarif town (latitude $14^{\circ} 03^{\prime} \mathrm{N}$, longitude $32^{\circ} 22^{\prime} \mathrm{E}$, elevation $539 \mathrm{~m}$ above sea level) in a relatively dry district, with a cumulative rainfall, measured in the field during the trials, of $423 \mathrm{~mm}$. The soil at both sites is a vertisol, with very low organic matter and low nitrogen content. Both sites were former farmer fields under sorghum cultivation and are naturally infested by Striga. At the Kilo- 6 site, where 
Striga infection was low, additional Striga seeds were added at planting, as explained below.

\section{Experimental design, plot sizes and field preparation}

In both locations, the trials were laid out as a randomized complete block design with six replications. Before sowing, the land was ploughed twice, and the crop was sown on flat soil in rows $80 \mathrm{~cm}$ apart with $20 \mathrm{~cm}$ between plants. The two sorghum groups, the late maturing genotypes Fakimustahi, Wadfahel and Mogud and the early maturing genotypes Wadbaco, Korakollow, and the Striga resistant check SRN39 were sown in both sites. In both sites, sorghum was directly sown at approximately 6 seeds per planting hole, and thinned to 2-3 plants per hole 20 days after sowing (DAS). The trials were hand-weeded at 21 and 30 DAS, to remove all weeds except Striga, and as needed afterwards. Each subplot, comprising 5 rows and representing one cultivar, measured $3.20 \times 4.40 \mathrm{~m}\left(14.08 \mathrm{~m}^{2}\right)$. The sub-plots were separated by one open row of $2 \mathrm{~m}$ to avoid neighbour effects and to allow easy access. The blocks were separated by a $1 \mathrm{~m}$ open row. Total field size, including passages was $19.2 \times 38 \mathrm{~m}\left(729.6 \mathrm{~m}^{2}\right)$. At Kilo-6, each plant received about $5000 \mathrm{Striga}$ seeds mixed in $5 \mathrm{~g}$ clay that was placed in the planting holes at sowing and incorporated into the upper 5-10 $\mathrm{cm}$ of the soil. At Tawareet, based on experience, the Striga seed bank was expected to be high enough and therefore no additional Striga seeds were added to the plots. The crop was sown on 22 July at Tawareet and on 23 July at Kilo-6. The cropping season in the area runs from the end of June to December and the average temperature in this period is $26.1^{\circ} \mathrm{C}$.

\section{Experimental measurements}

Three weeks after sowing, emergence of Striga was observed in $50 \%$ of the plots. From then on, the total number of emerged Striga plants, in each sub-plot, was counted at 2-wk intervals. These data enabled the assessment of the total number of emerged Striga plants over the entire growing period for each genotype. At harvest, from each sub-plot, the emerged Striga plants were collected, dried and weighed for determination of Striga dry biomass. Measurements ended at harvest based on maturity of the sorghum genotype. At 
harvest, sorghum plants from the two inner rows were cut just above the ground, and heads and stalks separately dried and weighed.

\section{Production and analysis of root exudates}

For collection of root exudates, sorghum was grown in a climate room in Wageningen, the Netherlands. The experiment was laid out as a complete randomized block design with four replications. Pre-germinated sorghum seeds of each genotype were planted in a $3 \mathrm{~L}$ plastic pot filled with $1.5 \mathrm{~L}$ sand. After one week, the seedlings were thinned to five per pot. Half strength modified Hoagland's nutrient solution containing $\mathrm{NH}_{4} \mathrm{NO}_{3}(5.6 \mathrm{mM}), \mathrm{K}_{2} \mathrm{HPO}_{4}$ (0.4 mM), $\mathrm{MgSO}_{4}(0.8 \mathrm{mM}), \mathrm{FeSO}_{4}(0.18 \mathrm{mM}), \mathrm{CaCl}_{2}(1.6 \mathrm{mM}), \mathrm{K}_{2} \mathrm{SO}_{4}(0.8 \mathrm{mM}), \mathrm{MnCl}_{2}$ (0.0045 mM), $\mathrm{CuSO}_{4}(0.0003 \mathrm{mM}), \mathrm{ZnCl}_{2}(0.0015 \mathrm{mM})$ and $\mathrm{Na}_{2} \mathrm{MoO}_{4}(0.0001 \mathrm{mM})$ was applied to each pot (500 $\mathrm{mL}$ at $48 \mathrm{~h}$ intervals). The plants were grown in a climate room with artificial lighting at $450 \mu \mathrm{mol} \mathrm{m} \mathrm{m}^{-2} \mathrm{~s}^{-1}$ and controlled conditions $\left(28^{\circ} \mathrm{C}\right.$ (D) $10 \mathrm{~h}$ and $25^{\circ} \mathrm{C}(\mathrm{N}) 14 \mathrm{~h}$ at $70 \%$ relative humidity) for four weeks. In the 5 th week, phosphorus deficiency was created in each pot to increase strigolactone production. Hereto, 3 litres phosphorus deficient nutrient solution (half strength Hoagland's nutrient solution minus phosphate) was added to each pot and allowed to drain freely through the holes in the bottom of the pot to remove phosphorus from the sand. The plants were kept under $\mathrm{P}$ deficiency for one week to increase strigolactone production. ${ }^{43}$ In the 6 th week the same draining with $3 \mathrm{~L}$ of phosphorus deficient nutrient solution was again done to remove any accumulated strigolactones. Finally, $48 \mathrm{~h}$ later, root exudates were collected in $1 \mathrm{~L}$ plastic bottles by passing $1.5 \mathrm{~L}$ nutrient solution without phosphate through each pot. The collected root exudates were then run through an SPE C18 column (500 mg) and strigolactones were eluted with $6 \mathrm{~mL}$ acetone.

The strigolactones, 5-deoxystrigol, sorgomol and orobanchol were identified and quantified using ultra performance liquid chromatography tandem mass spectrometry (UPLC-MS/MS) as previously described. ${ }^{43}$ Because orobanchol has not been reported before in sorghum (possibly due to different strigolactone collection techniques used by others), its identification was carefully confirmed - by MS/MS and co-injection - using authentic standards obtained as described before. ${ }^{44}$ The samples were analysed by a Waters 
Xevo triple quadrupole tandem mass spectrometer (Waters, Milford, MA, USA) equipped with an electrospray ionization ${ }^{44}$ source and coupled to an Acquity UPLC system (Waters, USA). Multiple reaction monitoring (MRM) was used for quantification of the strigolactones. Data acquisition and analysis were performed using Mass Lynx 4.1 (TargetLynx) (Waters). The biological activity of the root exudate of each genotype was studied using a Striga germination bioassay with pre-conditioned Striga seeds as described previously. ${ }^{46}$

\section{Statistical analysis}

The statistical package SAS (version 9.2) was used for ANOVA and Pearson's correlation analyses. Duncan's honest significant difference test was then performed to establish the significance of differences. To meet the assumptions of the analysis of variance, data involving strigolactone peak area and number of attached Striga were subjected to logarithmic transformation prior to analysis.

\section{Results}

\section{Striga emergence}

To assess the level of field resistance to Striga, emerged Striga plants were counted at 2wks intervals. Around 45-65 DAS large numbers of Striga plants were observed vigorously growing on Fakimustahi, Wadfahel and Korokollow especially at the wet site (Figure 1), whereas much less numbers of Striga plants were observed on Wadbaco, Mogud and SRN39. Analysis of variance showed highly significant differences between the genotypes. Emerged Striga numbers on Korokollow, Wadfahel and Fakimustahi at both sites were significantly higher than on Wadbaco, Mogud and SRN39 (Figure 1). Wadbaco and Mogud showed identical emerged Striga numbers and ranked intermediate in level between Wadfahel and Fakimustahi on one hand and the resistant check SRN39 on the other hand, at both sites (Figure 1). 


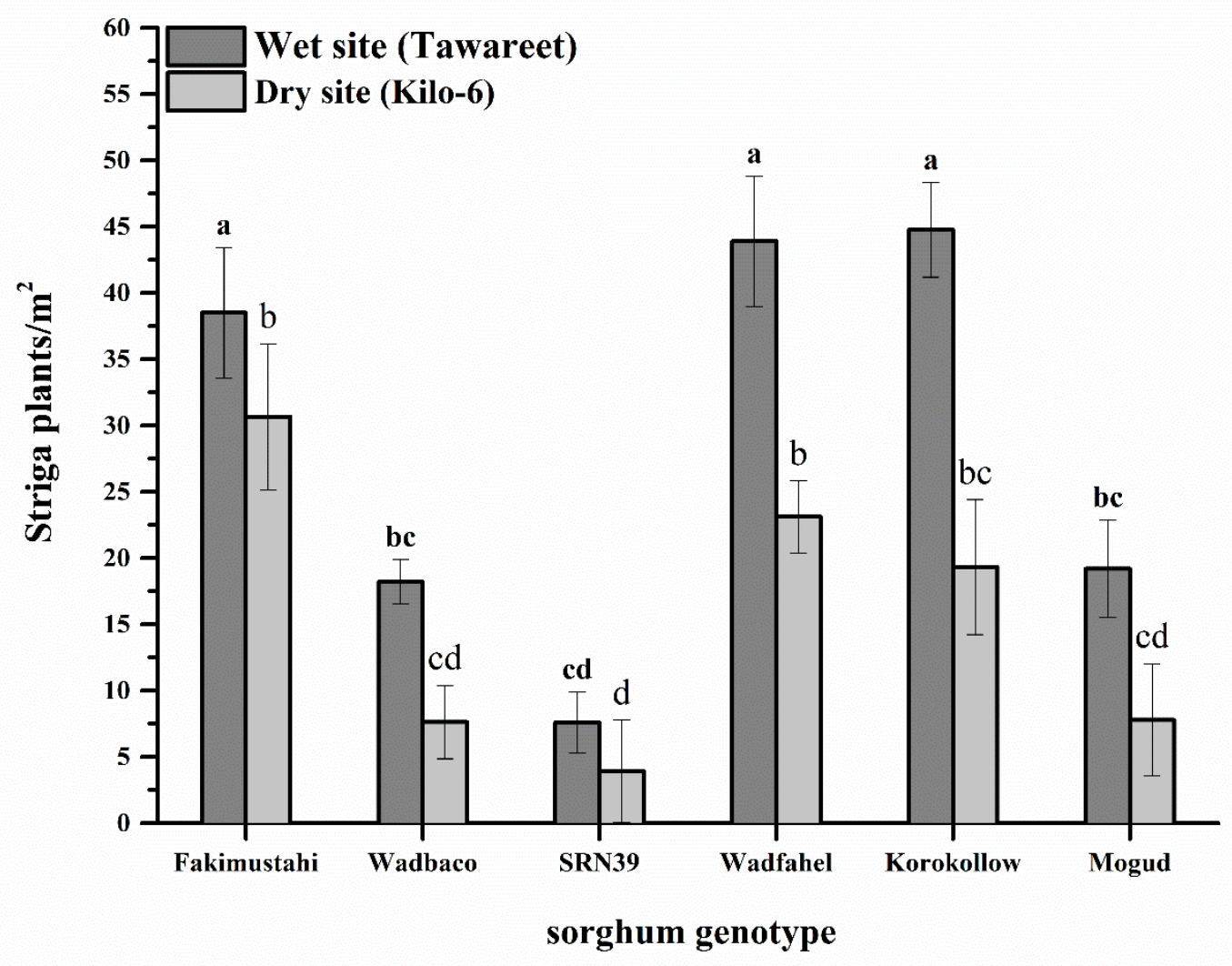

Fig. 1 Striga emergence at Tawareet (wet site) and Kilo-6 (dry site). Bars represent means of total Striga numbers emerged over the entire growing period \pm standard error $(n=6)$ for each genotype. The significance of a genotypic difference per site was determined by a one-way ANOVA for all genotypes and the letters indicate the different significance groups after Duncans' pairwise comparisons $(P<0.05)$.

\section{Striga dry biomass}

In the wet site Wadfahel, Fakimustahi and Korokollow supported the highest Striga biomass followed, in descending order, by Mogud, Wadbaco and SRN39 $(P<0.0001)$ (Table 1). In the dry site Wadfahel and Fakimustahi supported the highest Striga dry biomass followed in descending order by Korokollow, Wadbaco, Mogud and SRN39 (Table 1). Irrespective of the site a strong positive linear correlation was observed between the number of emerged Striga plants and Striga dry biomass (Fig. 2). Based on the number of emerged Striga and Striga biomass, the three most susceptible genotypes are Korokollow, Wadfahel, and Fakimustahi and the three most resistant genotypes are Mogud, Wadbaco and SRN39 (Fig. 1 and Table 1). 
Table 1 Means of total Striga dry biomass $(\mathrm{g} / \mathrm{m} 2)$ of different sorghum genotypes over the entire growing period \pm standard error $(n=6)$ for each genotype. The significance of a treatment effect was determined by a one-way ANOVA for all genotypes and the letters indicate the different significance groups after Duncans' pairwise comparisons $(\mathrm{P}<0.05)$.

\begin{tabular}{|c|c|c|c|}
\hline \multirow{2}{*}{\multicolumn{2}{|c|}{$\begin{array}{l}\text { Wet site (Tawareet) } \\
\text { Striga biomass }(\mathrm{g} / \mathrm{m} 2)\end{array}$}} & \multicolumn{2}{|c|}{ Dry site (Kilo-6) } \\
\hline & & \multicolumn{2}{|c|}{ Striga biomass $(\mathrm{g} / \mathrm{m} 2)$} \\
\hline Wadfahel & $89.3 \mathrm{a}$ & Fakimustahi & $39.8 \mathrm{~cd}$ \\
\hline Fakimustahi & $67.0 \mathrm{~b}$ & Wadfahel & $26.1 \mathrm{cde}$ \\
\hline Korokollow & $41.8 \mathrm{c}$ & Korokollow & $21.8 \mathrm{ef}$ \\
\hline Mogud & $28.5 \mathrm{cde}$ & Wadbaco & $11.5 \mathrm{ef}$ \\
\hline Wadbaco & $23.3 \mathrm{def}$ & Mogud & $7.3 \mathrm{f}$ \\
\hline SRN39 & $6.5 \mathrm{f}$ & SRN39 & $5.1 \mathrm{f}$ \\
\hline
\end{tabular}

(A)

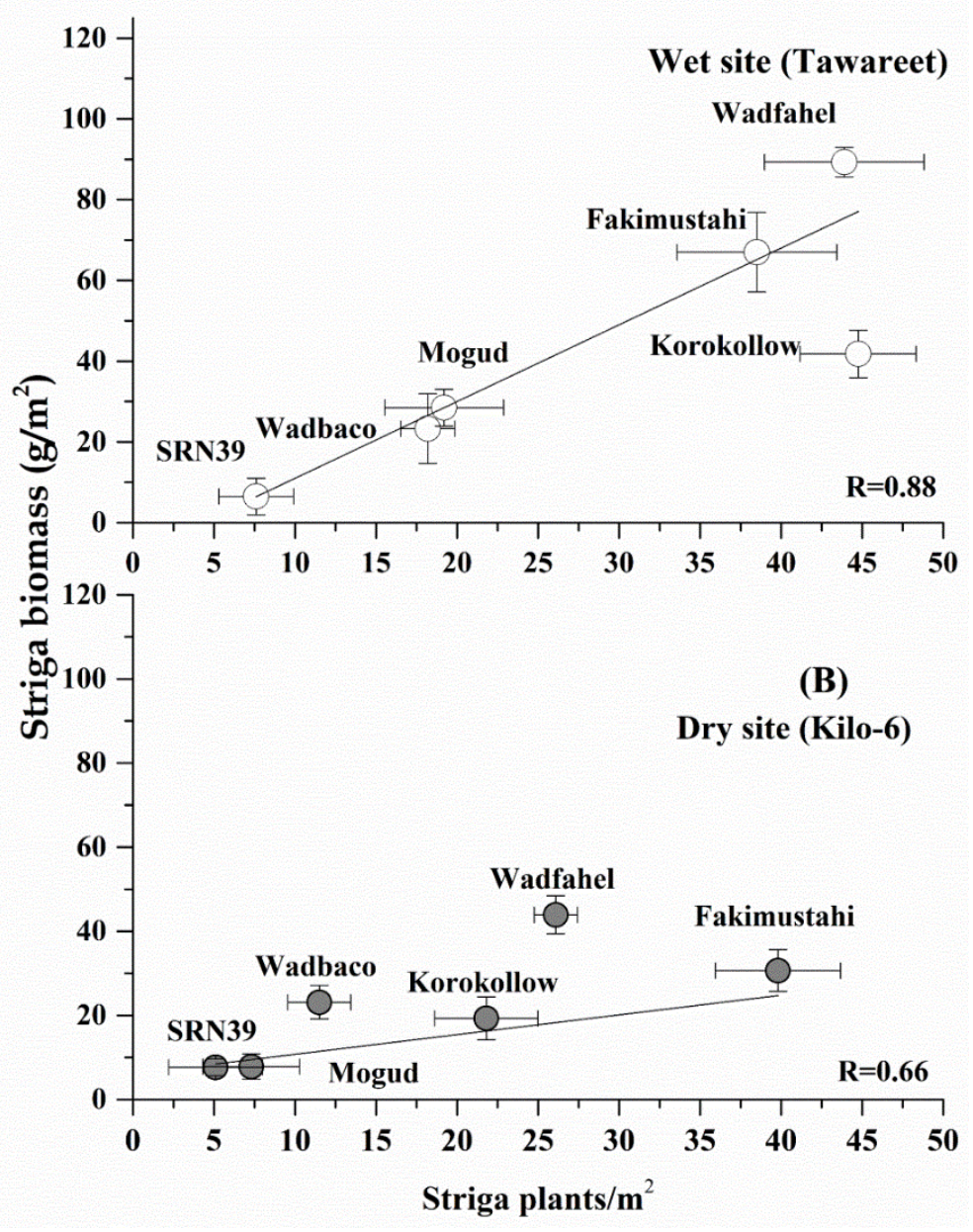

Fig. 2 Relationship between Striga numbers and Striga biomass per genotype. Lines represent the best fit as determined by regression analysis at the wet (A) and dry site (B). 


\section{Relationship between strigolactone concentrations and field resistance}

UPLC-MS/MS analysis showed that the strigolactone composition and concentration in the root exudate varied strongly between sorghum genotypes. Korokollow, Fakimustahi and Wadfahel exuded the highest amounts of 5-deoxystrigol (Fig 3A). Mogud exuded an intermediate amount, while Wadbaco and SRN39 exuded the lowest amount. Fakimustahi and Wadfahel were the highest sorgomol producers, whereas Wadbaco and particularly SRN39 were the highest orobanchol producers (Fig. 3). 

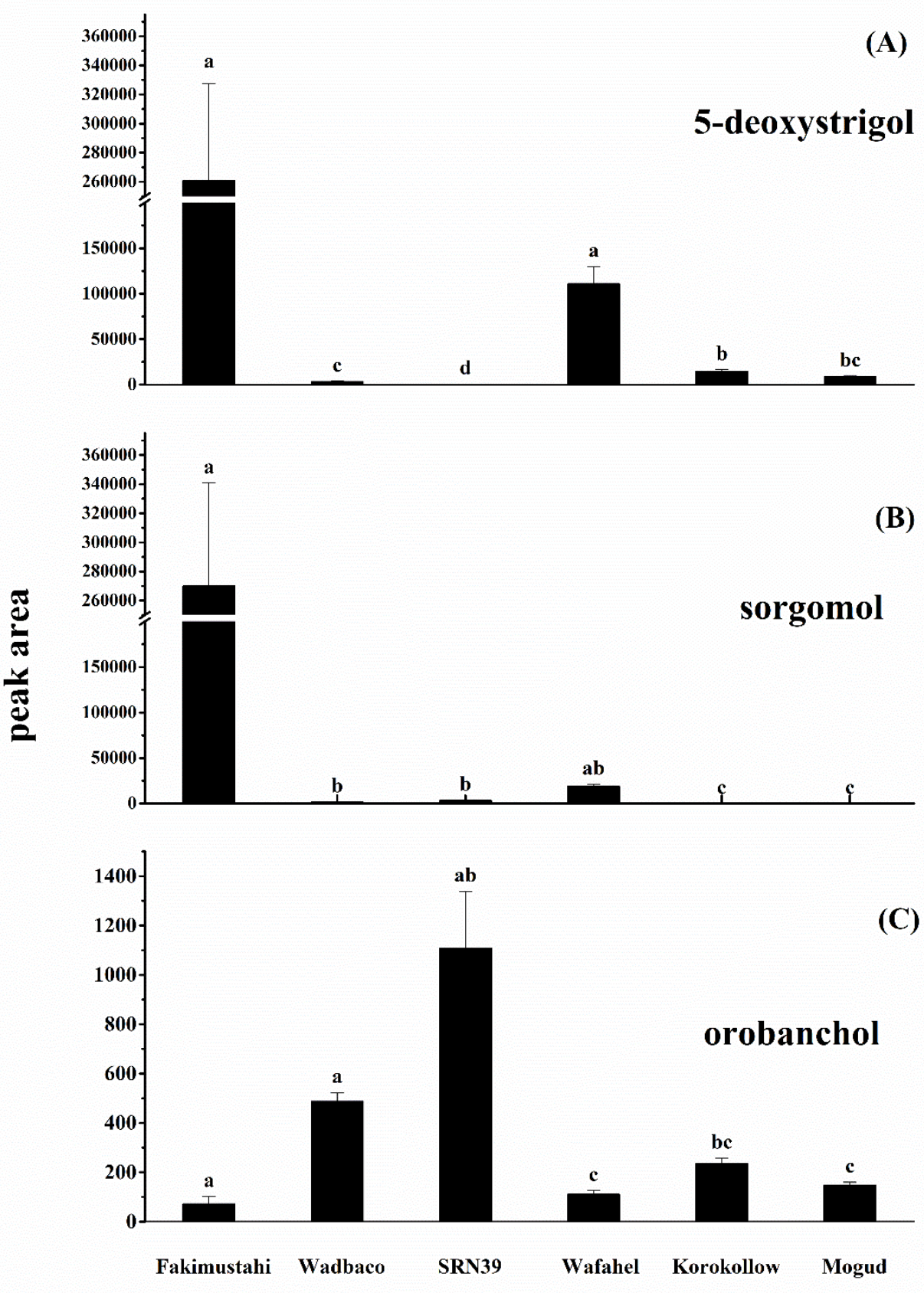

sorghum genotype

Fig 3: Strigolactone composition and concentration (peak area) in root exudates of sorghum genotypes. Strigolactone levels were analysed using liquid chromatography coupled with tandem mass spectrometry (LC-MS/MS). (A), concentration of 5-deoxystrigol; (B), concentration of sorgomol and (C), concentration of orobanchol in root exudates of different sorghum genotypes. Bars represent means \pm standard error $(n=4)$. Least significant differences of means at $P=0.05$ were determined by ANOVA. Letters indicate the different significance groups after Duncans' pairwise comparisons $(P<0.05)$. 
Germination bioassays undertaken to investigate the relationship between strigolactone composition and concentration in the root exudates and Striga germination showed that Fakimustahi, Korokollow and Mogud induced the highest germination (30-50\%), followed closely by Wadfahel, while Wadbaco and SRN39 induced significantly less germination compared with the other genotypes (Fig. 4).

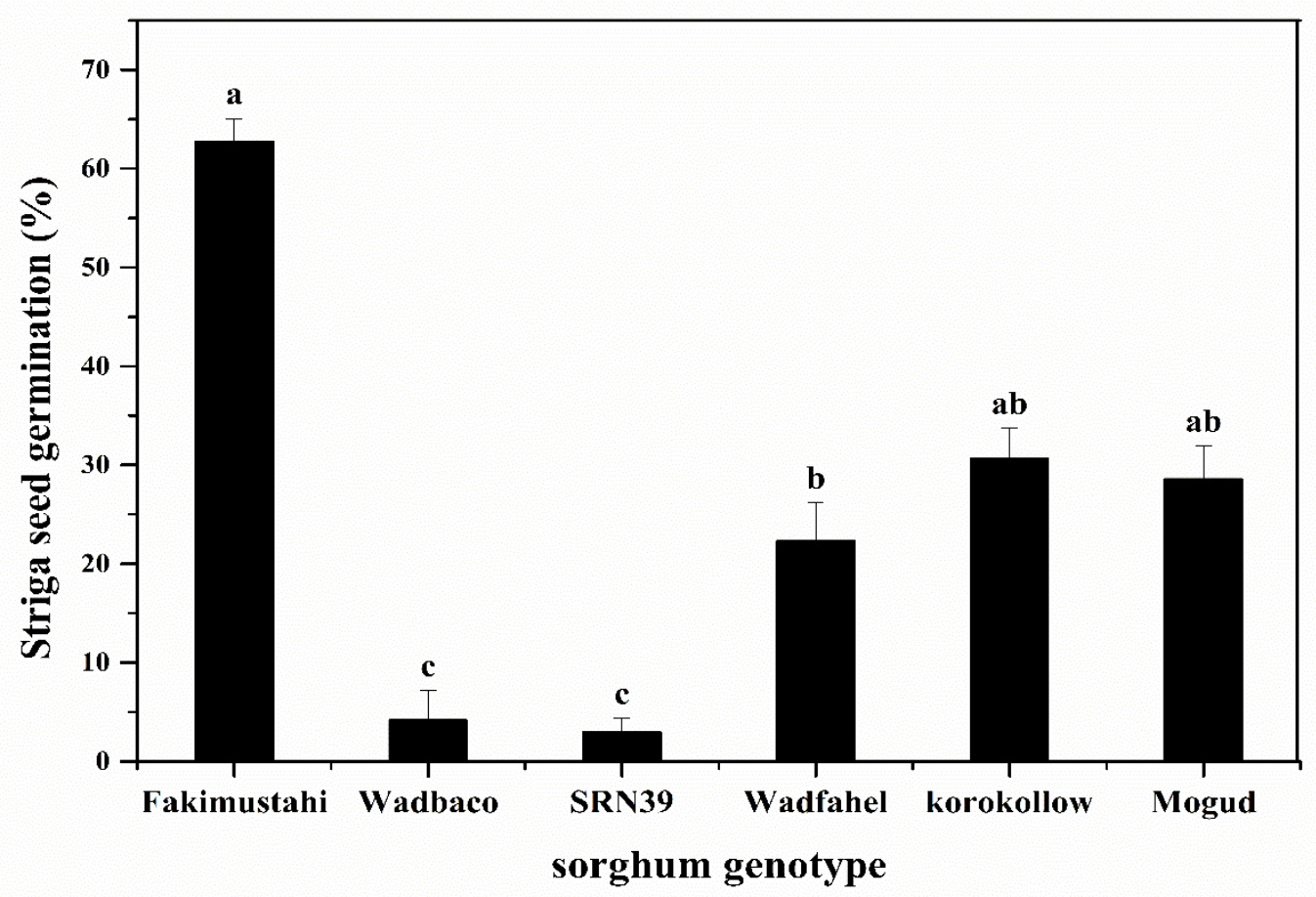

Fig. 4: Germination of pre-conditioned Striga seeds as induced by root exudates collected from different sorghum genotypes. Bars represent means \pm standard error $(n=5)$. Least significant differences of means at $P=$ 0.05 were determined by ANOVA. Letters indicate the different significance groups after Duncans' pairwise comparisons $(P<0.05)$.

To establish which of the strigolactones contributes most to the observed germination, Pearson correlation coefficients were determined between the individual strigolactones and germination (Table 2). The 5-deoxystrigol amounts in the root exudates correlated significantly with germination $(\mathrm{r}=0.63 ; \mathrm{P}<0.01)$ while sorgomol showed a non-significant positive correlation and orobanchol a non-significant negative correlation. This suggests that production of high amounts of 5-deoxystrigol results in high Striga germination, which 
is consistent with the high Striga emergence observed in Fakimustahi, Wadfahel and Korokollow (Fig. 1). The low production of 5-deoxystrigol may account for the low Striga emergence observed in Wadbaco and SRN39 (Fig.1).

Interestingly, there was a similar relationship between the strigolactone concentrations in the root exudate of the sorghum genotypes, grown in a greenhouse in Wageningen, and Striga infection on all genotypes grown in the field in Sudan (Fig. 5). There was a positive relationship between the peak areas of 5-deoxystrigol and sorgomol with the number of emerged Striga plants $\left[\mathrm{R}^{2}=0.76\right.$ and 0.54 , respectively for the dry site (Fig. $5 \mathrm{~A}-\mathrm{B}$ ) and $\mathrm{R}^{2}$ $=0.28$ and 0.22, respectively, for the wet site (data not shown)] and with Striga biomass $\left[R^{2}=0.83\right.$ and 0.78 , respectively, for the dry site (Fig. 5D-E) and $R^{2}=0.28$ and 0.11 , respectively, for the wet site (data not shown)]. The peak area of orobanchol displayed a negative relationship with the number of emerged Striga plants $\left(\mathrm{R}^{2}=0.50\right)$ and Striga biomass $\left(\mathrm{R}^{2}=0.42\right)$ for the dry site (Fig. $5 \mathrm{C}, \mathrm{F}$ ) and also with the number of emerged Striga plants $\left(\mathrm{R}^{2}=0.59\right)$ and with Striga biomass $\left(\mathrm{R}^{2}=0.56\right)$ for the wet site (data not shown). 

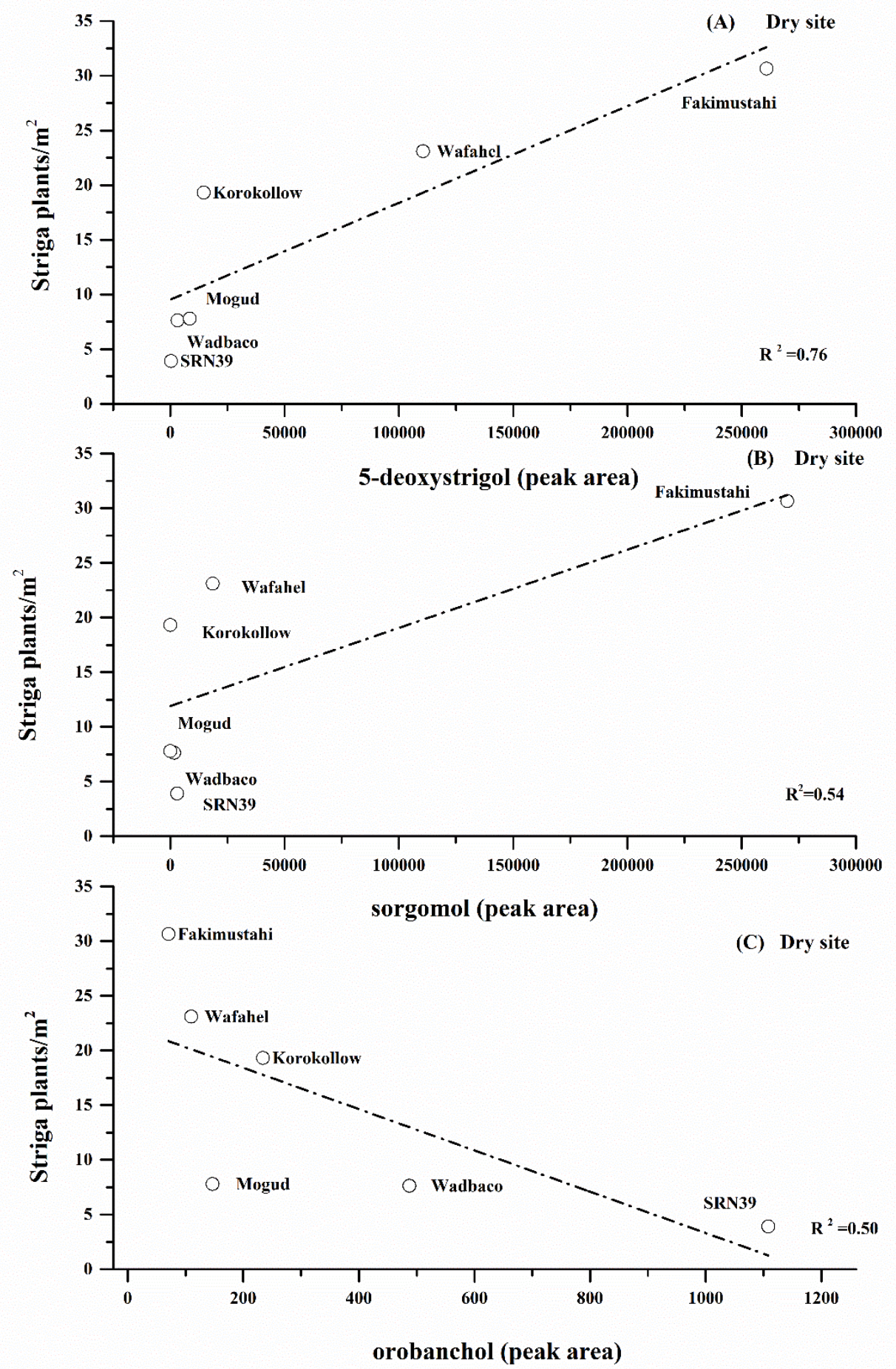

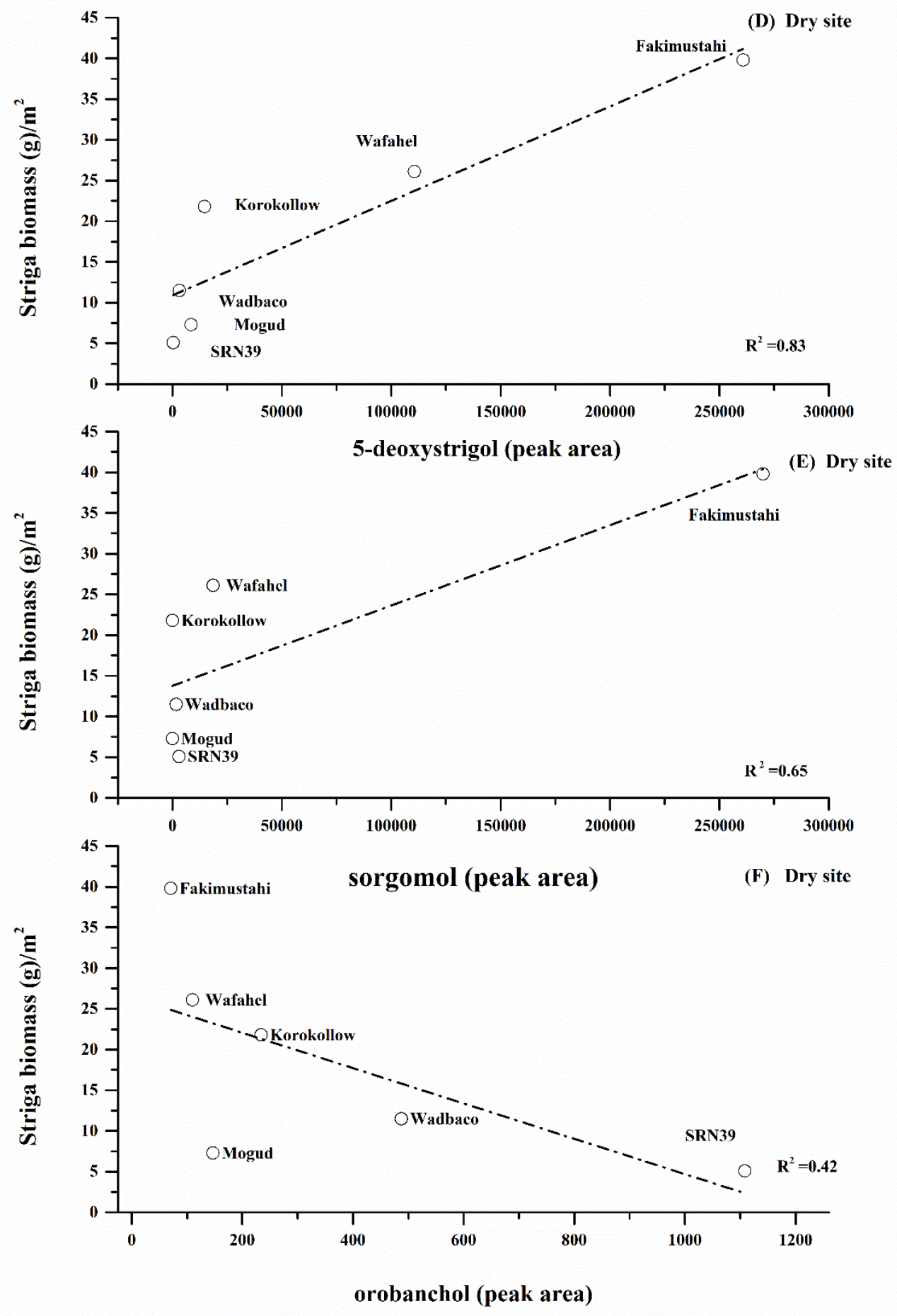

Fig. 5 Relation between strigolactone concentrations in the root exudates of sorghum genotypes, grown in a climate room in the Netherlands and Striga infection parameters on the dry site in the field in Sudan. 
Table 3 Means of sorghum biomass $\left(\mathrm{g} / \mathrm{m}^{2}\right)$ and grain yield $\left(\mathrm{g} / \mathrm{m}^{2}\right)$ for 6 sorghum genotypes grown at two different sites.

\begin{tabular}{|c|c|c|c|}
\hline \multicolumn{2}{|c|}{ Wet site (Tawareet) } & \multicolumn{2}{|c|}{ Dry site (Kilo-6) } \\
\hline \multicolumn{2}{|c|}{ sorghum biomass $\left(\mathrm{g} / \mathrm{m}^{2}\right)$} & \multicolumn{2}{|c|}{ sorghum biomass $\left(\mathrm{g} / \mathrm{m}^{2}\right)$} \\
\hline Mogud & $972.2 \mathrm{a}$ & Mogud & $266.7 \mathrm{bc}$ \\
\hline Wadbaco & 888.9 a & Wadbaco & $683.3 \mathrm{a}$ \\
\hline SRN39 & $672.2 \mathrm{~b}$ & SRN39 & $490.2 \mathrm{ab}$ \\
\hline Korokollow & $561.1 \mathrm{~b}$ & Korokollow & $427.8 \mathrm{bc}$ \\
\hline Fakimustahi & $361.1 \mathrm{c}$ & Fakimustahi & $166.7 \mathrm{c}$ \\
\hline Wadfahel & $277.7 \mathrm{c}$ & Wadfahel & $166.7 \mathrm{c}$ \\
\hline \multicolumn{2}{|c|}{ Wet site (Tawareet) } & \multicolumn{2}{|c|}{ Dry site (Kilo-6) } \\
\hline \multicolumn{2}{|c|}{ sorghum grain yield $\left(\mathrm{g} / \mathrm{m}^{2}\right)$} & \multicolumn{2}{|c|}{ sorghum grain yield $\left(\mathrm{g} / \mathrm{m}^{2}\right)$} \\
\hline Mogud & $131.1 \mathrm{a}$ & Mogud & $77.6 \mathrm{bc}$ \\
\hline Wadbaco & $130.6 \mathrm{a}$ & Wadbaco & $136.4 \mathrm{a}$ \\
\hline SRN39 & $102.4 \mathrm{a}$ & SRN39 & $68.7 \mathrm{c}$ \\
\hline Korokollow & $137.1 \mathrm{a}$ & Korokollow & $109.0 \mathrm{ab}$ \\
\hline Fakimustahi & $29.4 \mathrm{~b}$ & Fakimustahi & $9.7 \mathrm{~d}$ \\
\hline Wadfahel & $17.8 \mathrm{~d}$ & Wadfahel & $17.7 \mathrm{~b}$ \\
\hline
\end{tabular}

*The significance of the treatment effects were determined by a one-way ANOVA for each genotype. Letters indicate the different significance groups after Duncans' pairwise comparisons $(P<0.01)$. Means followed by the same letter are not different at the $P<0.01$ level of significance.

\section{Striga impact on crop biomass and yields}

To estimate the genotypic differences in the extent of damage caused by Striga infection, crop dry shoot biomass and grain yield were analysed. Korokollow still had reasonable biomass and grain yield compared to the other genotypes (Table 3) in spite of high Striga infection (Figure 1). Similarly, biomass and grain yield of genotypes Mogud and Wadbaco 
were also reasonable (Table 3) despite the high Striga infection (Figure 1) and seed yields were even higher than that of the Striga resistant SRN39 (Table 3). In contrast, Wadfahel and Fakimustahi had low biomass and grain yield compared with the other genotypes (Table 3).

\section{Discussion}

In central and eastern Sub-Saharan Africa where sorghum is produced under rainfed conditions, often in continuous monocropping and under low-input farming with inherent poor soil fertility, infestation of the fields by the root parasitic weed Striga has become extremely severe. ${ }^{1,4,47,48}$ Of several factors, such as sowing date and soil fertility, host cultivar appears to be one of the most important factors influencing the degree of damage caused by the parasite. ${ }^{49,50}$ The use of a fully resistant genotype would not only reduce infection but also prevent further build-up of the Striga seed bank. However, a fully resistant cultivar has so far not been identified. ${ }^{45}$ Using a laboratory screening method, Ejeta and coworkers identified various components of resistance, such as low germination stimulation, low haustorial initiation, mechanical barriers and antibiosis. 9,19

Strigolactones are the best described class of Striga germination stimulants, and a lower production of these compounds was shown to result in a decrease in Striga infection in several crop species. ${ }^{51-53}$ Ramaiah and Ejeta and coworkers developed sorghum genotypes with lower levels of germination stimulants, and these genotypes were shown to be more resistant to Striga under field conditions than most of the newly introduced high yielding cultivars. ${ }^{8,21,22,38,54}$

In the present study, the concentrations, composition, and biological activity of strigolactones in sorghum root exudates were examined under controlled conditions in Wageningen, to delineate their relevance to Striga parasitism under field conditions, in Sudan. Strigolactone production by plants has been demonstrated to be strongly modulated by environmental conditions. Low availability of phosphate, for example, strongly enhances secretion of strigolactones, in red clover, tomato, rice and sorghum. ${ }^{43,53,55}$ Indeed, 
in our strigolactone collection procedure, we also applied phosphate starvation to boost strigolactone formation. In the field in Sudan, the availability of phosphate likely differs from the situation under controlled conditions. We expect that this will affect the absolute amount of strigolactone exudation, but not the relative differences between genotypes in strigolactones production and composition. The relation between strigolactone production under controlled conditions and Striga field infection reported in this study suggests that there is a strong functional link between the genetic potential of strigolactone production and Striga infection.

In vitro germination bio-assays showed that the strigolactones, 5-deoxystrigol and sorgomol, present in sorghum root exudates, are potent germination stimulants of the Striga ecotype used in the present study. ${ }^{56}$ These bio-assay results are paralleled by higher infection levels and increased susceptibility of high 5-deoxystrigol and sorgomol producing genotypes under field conditions. In contrast, a lower abundance of these two strigolactones confers a certain level of field resistance. The results of this study showed that Fakimustahi and Wadfahel, genotypes with a high abundance of 5-deoxystrigol and sorgomol in their root exudate (Fig. 3), exhibited high susceptibility to Striga in the field manifested in a greater number of emerged Striga plants and a high Striga biomass (Figure 1 and Table 1). On the other hand, Mogud and Wadbaco, with much less abundance of 5deoxystrigol and sorgomol in their root exudates (Fig. 3), exhibited higher field resistance, which was manifested in the low emergence and biomass of the parasite (Figure 1 and Table 1). It is noteworthy that among all genotypes evaluated in the present study, SRN39, a Striga resistant genotype, produced low amounts of 5-deoxystrigol and sorgomol (Figure 3) and sustained the lowest Striga emergence and biomass (Fig. 1 and Table 1).

Pearson correlation showed that the in vitro stimulation of Striga seeds by root exudates of the different genotypes positively correlates with the abundance of 5-deoxystrigol (Table 2). Moreover, there is a positive relationship between the 5-deoxystrigol and - to a lesser extent - sorgomol concentration in the root exudate and Striga infection in the field (Fig. 5). This finding corroborates previous findings 18,56 on the relationship between germination stimulant activity and Striga infection levels in the field and is in line with a 
report by Jamil et al (2013) ${ }^{53}$ who demonstrated a relationship between strigolactone production by Malian sorghum genotypes in the greenhouse in Wageningen and Striga infection in the field in Mali. Application of diammonium phosphate fertiliser decreased strigolactone production under controlled conditions and reduced Striga infection in the field. ${ }^{53}$

These results are also in agreement with those of Yoneyama and coworkers who compared germination stimulant activity of different sorghum cultivars and demonstrated that the abundance of 5-deoxystrigol in the root exudate of the susceptible Sudanese sorghum cultivar Tabat is 35-fold greater than in the root exudate of the Striga-resistant SRN39. ${ }^{57}$ They concluded that susceptibility of sorghum cultivars to Striga could be related, in part, to the production and/or exudation of 5-deoxystrigol, which they defined as a stable strigolactone. In the present study we also found a negative relationship between susceptibility to the parasite and the amount of orobanchol in the root exudates. Orobanchol has not been reported in sorghum before and the negative relationship between orobanchol and susceptibility of sorghum to Striga is puzzling. However, the possibility of a common precursor for the two strigolactones cannot be ruled out. In such a case high amounts of orobanchol would automatically result in low concentrations of 5-deoxystrigol and therefore in lower infection. Intriguingly, the results show that low germination inducers are not necessarily low strigolactone producers (Fig. 3, 4). Since our study only involved a limited set of genotypes it would be good to expand this investigation to include more cultivars, in order to confirm our findings.

Resistance to Striga has occasionally been described as a mechanism that ensures low infection and high (or satisfactory) host yields. ${ }^{12,38,58}$ While tolerance to Striga infection, the opposite of sensitivity, has been described ${ }^{35}$ as the host's ability to support a certain degree of Striga infection without suffering significant loss in yield and/or product quality. Resistance and tolerance to Striga are often confused due to the absence of complete field resistance to Striga so far. Low amounts of active stimulant e.g. 5-deoxystrigol and sorgomol in Mogud and Wadbaco (Fig. 3), confer pre-attachment resistance and may account, at least in part, for the reduced field susceptibility of these genotypes to Striga. 
However, existence of post-attachment resistance cannot be ruled out at this stage. Wadbaco, Mogud and particularly Korokollow produced satisfactory grain yield and crop biomass in spite of Striga infection in both locations (Table 3; Fig. 1). This finding may provide evidence for the presence of tolerance to infection by the parasite in Korokollow and, to a lesser extent, in Wadbaco and Mogud. Our results are in accordance with earlier statements that under environments of traditional farming systems where Striga seed is always present in the soil most local varieties display tolerance, sustaining a reasonable yield in spite of Striga infestation, as well as a certain degree of resistance. ${ }^{27,34,59}$ The observed field resistance in Mogud, in the present study, confirms earlier work in Sudan by Wilson-Jones (1953) ${ }^{60}$ and Khidir (1983) ${ }^{61}$ describing Mogud as relatively resistant to Striga and agrees with farmers' perception about Wadbaco as less affected by Striga infection. The lack of field resistance in Wadfahel and Fakimustahi to Striga infection is consistent with a lack of pre-attachment resistance attributable, at least in part, to the high germination inducing activity of the root exudate associated with high amounts of 5deoxystrigol and sorgomol.

\section{Conclusions}

The strigolactone profile of the root exudates of the sorghum genotypes produced under controlled conditions seemed to be associated with the resistance and susceptibility of the same genotypes under field conditions. Varieties with high 5deoxystrigol levels in root exudates, had higher Striga infection in the field, while those producing high orobanchol concentrations had low infection levels. Identification of the underlying genetic region and/or mechanism for the strigolactone-based resistance, identified in the present study, would potentially allow the transfer of this Striga resistance to susceptible sorghum genotypes, such as was also achieved for the low germination stimulant allele identified by Ejeta and co-workers. The use of this trait in combination with post-attachment resistance mechanisms would improve the durability of the resistance to the parasite. The 
occurrence of Striga tolerance in some local Sudanese sorghum genotypes may provide the starting material for the transfer of tolerance QTLs and/or genes to elite cultivars.

\section{Acknowledgements}

The authors acknowledge funding by NUFFIC (to NMA) and Wageningen UR (WUR sandwich fellowship to NMA) and the Netherlands Organization for Scientific Research (to HJB VICI Grant, 865.06.002 and Equipment grant, 834.08.001). Kind support during execution of the field trials was provided by The Japan International Cooperation Agency (JICA) project on improvement of food security in semi-arid regions of the Sudan through management of root parasitic weeds implemented by the College of Agricultural Studies, Sudan University of Science and Technology (SUST) Khartoum, Sudan. The authors also thank Dr Ayman Awad and co-workers at the Agricultural Research Corporation (ARC), Sudan, Gadarif Research Station for technical assistance. We would like to thank Prof. Binne Zwanenburg, Prof. Koichi Yoneyama and Prof. Tadao Asami for providing SL standards. 


\section{References}

1. Ejeta G, The Striga scourge in Africa: a growing pandemic, in Integrating new technologies for Striga control: towards ending the witch-hunt, ed. Ejeta G, Gressel J. World Scientific, Singapore, pp. 3-16 (2007).

2. Gressel J, Hanafi A, Head G, Marasas W, Obilana AB, Ochanda J, Souissi T and Tzotzos G, Major heretofore intractable biotic constraints to African food security that may be amenable to novel biotechnological solutions. Crop Prot 23(8):661-689 (2004).

3. Labrada, R. Farmer training on parasitic weed management. In: Progress on Farmer Training in Parasitic Weed Management, (Labrada, R., ed.), FAO, Rome, pp.1-5 (2008).

4. Scholes JD and Press MC, Striga infestation of cereal crops - an unsolved problem in resource limited agriculture. Curr. Opin. Plant Biol. 11(2): 180-186 (2008).

5. Parker $\mathrm{C}$ and Riches CR. Parasitic Weeds of the World: Biology and Control. CAB International, Wallingford, UK, pp. 111-163(1993).

6. Spallek T, Mutuku M and Shirasu K. The genus Striga: a witch profile. In Mol Plant Pathol, Wiley Online Library, pp. 861-869 (2013).

7. Bouwmeester, H.J., Matusova, R., Zhongkui, S. \& Beale, M.H. Secondary metabolite signaling in hostparasitic plant interactions. Curr. Opin. Plant Biol. 6, 358-364 (2003).

8. Ejeta G Breeding for Striga resistance in sorghum: Exploitation of an intricate host- parasite biology. Crop Sci. 47, S216-S227(2007).

9. Yoder, J. I. \& Scholes, J. D. Host plant resistance to parasitic weeds; recent progress and bottlenecks. Curr. Opin. Plant Biol. 13, 478-484 (2010).

10. Timko, M. P. and J. D. Scholes. Host Reaction to Attack by Root Parasitic Plants. Parasitic Orobanchaceae. D. M. Joel, J. Gressel and L. J. Musselman, Springer, Berlin Heidelberg, pp. 115141(2013).

11. Hassan, M.M., M.E. Gani, and A.G. Babiker. Management of Striga hermonthica in sorghum using soil rhizosphere bacteria and host plant resistance. Int. J. Agric. Biol. 11:367-373 (2009).

12. Hess DE, Haussmann BIG, Status quo of Striga control:prevention, mechanical and biological control methods and host plant resistance. In: Kroschel J, Mercer-Quarshie H, Sauerborn J (eds) Advances in parasitic weed control at on-farm level.Joint action to control Striga in Africa, vol 1. Margraf Verlag, Weikersheim, pp 75-87(1999).

13. Kim, S. K. (ed). Combating Striga in Africa. Proceedings, International Workshop organized by IITA, ICRISAT, and IDRC, 22-24 August 1988. IITA, Ibadan, Nigeria, pp. 151 (1991).

14. Sauerborn J. Parasitic Flowering Plants : Ecology and Management, ed. Josef Margraf, Weikersheim, Germany (1991).

15. Babiker, A.G.T., Striga control in Sudan: An integrated approach. In: Sorghum and Pearl Millet Diseases, Leslie, J.F. (Ed.), Iowa State Press, pp. 159- 163(2002).

16. Ejeta G. Integrating biotechnology, breeding, and agronomy in the control of the parasitic weed Striga spp in sorghum. In In the Wake of the Double Helix: From the Green Revolution to the Gene Revolution, Tuberosa R, Phillips RL, Gale M (Eds) Bologna, Italy, pp. 239-251 (2005).

17. Tesso TT and Ejeta G, Integrating Multiple Control Options Enhances Management and Sorghum Yield on Heavily Infested Soils. Agron J 103(5):1464-1471 (2011).

18. Haussmann BIG, Hess DE, Welz HG, Geiger HH.Improved methodologies for breeding striga-resistant sorghums (review article). Field Crops Res 66(3):195-201(2000). 
19. Ejeta G, Rich PJ and Mohamed A. Dissecting a complex trait to simpler components for effective breeding of sorghum with a high level of Striga resistance, in Integrating new technologies for Striga control towards ending the witch-hunt, ed. by Ejeta G, Gressel J, World Scientific, Singapore, pp. 87-98 (2007).

20. Mohamed A, Ellicott A, Housley TL and Ejeta G, Hypersensitive Response to Striga Infection in Sorghum. Crop Sci. 43(4): 1320-1324 (2003).

21. Haussmann B, Hess D, Omanya G, Reddy B, Welz H and Geiger H, Major and minor genes for stimulation of seed germination in sorghum, and interaction with different Striga populations. Crop Sci. 41(5): 15071512 (2001).

22. Hess DE, Ejeta G and Butler LG, Selecting sorghum genotypes expressing a quantitative biosynthetic trait that confers resistance to Striga. Phytochem 31(2): 493-497 (1992).

23. Ejeta G, Mohamed A, Rich P, Melake-Berhan A, Housley TL, Hess DE. Selection for specific Striga resistance mechanisms in sorghum. In: BI G Haussmann, DE Hess, M, L Koyama, L. Grivet, H,F,W. Ratunde and H.H Geiger (eds). Breeding for Striga resistance in cereals. Proc. Of a Workshop held at IITA, Ibadan, Nigeria, from 18-20 August. p. 18-20 (1999).

23. Ejeta G, Mohamed A, Rich P, Melake-Berhan A, Housley T and Hess D. Selection for specific Striga resistance mechanisms in sorghum. In BIG Haussmann, DE Hess, M, L Koyama, L Grivet, H, F, W Ratunde and HH Geiger (eds) Breeding for Striga resistance in cereals Proc Of a Workshop held at IITA, Ibadan, Nigeria, from, pp. 18-20 (1999).

24. Haussmann BIG, Hess DE, Omanya GO, Folkertsma RT, Reddy BVS, Kayentao M, Welz HG and Geiger $\mathrm{HH}$, Genomic regions influencing resistance to the parasitic weed Striga hermonthica in two recombinant inbred populations of sorghum. Theor Appl Genet 109(5): 1005-1016 (2004).

25. Bantilan MCS, Gowda CLL, Reddy BVS, Obilana AB and Evenson RE. Sorghum Genetic Enhancement: Research Process, Dissemination and Impacts. International Crops Research Institute for the Semi-Arid Tropics, Patancheru, Andhra Pradesh, India, (2004).

26. Tefera H, Reston VA, Dahlberg J and Smale M, External Evaluation Report on the Sorghum, Millet and Other Grains (SMOG)/INTSORMIL Collaborative Research Support Program (CRSP) (2012).

27. Gurney AL, Press MC and Scholes JD, Can wild relatives of sorghum provide new sources of resistance or tolerance against Striga species? Weed Res 42(4): 317-324 (2002).

28. Haussmann, B.I.G., Hess, D.E., Koyama, M.L., Grivet, L., Rattunde, H.F.W., Geiger, H.H. (Eds.), Breeding for Striga Resistance in Cereals. Margraf Verlag, Weikersheim. (2000).

29. Satish K, Gutema Z, Grenier C, Rich PJ and Ejeta G, Molecular tagging and validation of microsatellite markers linked to the low germination stimulant gene (lgs) for Striga resistance in sorghum [Sorghum bicolor (L.) Moench]. Theor Appl Genet 124(6): 989-1003 (2012).

30. Haussmann BIG, Hess DE, Omanya GO, Folkertsma RT, Reddy BVS, Kayentao M, Welz HG and Geiger $\mathrm{HH}$, Genomic regions influencing resistance to the parasitic weed Striga hermonthica in two recombinant inbred populations of sorghum. Theor Appl Genet 109(5): 1005-1016 (2004).

31. Grenier C, Ibrahim Y, Haussmann BIG, Kiambi D and Ejeta G, Marker-assisted selection for Striga resistance in sorghum, in Integrating New Technologies for Striga Control, Towards Ending the Witchhunt, ed. by Ejeta G, Gressel J, World Scientific, Singapore, 159-172 (2007).

32. Mohamed A, Ali R, Elhassan O, Suliman E, Mugoya C, Masiga CW, Elhusien A and Hash CT, First products of DNA marker-assisted selection in sorghum released for cultivation by farmers in sub-saharan Africa. Mol Plant Breed 3(1): 3 (2014).

33. Haussmann BIG, Hess DE, Welz HG and Geiger HH, Improved methodologies for breeding Strigaresistant sorghums. Field Crops Res 66(3): 195-211 (2000).

34. Rodenburg J, Bastiaans L, Weltzien E and Hess DE, How can field selection for Striga resistance and tolerance in sorghum be improved? Field Crops Res 93(1): 34-50 (2005). 
35. Rodenburg J and Bastiaans L, Host-plant defence against Striga spp.: reconsidering the role of tolerance. Weed Res 51(5): 438-441 (2011).

36. Rodenburg J, Bastiaans L and Kropff MJ, Characterization of host tolerance to Striga hermonthica. Euphytica 147(3): 353-365 (2006).

37. Van Ast A, Bastiaans L and Kropff MJ, A comparative study on Striga hermonthica interaction with a sensitive and a tolerant sorghum cultivar. Weed Research 40(6): 479-493 (2000).

38. Rodenburg J. The role of sorghum genotype in the interaction with the parasitic weed Striga hermonthica. $\mathrm{PhD}$ thesis, Wageningen University, Wageningen, The Netherlands (2005).

39. Bantilan, M. C. S., Deb, U. K., Gowda, C. L. L., Reddy, B. V. S., Obilana, A. B., and Evenson, R. E., in Sorghum Genetic Enhancement: Research Process, Dissemination and Impacts. International Crops Research Institute for the Semi-Arid Tropics, Patancheru, Andhra Pradesh, India, pp. 109 (2004).

40. Kapran I, Grenier C and Ejeta G. Introgression of genes for Striga resistance into African landraces of sorghum, in Integrating New Technologies for Striga Control: Towards Ending the Witch-Hunt, World Scientific, Singapore, pp. 129-141 (2007).

41. Jamil M. The relationship between strigolactones and Striga hermonthica infection in cereals. PhD thesis, Wageningen University, Wageningen, The Netherlands (2012).

42. Babiker A and Reda F. Recent advances in Striga research in Sudan and Ethiopia. In 5 International Symposium of Parasitic Weeds, CIMMYT, Nairobi (Kenya), 24-30 Jun 1991. (1991).

43. Lopez-Raez JA and Bouwmeester H, Fine-tuning regulation of strigolactone biosynthesis under phosphate starvation. Plant Signal Behav 3(11): 963-965 (2008).

44. Zhang Y, Dijk vADJ, Scaffidi A, Flematti GR, Hofmann M, Charnikhova T, Verstappen FWA, Hepworth J, Krol vdAR and Leyser O, Rice cytochrome P450 MAX1 homologs catalyze distinct steps in strigolactone biosynthesis. Nat Chem Biol 10: 1028-1033 (2014).

45. Arnaud M-C, Véronési C and Thalouarn P, Physiology and histology of resistance to Striga hermontica in Sorghum bicolor var. Framida. Aust. J. Plant Physiol 26: 63-70 (1999).

46. Matusova R, van Mourik $\mathrm{T}$ and Bouwmeester HJ, Changes in the sensitivity of parasitic weed seeds to germination stimulants. Seed Sci. Res. 14(04): 335-344 (2004).

47. Babiker, A.G.T., Striga: The spreading scourge in Africa. JSCRP 42: $74-87$ (2007).

48. Parker C, Observations on the current status of Orobanche and Striga problems worldwide. Pest Manag Sci 65(5): 453-459 (2009).

49. Mutengwa CS, Tongoona PB and Sithole-Niang I, Genetic studies and a search for molecular markers that are linked to Striga asiatica resistance in sorghum. Afr. J. Biotechnol 4(12) (2005).

50. Noubissie, J. B. T., Yadji, H. T., \& Baldena, I. Screening Sorghum Populations for Resistance to Striga hermonthica (Del.) Benth. in Northern Cameroon. Ann Biol Res 3, 2357-2364 (2012).

51. Jamil M, Charnikhova T, Cardoso C, Jamil T, Ueno K, Verstappen F, Asami T and Bouwmeester HJ, Quantification of the relationship between strigolactones and Striga hermonthica infection in rice under varying levels of nitrogen and phosphorus. Weed Research 51(4): 373-385 (2011).

52. Jamil M, Rodenburg J, Charnikhova T and Bouwmeester HJ, Pre-attachment Striga hermonthica resistance of New Rice for Africa (NERICA) cultivars based on low strigolactone production. New Phytol 192(4): 964-975 (2011).

53. Jamil M, Van Mourik TA, Charnikhova $\mathrm{T}$ and Bouwmeester HJ, Effect of diammonium phosphate application on strigolactone production and Striga hermonthica infection in three sorghum cultivars. Weed Research 53(2): 121-130 (2013).

54. Haussmann BIG, Hess DE, Reddy BVS, Mukuru SZ, Kayentao M, Welz HG and Geiger HH, Quantitativegenetic parameters of sorghum growth under striga infestation in Mali and Kenya. Plant Breed 120(1): 49$56(2001)$. 
55. Yoneyama K, Yoneyama K, Takeuchi Y and Sekimoto H, Phosphorus deficiency in red clover promotes exudation of orobanchol, the signal for mycorrhizal symbionts and germination stimulant for root parasites. Planta; 225(4): 1031-1038 (2007).

56. Nasreldin MA, T. C., Tatsiana Charnikhova, Emilie Fradin, Juriaan Rienstra, A.G.T. Babiker and Harro J. Bouwmeester. Characterization of the genetic variation in resistance to witchweed (Striga hermonthica) in sorghum (Sorghum bicolor) germplasm from Sudan, in prepration.

57. Yoneyama K, Awad AA, Xie X, Yoneyama K and Takeuchi Y, Strigolactones as germination stimulants for root parasitic plants. Plant Cell Physiol 51(7): 1095-1103 (2010).

58. Ejeta, G., Butler, L.G., Hess, D.E., Vogler, R.K., Genetic and breeding strategies for Striga resistance in sorghum. In: Ransom, J.K., Musselman, L.J., Worsham, A.D., Parker, C. (Eds.), Proceedings of the Fifth International Symposium of Parasitic Weeds. CIMMYT, Nairobi, Kenya, pp. 539-544 (1991).

59. Rao MJV. Patterns of resistance to Striga asiatica in sorghum and millets, with special reference to Asia. In ICRISAT Confernce No 196. ICSU and International Development Research Center, Dakar,Senegal, pp. 71-92 (1983).

60. Wilson Jones K, The witchweeds of Africa. World Crops 5(7): 263-266 (1953).

61. Khidir MO. Striga Research in the Sudan. In Second Regional workshop on Sorghum and Millet Improvement in Eastern Africa, Ruanda (1983). 


\section{Chapter 6}

\section{General Discussion}

In this thesis I have investigated strigolactone production in 36 sorghum accessions and in a number of inter- and rotation crops (sesame, groundnut and millet). Strigolactones, or germination stimulants, form the core element of low stimulant resistance and suicidal germination and with my work - that was done in the lab, greenhouse and field - I hope to contribute to a better understanding of the roles of strigolactones in Striga resistance, susceptibility and control (Chapter 2, 4 and 5), as well as their role in tolerance to Striga in sorghum (Chapter 3). Here, in Chapter 6, I discuss the results in my thesis in a broader perspective.

As explained in the General Introduction Striga spp. cause enormous damage worldwide to major food crops including sorghum, maize, rice, pearl millet and sugar cane (Babiker,2007; Gressel et al., 2004). Many different control options have been explored for Striga and in my thesis I focus on control mechanisms that involve strigolactones. Strigolactones induce the first step in the Striga life cycle: germination and may play a role in later steps of the lifecycle as well. Options for control based on germination stimulants have been suggested before, for example, by exploring low germination resistance in sorghum, as well as the use of suicidal germination to reduce the Striga seedbank. As germination of Striga depends entirely upon exudation in the rhizosphere of the active stimulants (strigolactones), exudation of less active strigolactones has been described as an important resistance trait in sorghum (Ejeta, 2007). However, such resistant varieties are often challenged by the presence of a massive seed bank accumulated over the years. In that case, non-hosts, trapcrops and intercrops that induce suicidal germination can help to reduce the Striga seed bank (Sun et al., 2010). Finally, some host genotypes have been shown to exhibit some degree of tolerance to Striga infection which helps to guarantee some level of yield even if Striga infection cannot be prevented (Bebawi; 1991; Gbehounou 
et al., 2003; Botanga et al., 2003; Traore et al., 2011). In my thesis I wanted to better investigate the tolerance mechanism and the possible involvement of strigolactones in this. What is known about role and type of strigolactones in sorghum, and intercrops?

Sorghum and other Striga host and non-host plants release naturally in the rhizosphere a mixture of strigolactones varying in structures, amounts, and extent of Striga stimulation activity (Awad et al., 2006; Lopez-Raez et al., 2008; Xie et al., 2010). Except in rice, strigolactones identified in most grasses are strigol-like (Awad et al., 2006; Xie et al., 2013). Hauck et al., (1992) and Awad et al., (2006) documented exudation of 5deoxystrigol, sorgomol, strigol and sorgolactone, all strigol-type strigolactones, as the major germination stimulants in sorghum. In this thesis in some of the sorghum genotypes I detected exudation of orobanchol-type strigolactones including orobanchol and ent-2 'epi-5-deoxystrigol as well as variable amounts of strigol-type stimulants including 5deoxystrigol and sorgomol, and in addition in some sorghum cultivars, low concentrations of strigol and sorgolactone. Production of orobanchol-type strigolactones had never been reported in sorghum until recently, possibly due to difference in exudates collection, concentration and purification. 5-Deoxystrigol, strigol and sorgomol were earlier detected in some sorghum, maize and pearl millet cultivars (Siame et al., 1993; Awad et al., 2006; Xie et al 2008a; Yoneyama et al., 2009; Charnikhova et al., 2017) but orobanchol had been described only in rice, red clover and tomato (Akiyama and Hayashi 2006). In this thesis I detected exudation by two pearl millet cultivars from Sudan of high amounts of orobanchyl acetate and low concentrations of 5-deoxystrigol, orobanchol, sorgomol and a sorgomollike strigolactone (Chapter 2). In pearl millet, Awad et al., (2006) and Jamil et al., (2014) showed production of 5-deoxystrigol, orobanchol, and orobanchyl acetate. Orobanchyl acetate had been characterized only in cowpea, red clover and soybean (Xie et al. 2008b; Ueno et al., 2011). However, the strigolactone profile of several other Striga non-host crops often used to induce suicidal germination and suppress Striga infestation e.g. sesame and groundnut had not been identified so far. In my thesis work in sesame root exudates I detected 5-deoxystrigol and orobanchol, and sorgolactone-like, solanocol-like and didehydro-orobanchol isomer-like strigolactones while in groundnut I detected high 
amounts of 5-deoxystrigol and low amounts of orobanchol, and sorgolactone-like and didehydro-orobanchol isomer-like strigolactones (Chapter 4). The results show that there is strong biosynthetic variation in strigolactone type and concentration between crop species as well as among cultivars of the same plant species. Similar species/varietal variation in strigolactone exudation has been reported in Striga host and non-host crop species e.g. rice, maize and tomatoes (Jamil et al., 2011; Xie et al., 2013; Kohlen et al., 2013; Lopez-Raez et al., 2008). Structural variation in the strigolactones produced by plants has been suggested to influence susceptibility to Striga in the field (Yoneyama et al., 2015). Structure/activity studies showed that strigol-type strigolactones, e.g. 5deoxystrigol, sorgomol, strigol and sorgolactone, are much more effective in stimulating Striga seeds germination than orobanchol-type e.g. orobanchol and orobanchyl actetate (Akiyama et al., 2010; Nomura, et al., 2013; Cardoso et al, 2014; Sugimoto et al., 1998; Yoneyama et al., 2009). In Chapter 2 using authentic strigolactone standards I showed also that orobanchol induces poor Striga seed germination $(<10 \%$ germination), whereas 5 deoxystrigol, sorgomol, sorgolactone and strigol induce much higher germination $>50 \%$. Studies have shown that exudation of low/less active Striga germination stimulants (strigolactones) in sorghum is associated with field resistance to Striga (Gobena et al., 2017) and in pearl millet it has also been hypothesized that the difference in the nature of the germination stimulant produced is behind the observed lower Striga infection when pearl millet is cultivated on Striga infested sorghum fields (Ramaiah, 1987, Hess et al., 1992; Vasudeva Rao, 1985; Rich et al., 2004; Mohemed et al., 2016). This will be discussed further below. Striga resistant genotypes are defined as those that support few or no Striga attachments when grown in an infested soil (Doggett, 1988; Ejeta et al., 1992; Parker and Riches, 1993). Many authors have attempted to identify Striga resistant cultivars and their reactions to Striga infection. Based on stimulant activity some sorghum cultivars such as SRN39, IS-9830, Hakika, Tetron and Framida cultivars have been identified as low stimulant genotypes and consequently selected and released for cultivation in Striga endemic areas (Rich et al., 2004; Ejeta et al., 2007; Kambal, 1979; Bebawi, 1981, Ejeta, 2007). Although later the Low germination stimulant (lgs) gene and 
associated molecular marker associated with Striga resistance had been found in a population of recombinant inbred lines (RILs) derived from SRN39 and the susceptible cultivar Shanqui Red (SQR), the biochemical nature of the resistances was not fully understood (Ejeta, 2007; Satish et al., 2012). Striglactones profiling showed that SRN39 and Striga-susceptible sorghum cultivars exuded the same strigolactone structures e.g. 5deoxystrigol and sorgomol but in different amounts (Yoneyama et al., 2010). Lately, Gobena, et al., (2017) showed that expression of the LGS gene in SRN39 is low and associated with loss of function which affects stimulant stereochemistry leading to production of a high orobanchol/low5-deoxystrigol profile which results in decreased seed germination. In contrast, expression of $L G S$, in the susceptible cultivar; Shanqui-Red is present and this results in production of a high 5-deoxystrigol/low orobanchol profile which is associated with high germination activity and high field Striga infection (Gobena, et al., 2017). In this thesis, based on strigolactones profiling, root exudate germination bioassays and genetic marker analysis I showed that resistance to Striga in sorghum cultivars IS-9830, Hakika, Tetron and Framida is due to low expression of the same $L G S$ gene. I showed that root exudates of these cultivars produce similar high orobanchol/low 5-deoxystrigol strigolactone profiles that trigger low Striga seed germination. In addition, the altered strigolactone profile also resulted in a delayed time of Striga emergence and reduced Striga emergence per pot. Genotyping these cultivars showed the presence of the LGS1 SRN39 genetic marker. In Chapter 2 and 5 I showed that production of a high 5deoxystrigol/low orobanchol profile results in high susceptibility to Striga - with early Striga emergence and higher infection - in the field in genotypes such as Fakimustahi and Wadfahel (Chapter 5) and in in pot experiments in Gadam Elhamam, Ferterita Geshaish, Naten, Najad and Hariri (Chapter 2). This is associated with higher root exudate germination stimulatory activity and a lack of the LGS1 marker. In contrast, the presence of high orobanchol/low 5-deoxystrigol and sorgomol in Wadbaco and Mogud sorghum genotypes resulted in delayed Striga emergence and overall reduction of Striga infection in the field and in pot experiments and this was associated with a reduction in the root exudate Striga germination stimulatory activity. This was associated with the absence of 
the LGS1 SRN39 genetic marker and the presence of the LGS1 Shanqui-Red marker. The PCA bi-plot (Fig 7A, Chapter 2) showed that Striga resistant genotypes such as in SRN39, Tetron, Framida, Hakika, Mogud and Wadbaco were clearly associated with production of orobanchol and conversely susceptible genotypes such as Feterita Geshaish, Tabat, Wadfahel, Fakimustahi, Bari and Dari were associated with high production of 5deoxystrigol and sorgomol. I concluded that in the absence of other Striga resistance mechanisms - a high 5-deoxystrigol and/or sorgomol/low orobanchol strigolactone profile in the root exudate of sorghum increases susceptibility to Striga while high orobanchol/low 5-deoxystrigol and/or sorgomol strigolactone profile increases resistances. The results confirm findings of (Gobena, et al., 2017) in sorghum that low expression of LGS1 resulted in production of a high orobanchol/low 5-deoxystrigol profile which conferred low stimulant resistance to Striga. Interestingly, in a similar fashion, I showed that a high orobanchyl acetate/low 5-deoxystrigol profile in the root exudate of pearl millet results in reduced germination stimulatory activity and reduced infection by the sorghum Striga ecotype of pearl millet (Chapter 4). It seems therefore that a high orobanchol-type vs low strigol-type strigolactone composition in the root exudate of cereals causes resistance at least against the sorghum ecotype of Striga.

Studies on the effect of intercropping on Striga control have shown that effectiveness of intercrop genotypes such as soybean and cowpea to induce Striga germination in the laboratory was highly correlated with their effectiveness in reducing parasitism on susceptible sorghum in the field (Benson, 1982). Thus, the production by intercrops of highly active stimulants has been hypothesized to trigger efficient suicidal germination (Sun et al., 2010; Traore et al., 2011). I show in this study that high 5-deoxystrigol and sorgomol-like /low orobanchol strigolactone profiles in the root exudates of inter-crops sesame and groundnut resulted in higher stimulatory activity and reduced Striga emergence on the intercropped sorghum likely due to suicidal germination (Chapter 4). Correlation analysis showed that the numbers of emerged Striga plants on sorghum was inversely correlated with Striga seed germination induced by intercrop root exudates, suggesting occurrence of suicidal germination. The 5-deoxystrigol concentration in the root exudate 
of the intercrops also showed a positive, though non-significant, correlation with Striga seed germination, but negatively and significantly correlated with the emerged Striga plants per pot. In the regression analysis 5-deoxystrigol in the root exudate of the intercrops was the only significant, negative, predictor to Striga emergence on the intercropped sorghum suggesting that the compound is responsible for suicidal germination. However, a contribution of other only tentatively identified strigolactones such as solanacol-like and didehydro-orobanchol isomer-like in these inter-crops to a reduction in Striga parasitism through suicidal germination or post-germination interference - cannot be disregarded. It has been suggested that semiochemicals in the inter-crop root exudates may perturb temporal and/or spatial growth of the parasite radicle thus reducing attachments to the host roots and consequently parasitism (Khan et al., (2002). The mutual effects of germination stimulants and post germination inhibitors has been considered as novel and highly efficient mechanism of suicidal germination of Striga seeds (Khan et al., 2008). Synergetic interactions between strigolactones and the microbial communities present in the rihzosphere together possibly also may affect the magnitude of Striga seed germination and final emergence (Xie et al., 2010; Schlemper et al., 2017). From a breeding perspective it is relevant to find out how each of the strigolactones detected correlates with Striga germination and parasitism, as an indication for their significance. Identification of the unknown strigolactones in the exudates of sesame and groundnut would be an important first step towards the elucidation of their effect, on germination as well as on postgermination processes and the microbiome.

Vasudeva Rao, (1985) reported that the simple correlation coefficients between stimulant production and Striga numbers obtained from different trials were positive and at some locations and trials are very high indicating that stimulant production could be a useful indicator of field reaction. However, so far there were no report describing correlations between the concentration of specific germination stimulants $=$ strigolactones and Striga resistance traits in sorghum. In this thesis for the first time correlation analyses between specific germination stimulants and Striga infection traits were conducted and this showed that different strigolactones exhibited different associations with Striga seed germination 
and possibly other resistances forms and tolerance. A high concentration of specific strigolactones is predictive of the final Striga infection level and also gives an indication about host tolerance to Striga. In Chapter 2, 3 and 5 I showed that orobanchol concentration negatively correlated with Striga germination and Striga emergence but positively correlated with host tolerance to Striga. In contrast, the concentration of 5-deoxystrigol positively correlated with Striga germination and Striga emergence but negatively with host tolerance to Striga. I showed that the two compounds were negatively associated to each other suggesting a negative biosynthetic correlation between the two strigolactones. In Chapter 2 results of stepwise regression showed that orobanchol is the best negative predictor to Striga emergence and 5-deoxystrigol the best positive predictor. These results provide strong support for findings of Gobena et al., (2017) that high orobanchol/low 5deoxystrigol production would decreases susceptibility and results in increased resistance to Striga, but once more here in this thesis

I found orobanchol also increases tolerance to Striga and reduces parasitism efficiency. Indeed, I found a good correlation between root exudate induced in vitro Striga germination and Striga infection in pot and field experiments for majority of genotypes, with a few exceptions of genotypes that showed inconsistency between germination in the lab and performance in the pot experiment, likely due to variation in other, post-germination, resistance mechanisms.

The results in particular suggest that the highly virulent Striga ecotype, "Abu Naama" collected from under sorghum from Sudan is less sensitive to orobanchol and orobanchyl acetate germination stimulants but highly sensitive to the germination stimulants 5deoxystrigol, sorgomol, sorgolactone and strigol. This suggests that selection for high orobanchol/low 5-deoxystrigol, sorgomol, sorgolactone and strigol profiles could be an effective strategy to obtain resistant genotypes that moreover will also display tolerance to Striga. A better understanding of the mechanism that underlies this difference in sensitivity - probably related to different strigolactone receptors in this ecotype - is important to prevent that this resistance will be lost due to adaptation of Striga. Combination of this form of resistance with others would also be important to prevent this from happening. 
Even though LGS and other, post-attachment, resistances can reduce the parasite load on the host roots even just a few parasite attachments can induce strong negative effects on plant growth and yield in certain sorghum genotypes (Gurney et al., 1999). Here arises the importance of the host's ability to tolerate the negative effects of Striga infection. Tolerance to Striga is defined as the capacity of the host genotype to endure/mitigate the negative impact of infection, to show fewer disease responses and produce a relatively acceptable yield (Van Ast, 2000). The non-linear relation between Striga infection level and yield loss (Gurney et al. (1999) often complicates precise quantification of host tolerance. To improve our understanding of Striga tolerance in this thesis in Chapter3 I investigated the host morphological and physiological responses to Striga infection and I examined the relationship with strigolactone production. Hereto, I investigated whether the presence of specific strigolactones in the root exudate shows a relationship with host tolerance and reduces parasite infection efficiency. In chapter 3 I show that irrespective of Striga infection level the inflicted reduction in biomass and photosynthesis varied significantly between the different genotypes suggesting genotype-specific differences in tolerance to the parasite. Previously in sorghum several authors reported a decline in maximum rate of photosynthesis (A $\max$ ) due to Striga infection as I found also, however, no fall in carboxylation efficiency ( $\Phi \mathrm{CO} 2)$ so far has been documented in sorghum due to Striga infection (Ramlan and Graves, 1996). I suggested in this study particularly in sensitive genotypes that the decreased in $\Phi \mathrm{CO} 2$, which is reflected in a higher $\mathrm{CO}_{2}$ concentration in the sub-stomatal cavities, and reduced stomatal conductance, possibly due to increased $\mathrm{ABA}$, are responsible, at least in part, for the significant reduction in photosynthetic capacity and consequently in the sharp reduction in biomass accumulation. In tolerant genotypes such as Wad Ahmed and Hariri I showed that carboxylation efficiency was less affected by the accumulation of $\mathrm{CO}_{2}$ in the sub-stomatal cavities possibly because the stomata of these genotypes are less affected by changes in ABA concentration. I showed that the tolerant genotypes such as Hariri, Mogud, Wad Ahmed and Wad Baco delayed Striga emergences time even under high Striga infestation, exhibited low to moderate reduction in total biomass, maximum $\mathrm{CO}_{2}$ assimilation rate and 
photosynthetic carboxylation efficiency in comparison to the corresponding Striga free control. In rice Rodenburg et al., (2017) reported that some cultivars such as for example NERICA-10 and sorghum genotype N13, though they have a good resistance, are highly sensitive to Striga even if only one or two parasites are attached. Therefore, in order to control Striga and maintain high yields, both tolerance and resistance are required in cultivars recommended to farmers. In this study I investigated whether the known Striga resistant genotypes SRN39, IS9830 and Tetron possess a certain degree of tolerance. Our study showed that SRN39, IS9830 and Tetron delayed Striga emergence reduced emerged Striga numbers and show relatively low reduction in biomass, $\mathrm{CO}_{2}$ assimilation rate, and photosynthetic carboxylation efficiency compared with non-tolerant genotypes, suggesting that unlike in rice and other sorghum cultivars they combine both resistance and tolerance.

\section{Implications for breeding}

Striga resistance and tolerance are complex traits controlled by the interaction of many genes probably involving several physiological and morphological mechanisms. Identification of genetic sources with effective resistance and tolerance in sorghum germplasm is critical to allow for marker-assisted and conventional breeding for these traits. In field screening it is difficult to uncover resistance and tolerance independently, let alone the underlying mechanisms or gene/genes involved. However, using experiments under controlled conditions as in my Chapter 3 allows to show the presence of genetic variation and genotype-specific tolerance to Striga among sorghum cultivars investigated, highlighting the need for further molecular analysis to elucidate the underlying genomic region involved. In Chapter 3 I showed that Striga infection variably affected maximum photosynthesis, carboxylation efficiency and the $\mathrm{CO}_{2}$ concentration in the sub-stomatal cavity. Further correlation analysis indicated that high orobanchol/low 5-deoxystrigol strigolactone profile positively correlated with host tolerance and in contrast high 5deoxystrigol and sorgomol /low orobanchol negatively associated with tolerance. This suggests that variation in the strigolactone profile and/or carboxylation efficiency could be used as tools for discrimination between Striga tolerant and sensitive genotypes. In Chapter 
3 I showed that SRN39, Tetron and IS9830 combine a significant level of both resistance and tolerance to Striga. Kaewchumnong et al., (2008) in rice showed that QTLs for Striga infection (number, mass and emergence rate) and host plant traits co-located at the same locus suggesting such locus control both Striga establishment (resistance) and its effect of Striga on rice plants (tolerance). To date, few studies have been conducted to map Striga resistance/tolerance QTLs in sorghum. Haussmann et al. (2001, 2004) identified 5 common field resistance QTLs in sorghum from the resistant sorghum cultivars N13 (which shows post-attachment resistance) and IS9830 (a low germination stimulant producer) as a donor parent in the genetic background of a susceptible sorghum cultivar E-36. However, gene/genes underlying those QTLs still need to be identified, cloned and functionally validated. In this thesis IS9830 but not N13 exhibited significant resistance and tolerance to the Striga ecotype used. The modest level of resistance and tolerance displayed by N13 is remarkable and deserves more research to clarify whether this is due to the Striga ecotype used. As mentioned above the low stimulant (QTL) responsible for field resistance in SRN39 was defined as LGS1 which encodes a sulfotransferases. Although the specific function of the sulfotransferase is so far unknown, deletion of LGS1 in SRN39 and other resistant genotypes resulted in a change in the strigolactone profile towards high orobanchol/low 5-deoxystrigol which causes low stimulant activity. Expression of this gene is several folds higher in roots of susceptible Shanqui Red, carrying the wild type (WT) allele LGS1. In this thesis in resistant genotypes Tetron and IS9830, Framida and Hakika I showed the presence of the SRN39 low germination stimulant (LGS1) linked marker, which coincides with relatively high production of orobanchol in the root exudates (Chapter 2) suggesting similar genetics as described before for the resistant sorghum variety SRN39. However, in other genotypes such as Wadfahel, Mogud and Wad Baco, the SRN39 low germination stimulant (LGS1) linked marker was not amplified although root exudates of those genotypes induced low germination and show relatively high orobanchol strigolactone profile (Chapter 2Figs. 4, 7 6B). Instead, amplification resulted in a fragment pattern similar to that of Shanqui Red the high stimulant Striga susceptible Chinese sorghum genotype, suggesting that these genotypes lack the resistance allele at the 
(LGS1) locus. On the other hand, genotypes Tabat and Fakimustahi that produce high levels of 5-deoxystrigol did not show the expected Shanqui Red pattern (Fig. 9C). This may be caused by the possession of different alleles at the (LGS1) locus in these genotypes or recombination between the marker amplified by the primers and the LGS allele. Also genotype Korokollow does not show the typical SRN39 or Shanqui Red marker pattern, but this genotype has a quite atypical strigolactone profile so has perhaps not the standard (LGS1) locus. This suggests involvement of genes other than LGS1 in controlling the low germination traits in some of the genotypes investigated. With the LGS1 gene sequence now available it would be interesting to test its expression in these atypical genotypes. This would help confirm whether the QTL identified for the resistance to Striga, is common in different sorghum populations or is specific for SRN39, which would assist future genetic modification or introgression breeding. If new genes are responsible, this could provide additional sources for the introgression of low germination traits from donors other than SRN39 into new preferred sorghum varieties such as Feteritas using marker-assisted selection (MAS) and backcrossing.

\section{Implications for control methods}

The analysis of strigolactones and their concentration in root exudates of the host and potential trap-, catch-, substitution and intercrops such as groundnut, sesame, millet, cowpea, cotton and soybean could support the right choice of such crops under specific conditions of crop type, Striga ecotype, and climatic conditions. I showed that in intercrops the presence of high amounts of 5-deoxystrigol results in enhanced suicidal germination suggesting that intercropping with cultivars which produce high amounts of such strong stimulants can results in better Striga control. Overexpression of biosynthetic genes responsible for the production of such strong stimulants (e.g. LGS1 from sorghum) may further enhance effective suicidal germination (Sun et al., 2007). Several strigolactones can efficiently induce germination of Striga and possibly also guide chemotropic growth of the Striga radical towards the root of their host (Lendzemo et al., 2007). It would be interesting to confirm such phenomena in the inter-crop species studied. 
In Chapter 4 in pearl millet I showed that the production of high amounts of the less active orobanchol-type strigolactone, orobanchyl acetate, at expenses of strigol-type strigolactones is associated with resistance to the sorghum Striga ecotype. Orobanchyl acetate, however, is highly active in (Striga gesnerioides Vatke.) seed germination, which is not triggered by the synthetic strigolactone GR24. In general, acetates of hydroxystrigolactones are several fold less active than the corresponding free hydroxystrigolactones. It would be interesting to confirm the stimulation activity of orobanchyl acetate in different populations of Striga hermonthica especially ecotypes adapted to pearl millet In addition, mechanisms and conditions underlying strigolactone biosynthesis towards orobanchol-type strigolactones in sorghum, pearl millet and inter-crops merits further investigations. Overall, my results showed the presence of significant associations between type and concentrations of specific strigolactones and root exudate induced Striga germination and Striga parasitism. The negative correlation between strigolactones produced by the intercrops and Striga infection on the intercropped sorghum suggest that suicidal germination is indeed the major cause for the intercrop effect; it will, however, also be exciting to see if different strigolactones will also result in a different microbiome recruitment. Hopefully, the knowledge obtained will help in the selection/breeding or engineering of intercrop cultivars that provide better control of Striga.

\section{Conclusions}

In my thesis I show that the strigolactones play an even bigger role in the control of Striga in cereals than we so far realized. I showed that the amount of 5-deoxystrigol is strongly and directly associated with Striga susceptibility and sensitivity while the amount of orobanchol is strongly and directly associated with resistance and tolerance. This knowledge can provide basic pre-breeding information needed for further improving low stimulant resistance, but on top of that also tolerance. I showed that IS9830, SRN39 and Tetron already combine both resistance and tolerance to Striga, which implies that a combination of both traits is possible. The future challenge is to establish the genetic nature of the resistance and tolerance exhibited by some of the germplasm used in my study using 
available technologies to enable exploitation of both traits. If such traits are, on top of that, also combined with (an) additional resistance mechanism(s) this will provide a better, more appropriate and durable control option against the parasitic witchweed Striga. Finally, also in the use of intercrops strigolactones seem to play a key role. By optimally exploiting this promising control methods through the knowledge obtained in this thesis, in combination with improved host genotypes, a solution for the immense Striga problem may perhaps come within reach.

\section{References}

Akiyama, K., Ogasawara, S., Ito, S. and Hayashi, H. (2010) Structural requirements of strigolactones for hyphal branching in AM fungi. Plant Cell Physiology. 51: 1104-1117.

Akiyama K, Matsuzaki K, Hayashi H (2005) Plant sesquiterpenes induce hyphal branching in arbuscular mycorrhizal fungi. Nature 435: 824-827.

Amusan IO, Rich PJ, Menkir A, Housley T, Ejeta G (2008). Resistance to Striga hermonthica in a maize inbred line derived from Zea diploperennis. New Phytologist 178:157-166.

Ast, A. v., L. Bastiaans and M. J. Kropff, (2000). A comparative study on Striga hermonthica interaction with a sensitive and a tolerant sorghum cultivar. Weed Research 40, 479493.

Atera, E. A., Itoh, K., \& Onyango, J. C. (2011). Evaluation of ecologies and severity of Striga weed on rice in sub-Saharan Africa. Agriculture and Biology Journal of North America, 2(5), 752-760.

Awad, A.A., Sato, D., Kusumoto, D., Kamioka, H., Takeuchi, Y., and Yoneyama, K. (2006). Characterization of strigolactones, germination stimulants for the root parasitic plants Striga and Orobanche, produced by maize, millet and sorghum. Plant Growth Regulation. 48: 221-227.

Babiker, A. G. T. (2007). Striga: The spreading scourge in Africa. Regulation of Plant Growth and Development, (Japan).

Babiker, A.G.T., Ejeta, G., Butler, L.G. and Woodson, W.R., (1993). Ethylene biosynthesis and strigol-induced germination of Striga asiatica. Physiologia Plantarum, 88(2), pp.359-365.

Barker, K. R., 1993. Resistance/tolerance and related concepts/terminology in plant nematology. Plants Disease 77, 111-113.

Bebawi, F. F., \& Eplee, R. E., (1986). Efficacy of ethylene as a germination stimulant of Striga hermonthica seed. Weed Science, 34 (5), 694-698.

Bebawi, F. E, 1981: Intraspecific physiological variants of Striga hermonthica. - Experimental Agricultures. 17: 419-423.

Bebawi, F., and Michael, A., (1991). Bioassay of some economic crops of the Sudan to Striga germination and parasitization, in: 5. International Symposium of Parasitic Weeds, Nairobi (Kenya), 24-30 Jun 1991: CIMMYT.

Bebawi, F.F., R.E. Eplee, C.E. Harris, and R.S. Norris., (1984). Longevity of witchweed (Striga asiatica) seed. Weed Science. 32:494-507.

Botanga, C.J., Alabi, S.O., Echekwu, C.A. and Lagoke, S.T.O., (2003b). Genetics of suicidal germination of Striga hermonthica (Del.) Benth by cotton. Crop science, 43(2), pp.483-488.

Bouwmeester, H.J., Roux, C., Lopez-Raez, J.A., and Becard, G., (2007). Rhizosphere communication of plants, parasitic plants and AM fungi. Trends in plant science 12, (5), 224-230. 
Butler LG (1995) Chemical communication between the parasitic weed Striga and its crop host. A new dimension in allelochemistry. In: Inderjit KM, Dakshini M, Enhelling FA (eds) Allelopathy, organisms, processes and applications. American Chemical Society, Washington, DC, pp 158-166.

Cardoso, C. et al., (2014a). Natural variation of rice strigolactone biosynthesis is associated with the deletion of two MAX1 orthologs. Proceedings of the National Academy of Sciences, U.S.A. 111, 2379-84

Cardoso, C., Charnikhova, T., Jamil, M., Delaux, P. M., Verstappen, F., Amini, M... and Bouwmeester, H. (2014b). Differential activity of Striga hermonthica seed germination stimulants and Gigaspora rosea hyphal branching factors in rice and their contribution to underground communication. PloS one, 9(8), e104201.

Carsky, R. J., L. Singh and R. Ndikawa, 1994b. Suppression of Striga hermonthica on sorghum using a cowpea intercrop. Experimental Agriculture 30, 349-358.

Cechin, I. and M. C. Press, (1993). Nitrogen relations of the sorghum-Striga hermonthica host parasite association: growth and photosynthesis. Plant Cell and Environment 16, 237247.

Chittapur, B. M., Hunshal, C. S., \& Shenoy, H. (2001). Allelopathy in parasitic weed management: role of catch and trap crops. Allelopathy Journal, 8(2), 147-159

Cook, C.E., Whichard, L.P., Turner, B., Wall, M.E., and Egley, G.H. (1966). Germination of witchweed (Striga lutea Lour.): Isolation and properties of a potent stimulant. Science 154: 1189-1190.

Drennan, D. S. H. and S. O. El Hiweris, (1979). Changes in growth regulator substances in Sorghum vulgare infected with Striga hermonthica. In: L. J. Musselman, A. D. Worsham and R. E. Eplee (eds). Proceedings of the Second International Symposium on Parasitic Weeds. North Carolina State University, Raleigh, pp. 144-155.

Ejeta, G. (2007). The Striga scourge in Africa: a growing pandemic. Integrating new technologies for Striga control: towards ending the witch-hunt, Ejeta, G. and Gressel, J., eds. World Scientific Publishing Co. Pte. Ltd., Singapore. pp. 3-16.

Ejeta, G., A. Mohammed, P. Rich, A. Melake Berhan, T. L. Housley and D. E. Hess, (2000). Selection for specific mechanisms for resistance to Striga in sorghum. In: B. I. G. Haussmann, D. E. Hess, M. L. Koyama, L. Grivet, H. F. W. Rattunde and H. H. Geiger, Breeding for Striga resistance in cereals: proceedings of a workshop held at IITA, Ibadan, Nigeria, pp. 29-40

Ejeta, G., Butler, L.G., (1993). Host-parasite interactions throughout the Striga life cycle, and their contributions to Striga resistance. African Crop Science Journal 1.

Ejeta, G., L.G. Butler, and A.G. Babiker. (1992). New approaches to the control of Striga. Research at Purdue University. Agronomy Experimental Station. Bulletin \# 991.

Emechebe, A., Ellis-Jones, J., Schulz, S., Chikoye, D., Douthwaite, B., Kureh, I., et al., (2004). Farmers' perception of the Striga problem and its control in northern Nigeria. Experimental Agriculture. 40(02), 215-232.

Frost, D.L., Gurney, A.L., Press, M.C., and Scholes,J.D.(1997) Striga hermonthica reduces photosynthesis in sorghum: the importance of stomatal limitations and a potential role for ABA? Plant, Cell and Environment 20, $483 \pm 492$

Gbehounou, G. and E. Adango, (2003). Trap crops of Striga hermonthica: in vitro identification and effectiveness in situ. Crop Protection 22, 395-404.

Gebremedhin, W., Goudriaan, J., and Naber, H. (2000). Morphological, phenological and water-use dynamics of sorghum varieties (Sorghum bicolor) under Striga hermonthica infestation. Crop Protection 19(1), 61-68.

Gobena, D., Shimels, M., Rich, P. J., Ruyter-Spira, C., Bouwmeester, H., Kanuganti, S., ... \& Ejeta, G. (2017). Mutation in sorghum LOW GERMINATION STIMULANT 1 alters strigolactones and causes Striga resistance. Proceedings of the National Academy of Sciences, 114(17), 4471-4476. 
Graves, J.D.(1995). Host-plant responses to parasitism. In: Parasitic Plants (Press, M. C. and Graves, J. D., ed.), London: Chapman and Hall, pp.206 \pm 225 .

Gressel J, Hanafi A, Head G, Marasas W, Obilana B, Ochanda J, Souissi T, Tzotzos G (2004) Major heretofore intractable biotic constraints to African food security that may be amenable to novel biotechnological solutions. Crop Protection 23: 661-689

Gressel, J. (2009). Crops with target-site herbicide resistance for Orobanche and Striga control. Pest Management Science, 65(5), 560-565.

Hauck C, Muller S, Schildknecht H (1992). A germination stimulant for parasitic flowering plants from Sorghum bicolor, a genuine host plant. Journal of Plant Physiology

Haussmann BIG, Hess DE, Omanya GO, Reddy BVS, Kayentao M, Welz HG, Geiger HH. 2001. Major and minor genes for stimulation of Striga hermonthica seed germination in sorghum, and interaction with different striga populations. Crop Science 41: 1507-1512.

Haussmann B, Hess DE, Omanya GO, Folkertsma RT, Reddy BVS, Kayentao M, Welz HG, Geiger HH. (2004). Genomic regions influencing resistance to the parasitic weed Striga hermonthica in two recombinant inbred populations of sorghum. Theoretical Applied Genetics 109: 1005-1016.139: 474-478.

Hess, D. E., G. Ejeta and L. G. Butler, (1992). Selecting sorghum genotypes expressing a quantitative biosynthetic trait that confers resistance to Striga. Phytochemistry 31, 493-497.

Hess, D. E. (1989). Resistance to Striga hermonthica (Del.) Benth. in sorghum (Doctoral dissertation, PhD thesis, Purdue University).

Jamil, M., Rodenburg, J., Charnikhova, T., and Bouwmeester, H.J. (2011). Pre-attachment Striga hermonthica resistance of new rice for Africa (NERICA) cultivars based on low strigolactone production. New Phytologist, 192: 964-975

Jamil, M., Charnikhova, T., Verstappen, F., Ali, Z., Wainwright, H. and Bouwmeester, H.J., (2014). Effect of phosphate-based seed priming on strigolactone production and Striga hermonthica infection in cereals. Weed research, 54(3), pp.307-313.

Joel, D. M., (2000). The long-term approach to parasitic weeds control: manipulation of specific developmental mechanisms of the parasite. Crop Protection, 19, 753-758.

Kaewchumnong K, Price AH (2008) A study on the susceptibility of rice cultivars to Striga hermonthica and mapping of Striga tolerance quantitative trait loci in rice. New Phytol 180(1):206-216.

Kambal, A. E., (1979): Striga Research in the Sudan. Technical Progress Report No. 1. Agricutural Research Corporation, Wad Medani and Faculty of Agricuture, Khartoum.

Khan, Z.R., Hassanali, A., Overholt, W., Khamis, T.M., Hooper, A.M., Pickett, J.A., et al., (2002). Control of witchweed Striga hermonthica by intercropping with Desmodium spp., and the mechanism defined as allelopathic. Journal of chemical ecology, 28, (9), 1871-1885.

Khan, Z. R., Pickett, J. A., Hassanali, A., Hooper, A. M., \& Midega, C. A. O. (2008). Desmodium species and associated biochemical traits for controlling Striga species: present and future prospects. Weed Research, 48(4), 302-306.

Kohlen, W., Charnikhova, T., Lammers, M., Pollina, T., Tóth, P., Haider, I., Pozo, M.J., Maagd, R.A., RuyterSpira, C., and Bouwmeester, H.J. (2012). The tomato CAROTENOID CLEAVAGE DIOXYGENASE8 (SlCCD8) regulates rhizosphere signaling, plant architecture and affects reproductive development through strigolactone biosynthesis. New Phytologist 196: 535-547.

Lendzemo, V. W., Kuyper, T. W., Matusova, R., Bouwmeester, H. J., \& Ast, A. V. (2007). Colonization by arbuscular mycorrhizal fungi of sorghum leads to reduced germination and subsequent attachment and emergence of Striga hermonthica. Plant signaling \& behavior, 2(1), 58-62. 
Lopez-Raez, J. A., Charnikhova, T., Gomez-Roldan, V., Matusova, R., Kohlen, W., De Vos, R., and Bouwmeester, H. (2008). Tomato strigolactones are derived from carotenoids and their biosynthesis is promoted by phosphate starvation. New Phytologist, 178(4), 863-874.

Mohemed, N., Charnikhova, T., Bakker, E. J., Ast, A., Babiker, A. G., \& Bouwmeester, H. J. (2016). Evaluation of field resistance to Striga hermonthica (Del.) Benth. in Sorghum bicolor (L.) Moench. The relationship with strigolactones. Pest Management Science, 72 (11), 2082-2090.

Mohamed A, Ellicott A, Housley TL and Ejeta G, Hypersensitive Response to Striga Infection in Sorghum. Crop Science, 43(4): 1320-1324 (2003).

Motonami, N., Ueno, K., Nakashima, H., Nomura, S., Mizutani, M., Takikawa, H. and Sugimoto, Y., (2013a). The bioconversion of 5-deoxystrigol to sorgomol by the sorghum, Sorghum bicolor (L.) Moench. Phytochemistry, 93, pp.41-48.

Musselman, L.J. (1980). The biology of Striga, Orobanche, and other root-parasitic weeds. Annual review of phytopathology, 18(1), 463-489.

Nomura, S., Nakashima, H., Mizutani, M., Takikawa, H., \& Sugimoto, Y. (2013). Structural requirements of strigolactones for germination induction and inhibition of Striga gesnerioides seeds. Plant cell reports, 32(6), 829-838.

Obilana, A.B., Ramaiah, K.V., (1992). Striga (witchweeds) in sorghum and millet: knowledge and future research needs. In: de Milliano, W.A.J., et al. (Eds.), Sorghum and Millet Diseases: A Second World Review. International Crops Research Institute for the Semi-Arid Tropics (ICRISAT), Patancheru, AP 502 324, pp. 187-201.

Omanya, G.O., Haussmann, B.I.G., Hess, D.E., Reddy, B.V.S., Mukuru, S.Z., Welz, H.G., Geiger, H.H., (2000). Evaluation of laboratory, pot, and field measures of striga resistance in sorghum. In: Haussmann, B.I.G., Hess, D.E., Koyama, M.L., Grivet, L., Rattunde, H.F.W., Geiger, H.H. (Eds.), Breeding for Striga Resistance in Cereals. Proceedings of a Workshop, IITA, Ibadan, Nigeria, $18 \pm 20$ August 1999.

Oswald, A. (2005). Striga control-technologies and their dissemination. Crop Protection, 24, 333-342.

Oswald, A., and Ransom, J., (2001). Striga control and improved farm productivity using crop rotation. Crop Protection 20(2), 113-120.

Pageau, K., Simier, P., Le Bizec, B., Robins, R. J., \& Fer, A. (2003). Characterization of nitrogen relationships between Sorghum bicolor and the root-hemiparasitic angiosperm Striga hermonthica (Del.) Benth. using K15NO3 as isotopic tracer. Journal of Experimental Botany, 54(383), 789-799.

Parker C, Riches CR (1993) Parasitic Weeds of the World: Biology and Control. CAB International, Wallingford, UK, pp 111-163.

Press, M.C., Scholes, J.D., and Watling, J.R. (1999). Parasitic plants: physiological and ecological interactions with their hosts. Physiological plant ecology, 175-197.

Ramaiah, K.V. 1987. Breeding cereal grains for resistance to witchweed. p. 227-242. In L.J.Musselman (ed.) Parasitic Weeds in Agriculture, Volume I, Striga. CRC Press, Boca Raton, FL.

Ramaiah, K. V. (1984): Physiological specialization of Striga hermonthica and crop specificity: In Proceedings of the third International Symposium on Farasitic Weeds, Aleppo, 1984. pp 58-65.

Ramlan, M. F., and J. D. Graves (1996). Estimation of the sensitivity to photoinhibition in Striga hermonthicainfected sorghum. Journal of experimental botany. 47.1: 71-78.

Rich, P.J., Grenier, C., and Ejeta, G. (2004). Striga resistance in the wild relatives of sorghum. Crop Science. 44: 2221.

Rodenburg, Jonne, et al. CO2-assimilation and chlorophyll fluorescence as indirect selection criteria for host tolerance against Striga. Euphytica 160.1 (2008): 75-87.

Rodenburg, J., and L. Bastiaans. Host-plant defence against Striga spp.: reconsidering the role of tolerance. Weed Research 51.5 (2011): 438-441. 
Rodenburg, J., Cissoko, M., Kayongo, N., Dieng, I., Bisikwa, J., Irakiza, R., \& Scholes, J. D. (2017). Genetic variation and host-parasite specificity of Striga resistance and tolerance in rice: the need for predictive breeding. New Phytologist, 214(3), 1267-1280.

Sato, D., Awad, A. A., Takeuchi, Y., \& Yoneyama, K. (2005). Confirmation and quantification of strigolactones, germination stimulants for root parasitic plants Striga and Orobanche, produced by cotton. Bioscience, biotechnology, and biochemistry, 69 (1), 98-102.

Schlemper, T. R., Leite, M. F., Lucheta, A. R., Shimels, M., Bouwmeester, H. J., van Veen, J. A., \& Kuramae, E. E. (2017). Rhizobacterial community structure differences among sorghum cultivars in different growth stages and soils. FEMS Microbiology Ecology, 93(8).

Spallek, T., Mutuku, M., and Shirasu, K. (2013). The genus Striga: a witch profile. Molecular plant pathology 14(9), 861-869.

Sugimoto, Y., Wigchert, S. C., Thuring, J. W. J., \& Zwanenburg, B. (1998). Synthesis of all eight stereoisomers of the germination stimulant sorgolactone. The Journal of Organic Chemistry, 63 (4), 1259-1267.

Sun Z, Matusova R, Bouwmeester H (2007) Germination of Striga and chemical signaling involved: A target for control methods In: Integrating new technologies for Striga control. World Scientific Publishing Co. Pte. Ltd., London.

Traore, H., D. Yonli, D. Diallo and P. Sereme, (2011). Suicidal Germination of Striga hermonthica (Del.) Benth. by Cotton, Cowpea and Groundnut Genotypes in Burkina Faso. International Journal of Agricultural Research, 6: 49-57.

Ueno, K., Nomura, S., Muranaka, S., Mizutani, M., Takikawa, H., \& Sugimoto, Y. (2011). Ent-2'-epiorobanchol and its acetate, as germination stimulants for Striga gesnerioides seeds isolated from cowpea and red clover. Journal of agricultural and food chemistry, 59(19), 10485-10490.

Van Ast, A., Bastiaans, L., and Kropff, M.J. (2000). A comparative study on Striga hermonthica interaction with a sensitive and a tolerant sorghum cultivar. Weed Research, 40(6), 479-493.

Vasudeva Rao, M.J., (1985). Techniques for screening sorghums for resistance to Striga. ICRISAT Information Bulletin No. 20. International Crops Research Institute for the Semi-Arid Tropics (ICRISAT), Patancheru PO, Andhra Pradesh 502324, India.

Vogler RK, Ejeta G, Butler LG (1996) Inheritance of low production of Striga germination stimulant in sorghum. Crop Science 36:1185-1191.

Watling, J. R., and M. C. Press. Impacts of infection by parasitic angiosperms on host photosynthesis. Plant Biology 3.03 (2001): 244-250.

Westwood, J. H., Yoder, J. I., Timko, M. P., \& dePamphilis, C. W. (2010). The evolution of parasitism in plants. Trends in Plant Science, 15(4), 227-235.

Xie, X., Kusumoto, D., Takeuchi, Y., Yoneyama, K., Yamada, Y., and Yoneyama, K. (2007). 2'-epi-Orobanchol and solanacol, two unique strigolactones, germination stimulants for root parasitic weeds, produced by tobacco. Journal Agrictural Food Chemistry 55: 8067-8072

Xie, X., Yoneyama, K., Kisugi, T., Uchida, K., Ito, S., Akiyama, K.... \& Yoneyama, K. (2013). Confirming stereochemical structures of strigolactones produced by rice and tobacco. Molecular plant, 6(1), 153163.

Xie, X., Yoneyama, K., \& Yoneyama, K. (2010). The strigolactone story. Annual review of phytopathology, 48.

Xie X, Yoneyama K, Kusumoto D, Yamada Y, Takeuchi Y, Sugimoto Y, Yoneyama K. 2008a. Sorgomol, germination stimulant for root parasitic plants, produced by Sorghum bicolor. Tetrahedron Letters 49: 2066-2068. 
Xie X, Yoneyama K, Kusumoto D, Yamada Y, Yokota T, Takeuchi Y, Yoneyama K. (2008b). Isolation and identification of alectrol as $(+)$-orobanchyl acetate, a novel germination stimulant for root parasitic plants. Phytochemistry 69: 427-431

Yoneyama, K., Awad, A.A., Xie, X., Yoneyama, K. and Takeuchi, Y., 2010. Strigolactones as germination stimulants for root parasitic plants. Plant and Cell Physiology, 51(7), pp.1095-1103.

Yoneyama K, Arakawa R, Ishimoto K, Kim HI, Kisugi T, Xie X, et al., (2015). Difference in Strigasusceptibility is reflected in strigolactone secretion profile, but not in compatibility and host preference in arbuscular mycorrhizal symbiosis in two maize cultivars. New Phytologist, 206, 983-989.

Yoneyama, K., Xie, X., Yoneyama, K. and Takeuchi, Y., (2009). Strigolactones: structures and biological activities. Pest Management Science, 65(5), pp.467-470.

Zwanenburg, B., \& Pospíšil, T. (2013). Structure and activity of strigolactones: new plant hormones with a rich future. Molecular plant, 6(1), 38-62. 


\section{Summary}

Huge yield losses in important staple cereal crops including sorghum (Sorghum bicolor [L.] Moench) (Parker, 2009) are caused by infections by the root parasitic plant Striga hermonthica [Del.] Benth. (Striga) particularly in sub-Saharan Africa. Integrating host genetic resistance and tolerance with agricultural practices that reduce the Striga seed bank in the soil are deemed to be the best control strategy. Striga seeds can remain dormant in the soil for up to 20 years. They will germinate when they perceive specific germination inducing compounds that are secreted from the roots of its host and sometimes non-host into the rhizosphere. It was shown that strigolactones (SLs), are the most potent germination stimulants of Striga seed. Exudation of less active SLs in sorghum was shown to be associated with field resistance to Striga while in pearl millet the production of different SLs seems to make it resistant to the Striga that infects sorghum. In contrast, exudation of high amounts of active SLs by non-host intercrops is expected to results in higher suicidal germination and thus help in Striga control. In this study I used 36 sorghum genotypes, 2 pearl millets cultivars and 4 intercrop cultivars to investigate the role of variation in SL amount and profile in resistance and Striga control through intercropping and to answer the question if they also play a role in tolerance.

In chapter 2 and chapter 3 I focus on studying the role of strigolactones in resistance and tolerance to Striga. Hereto, I used statistical analysis on the combined results of strigolactones profiling, in-vitro germination bioassays, gene expression and molecular marker analysis, crop morphological and physiological traits, and photosynthesis measurements. This showed that the exudation of high amounts of orobachol in combination with low amounts of 5-deoxystrigol and sorgomol by some sorghum genotypes is associated with low root exudate germination stimulatory activity and low Striga infection. Moreover, such genotypes had a higher tolerance to Striga and maintained higher photosynthetic capacity under Striga infection. All this suggests that selection/breeding for high orobanchcol/low 5-deoxystrigol and sorgomol profiles will not only improve pre-attachment resistance but also improve tolerance to the Striga that can 
still attach. In chapter 4 I investigated the role of strigolactones in inter- and rotation crops. I showed that exudation of high amounts of orobanchyl acetate in the root exudate of pearl millet is associated with low germination stimulatory activity and low infection of a Striga sorghum ecotype, making pearl millet a suitable rotation crop. Vice versa exudation of high amounts of 5-deoxystrigol by sesame and groundnuts correlated with high (suicidal) germination stimulatory activity and low Striga infection when sorghum was intercropped with these intercrops. This knowledge enables a more targeted selection/ breeding of resistant rotation crop cultivars or intercrop cultivars that induce more Striga suicidal germination. Knowledge on the sensitivity of the target Striga ecotype for certain strigolactones is important before a specific intercrop can be advised in the process of combating Striga in sorghum and other cereals.

In chapter 5 a field experiment using 5 sorghum genotypes with different strigolactone profiles shows that the results from lab and greenhouse experiments can be translated to the field. For example, sorghum genotypes Fakimustahi and Wadfahel with high production of 5-deoxystrigol and sorgomol and relatively low orobanchol production exhibited high susceptibility to Striga in the field whereas Mogud and Wadbaco with much lower 5-deoxystrigol and sorgomol production and higher orobanchol exhibited field resistance.

In chapter 6 I discuss the main highlights of the present thesis. I showed that there is a relationship between resistance/tolerance and the amount and identity of SL production. I hope that the knowledge generated in my thesis about the role of strigolactones in resistance and tolerance can help breeders with the selection of genotypes better equipped to withstand Striga. In addition I hope that the data generated by me can be used for the identification of the underlying genetic regions which would potentially allow the transfer of resistance and tolerance to elite but susceptible sorghum genotypes. The use of these traits in combination with post-attachment resistance mechanisms should result in a better and durable control of this parasite. 


\section{Acknowledgements}

I would like to express my deepest gratitude to Prof. dr. Harro Bouwmeester, and Prof. dr. Abdel Gabar Eltyb Babiker my project promoters, Harro, my heartfelt thanks for academic guidance, generous supervision and critical remarks on my manuscripts. It was a great luck, honor and pleasure to work with you. You have been always patience, optimistic and gave me high motivations, great support and advices on my research. Thanks Harro for personal support whenever needed and for giving me opportunity to develop such academic expertise. Prof. dr. Abdel Gabar Eltyb Babiker I would like to express my great respect, sincere gratitude and special thanks because without your prompt support and viable help I wouldn't be able to start this project in Netherlands, you are such a kind, patient and considerate parent. You spent a lot of time (even your free hours) on my project when I was in Sudan or in Netherlands, providing a lot of constructive advice and also share correcting my manuscripts which help to complete my thesis and also publish in peer reviewed journals. My sincere thanks also go to my external supervisor Dr. Aad van Ast. Dr. Aad I would like to express my gratitude for your kind advices particularly in the field and greenhouse experiments. I would like to thank Dr. Carolien Ruyter-Spira, Carolien I am thankful for your assistance and steering our group in absence or presence of Harro. Thanks for nice dinners you invited us as a group in your house. I am also thankful to all staff and colleagues of Plant Physiology group for cheerful nice company and social gatherings. Many thanks to Prof. Henk Hilhorst, Dr. Sander van der Krol, Dr. Wilco and Dr. Leonie and Dr. Iris for guidance and scientific discussions. Rina and Margret I offer my sincerest gratitude for your help with administrative issues and I appreciated your warm welcome and social attitude. Many thanks to my former lab colleagues (Dr. Wouter, Jamil, Imran, Peter, Caterina. I am thankful to my colleagues of the parasitic plant team (Yanxia,

Beatriz, Giovanni, Xi, Mahdere, Kristina and Yunmeng) and Plant Physiology lab mates (Johanna, Rik, Cecilia, Deborah, Esmer, Jimmy, Julio, Lemeng, Qing, Jun, Wei, Bing, Bas, Thierry, Leo, Hanzi, Jenwei, Arman, Nafieseh, Mariana, Gonda, Sangseong, Farzaneh, Mark, Desalegn, Phuong, Natalia, Elise, Ralph, Karen and Manus) for supportive attitude 
and amusing conservations. I obliged to Tatsiana, Francel, Diaan, Lidiya, Andrea, Carin, Juriaan, Leo and Marielle for technical support and help with lab work. You're all great and always willing to help whenever needed. I would like to express my sincere thanks to my friends and former colleagues in Sudan University for Science and Technology, Shambat, the Striga Lab (Abdulrahman, Rashida, Amani, Rana, Nahid, Reem, Talal, Ismail, Aisha, Soha, Motasim, Ayman and Ehlam) and my Japanese friends Dr. Samejima and Kowata from (JICA) for their supportive attitude and nice company. I would like to take the chance to show my appreciation to my paranymphs Emilie and Bea for countless help and support during and before my defense. Thanks for your patience and this long waiting for my defense. Thanks to my nice friends, Dr. Felemming and Mundie and their kids Lukas and Nikolas from Denmark and Canada, we have spent pleasant time with all of you, and enjoyed great events. My former Sudanese community in Wageningen (Elsadig abo Yousef and Alia, Mohamed Yagoob, Taj Eddin, Khalda and Yahya) and the recent (Dr. Aziza, Actham, Sana, Dear Fatoma, Sharifa and Abduallah Jasper). You have been great friends, nice brothers and sisters. With you all I enjoyed many nice events. To my family, I express my deepest gratitude. My parents, brothers and sisters you are always have faith and trust on me I am indebted to your understanding, supporting and endless love. Many thanks to my dear brother MohiEddin, you are always supporting our big family. To my brother-in-law Prof. dr. Abdel Wahid Awadalla, I am really indebted and very grateful to your generous life time support, caring and standing behind us at hard times since starting my master degree you have been encouraging and taking care of us over all these years. It is hard to find words to thank you. My dear wife, Raga Awadallah and my children Moneeb and Hatin during these years, you shared with me, ups and downs and happiness, your, constant understanding and support encouraged me to overcome difficulties. I am blessed to have you in my life.

Finally, I would like to sincerely acknowledge financial, and technical support of the funding organizations, I am equally indebted to the Netherlands Organization for International Cooperation in Higher Education (NUFFIC) and the Wageningen University 
sandwich $\mathrm{PhD}$ program for their financial support during the entire period of my project in the Netherlands and in Sudan. I would like also to thank the Japan International Cooperation Agency (JICA), for kind support during execution of the field and lab trials at the College of Agricultural Studies, (SUST) in Sudan.

Nasr, April 2018, Wageningen 


\section{Publication list}

\section{Publications}

1. Nasreldin MA, et al., (2016). Evaluation of field resistance to Striga hermonthica [Del.] Benth. in Sorghum bicolor [L.] Moench. The relationship with strigolactones. Pest Management Science (72):2082-2090

2. Nasreldin MA, et al., (2018). Genetic variation in Sorghum bicolor strigolactones and their role in resistance against Striga hermonthica. Journal of Experimental Botany.

3. Jamil, Muhammad, Tatsiana Charnikhova, Tahira Jamil, Zahid Ali, NasrEldin Mohemed Ahmed, Tom Van Mourik, and Harro J. Bouwmeester. Influence of fertilizer microdosing on strigolactone production and Striga hermonthica parasitism in pearl millet. International Journal of Agriculture \& Biology 16, no. 5 (2014).

4. Nasreldin MA, et al., Genetic variation in tolerance to Striga infection in sorghum: underlying processes and the relationship with strigolactones (in preparation).

5. Nasreldin MA, et al., The role of strigolactones in Striga hermonthica control in sorghum by non-host intercrops (in preparation).

\section{Scientific reports}

1. Nasreldin MA, Maryam, A, Tram, H. Tomas, E and Schots Arjen (2009). Rice based vaccine for malaria (2008). Laboratory of Genetics and Plant Physiology, Wageningen, Wageningen University \& Research Center).

2. Nasreldin MA, Rabosielo K C, and Vos Jan. (2007). Precision Agriculture in Potato Production in Sudan. Wageningen University \& Research, Center for Crop and weed ecology

3. Nasreldin MA, and Van Huis, Arnold (2007). Present Situation and Future Prospects of using Natural Enemies to Control Vegetable Insect pest in Sudan. Laboratory of Entomology, Wageningen University \& Research Center. 
4. Nasreldin MA, R. Navarro, E.E. Wessels \& M. A. Biernat (2008). Identification of unknown baculovirus from tea moth (Buzura suppressaria) from India. Laboratory of Virology, Wageningen University \& Research Center.

5. Nasreldin MA, \& Takken, Willem (2007). Management of insecticide resistance in Mosquito bed nets. Laboratory of Entomology, Wageningen University \& Research Center.

6. Nasreldin MA, Toth Peter and Bouwmeester J Harro (2009).Volatiles organic compounds finger printing in Arabidopsis thaliana in response to O. ramosa and $O$. aegyptiaca infestation. MSc. Thesis Labratory of Plant Physiology, Wageningen University \& Research Center. 


\title{
Education Statement of the Graduate School
}

\section{Experimental Plant Sciences}

\author{
Issued to: Nasreldin Mohemed Ahmed \\ Date: $\quad 6$ April 2018 \\ Group: Laboratorium of Plant Physiology \\ University: Wageningen University \& Research
}

\section{1) Start-up phase}

First presentation of your project

Title: The role of strigolactones in resistances, tolerances and control of Striga in

sorghum

- Writing or rewriting a project proposal

Title: The role of strigolactones in resistances, tolerances and control of Striga in

sorghum

- Writing a review article or book chapter

- MSc courses

MOB-20306 Genomics

2012

PBR-20306 Bio-Informatic technology

2013

- Laboratory use of isotopes

Subtotal Start-up Phase

9.0 credits*

\section{2) Scientific Exposure}

- EPS PhD student days

EPS PhD student day, Utrecht, NL

EPS PhD student day, Wageningen, NL

EPS PhD student day 'Get2Gether', Soest, NL

08 Dec 2011

07 Nov 2012

EPS theme symposia

EPS theme 2 symposium 'Interactions between Plants and Biotic Agents', together with Willie Commelin Scholten Day, Amsterdam, NL

EPS theme 3 symposium 'Metabolism and Adaptation', Wageningen, NL

EPS theme 2 symposium 'Interactions between Plants and Biotic Agents', together with Willie Commelin Scholten Day, Wageningen, NL

$\underline{\text { date }}$

01 Jun 2010

20 May 2011

29-30 Jan 2015

03 Feb 2011

10 Feb 2011

10 Feb 2012

- National meetings (e.g. Lunteren days) and other national platforms

Annual meeting 'Experimental Plant Sciences', Lunteren, NL

Annual meeting 'Experimental Plant Sciences', Lunteren, NL

19-20 Apr 2010

04-05 Apr 2011

- Seminars (series), workshops and symposia

Seminar 'Genome-wide association mapping in Arabidopsis thaliana' (Glenda

Willems)

Symposium 'Improving yield prediction by combining statistics, genetic, physiology and phenotyping: the EU SPICY project', Wageningen, NL

Seminar 'The efficiency of Plant Breeding', Dr. Salvadore (ICARDA)

Seminar Statistics, genetics and omic research (Fred van Eeuwijk, WU)

Symposium 'Increasing homogenity in Global food supplies and implications for food security', Wageningen, NL

Seminar 'Mutualism in Action, Harmony Conflict', Toby Kiers (VU, Amsterdam)

Symposium 'Measuring the Photosynthetic Phenome', Wageningen, NL

24 Aug 2010

07 Mar 2012

16 Sep 2013

08 Dec 2013

11 Mar 2014

'14 Mar 2014

07-09 Jul 2014

\section{- Seminar plus}

- International symposia and congresses

The 11th World Congress on Parasitic Plants, Martina France, Italy

07-12 Jun 2011

The 1st International congress on strigolactones, Wageningen, NL

01-06 Mar 2015 


\section{- Presentations}

Poster: 11th World Congress on Parasitic Plants, Martina France,Italy

07-12 Jun 2011

Poster: 1st International congress on strigolactones, Wageningen, NL

01-06 Mar 2015

Oral: 11th World Congress on Parasitic Plants, Martina France,Italy

07-12 Jun 2011

- IAB interview

- Excursions

ARC Gene Bank, sorghum genotype characterization and selection (Dr EITahir, Sudan)

ARC, Farmer Field School, Sudan (Prof. AGT. Babiker )

Jul 2011

Oct 2012

GeneTwister Company, Wgeningen, NL

\section{3) In-Depth Studies}

\section{- EPS courses or other PhD courses}

R-statistic PhD student workshop, Wageningen, NL

Bioinformatics- A User's Approach, Wageningen, NL

date

- Journal club

Member of the literature disscussion group at Plant Physiology

- Individual research training

\section{Subtotal In-Depth Studies}

5.5 credits*

\section{4) Personal development}

- Skill training courses

PhD Competence Assesment

Techniques for Writing and Presenting a Scientific Paper

Project and Time Management

Information Literacy PhD including Endnote Introduction

Career Perspective

Interpersonal Communication for $\mathrm{PhD}$ Students $\underline{\text { date }}$

18 May 2010

06-09 Sep 2011

Sep-Nov 2011

25-26 Feb 2014

18, 25 Sep-02, 09, 16 Oct 2014

05-06 Oct 2011

01-06 Mar 2015

1st International strigolactone conference, Wageningn university,NL

Membership of Board, Committee or PhD council

Subtotal Personal Development

7.3 credits*

\section{TOTAL NUMBER OF CREDIT POINTS*}

33.5

Herewith the Graduate School declares that the PhD candidate has complied with the educational requirements set by the Educational Committee of EPS which comprises of a minimum total of 30 ECTS credits

${ }^{*} A$ credit represents a normative study load of 28 hours of study. 